Journal of the Scientific Agricultural Society of Finland

Vol. 49: 1-106, 1977

Maataloustieteellinen Aikakauskírja

\title{
DER EINFLUSS VON VERRINGERTEN NITRIT- UND NITRATZUSÄTZEN AUF DIE EIGENSCHAFTEN DER ROHWURST
}

Abstract: The effect of lowered addition of nitrite and nitrate on the properties of dry sausage

Selostus: Alennettujen nitriitti- ja nitraattilisäyksien vaikutus kestomakkaran ominaisuuksiin

E E R O P U O L N N E

Institut für Fleischtechnologie, Universität Helsinki

Viikki, 00710 Helsinki 71, Finnland

WIRD MIT GENEHMIGUNG DER LAND- UND FORSTWIRTSCHAFTLICHEN FAKULTÄT DER UNIVERSITÄT Helsinki aM 25 MÄrz UM 12 Uhr im AUditoriUm XII ZUR ÖFFENTLICHEN VERTEIDIGUNG VORGELEGT. 


\section{Vorwort}

Die vorliegende Untersuchung wurde im Institut für Fleischtechnologie der Universität Helsinki durchgeführt und überwiegend aus Mitteln der Akademie von Finnland finanziert. Bei der Durchführung der Untersuchung fand ich von verschiedener Seite Unterstützung.

Mein ganz besonderer Dank in dieser Beziehung gilt meinem Vorgesetzten, Herrn Prof. Dr. F. P. Niinivaara, der mich während meiner Arbeit unermüdlich anspornte und mit neuen Ideen in grossartiger Weise zum Gelingen derselben beitrug.

Die Herren Professoren Dr. Esko Nurmi sowie Dr. L. Leistner und ihre Mitarbeiter leisteten mit ihren Ratschlägen wertvolle Hilfe, für die ich ihnen an dieser Stelle herzlich danke.

$\mathrm{Zu}$ grossem Dank verpflichtet bin ich auch meiner langjährigen Mitarbeiterin, cand. d. Land- u. Forstwirtschaft Paula Törmä, die mir den Grossteil der technischen Arbeiten abnahm und die Versuchsergebnisse mit grösster Sorgfalt in Diagrammform brachte, cand. d. Land- u. Forstwirtschaft Marita Liljemark, die die Bestimmungen durchführte und in mannigfaltiger Weise beim Abfassen der Arbeit mithalf, und cand. d. Land- u. Forstwirtschaft Riitta Saarnio, welche die Stickoxidmyoglobingehalts-Bestimmungen ausführte.

Bei Lic. med. vet. Eija Seuna und Fleischermeister Pauli Hill bedanke ich mich für die Durchführung der Salmonellenversuche.

Cand. hum. Sirkka Viiliäinen leistete mir unschätzbare Hilfe indem sie die umfangreichen Schreibarbeiten übernahm und mit grösster Sorgfalt und Geduld ausführte. Die Úbersetzung ins Deutsche besorgte Dipl.-Ing. H. Hemmann. Ihnen beiden meinen besten Dank.

Die in der Untersuchung verwendeten Bakterienkulturen stellte die Firma Rudolf Müller \& Co zur Verfügung, für deren wohlwollendes Verhalten gegenüber meiner Arbeit ich sehr dankbar bin.

Weiter möchte ich den zahlreichen in- und ausländischen Freunden und Kollegen danken, die mich mit ihren Ratschlägen in selbstloser Weise unterstützten.

Zum Schluss erlaube ich mir, der Agrikulturwissenschaftlichen Gesellschaft in Finnland zu danken, die diese Arbeit in ihre Publikationsreihe aufgenommen hat.

Helsinki, im Dezember 1976

Eero Puolanne

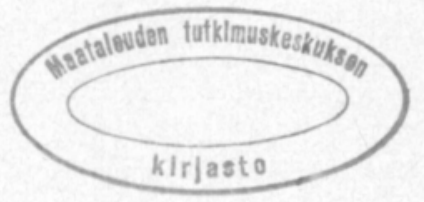




\section{INHALTSVERZEICHNIS}

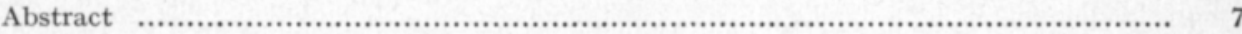

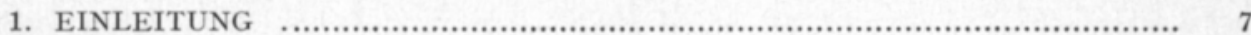

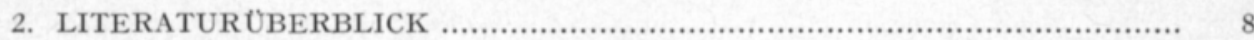

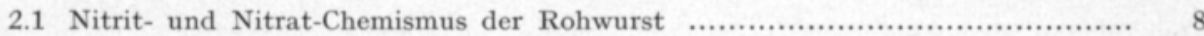

2.2 Technologie des Nitrit- und Nitrateinsatzes bei Rohwurst ......................... 16

2.3 Pathogene Bakterien in der Rohwurst ......................................... 20

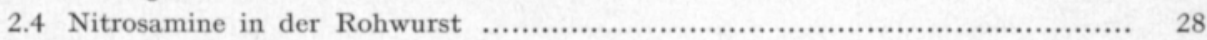

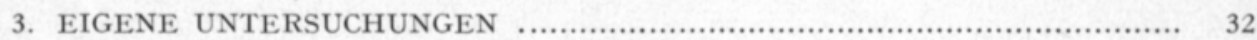

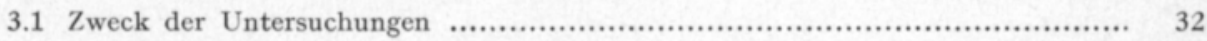

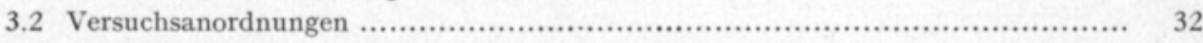

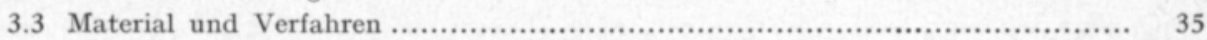

3.3.1 Rohstoffe und Herstellung der Versuchswürste ........................... 35

3.3.2 Chemische Verfahren ............................................................ 36

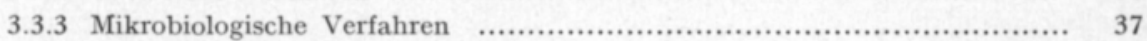

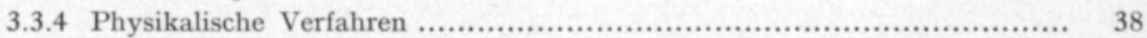

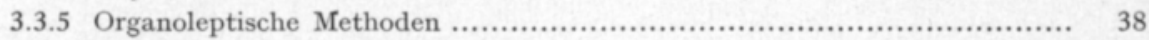

3.3.6 Verarbeitung der Ergebnisse ............................................... 39

3.4 Ergebnisse der Präliminarversuche ............................................. 39

3.4.1 Nitrit- und Nitratzusatz ................................................... 40

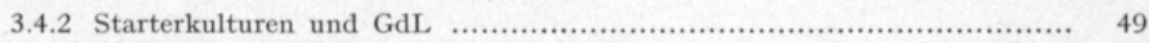

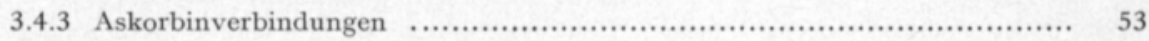

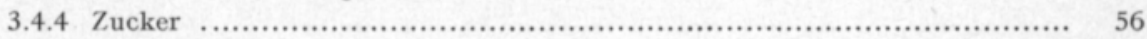

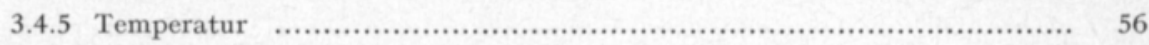

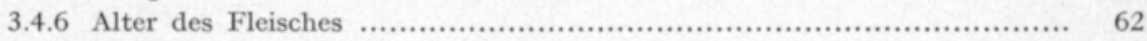

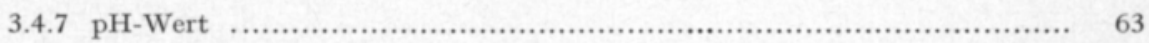

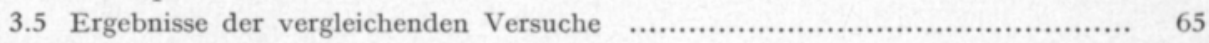

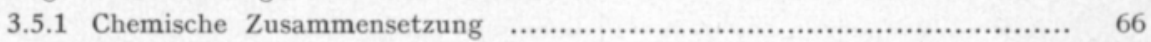

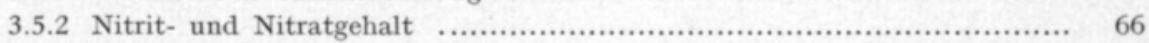

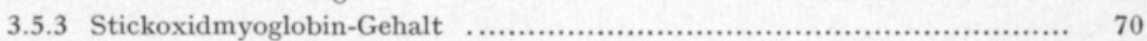

3.5.4 Mikrobiologische Ergebnisse ..................................................... 70

3.5.5 pH-Wert, $a_{w}$-Wert, Konsistenz und Gewichtsverlust ...................... 72

3.5.6 Farbe ............................................................................. 76

3.5.7 Geruchs-, Geschmacks- und Gesamtpunkte ................................. 78

3.5.8 Die zwischen Nitrit- und Nitratmenge sowie verschiedenen Eigenschaften der Rohwurst festgestellten Korrelationskoeffizienten .................... 78

3.6 Einfluss von Nitrit und Nitrat auf das Wachstum von Salmonellen ................ 79

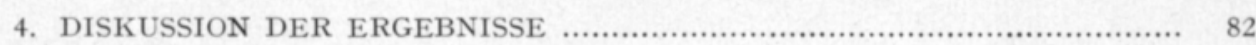

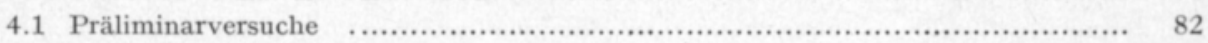

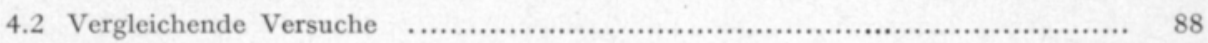

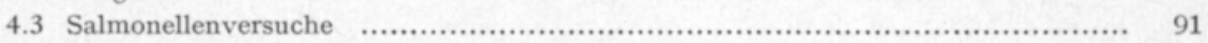

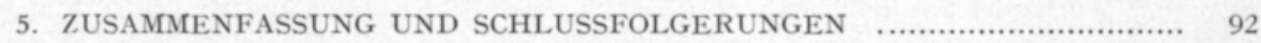

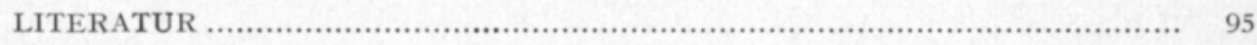

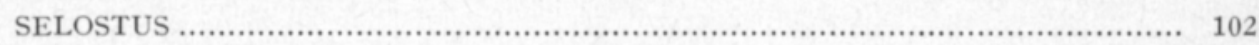

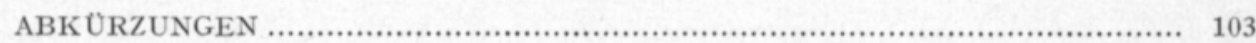

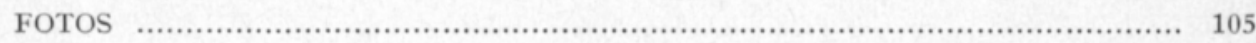





\title{
Puolanne, E. 1977. The effect of lowered addition of nitrite and nitrate on
} the properties of dry sausage. J. Scient. Agric. Soc. Finl. 49: 1-103.

\begin{abstract}
In this study the effects of adding lowered amounts of nitrite and nitrate on the properties and ripening of dry sausage were investigated. This was done in order to ascertain the minimum amounts of additives necessary for the production of sausage that is safe with regard to both technology and pathogenic bacteria. The following analyses were carried out on the sausages, depending on the sample: chemical composition, nitrite and nitrate content, total bacterial count, lactobacilli, micrococci, faecal streptococci, coliform bacteria and yeasts, and $\mathrm{pH}, \mathrm{a}_{\mathrm{w}}$, consistency and weight loss. The sausages were prepared with starter cultures (Duploferment 66) or with glucono-deltalactone $(\mathrm{GdL})$. A total of 208 series of sausage were prepared.

Preliminary experiments showed that a uniform lasting colour was obtained using $25 \mathrm{ppm} \mathrm{NaNO}_{2}$ or $50 \mathrm{ppm} \mathrm{KNO}_{3}$. However, the addition of less than $100 \mathrm{ppm}$ nitrite or $125 \mathrm{ppm}$ nitrate caused the $\mathrm{pH}$ to drop to too low a level because of overly rapid growth of bacteria. Dropping the ripening temperature from $26^{\circ} \mathrm{C}$ to $18^{\circ} \mathrm{C}$ caused slower reduction of nitrate and a higher final $\mathrm{pH}$. Using $\mathrm{GdL}$ and lowering the addition of nitrite from $200 \mathrm{ppm}$ to $100 \mathrm{ppm}$, there was no statistically significant effect on the aroma, taste, or surface colour, physical or microbiological properties or on the growth of Salmonella senftenberg. Neither when using starter cultures were these properties influenced by lowering the addition of nitrate from $300 \mathrm{ppm}$ to $150 \mathrm{ppm}$ or replacing the nitrate with $50 \mathrm{ppm} \mathrm{NaNO}_{2}$ and $75 \mathrm{ppm} \mathrm{KNO}_{3}$. The residual nitrite content was accounted for by $10-15 \mathrm{ppm} \mathrm{NaNO}_{2}$; the residual nitrate content, however, was three to five times greater (c. $100 \mathrm{ppm} \mathrm{KNO}_{3}$ ) in $\mathrm{GdL}$ sausages than in the sausages containing starter culture $\left(20-28 \mathrm{ppm} \mathrm{KNO}_{3}\right)$. In all of the experiments the residual nitrite content did not decrease further once the $\mathrm{pH}$ had reached 5,0. This turning point in nitrate reduction came at $\mathrm{pH} 5,2-5,3$.

Based on this research and on a study of the related literature, we can say that from the point of view of technology and health, safe dry sausage can be prepared using $100 \mathrm{ppm} \mathrm{NaNO}_{2}$ or $150 \mathrm{ppm} \mathrm{KNO}_{3}$, or a mixture of $50 \mathrm{ppm} \mathrm{NaNO}$ and 75 ppnı $\mathrm{KNO}_{3}$. A condition for lowering the amounts of nitrite or nitrate is, however, that GdL and/or starter culture be used at the same time. Using these ensures a sufficiently rapid drop in $\mathrm{pH}$ to the desired value $(\mathrm{pH} 5,3-4,8)$.
\end{abstract}

\section{Einleitung}

Nitrat und Nitrit werden bei Fleischerzeugnissen seit langem zur Verbesserung der Farbbeständigkeit und Haltbarkeit verwendet.

Die akute toxische Wirkung des Nitrits ist auch seit langem bekannt; es reagiert mit Hämoglobin und behindert so die Versorgung der Muskeln mit 
Sauerstoff. In Tierversuchen hat sich eine Dosis von etwa $300 \mathrm{mg} / \mathrm{kg}$ als tödlich erwiesen (Review: Tannenbaum 1976). Für den Menschen liegt die letale Dosis wesentlich niedriger. Die giftigen Wirkungen des Nitrats treten im wesentlichen erst nach seiner Reduktion zu Nitrit auf (FAO/WHO 1974).

Gemüse enthält so hohe Mengen Nitrat, dass, kommt es zur Reduktion, die Gefahr einer Vergiftung gegeben ist, wie man sie bei Säuglingen auch bereits beobachtet hat (Review: Tannenbaum 1976). Das Risiko einer akuten Vergiftung durch Fleischwaren besteht nur im Falle von Herstellungsfehlern, etwa wenn die gesamte Salzmenge versehentlich in Form von Nitrit zugesetzt wird, wodurch Farbe, Geschmack und Konsistenz der Ware so verändert werden, dass diese nicht mehr für den menschlichen Genuss taugt.

Später stellte man fest, dass es bei der Umsetzung zwischen Nitrit und sekundären Aminen zur Bildung von teilweise stark karzinogenen Nitrosaminen kommt (Magee und Barnes 1967, Lijinsky und Epstein 1970). Unter den in Fleischwaren vorliegenden Bedingungen kann es beim Arbeiten mit üblichen Konzentrationen bereits während der Herstellung und Lagerung zur Bildung geringer Nitrosaminmengen kommen.

Ausserdem kann das Restnitrit oder nach der Reduktion das Restnitrat auch erst im Magen mit Aminen reagieren (SANDER und SEIF 1969). Nachdem Nitrosamin-Synthese und Karzinogenität von N-Nitrosoverbindungen durch Tierversuche einwandfrei aufgezeigt worden waren, war eine Neueinschätzung des Nitrit- und Nitratzusatzes in Fleischwaren fällig. Der Beweis, dass die in Fleischwaren enthaltenen Nitrosaminmengen beim Menschen Krebs verursachen, konnte freilich bisher nicht erbracht werden; ausserdem ist Nitrit bzw. Nitrat bei der Herstellung heutiger Produkte unerlässlich. Ein Weglassen dieses Stoffes bzw. dieser Stoffe würde ein erhöhtes gesundheitliches Risiko durch pathogene Bakterien und ein schnelleres mikrobiologisches Verderben der Ware zur Folge haben.

Die FAO/WHO (1974) empfiehlt als maximal zulässige Tagesdosis 0,2 $\mathrm{mg} / \mathrm{kg} \mathrm{NaNO} \mathrm{Nan}_{2}$ und $5 \mathrm{mg} / \mathrm{kg} \mathrm{KNO}_{3}$. Das im Speichel vorhandene, aus Nitrat reduzierte Nitrit führt ausserdem zu einer kurzzeitigen Erhöhung der Nitritkonzentration im Magen. Nach heutiger Erkenntnis können auch die in Fleischwaren enthaltenen Nitrit-Derivate an der Synthese von N-Nitrosoverbindungen teilnehmen, so dass die in der Analyse als sog. "freies Nitrit" ausgewiesene Menge lediglich ein Teilbild von der tatsächlichen Krebsgefahr liefert (Proc. ISNMP 2. 1977). Es besteht somit aller Grund, eine Verringerung der Nitritund Nitratzusätze bei Fleischwaren anzustreben.

\section{Literaturüberblick}

\section{1 Nitrit- und Nitrat-Chemismus in der Rohwurst}

Nitrit ist die wichtigste chemische Verbindung im Hinblick auf Farbe, Haltbarkeit und zahlreiche andere technologische Eigenschaften der Rohwurst. Nitrat wird erst nach seiner Reduktion zu Nitrit wirksam. Die Nitritumsetzung ist in der anorganischen und organischen Chemie viel untersucht 
worden, u.a. auch im Hinblick auf die Belange der Fleischındustrie, wobei sowohl Modell- als auch Objektversuche durchgeführt wurden. Infolge des komplexen Charakters des Fleisches und des Vermögens der den Fleischwaren zugesetzten, relativ geringen Nitritmenge, mit sehr vielen verschiedenen Bestandteilen des Fleisches zu reagieren, kennen wir heute die chemischen, mikrobiologischen und toxikologischen Eigenschaften des Nitrits im Fleisch lediglich in ihren Hauptzügen.

Je nach Tierart, -alter und verschiedenen anderen Faktoren enthält Muskelfleisch 2-20 g/kg $(0,1-1,1 \mathrm{mM}$ ) Myoglobin (GöTse 1969, Bodwell und McClaIN 1971) und auch erhebliche Mengen an Hämoglobin (Review: Fox 1966). Bei der Wurstherstellung tritt das Myoglobin nicht nur als solches (Purpur), sondern auch als Oximyoglobin (Hellrot) und Metmyoglobin (Braungrau) auf. Infolge Sauerstoffeinwirkung (BRooks 1938), der oxydierenden Wirkung des Nitrits (Fox 1966) und der Autoxydation (SNYDER und AYRES 1961) werden Myoglobin und Oximyoglobin in Metmyoglobin umgewandelt. Gleichzeitig reagiert das Nitrit nach seiner Disproportionierung zu Stickstofftrioxid mit den Reduktionsmitteln, wobei es zur Bildung eines Komplexes kommt, der zu schnellem Zerfall in seine Ausgangsstoffe oder teilweise in Reduktionsmittelradikal und Stickoxid neigt. Das Stickoxid verbindet sich mit Metmyoglobin zu Stickoxidmetmyoglobin (Graurot), das leicht zu Stickoxidmyoglobin (helles Hellrot) reduziert wird (Fox und THомson 1963).

Fox und Thomson (1963) benutzten in ihren Versuchen als Reduktionsmittel Askorbinsäure, jedoch lässt sich die entsprechende Reaktion auch mit anderen Reduktionsmitteln erzielen (Fox und Ackerman 1968, Mirna und Hofmann 1969, Fox und Nicholas 1974). Im Muskelfleisch sind insgesamt etwa 50-100 mM titrierbare reduzierend wirkende Verbindungen (REGIER und TAPPEL 1956), darunter ca. $20 \mathrm{ppm}$ endogene Askorbinsäure (NirnivaARA und Antila 1972) enthalten. Der Rohwurst werden 100-500 ppm Askorbinverbindungen zugesetzt (WIRTH 1974). Auch Thiole spielen offensichtlich bei der Umrötung und dem Nitritabbau eine wesentliche Rolle (Mrrna und HoFMann 1969, Dilova u.a. 1976), jedoch verläuft nach Fox und Nicholas (1974) die Umsetzung langsamer als mit Askorbinverbindungen, und ausserdem ist im Fleisch gegenüber Nitrit und Myoglobin ein Uberschuss $(21-25 \mathrm{mM})$ an freien SH-Gruppen vorhanden (Mirna und Hofmann 1969), ohne dass es jedoch zu einem vollständigen Nitritabbau oder einer vollständigen Stickoxidmyoglobin-Bildung kommt.

Die Stickoxidmyoglobin-Bildung wurde sowohl als nichtenzymatischer (KoIzumi und Brown 1971) als auch als enzymatischer (WALters u.a. 1968) Vorgang interpretiert. Diese Modelle und die Reaktionshypothese von Fox und Tномson (1963) ergänzen einander, wobei die entsprechenden Umsetzungen im Fleisch vielleicht auf verschiedene Weise erfolgen (MöHLER 1974).

Zur vollständigen Umsetzung des Fleisch-Myoglobins in Stickoxidmyoglobin sind etwa 8-80 ppm $(0,1-1,1 \mathrm{mM})$ Nitrit erforderlich. Im allgemeinen werden $60-90 \%$ des Gesamtmyoglobins in Stickoxidmyoglobin umgewandelt (MöHLER 1959, Mrrna 1973). Der Anteil des Muskelfleisches in der Wurst ist im allgemeinen zu gering um die oben angegebene Myoglobinkonzentration von $20 \mathrm{~g} / \mathrm{kg} \mathrm{zu}$ erreichen. IngRam (1974) konstatiert, dass bei Fleischwaren eine 
beständige Farbe sogar durch Zusatz von nur 20 ppm $\mathrm{NaNO}_{2}$ erzielt wird. Nach Cassens u.a. (1976) werden für die Stickoxidmyoglobin-Bildung 7,522,5 ppm (5-15\% der zugesetzten Menge) Nitrit verbraucht, wobei diese Menge jedoch von Fleischerzeugnis zu Fleischerzeugnis stark variiert.

Fox und Thomson (1963) haben gründliche Untersuchungen über den Umrötungsmechanismus sowie den Einfluss verschiedener Konzentrationsfaktoren und des $\mathrm{pH}$-Wertes auf die Stickoxidmyoglobin-Bildung bei $20^{\circ} \mathrm{C}$ angestellt. Hierbei ist jedoch zu bedenken, dass diese viel zitierten und erörterten Ergebnisse die Reaktionsgeschwindigkeit in Lösungen angeben, so dass zumindest was die Rohwurst anbelangt bei der Einschätzung der definitiven Umrötung auch der Zeitfaktor in Rechnung zu stellen ist.

Nach Fox und Thомson (1963) war die Stickoxidmyoglobin-Bildungsgeschwindigkeit bis zu einem Nitrit-Myoglobin-Molverhältnis von 5:1 direkt proportional zur Nitritkonzentration; danach wurde keine nennenswerte $\mathrm{Zu}$ nahme der Reaktionsgeschwindigkeit mehr beobachtet. REITH und Szakály (1967 a und b) erzielten die optimale Umrötungsgeschwindigkeit in Lösungen bei einem Molverhältnis von $1: 1$, in Fleisch bei $5: 1$. Hohe Nitritkonzentrationen haben andererseits die Oxydation des Myoglobins zu Cholemyoglobin ("nitrite burn») oder die Bildung von Metmyoglobinnitrit (Braunrot) zur Folge (Fox und Thomson 1964).

Die Stickoxidmyoglobin-Bildungsgeschwindigkeit nahm direkt proportional zur Quadratwurzel des Askorbat-Metmyoglobin-Molverhältnisses zu. Zu hohe Askorbinsäurekonzentrationen $(15 \mathrm{mM})$ verursachen allerdings Gasbildung (NO) und grüne Pigmente. Der $\mathrm{pH}$-Wert spielte jedoch die entscheidende Rolle in bezug auf die Stickoxidmyoglobin-Bildungsgeschwindigkeit (Bild 1). Nach Bild 1 verläuft die Stickoxidmyoglobin-Bildung bei $\mathrm{pH} 6-7$ nur sehr zögernd; unterhalb von $\mathrm{pH} 6$ nimmt jedoch die Reaktionsgeschwindigkeit rasch zu. Unterhalb von $\mathrm{pH} 4,5$ ist eine schnelle Stickoxidmyochromogen-Bildung aus Farbstoff zu verzeichnen, und unterhalb von $\mathrm{pH} 4,0$ entstehen grüne Verbindungen (Fox und Thomson 1963). Nach Reith und SzakÁly (1967 a)

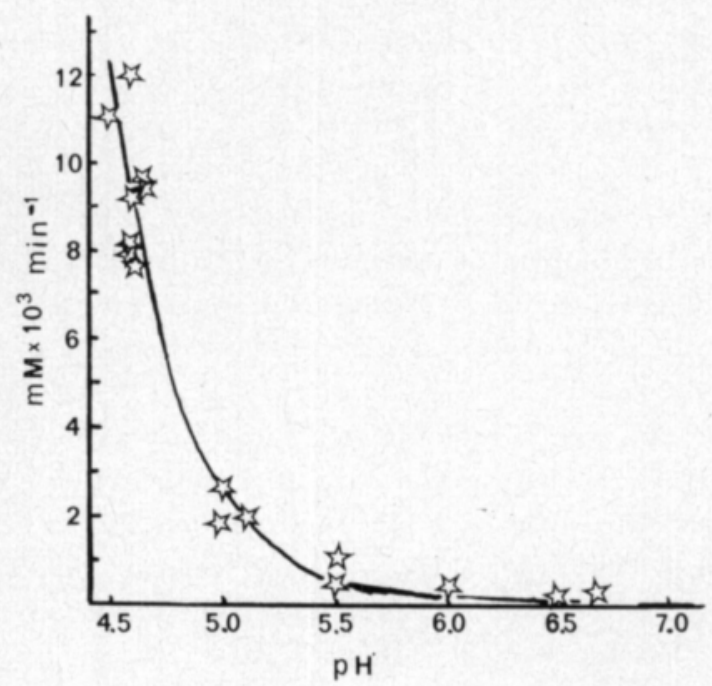

Bild 1. Geschwindigkeitskonstante 0 . Ordnung der Stickoxidmyoglobin-Bildung in Abhängigkeit vom $\mathrm{pH}$-Wert (Fox und Thomson 1963). 
war die Farbe des entsprechenden Stickoxidmyoglobins nicht vom pH-Wert abhängig, wenngleich die Reaktionsgeschwindigkeit mit Sinken des pH-Werts zunahm.

Nach Fox und Thomson (1963) ist oberhalb von pH 5,4 möglicherweise die Metmyoglobin-Reduktion die die Umrötungsgeschwindigkeit begrenzende Reaktion, unterhalb dieses $\mathrm{pH}$-Wertes jedoch der Zerfall des vom Reduktionsmittel und Stickoxid gebildeten Komplexes. Nach MöHLer (1958) nimmt mit sinkendem $\mathrm{pH}$ die sich bildende Stickoxidmyoglobin-Menge relativ zu und erreicht ihr Maximum bei 5,0. Auch nach Brooks (1937) nahm die Geschwindigkeit, mit der sich das Stickoxid ans Hämoglobin bindet, mit abnehmendem $\mathrm{pH}$ in der Form zu, dass die Halbwertszeit bei $\mathrm{pH}$ 5,66 $10 \mathrm{~min}$, bei $\mathrm{pH} \mathrm{5,38} 4 \mathrm{~min}$ und bei $\mathrm{pH} 5,151 \mathrm{~min}$ betrug. Fox (1966) konstatierte, dass Myoglobin und Hämoglobin gewöhnlich in etwa gleicher Weise reagieren, und dass lediglich die Reaktionsgeschwindigkeiten der beiden Stoffe variieren. Bei Rohwurst erfolgt die Reduktion von Nitrat im $\mathrm{pH}$-Bereich 6,0-5,6 (5,4), die Umrötung im Bereich 5,4-5,0 (NinnivaARA 1955).

Die Bildung von Stickoxidmyoglobin erfolgt stets über die Zwischenstufe Metmyoglobin (MöHLER 1970). Das Einfrieren von Fleisch, niedriger Sauerstoffpartialdruck, niedriger $\mathrm{pH}$, hohe Temperatur (HAm und Schweiger 1964) sowie Zusatz von Nitrit und Kochsalz (MöHLer 1970) bewirken Metmyoglobin-Bildung, was an sich im Hinblick auf die Stickoxidmyoglobin-Bildung nicht von Nachteil ist; jedoch haben diese gleichen Faktoren auch die Denaturierung von Metmyoglobin zu Metmyochromogen (Grau) zur Folge (Review: LAWRIE 1974), welches nur noch in geringem Masse roten Farbstoff bildet (REITH und SzakÁly 1967 a). Ausserdem müssen aber im Fleisch auch solche Verhältnisse vorliegen, dass eine Rückreduktion von Stickoxidmetmyoglobin in Stickoxidmyoglobin erfolgt, was im allgemeinen der Fall ist (Fox und Thomson 1963, Walters u.a. 1968, Kolzumi und Brown 1971).

Nitrit wird nicht nur für die Stickoxidmyoglobin-Bildung, sondern auch im Zusammenhang mit anderen Umsetzungen verbraucht. Nitrit bewirkt die Oxydation von Oxihämoglobin zu Methämoglobin und wird dabei selbst zu Nitrat oxydiert, das in einer zum gebildeten Methämoglobin äquimolaren Menge anfällt (MöHLER 1970) (Gleichung 1):

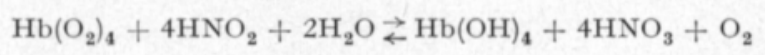

Das Myoglobin reagiert offensichtlich in nahezu gleicher Weise (MöHLER 1970). Auch im Zusammenhang mit anderen Umsetzungen bildet sich aus Nitrit Nitrat (Review: ZATó̆IL und BRÜN 1963).

Nitrit reagiert mit SH-Gruppen der Proteine unter Bildung von Nitrosothiolen oder Disulfiden, mit Aminoverbindungen unter Bildung von freiem Stickstoff oder N-Nitrosaminen. Auch durch die Umsetzungen mit Askorbinsäure, Amiden, Aminen, ungesättigten Fettsäuren, Schwefelwasserstoff u. dgl. reaktionsfähigen Verbindungen sowie mit anorganischen Stickstoffverbindungen kommt es zu einer Nitritabnahme (Review: ZatočIL und BRÜn 1963, Mirna und Hofmann 1969, Cassens u.a. 1976). Allerdings weiss man noch nicht genau genug, in welchem Grade dieses umgesetzte Nitrit in Fleisch- 
waren eine positive (Umrötung, Mikrobenhemmung, Geschmack, Aroma) oder eine negative, d.h. toxische Wirkung ausübt. Die nach dem Herstellungsprozess noch vorhandene Menge an freiem Nitrit ist freilich, verglichen an der zugesetzten Nitritmenge, nur noch von nachgeordneter Bedeutung (Proc. ISNMP 2. 1977).

Nitrit wird im Fleisch gewöhnlich erst in Form von salpetriger Säure wirksam. Die verfügbare Menge an salpetriger Säure ist gemäss Gleichung (2) durch den $\mathrm{pH}-$ Wert festgelegt:

$$
\begin{aligned}
\log _{10} \frac{\left[\mathrm{HNO}_{2}\right]}{\left[-\mathrm{NO}_{2}\right]}=\mathrm{pK}_{\mathrm{a}}-\mathrm{pH} \\
\mathrm{pK}_{\mathrm{a}}=\text { Dissoziationskonstante d. Säure } \\
=3,4
\end{aligned}
$$

Da es sich aber hier um eine normale Gleichgewichtsreaktion handelt, bleibt die relative Menge an salpetriger Säure in Abhängigkeit vom pH-Wert konstant, selbst dann, wenn bei der Gesamtmenge aus diesen oder jenen Gründen Verluste eintreten. Mit zunehmender Temperatur steigt allerdings der $\mathrm{pK}_{\mathrm{a}}$-Wert rasch an (AnON. 1974).

Nach Mirna (1971) kommt es bei Absinken des pH-Werts unter 5,8 zur Bindung von Stickoxid an die Fleischproteine, und im $\mathrm{pH}$-Bereich $\mathbf{5 , 0 - 5 , 8}$ ist ein wesentlicher Teil des Stickoxids auf diese Weise gebunden. OLsman (1977 b) hat beobachtet, dass in erhitztem Fleisch auch die an Proteine gebundene Nitritmenge mit abnehmendem $\mathrm{pH}$ steigt. Desgleichen konstatierte er, dass ein Teil des Nitrits labil zu einer nitrosierenden Verbindung ( $\mathrm{X}-\mathrm{NO})$ gebunden wird, die später als Nitritspeicher fungiert (Olsman 1977 a). ANdo u.a. (1971) stellten ferner einen Einfluss der niedermolekularen Verbindungen des Fleisches auf die Nitritabnahme fest.

Nach NoRdin (1969) erfolgte die Nitritabnahme im Fleisch nach folgender Gleichung (3):

$$
\log _{10}(1 / 2-\text { Zeit })=0,65-0,025 \mathrm{~T}\left({ }^{\circ} \mathrm{C}\right)+0,35 \mathrm{pH}
$$

Allerdings blieb der pH-Bereich unter 5,6 ausserhalb seiner Untersuchung, und die Versuchstemperaturen waren im Hinblick auf wärmebehandelte Fleischerzeugnisse gewählt worden. Nach Olsman und KroL (1972) folgte die Nitritabnahme bei $\mathrm{pH}$ 6,0 und darüber der Kinetik einer Reaktion 1. Ordnung, unter $\mathrm{pH}$ 6,0 der Kinetik einer Reaktion 1. oder 2. Ordnung.

Sie stellten auch fest, dass die Nitritabnahme mit wachsendem FleischWasser-Verhältnis an Geschwindigkeit verliert. Dies könnte von Bedeutung in bezug auf den Restnitritgehalt getrockneter Fleischwaren sein (HiLl u.a. 1973). Nach Fox und Nicholas (1974) bestand zwischen Nitritabnahme und Stickoxidbildung eine Beziehung. Woolford u.a. (1976) betonen die Rolle, die die Proteine bei der Nitritabnahme spielen, denn sie vermochten $60 \%$ des "verschwundenen" Nitrits ( $\mathrm{pH} 5,5, \mathrm{~T}+20^{\circ} \mathrm{C}$ ) unter Anwendung der ${ }^{15} \mathrm{~N}$-Technik in den Proteinen nachzuweisen. CAssens u.a. (1977) haben eine Zusammenfassung der von mehreren Forscher-Teams unter Anwendung von Isotopentechnik ermittelten Ergebnisse über die Nitritverteilung ausgearbeitet (Tabelle 1), in der sie konstatieren, dass sich nur $\mathbf{1 - 5} \%$ des Nitrits 


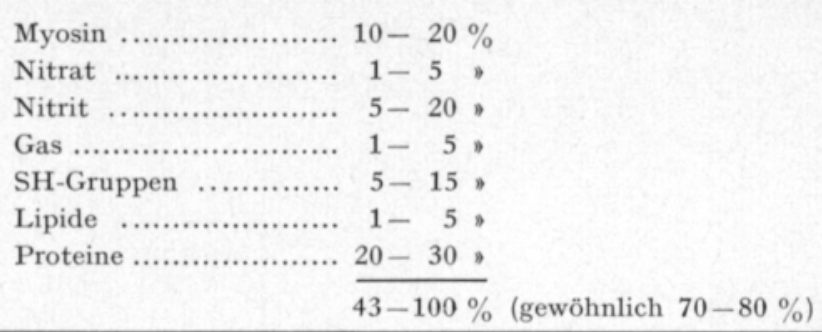

verflüchtet haben während der restliche Teil in einer Form vorliegt, die ein potentielles toxikologisches Risiko in sich schliesst.

Die Stabilisierung der Fleischfarbe erfolgt durch die Denaturierung von Stickoxidmyoglobin zu Stickoxidmyochromogen (Hellrot). Herbeigeführt wird diese Reaktion durch Erhitzen und $\mathrm{pH}$-Wert-Senkung (Fox und Thomson 1963, Reith und SzakÁly 1967 b). Die pH-Abnahme in der Rohwurst ist somit ein farbstabilisierender Faktor (NinnivaARA 1955). Tarlagdis (1962) hat eine Hypothese aufgestellt, der zufolge beim Stickoxidmyochromogen der Globinteil losgelöst ist und ans Häm zwei NO-Moleküle angelagert sind. Cassens u.a. (1977) kamen zu der gleichen Ansicht, wohingegen Olsman und KROL (1972) hinter der allgemeineren Hypothese - Bindung nur eines NOMoleküls - stehen.

Das Verbleichen der Farbe ist eine Folge der Luft- (Sauerstoff) und Lichteinwirkung. Bei im Kaltverfahren hergestellten Fleischwaren bewirkt schon Sauerstoff allein einen Zerfall der Farbverbindung; bei erhitzter Ware ist hierzu ein Zusammenwirken von Sauerstoff und Licht erforderlich (RHODES 1976, mündliche Information). TARLAGDIS (1962) vertritt denn auch die Auffassung, dass bei nicht wärmebehandeltem Fleisch der Farbstoff je nach pH nur teilweise $\mathrm{zu}$ Chromogen denaturiert worden ist.

Ein Teil des Fleischpigments kann ausserdem u.U. in Form von Stickoxidmetmyoglobin oder Metmyoglobin erhalten bleiben, das durch Denaturierung in graues Metmyochromogen umgewandelt wird. WALSH und Rose (1956) haben festgestellt, dass es im Dunkeln zu einer langsamen Oxydation von Stickoxidmyoglobin gemäss folgender Gleichung (4) kommt:

$$
\mathrm{NOMb}+1 / 2 \quad 0_{2} \underset{\mathrm{v}_{2}}{\stackrel{\mathrm{v}_{1}}{\rightleftarrows}} \mathrm{MMb}+\mathrm{NO}_{2}
$$

Die Reaktionsgeschwindigkeit $\mathrm{v}_{\mathbf{1}}$ der Gleichung (4) ist unabhängig vom $\mathrm{pH}$, nimmt aber mit steigender Temperatur zu. Durch Lichteinwirkung wird diese Umsetzung stark beschleunigt. Setzt man jedoch dem Reaktionsgemisch Nitrit zu, so verläuft die Oxydation im Dunkeln bis herunter zu pH 6,3 langsam, wird aber dann bei noch weiterem $\mathrm{pH}$-Abfall stark beschleunigt. Unter Lichteinwirkung verläuft diese Reaktion auch bei hohen $\mathrm{pH}$-Werten (Bild 2) sehr rasant. Der Einfluss des Nitritgehaltes ist aus Bild 3 ersichtlich (WaLsh und Rose 1956). Allerdings ist zu berücksichtigen, dass in der Rohwurst infolge des niedrigen $\mathrm{pH}$-Werts kein oder nur noch wenig freies Nitrit vorhanden 
ist (MIRna 1971) und der Grossteil des Farbstoffs in Chromogenform vorliegt (NinnivaARA 1955). Aus dem Vorangehenden kann gefolgert werden, dass Rohwurst mit hohem $\mathrm{pH}$ wegen der geringeren Chromogenbildung eine schlechtere Farbbeständigkeit als solche mit niedrigem $\mathrm{pH}$ aufweist (Bild 2 und 3).

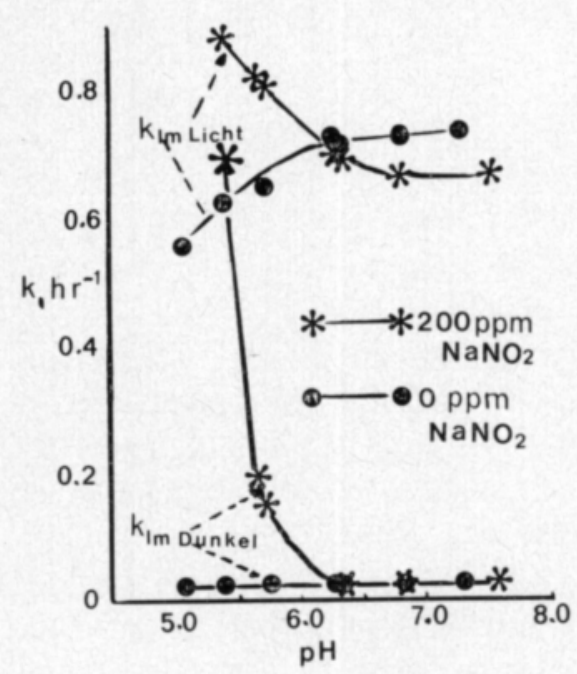

Bild 2. Einfluss des pH-Werts auf die Stickoxidmyoglobin-Oxydationsgeschwindigkeit bei und ohne Anwesenheit von Nitrit (WALsH und Rose 1956).

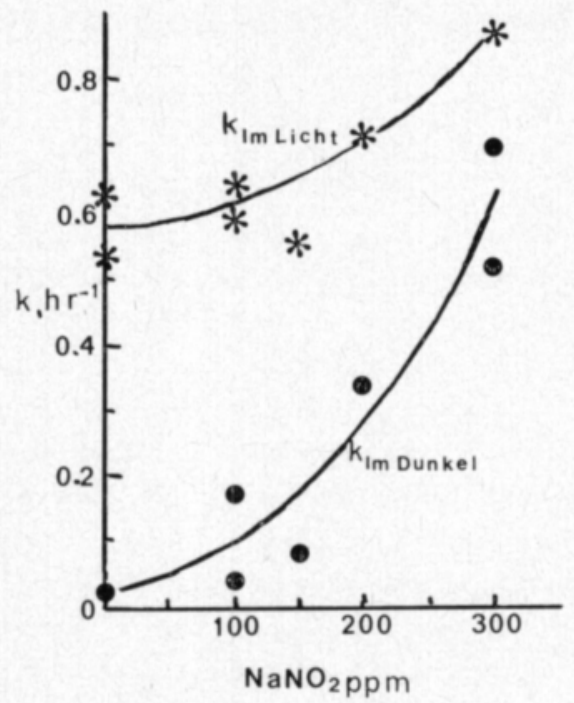

Bild 3. Einfluss der Nitritkonzentration auf die Stickoxidmyoglobin-Oxydationsgeschwindigkeit bei pH 5,6 (WaLsh und Rose 1956).

Die Stabilität des bereits gebildeten Stickoxidmyochromogens nimmt hingegen mit steigendem $\mathrm{pH}$ zu. Die Farbverblassung geschieht in zwei Phasen: Zunächst wird durch Lichteinwirkung das Stickoxid vom Häm getrennt, danach wird es vom Nitrit oder Sauerstoff oxydiert. In sauerstofffreiem Milieu tritt keine Farbverblassung ein. Auch bei Einwirkung von Sauerstoff kann es, vorausgesetzt es sind Reduktionsmittel vorhanden, zu einer langsamen Farbneubildung kommen. Ist das Nitrit bzw. irgendein anderes Oxydationsmittel (z.B. Peroxid) in reichlicher Menge vorhanden, so wird das Pigment zu einem grünen Porphyrin-Ring oxydiert (TARLAgdis 1962, Fox und Thomson 1964, Review: Fox 1966).

Die Geschwindigkeit des Verbleichens steigt mit sinkendem $\mathrm{pH}$ und zunehmendem Nitritgehalt, und zum Schluss kommt es u.U. zur Bildung farbloser oder gelblicher Verbindungen. Die grüne Verbindung lässt sich nicht wieder in rote zurückführen; bei Einwirkung von Reduktionsmitteln erfolgt ein Umschlag in kräftigeres Grün (Review: Fox 1966). Mit Hämochromen liessen sich keine entsprechenden Versuche durchführen, jedoch kann vorausgesetzt werden, dass die Umsetzungen in etwa gleicher Weise erfolgen (Review: LAWRIE 1974).

Die Farbverblassung kann durch Reduktionsmittel und kleinen Nitritüberschuss verzögert werden (TARLAGDIS 1962, REITH und SZAKÁLy 1967 b). Das Nitrit liegt hierbei offensichtlich als Stickoxid, gebunden oder als freie Nitrit-Ionen vor. Salpetrige Säure würde den Farbstoff oxydieren. 
Das in Fleischwaren enthaltene Nitrat muss, bevor es die gewünschten Wirkungen ergibt, zu Nitrit reduziert werden (NIInIvaARA 1955). Da Fleisch keine nitratreduzierenden Enzyme enthält (MöHLER 1974), muss die Reduktion auf bakterieller Basis erfolgen. Zahlreiche Vertreter z. B. der Gattungen Micrococcus und Bacillus sowie viele gramnegative Bakterien reduzieren Nitrat zu Nitrit, jedoch lässt sich bei den einzelnen Gattungen in dieser Hinsicht keine deutliche Regelgebundenheit feststellen (BERgey's Mandal 1974). Wichtig für die bei der Rohwurstherstellung verwendeten Starterkulturen ist ihr Nitratreduktionsvermögen (РонJA 1960), das sich auch beim Arbeiten mit Nitrit vorteilhaft auswirkt, weil ein Teil des Nitrits zu Nitrat oxydiert wird (MöHLER 1970). Besonders wichtig ist das Nitratreduktionsvermögen beim Arbeiten mit verringerten Nitritzusätzen, da dann der relative Anteil an Nitrat so anwachsen kann, dass, wird das entstehende Nitrat im Laufe des Herstellungsprozesses nicht zu Nitrit zurückreduziert, nicht mehr genügend Nitrit für die Umrötung vorhanden ist. Ein Einsatz reiner Nitratreduktase oder auch nur roher Extrakte als Ersatz für lebende Organismen ist bei Rohwurstbrät ohne Erfolg geblieben (PFEIL und LIEPE $1973 \mathrm{a}$ ).

Der im Hinblick auf das Nitratreduktionsvermögen der Bakterien optimale $\mathrm{pH}$ beträgt nach Schormüller und Schilling $\left.\left(1961^{1}\right), 1963^{2}\right)$ ) 7,0, nach Pfeil und Liepe $\left(1973 \mathrm{~b}^{3}\right)$ ) 7,5, und die diesbezügliche Aktivität nimmt diesen Autoren zufolge mit sinkendem pH-Wert rasch ab. Puolanne u.a. $\left(1977^{4}\right)$ ) ermittelten im Zusammenhang mit Lösungsversuchen den optimalen $\mathrm{pH}$ zu 6,0 und stellten auch unterhalb von $\mathrm{pH} 5,0$ noch eine hohe Aktivität fest. Nach NinnivaARa (1955) findet unterhalb von $\mathrm{pH} 5,4$ weder in Bouillon noch in Wurst Nitratreduktion mehr statt. Im Lichte dieser Ergebnisse betrachtet ist offensichtlich, dass bei Rohwurst durch $\mathrm{pH}$-Abfall andere das Reduktionsvermögen mitbestimmende Faktoren stärker beeinflusst werden als die Nitratreduktase selbst (Pfeil und Liepe 1973 b, Puolanne u.a. 1977).

Nach Puolanne u.a. (1977) ist das Nitratreduktionsvermögen von Micrococcus sp. unterhalb $10^{\circ} \mathrm{C}$ schwach und steigt dann bis zu einer Temperatur von $44^{\circ} \mathrm{C}$ gleichmässig an; der Salzgehalt hat hingegen keinen entscheidenden Einfluss auf das Nitratreduktionsvermögen der Mikrokokken. Auch fanden sie, dass die $\mathrm{K}_{\mathrm{m}}$-Werte der Nitratreduktase im Optimalbereich der einzelnen Faktoren (Temperatur, $\mathrm{pH}$, Salzgehalt) zwischen 8,7 und 14,1 ppm $\mathrm{NaNO}_{3}$ variierten. Die nahezu maximale Reduktionsgeschwindigkeit wird demzufolge schon bei sehr niedriger Nitratkonzentration erreicht. Bei einer Nitratkonzentration von $100 \mathrm{ppm}$ (entspricht etwa $50 \mathrm{ppm}$ bei Wurst mit $\mathbf{5 0} \%$ Wassergehalt) beträgt die Nitratreduktionsgeschwindigkeit $87 \%$ der Geschwindigkeit bei 600 ppm.

Daraus lässt sich schliessen, dass bei Rohwurst die Nitratkonzentration anfangs keinen wesentlichen Einfluss auf die Nitratreduktionsgeschwindigkeit hat, so dass zumindest in dieser Hinsicht einer Senkung der Nitratkonzentration nichts im Wege steht.

1) Micrococcus caseolyticus $I I I / 2$.

2) Micrococcus epidermidis var. albus.

3) Micrococcus sp. (Baktoferment 61).

4) Micrococcus $s p$. 
Bakterien vermögen auch Nitrit zu reduzieren (BERGEY's MANUAL 1974). AкAshi (1974 a und b) stellte fest, dass die Stämme, die weniger $\mathrm{NH}_{3}$ und mehr NO als die übrigen Stämme produzierte im Zusammenhang mit dem Pökeln von Fleischwaren eine günstige Wirkung ausübten. Auch nach PFEIL und LIEPE (1974) wird Nitrit durch Starterkulturen reduziert.

\section{2 Technologie des Nitrit- und Nitrateinsatzes bei Rohwurst}

Nitrat gelangte ursprünglich als Verunreinigung zusammen init Kochsalz in die Fleischerzeugnisse (LAWRIE 1974). Später ging man dann dazu über, es mit Absicht und gleich in grossen Mengen - bis zu $1 \%-$ zuzusetzen. Im allgemeinen lagen die zugesetzten Mengen zwischen 500 und $3000 \mathrm{ppm}$ (BRAATHEN 1963). Farbfehler führte man in der Regel auf ungleichmässige Salzverteilung und unzureichende Nitratkonzentration zurück und versuchte entsprechend, mangelhafte Umrötung durch erhöhte Nitratzugabe auszugleichen. Da man inzwischen die aktive Rolle des Nitrits (Stickoxid) bei der Umrötung und die bakterielle Nitratreduktion zu Nitrit erkannt hatte, begann man den Fleischerzeugnissen auch Nitrit zuzusetzen (BROOKS 1937, LAwriE 1974, Review: Binkert und Kolari 1975).

Beim Übergang auf nitratreduzierende Starterkulturen bzw. direkten Nitritzusatz arbeitete man zunächst weiterhin mit grossen Zusatzstoffmengen und oft mit erhöhten Reifetemperaturen. Die Folge waren häufig auf zu hohe Nitritkonzentration und zu niedrigen $\mathrm{pH}$ zurückzuführender Nitritbrand ("nitrite burn") (Fox und TномsоN 1964) oder grüne Farbfehler infolge zu hoher Anzahl Laktobazillen bzw. anderer peroxidbildender und katalasenegativer Bakterien (Nurmi 1966 a). Nachdem dann die Umrötungstheorie allgemeinen Eingang gefunden hatte, begann man die Nitrit- und Nitratzusätze zu verringern; technologische Nachteile ergaben sich daraus keine, im Gegenteil, die Nitritbrand-Gefahr schien eher abzunehmen. Gleichzeitig hatte man dabei auch die durch Nitrit und Nitrat gegebene akute Toxizitätsgefahr im Auge.

Aus dieser Periode liegen nur ganz wenig Untersuchungsergebnisse über den Einfluss der zugesetzten Nitrit- und/oder Nitratmengen auf die Umrötung, dafür aber zahlreiche Arbeiten über die Wirkung dieser Stoffe auf das Wachstum pathogener Bakterien vor (INGRAM 1974).

In vielen Ländern ist der gleichzeitige Zusatz von Nitrit und Nitrat verboten. Nitrit darf ausschliesslich als 0,6prozentige $\mathrm{NaCl}$-Mischung zugesetzt werden. Diese Einschränkung führte allgemein zu einenı Nitratzusatz von $500 \mathrm{ppm}$ bzw. zu einer Nitritmenge von $150-180 \mathrm{ppm}$ (Proc. ISNMP 1. 1974). Nach Bekanntwerden des Nitrosamin-Problems begann man in allerletzter Zeit erneut Untersuchungen über die Mindestkonzentrationen anzustellen, mit denen sich noch einwandfreie Ware gewohnter Qualität herstellen lässt. Inzwischen war allerdings auch dank dem Einsatz von Starterkulturen, GdL und Askorbinverbindungen sowie verbesserter Hygiene und Reifungstechnologie der Reifeprozess, namentlich was die Umrötung betrifft, leichter unter Kontrolle zu halten. Es zeigte sich nun, dass der begrenzende Faktor bei der Verringerung der Nitrit- bzw. Nitratzugabe nicht, wie früher angenommen, 
die Umrötung, sondern vielmehr die Zunahme des mikrobiologischen Risikos ist (Leistner u.a. 1973 a und b, Wirth 1973).

Bei der Diskussion von Farbfehlern ging man allgemein von der Zusammensetzung der Mikrobenflora aus - früher die einzige Möglichkeit, an dieses Problem heranzugehen, da die verwendeten Nitrit- oder Nitratmengen auf jeden Fall für die Umrötung ausreichten. Nach NirnivaAra (1955) ist bei ungenügender oder fehlender bakterieller Nitratreduktion eine unvollständige Umrötung die Folge. Eine zu heftige Reduktion wiederum führt zu NitritbrandErscheinungen (BAcus und Derbel 1972). Gewisse Milchsäurebakterien erzeugen Wasserstoffperoxid, welches Myoglobin zu grünen Porphyrinverbindungen oxydiert (Grever und Schuddenboom 1958). Das in der Rohwurst enthaltene Nitrit hemmt die Katalasen in ihrer Aktivität, und mit sinkendem $\mathrm{pH}$-Wert nimmt diese Hemmwirkung zu, wie im allgemeinen im Zusammenhang mit Hämverbindungen (WALTERS u.a. 1974). Weiter ist auch bei Rückgang des $\mathrm{pH}$-Wertes (Optimum $\mathrm{pH} \mathbf{8 , 0}$ ) und bei Zunahme des Salzgehaltes eine Abnahme der Katalasenaktivität des Fleisches zu beobachten. Der optimale pH-Wert für Mikrobenkatalasen beträgt 5,0, wodurch der Abfall der FleischKatalasenaktivität teilweise kompensiert wird (RozIER 1971). Farbfehler werden u.a. von Lactobacillus viridescens, Lactobacillus plantarum sowie von zahlreichen anderen Lactobacillus- und Streptococcus-Arten verursacht (Review: Nurmi 1966 a). Nurmi (1966 a) stellte fest, dass beim Arbeiten mit Nitrat (468 ppm) Laktobazillen ${ }^{1}$ ) allein Farb- (grün) und Geschmacksfehler bewirkten, die mit wachsender Laktobazillenzahl immer ausgeprägter wurden. Bei ausschliesslichem Zusatz von Mikrokokken²) war die Folge eine bräunliche Färbung, wohingegen mit einem Laktobazillen-Mikrokokken-Gemisch (1:1, ca. $10^{7} \mathrm{Keime} / \mathrm{g}$ ) eine gute, beständige Farbe erzielt wurde und die Würste innerhalb kurzer Zeit Konsistenz und Aroma reifer Ware erreichten. Beim Arbeiten mit Nitrit $(200 \mathrm{ppm})$ traten auch bei der Laktobazillen-Wurst keine Farbfehler auf, wohl aber Geschmacksfehler. Bei Wurst vom amerikanischen Typ wird meist Nitrit in Verbindung mit Laktobazillen-Starterkulturen eingesetzt, seltener hingegen Nitrat (DEIBEL 1974).

Von Reuter (1972) liegt eine umfangreiche Studie über den Einsatz von Bakterienreinkulturen - in erster Linie von Laktobazillenstämmen - sowie von GdL zusammen mit Nitrit und Nitrat bei der Wurstherstellung vor. Die Mikrokokken ${ }^{2}$ ) verhinderten beim Arbeiten mit Nitrat und GdL die Bildung von Farbfehlern. Reuter konstatierte allerdings, dass, gehören die in der Wurst enthaltenen Laktobazillen nicht zu den farbfehlerverursachenden Arten, die Mikrokokken keine deutliche Farbverbesserung bewirken. Die mit Mikrokokken-Starterkulturen hergestellten Würste wiesen im allgemeinen ein dunkleres Rot als die Vergleichswürste auf. GdL eignete sich am besten im Zusammenhang mit Nitrit, und durch Zusatz von Mikrokokken-Starterkulturen liessen sich die mangelhafte Abrundung und Stabilität der GdL-Würste zum Teil verbessern. Weiter stellte Reuter fest, dass sich durch Wahl eines für Rohwurst geeigneten Laktobazillen-Stammes Geschmacks- und Farbfehler

1) Lactobacillus plantarum.

2) Micrococcus sp. 
vermeiden lassen, denn seinen Darlegungen zufolge scheiden nur gewisse heterofermentative Stämme Peroxid ab. Er kommt somit im Gegensatz zu Nurmi (1966 a) zu dem Schluss, dass die Peroxidbildung hier von untergeordneter Bedeutung ist.

LAwrowa u.a. (1967) haben Untersuchungen über die zur Erzielung einer beständigen Farbe bei Rohwurst erforderlichen Mindestkonzentrationen angestellt. Ihren Ausführungen zufolge genügte für Wurst vom SummerSausage-Typ ein Nitritzusatz von 75 ppm; der Restnitritgehalt betrug dann $30 \mathrm{ppm}$, d.h. 1/5-1/10 der damals in der Sowjetunion üblichen Menge.

TJABERG u.a. (1969) setzten der Rohwurst verschiedenartige Farbstoffe zu; trotzdem verursachten die Laktobazillen Farbfehler. Bei gleichzeitigem Einsatz von Laktobazillen ${ }^{1}$ ), Mikrokokken²), Nitrit und Nitrat sowie Farbstoff traten keine Farbfehler auf.

Mirna (1973) untersuchte die Eigenschaften von Rohwurst mit üblichem (126 ppm $\mathrm{NaNO}_{2}$ ) und mit verringertem (31,5 ppm $\mathrm{NaNO}_{2}$ ) NitritpökelsalzZusatz. Beim Arbeiten mit der üblichen Menge betrug der Gehalt an freiem Restnitrit nur 3 ppm und die Gesamtnitritmenge (gebundenes Nitrit + Nitrat) 24 ppm; vom Myoglobin entfielen $73 \%$ auf Stickoxidmyoglobin. Bei der Wurst mit dem verringerten Nitritzusatz lauteten die entsprechenden Werte: $2 \mathrm{ppm}, 10 \mathrm{ppm}$ und $64 \%$. Bei Zugabe von Askorbat (500 ppm) oder GdL $(5000 \mathrm{ppm})$ ging bei der letzteren Wurst die Restnitritmenge kaum noch zurück, dafür kam es zu einem kräftigen Anstieg der Nitratmenge. Der relative Stickoxidmyoglobin-Anteil betrug $78 \%$ (Askorbat-Wurst) bzw. $81 \%$ (GdL-Wurst). Daraus ergab sich die Feststellung, dass sich bei Einsatz von Askorbat oder GdL auch mit geringem Nitritzusatz eine ausgezeichnete Umrötung erzielen lässt. Nach MöHLER (1959) liegt bei Rohwurst, bedingt durch den niedrigeren $\mathrm{pH}$, der Stickoxidmyoglobin-Anteil höher als bei Brühwurst. In seinen Versuchen betrug der Stickoxidmyoglobin-Anteil je nach Wursttyp zwischen 74 und $93 \%$. Durch Einsatz von Askorbinsäure wurde ein geringfügig besseres Ergebnis erzielt.

Pfeil und Liepe (1973 b) fanden, dass sowohl bei Nitrit- als auch bei NitratEinsatz durch Zusatz von Starterkultur ${ }^{2}$ ) eine Verringerung des Restnitritbzw. Restnitratgehalts erzielt wurde. Verringerte man den Nitratzusatz von $500 \mathrm{ppm}$ auf $200 \mathrm{ppm}$, so sank der Restnitritgehalt auf unter die Hälfte. Bei Einsatz von $200 \mathrm{ppm}$ Nitrat und Starterkultur lag der Nitritgehalt während der gesamten Versuchsdauer niedriger als bei den mit 500 ppm Nitrat oder 180 ppm Nitrit hergestellten Würsten.

Gerhard und Haller (1973) untersuchten den Einfluss von GdL auf die Restnitrit- und -nitratmengen und auf den Stickstoffoxidmyoglobin-Anteil bei verschieden grossen Nitrit- und Nitratzusätzen; sie verwendeten dabei keine Starterkulturen. Wie sie feststellten, blieb der Stickoxidmyoglobin-Anteil bei Einsatz von GdL und Zusatz von $10-28 \mathrm{~g} / \mathrm{kg}$ Nitritpökelsalz (theoretischer Wert 55-154 ppm $\mathrm{NaNO}_{2}$ ) praktisch konstant. Durch Zusatz von Askorbin-

1) Lactobacillus plantarum.

2) Micrococcus sp. (Baktoferment 61). 
säure wurde keine weitere Farbbildung erzielt. Bei den GdL-freien NitratWürsten (500 ppm $\mathrm{KNO}_{3}$ ) und Nitrit-Würsten (154 ppm $\mathrm{NaNO}_{2}$ ) lag der Stickoxidmyoglobin-Anteil um 10-15 Prozentpunkte niedriger als bei den GdL-Würsten. Letztere Würste wiesen trotz verschieden grossen Nitritzusätzen bezüglich der Restnitrit- und -nitratmenge keine erheblichen Unterschiede auf, enthielten aber durchweg weniger Restnitrit und -nitrat als die ohne GdLZusatz hergestellten Würste.

Detmers u.a. (1975) setzten Thüringer-Wurst zusammen mit Starterkulturen $\left.{ }^{1}\right) 0-125 \mathrm{ppm} \mathrm{NaNO}_{2}$ und $0-1500$ ppm $\mathrm{KNO}_{3}$ zu und stellten fest, dass der Nitrit- und Nitratgehalt während der Lagerung zurückging. Die Nitratabnahme war bei hoher Temperatur $\left(+27^{\circ} \mathrm{C}\right)$ ausgeprägter als bei niedriger $\left(+7,5^{\circ} \mathrm{C}\right)$; auf den Nitritschwund hatte die Temperatur keinen Einfluss.

Nach SkJelkvåle u.a. (1974) wiesen Würste mit 0 ppm und 164 ppm Nitrit keine signifikanten geschmacklichen Unterschiede auf sofern die Herstellung unter Einsatz von Starterkulturen ${ }^{2}$ ) oder GdL erfolgte. Beim Arbeiten ohne Starterkulturen und GdL wurde zwischen den Würsten mit $164 \mathrm{ppm}$ und 0 ppm $\mathrm{NaNO}_{2}$ ein signifikanter Unterschied festgestellt, nicht mehr aber zwischen den Würsten mit $82 \mathrm{ppm}$ und $0 \mathrm{ppm} \mathrm{NaNO}_{2}$. Detmers u.a. (1975) hingegen konstatierten, dass ohne Nitrit- und Nitratzusatz hergestellte Würste deutlich einen fehlerhaften, ranzigen Geschmack aufwiesen, dass aber schon durch Zugabe von $50 \mathrm{ppm} \mathrm{NaNO}_{2}$ oder $500 \mathrm{ppm} \mathrm{KNO}_{3}$ diese Fehler vermieden wurden. Die gleichen Zusatzstoffmengen bewirkten ausserdem eine signifikante Verbesserung des Geschmacks und Aussehens der Ware. Durch Erhöhung des Nitritzusatzes auf 100 oder $150 \mathrm{ppm}$ wurden - organoleptisch beurteilt - noch geringfügig bessere Eigenschaften erzielt; zusätzliche Nitratzugabe führte hingegen zu keinem günstigeren Resultat.

Nestorov u.a. (1975) stellten bei Zusatz von 1000 ppm $\mathrm{KNO}_{3}, 250 \mathrm{ppm}$ $\mathrm{KNO}_{3}$ oder $80 \mathrm{ppm} \mathrm{NaNO}_{2}$ zusammen mit Starterkulturen ${ }^{3}$ ) bezüglich der Stickoxidmyoglobin-Menge keine wesentlichen Unterschiede fest. Die grösste Stickoxidmyoglobin-Menge wurde bei gleichzeitigem Einsatz von $80 \mathrm{ppm}$ $\mathrm{NaNO}_{2}$ und 500 ppm Askorbat erreicht. Die Nitrat-Würste hatten keinen Askorbatzusatz erhalten.

PetÄJÄ (1977) hat eine umfangreiche Arbeit über die Verwendung von gramnegativen Bakterien als Starterkulturen in der Rohwurst durchgeführt. Er hat insgesamt 138 Versuchswurstposten mit einem Zusatz von nur 200 ppm $\mathrm{KNO}_{3}$ hergestellt, und in keinem Falle wurden durch diesen niedrigen Nitratzusatz Farbfehler verursacht. Der Nitritgehalt der Würste lag gewöhnlich unter $10 \mathrm{ppm}$; in drei Tage alten Würsten variierte er jedoch je nach Starterkulturen zwischen 9 und $57 \mathrm{ppm}$. Der niedrigste Wert wurde durch Verwendung von Aeromonas $s p$. und Lactobacillus plantarum ${ }^{4}$ ) als Starterkulturen, der höchste Wert mit einem anderen Aeromonas-Stamm erreicht.

\footnotetext{
1) Lactobacillus plantarum und Pediococcus cerevisiae (Lactacel MC).

2) Lactobacillus plantarum und Micrococcus sp. (Duploferment 66)

3) Micrococcus P4.

4) Aus Duploferment 66
} 
Nach Meinung des Verfassers beruht dies darauf, dass der erstgenannte Aeromonas-Stamm ein schwacher Nitratreduzierer ist, und ausserdem durch Einsatz von Laktobazillen das Nitratgehalts-Maximum gesenkt wurde. Nach 21 Tagen waren die Unterschiede zwischen den Würsten unbedeutend.

Wirth $(1973,1974)$ stellt zusammenfassend über seine Untersuchungen fest, dass eine Rohwurstherstellung ohne Nitrit oder Nitrat nicht möglich ist, und dass eine gute, beständige Farbe durch Zusatz von $60-80 \mathrm{ppm} \mathrm{NaNO}$ oder $200 \mathrm{ppm} \mathrm{KNO}_{3}$ erreicht wird. Als Mindestmengen gibt er $40 \mathrm{ppm} \mathrm{NaNO}_{2}$ und $150 \mathrm{ppm} \mathrm{KNO}_{3}$ an, wobei es nach seiner Auffassung in der industriellen Produktion bereits zu vereinzelten Farbfehler-Fällen kommen kann. Beim Arbeiten mit Nitrit sichern $\mathbf{3 0 0}-\mathbf{5 0 0}$ ppm Askorbat, beim Arbeiten mit Nitrat 100-200 ppm Askorbat die Umrötung. In geschmacklicher Hinsicht gestaltet sich hingegen ein niedriger Nitritzusatz günstiger, und sogar $30 \mathrm{ppm}$ ergeben den für Rohwurst typischen Geschmack. Gewöhnlich ergaben jedoch $30 \mathrm{ppm}$ einen sauren Geschmack. Die Restnitrit- und -nitratgehalte lagen in seinen Untersuchungen sehr niedrig. Die Geschmacksverbesserung führt man auf die höhere Aktivität der aromabildenden Bakterien bei niedrigen Nitritkonzentrationen zurück.

Mirna und Coretti (1974) konnten mit 156 und 15,6 ppm schwarzem Roussin'schem Salz (sRS) eine braunrötliche Farbe auf Rohwurst erreichen. In Kulturversuchen zeigte sRS starke bakteriostatische Wirkung gegen Mikrokokken und Enterobacter-Keime, aber der Hemmeffekt wurde in Anwesenheit von Fleischproteinen teilweise wieder aufgehoben.

\section{3 Pathogene Bakterien in der Rohwurst}

RöDEL u.a. (1975) haben ein Verfahren zur Einteilung von Fleischwaren in Haltbarkeitsgruppen auf Grund ihres $\mathrm{a}_{w^{-}}$und pH-Wertes dargelegt, demzufolge Fleischwaren mit einem $\mathrm{a}_{\mathrm{w}}$-Wert $\leq 0,95$ und einem $\mathrm{pH}$-Wert $\leq 5,2$ oder einem $\mathrm{a}_{\mathrm{w}}$-Wert $<0,91$ oder einem $\mathrm{pH}$-Wert $<\mathbf{5 , 0}$ auch ohne Kaltlagerung haltbar sind. Die Haltbarkeit von Fleischerzeugnissen wird von zahlreichen Faktoren mitbestimmt, die bei der Festlegung der oben angeführten Grenzwerte zum Grossteil indirekt Berücksichtigung fanden. Allerdings gelten diese Grenzwerte nur für den bakteriellen, nicht aber für den chemischen sowie den durch Schimmelpilze und Hefen bedingten Verderb, der auf andere Weise unter Kontrolle gehalten werden muss.

Rohwurst erfüllt im allgemeinen die o.g. Bedingungen, wobei freilich im Hinblick auf das Pathogenitätsrisiko zwei Einschränkungen zu machen sind. In der Arbeit von RöDEL u.a. (1975) ging es ausschliesslich um den Verderb der Ware, und abnormal starke Kontaminationen durch pathogene Bakterien wurden in den Versuchen offensichtlich nicht festgestellt. Nahrungsmittelvergiftung ist stets durch Abweichung vom Normalen bedingt; bei Rohwurst kommt als solche in erster Linie eine starke Anfangskontamination in Frage, denn die übrigen Einflussfaktoren können als konstant oder nur beschränkt variabel betrachtet werden. Nun herrschen bei der Rohwurstherstellung je nach Wursttyp und Technologie mehrere Tage lang Verhältnisse, unter denen sich pathogene Bakterien vermehren und auch Toxine bilden können, und in 
dieser Periode müssen auch verschiedene andere Einflussfaktoren als Variable berücksichtigt werden, darunter der Nitritgehalt. Entscheidend in dieser dynamischen Phase der Produktion ist die gemeinsame Wirkung aller eine bestimmte Eigenschaft beeinflussenden Faktoren, so dass also, wenn einer dieser Faktoren in seiner Wirkung nachlässt (beispielsweise bei Verringerung des Nitritzusatzes), der Anteil der übrigen Begrenzungsfaktoren entsprechend erhöht werden muss (Hürdeneffekt, RöDEL u.a. 1975). Was die pathogenen Bakterien in der Rohwurst betrifft, so handelt es sich um eine Risikoeinschätzung, bei der die wahrscheinliche Anfangskontamination und die Variationsmöglichkeiten der Verfahrenstechnik zu berücksichtigen sind. Gegenwärtig sind allgemeine Bestrebungen im Gange, den Nitritzusatz zu senken und den Anteil der übrigen Begrenzungsfaktoren zu erhöhen ohne jedoch das Pathogenitätsrisiko zu stark anwachsen zu lassen und die Qualität des Endprodukts zu beeinträchtigen.

\section{Salmonellen}

Salmonellen werden hin und wieder auch in Rohwurst festgestellt (MARAzza und Crespi 1963, Ortmann 1967, Takács und Simonffy 1970, Tarnay 1975); der wichtigste Faktor bei der Begrenzung ihres Wachstums ist der $\mathrm{pH}$. Nach einer Studie von Goepfert und Chung (1970) beeinflussten sowohl der pH als auch der Salzgehalt das Wachstum von Salmonella typhimurium; Nitrit zeigte weder allein noch zusammen mit Salz bakterizide Wirkung. Eine pHSenkung auf 4,3 hatte hingegen ein schnelles (24 Stdn.) Absterben der Salmonellen zur Folge. Zahlreiche Forscher haben die Vernichtung der Salmonellen als Folge des durch Starterkulturen bedingten $\mathrm{pH}$-Abfalls konstatiert (CoEPFERT und Chung 1970¹), Gilliland und SPECK 1972²), Leistner u.a. 1973 b $^{3}$ ), Sмıтн u.a. $1975 \mathrm{a}^{4}$ ), Sirviö u.a. $\left.1977^{5}\right)$ ). Auch die Säure, die den pH-Rückgang bewirkt, ist bei der Hemmung des Salmonellenwachstums von Bedeutung (Chung und Goepfert 1970).

Lösungsversuche ergaben beim Arbeiten mit Essigsäure für das Wachstum von Salmonellen einen pH-Mindestwert von 5,40; für Milch- und Salzsäure betrugen die entsprechenden Werte 4,40 bzw. 4,05. Nach Leistner u.a. (1973 b) hemmte auch GdL das Wachstum von Salmonellen und bewirkte zusammen mit Nitrit deren Absterben, was auf die vom GdL verursachte $\mathrm{pH}$ Abnahme zurückzuführen war. Durch Räuchern und Trocknen der Wurstoberfläche werden die auf letzterer befindlichen Salmonellen abgetötet (SснEIBNER 1969).

Nach TAKÁcs und Simonffy (1970) werden die Salmonellen in der ungarischen Salami durch den hohen Salzgehalt nicht vollständig abgetötet, sondern es ist wegen des hohen pH-Wertes (5,8-6,0 am Ende des Reifeprozesses) stets eine gewisse Gefahr latenter Salmonelleninfektion vorhanden.

\footnotetext{
1) Lactobacillus sp. und Pediococcus sp.

${ }^{2}$ ) Streptococcus $V T_{3}$.

3) Lactobacillus $s p$.

4) Lactobacillus plantarum und Pediococcus cerevisiae (Lactacel MC).

$\left.{ }^{5}\right)$ Lactobacillus plantarum und Micrococcus sp. (Duploferment 66 und Baktoferment 61).
} 
Leistner u.a. (1973 b) haben das Verhalten der Salmonellen in Rohwurst hauptsächlich in Abhängigkeit vom Nitrit eingehend untersucht. Dabei arbeiteten sie mit einer Mischung aus zehn verschiedenen Salmonella-Stämmen. Als Variable fungierten Nitritkonzentration, GdL, Starterkulturen ${ }^{\mathbf{1}}$ ) und Umfang des Salmonellenzusatzes.

Im Gegensatz zu Goepfert und Chung (1970) stellten Leistner u.a. (1973 b) fest, dass Nitrit eine wachstumshemmende Wirkung auf Salmonellen ausübt: Ein Nitritzusatz von 135 bzw. 101 ppm hatte bei hohem SalmonellenZusatz $\left(10^{6} \mathrm{Keime} / \mathrm{g}\right)$ eine Hemmung deren Wachstums, bei niedrigem Salmonellen-Zusatz ( $10^{2}$ Keime/g) deren Absterben zur Folge. Auch Sirviö u.a. (1977) konstatierten eine wachstumshemmende Wirkung des Nitrits auf Salmonellen. Bei Nitrat war ein solcher Effekt nur im Zusammenhang mit Starterkulturen zu beobachten.

Falls bei der Rohwurstherstellung nicht mit Nitrit oder Nitrat gearbeitet und gleichzeitig für pH-Senkung, hohen Salzgehalt und Räuchern gesorgt wird, ist die Möglichkeit einer raschen Vermehrung der Salmonellen durchaus gegeben (Leistner u.a. 1973 b, Sirviö u.a. 1977). Bei der Einschätzung des durch Salmonellen bedingten Risikos auf der Basis der verfügbaren Untersuchungsergebnisse sind eine ganze Anzahl der oben aufgezählten Faktoren (Salmonella-Stamm, Ausmass der Kontamination, Nitrit- bzw. Nitratkonzentration, Salzgehalt, Zuckergehalt, Reifetemperatur, Einsatz von LaktobazillenStarterkultur und GdL sowie anderen Zusatzstoffen) zu berücksichtigen. Zusammenfassend kann hierzu festgestellt werden, dass bei Rohwurst ein Zusatz von wenigstens $100 \mathrm{ppm}$ Nitrit und eine Senkung des $\mathrm{pH}$-Wertes durch Starterkulturen oder GdL auf 5,2 oder darunter erforderlich sind (LEIsTNER u.a. 1973 b).

\section{Staphylococcus aureus}

Staphylococcus aureus ist zwar in Rohwurst häufig anzutreffen (BARBER und Deibel 1972), aber in den allermeisten Fällen in so geringen Mengen, dass keine Gefahr einer Lebensmittelvergiftung besteht (RIEMANN u.a. 1972, NisKANEN und Nurmi 1976). Trotzdem sind Fälle von Staphylokokken-Vergiftung durch Rohwurstverzehr bekannt (NCDC 1971 a und b). Bei Rohwurst reicht selten ein Einzelfaktor aus, um Wachstum oder Toxinbildung von S. aureus zu verhindern, so dass durch Versuche, die nur einen Teil der Einflussfaktoren berücksichtigen (Review: Riemann u.a. 1972, Buchanan und Sohlberg 1972, Genigeorgis u.a. 1971, Troller und Stinson 1975), lediglich diejenigen Umstände klargestellt werden, unter denen Wachstum bzw. Toxinbildung in der Rohwurst als gehemmt vorausgesetzt werden kann.

Das Wachstum bzw. Absterben und die Toxinbildung von S. aureus werden gleichzeitig von wenigstens folgenden Faktoren beeinflusst, die während des Rohwurst-Herstellungsprozesses ausserdem ständigen Änderungen unterworfen sind:

\footnotetext{
1) Lactobacillus sp.
} 
- Staphylococcus-Stamm

- Toxin (A, B oder C)

- pH-Wert

- Temperatur

- Redox-Potential

- Ausmass der Kontamination

- Salzgehalt

- Nitrit- und Nitratgehalt

- Räuchern

- sonstige Zusatzstoffe (vor allem GdL)

- Starterkulturen und endogene Flora

Im neutralen $\mathrm{pH}$-Bereich bewirkt eine Abnahme des $\mathrm{a}_{\mathrm{w}}$-Wertes bis herunter zu $\mathrm{a}_{\mathrm{w}}=0,92$ bei $S$. aureus eine geringfügige Wachstumsverlangsamung; unterhalb des angeführten Wertes nimmt die Hemmwirkung zu, und unterhalb von $\mathrm{a}_{\mathrm{w}}=0,90$ ist bereits eine deutliche Hemmung festzustellen (GENIGEORGIS u.a. 1971, Troller und Stinson 1975). Als Mindestwerte für das Wachstum werden 0,83 (HILL 1973) und 0,86 (ScotT 1957) angegeben. Enterotoxin bildet sich bei $a_{w}$-Werten über 0,93 (Troller und Stinson 1975). Wenn die Vermehrungsgeschwindigkeit von $S$. aureus durch $\mathrm{pH}$-Senkung auch vermindert wird, so gedeiht er doch in einem breiten Bereich bis $\mathrm{pH} \mathrm{4,0}$ und produziert Toxine (Review: Riemann u.a. 1972). Anaerobe Verhältnisse bewirken eine Verzögerung sowohl des Wachstums (GENigeorgis u.a. 1971, Buchanan und Sohlberg 1972) als auch der Toxinbildung (BARber und Deibel 1972) von $S$. aureus. Für das Wachstum dieses Organismus ist eine Mindesttemperatur von $6,7^{\circ} \mathrm{C}$ erforderlich; zur Toxinbildung kann es unter anaeroben Verhältnissen noch bei $10^{\circ} \mathrm{C}$ kommen. Mit steigender Temperatur nehmen Wachstum und Toxinbildung an Geschwindigkeit zu und kommen dann bei $45^{\circ}-46^{\circ} \mathrm{C}$ zum Erliegen (Review: Riemann u.a. 1972). Zur Toxinbildung sind $1-4 \cdot 10^{7}$ Keime/g erforderlich. In der Oberflächenschicht der Wurst werden koagulasepositive Staphylokokken oft in Mengen von $10^{4}-10^{6} \mathrm{Keimen} / \mathrm{g}$ festgestellt, im Kern hingegen gewöhnlich in Mengen von weniger als 100 Keimen/g (BARBER und DeIBel 1972).

Nitrit hemmt das Wachstum von $S$. aureus unter aeroben Verhältnissen nur geringfügig, unter anaeroben Verhältnissen im sauren $\mathrm{pH}-$ Bereich hingegen sehr stark (Castellani und Niven 1955, Buchanan and Sohlberg 1972). Nitrat wird erst nach seiner Reduktion zu Nitrit (Lechowich u.a. 1956) oder in Verbindung mit letzterem (JEDLICKA u.a. 1975) wirksam.

McLean u.a. (1968) fanden, dass bei Ansteigen des Salzgehalts die Bildung von Staphylokokken-Enterotoxin B stärker als das Wachstum des Organismus gehemmt wird. Eine starke Minderung des Toxinbildungsvermögens trat ab einem Salzgehalt von $2 \%$ ein. Bei Zusatz von Nitrit und Senkung der Temperatur von $37^{\circ} \mathrm{C}$ auf $20^{\circ} \mathrm{C}$ zeigte sich lediglich eine additive Hemmwirkung dieser beiden Faktoren. Nitrit allein hatte keine grosse Wirkung.

Wachstum und Toxinbildung der Staphylokokken in der Rohwurst werden somit im wesentlichen von anderen Faktoren als vom Nitrit oder Nitrat bestimmt (Bild 4) (Riemann u.a. 1972). Jedoch Jedlicka u.a. (1975) fanden, 
dass bei Berücksichtigung sämtlicher Endprodukt-Eigenschaften $100 \mathrm{ppm}$ $\mathrm{NaNO}_{3}$ und 75 ppm $\mathrm{NaNO}_{2}$ die optimale Kombination im Hinblick auf die Hemmung der Staphylokokken darstellen. Die inhibitorische Wirkung des Nitrits schien insbesondere unter anaeroben Verhältnissen eher additiver Natur zu sein. Der Einfluss der Nitritkonzentration auf die Toxinbildung wurde von ihnen nicht untersucht.

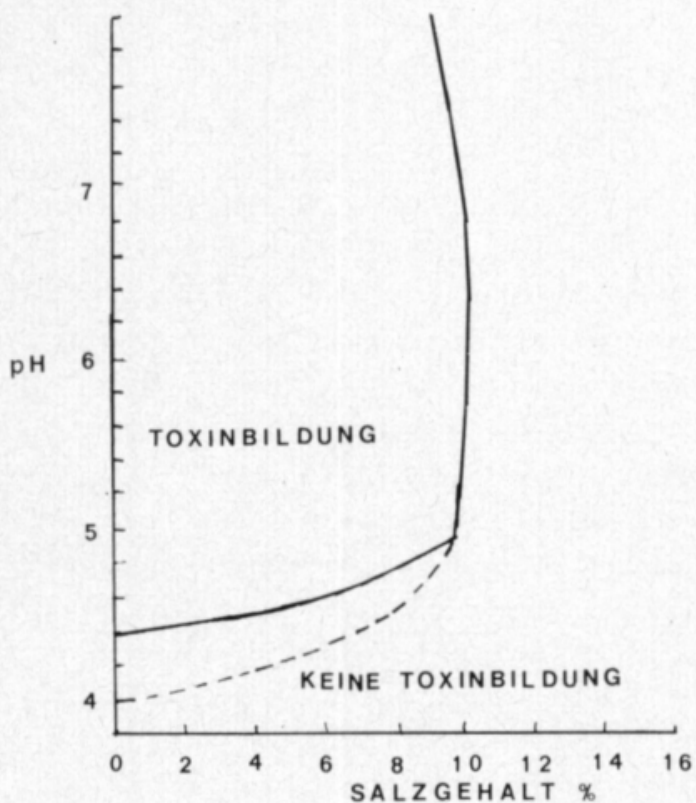

Bild 4. pH-Wert-Salz-Kombinationen, bei denen auf künstlichem Nährboden eine aerobe Bildung (oberhalb der Kurven) oder keine aerobe Bildung (unterhalb der Kurven) von StaphylokokkenEnterotoxin A, B und C stattfand. Beimpfung mit $10^{7}$ Keimen/g oder $10^{8}$ Keimen/g (- - - ) ; (Review: RIEMANN u.a. 1972).

Barber und Deibel (1972) beobachteten bei Verwendung von Starterkulturen $^{1}$ ) ein Wachstum von Staphylokokken auf der Wurstoberfläche, auf der aerobe Verhältnisse vorliegen. Unter anaeroben Bedingungen wurde nur eine schwache oder überhaupt keine Toxinbildung festgestellt. Durch 1,5\% GdL wurde das Wachstum der Staphylokokken sowohl unter aeroben als auch unter anaeroben Verhältnissen verhindert; Wachstum fand unter aeroben Verhältnissen bei 1,3\% GdL, unter anaeroben Verhältnissen bei 1,0\% GdL statt. Unterhalb von $\mathrm{pH}$ 5,7 vermochten die meisten Stämme bei anaeroben Verhältnissen kein Toxin zu bilden; unter aeroben Verhältnissen lag diese Grenze dagegen bei pH 5,1.

DALY u.a. (1973) fanden, dass unter simulierten Rohwurstverhältnissen auch Starterkulturen ${ }^{2}$ ) das Staphylokokken-Wachstum stark hemmen. In ihren Versuchen kam es, wurden keine Starterkulturen zugesetzt, in den ersten 50 Stunden zu einer sehr heftigen Vermehrung der Staphylokokken. Laktobazillen allein hatten eine geringere Hemmwirkung als ein Laktobazillen-

1) Pediococcus cerevisiae.

${ }^{2}$ ) Pediococcus cerevisiae (Lactacel), Pediococous cerevisiae + L. plantarum (Lactacel MC) und Lactobacillus plantarum (Lactacel DS). 
Pediokokken-Gemisch. Chemisches Säuern (Einsatz von GdL) allein bewirkte keine wesentliche Hemmung des Staphylokokken-Wachstums, ergab aber kombiniert mit Starterkulturen einen starken Effekt. Bei hohen Temperaturen $\left(37^{\circ} \mathrm{C}\right.$ und $\left.30^{\circ} \mathrm{C}\right)$ wurde die Säurebildung der Starterkulturen beschleunigt; die Hemmung der Staphylokokken war entsprechend intensiver als bei niedriger Temperatur $\left(21^{\circ} \mathrm{C}\right)$. Daly u.a. arbeiteten in ihren Versuchen mit einer ziemlich niedrigen Nitratmenge (100 ppm), was vielleicht einer der Gründe für das schnelle Wachstum der Staphylokokken in den Kontrollwürsten war.

Niskanen und Nurmi (1976) konstatierten, dass bei typisch finnischer Rohwurst, deren Reifetemperatur anfangs höchstens $24^{\circ} \mathrm{C}$ beträgt und innerhalb von einigen Tagen auf etwa $20^{\circ} \mathrm{C}$ gesenkt wird, eine hohe Anzahl Staphylococcus aureus nur in den allerseltensten Fällen möglich ist. Sie sind der Auffassung, dass der $\mathrm{pH}$ durch Einsatz von Starterkulturen ${ }^{\mathbf{1}}$ ) oder GdL gesenkt werden müsse, um das diesbezügliche Risiko so klein wie möglich zu halten. Bei starker Kontamination mit $S$. aureus $\left(10^{6} \mathrm{Keime} / \mathrm{g}\right)$ kam es ohne Starterkultur-Zusatz zur Bildung von Enterotoxin A, nicht aber bei Einsatz von Starterkultur. Bei sehr starker Staphylokokken-Kontamination bildete sich innerhalb der ersten drei Tage selbst bei Einsatz von Mikrokokken-Starterkultur Enterotoxin C, das allerdings am siebten Tag nicht mehr nachzuweisen war. Zur Bildung von Enterotoxin B kam es hingegen nicht. Durch Mikrokokken-Starterkultur wurde die Toxinbildung stets dann verhindert, wenn mehr Mikrokokken als Staphylokokken vorhanden waren. Durch LaktobazillenStarterkultur wurde das Wachstum der Staphylokokken durchweg gehemmt.

TATini u.a. (1976) haben ermittelt, dass durch Räuchern das Wachstum der Staphylokokken in Rohwurst (Pepperoni) gehemmt wird. Allerdings kam es in ihren Versuchen selbst bei der Kontrollwurst mit einem Staphylokokkengehalt von $4 \cdot 10^{7}$ Keimen $/ g$ nicht zur Bildung von Enterotoxin. Nach Tatini und seinen Mitarbeitern birgt Wurst vom Pepperoni-Typ, was die Staphylokokken betrifft, kein gesundheitliches Risiko in sich.

LABots (1977) fand, dass durch Erhöhung des Nitritzusatzes von 0 auf 120 ppm bei nassgepökelter Rohwurst das Wachstum von S. aureus gehemmt wurde. Ohne Nitritzugabe nahm dieser Organismus in der Oberflächenschicht von $10^{3}$ auf $10^{6}$, im Kern auf $10^{4} \mathrm{Keime} / \mathrm{g}$ zu. Bei den niedrigeren Nitritzusätzen (bis $60 \mathrm{ppm}$ ) vermehrte sich $S$. aureus anfänglich, begann aber dann abzusterben. Das Absterben setzte mit steigender Nitritkonzentration früher ein. Bei Zusatz von $120 \mathrm{ppm} \mathrm{NaNO}_{2}$ fand überhaupt keine Vermehrung mehr statt. Bei sämtlichen Nitritkonzentrationen lag der Gehalt an S. aureus im Kern der Wurst wesentlich niedriger als in der Oberflächenschicht.

In entsprechenden luftgetrockneten, ungeräucherten Rohwürsten nahm $S$. aureus an der Oberfläche zahlenmässig stark zu, und der Nitritzusatz $\left(30-180\right.$ ppm $\left.\mathrm{NaNO}_{2}\right)$ hatte keinen Einfluss auf das Wachstum der Staphylokokken. Im Kern der Wurst lag die $S$. aureus-Konzentration beträchtlich niedriger, eine entscheidende Wirkung hatte der Nitritgehalt aber auch hier nicht. Der pH-Wert zeigte deutliche Auswirkungen auf das Wachstum von

1) Lactobacillus plantarum und Micrococcus sp. (Duploferment 66). 
S. aureus. Bei Verringerung des Nitritzusatzes von $60 \mathrm{ppm}$ auf $30 \mathrm{ppm}$ nahm der $\mathrm{pH}$ um so viel ab, dass bei der niedrigeren Nitritkonzentration ein schnelleres Absterben von $S$. aureus stattfand, dem allerdings eine deutliche Vermehrungsphase voranging. (LABots 1977).

\section{Clostridium botulinum}

Riemann u.a. (1972) äussern in ihrem umfangreichen Aufsatz die Vermutung, dass in den USA Clostridium botulinum gelegentlich in FleischwarenRohmaterial auftritt. Nach Roberts und Ingram (1977) enthält in England das Schweinefleisch im Durchschnitt 2 Keime/kg C. botulinum. Da die Klostridien mit Ausnahme hohen Redox-Potentials alle beim Herstellungsprozess auftretenden Verhältnisse ausgezeichnet vertragen, ist auch bei der Produktion von Rohwurst Sorge dafür zu tragen, dass es nicht zu einer Vermehrung und Toxinbildung von $C$. botulinum kommt.

Nach NinnivaARA (1955) haben die Lebensfunktionen der in der Wurst enthaltenen Bakterien die Tendenz, das Redox-Potential in den für die Vermehrung der anaeroben Bakterien günstigen Bereich herabzusetzen. Nitratzusatz stabilisiert in gewissem Masse diese Veränderungen. Die Verwendung von Starterkulturen führt zur Reduktion des Nitrats zu Nitrit und weiter zu Stickstoffoxid. Besonders diese Reaktionen erhöhen das Redox-Potential und stabilisieren weitgehend seine Veränderungen. Dies hat zur Folge, dass die Tätigkeit der anaeroben Bakterien in der Wurst geschwächt wird.

Die proteolytischen Stämme zeigen unterhalb von $10^{\circ}-12^{\circ} \mathrm{C}$ kein Wachstum mehr; einige nichtproteolytische Stämme gedeihen jedoch sogar noch bei $3^{\circ} \mathrm{C}$. Die Toxinbildung kommt im allgemeinen eher als das Wachstum zum Stillstand. C. botulinum gedeiht bei ziemlich niedrigen $\mathrm{pH}$-Werten $(\mathbf{4 , 8}-\mathbf{5}, \mathbf{0})$ (RIEMANN u.a. 1972). Mit sinkender Temperatur steigt jedoch der für das Wachstum der sechs Stämme von $C$. botulinum erforderliche Mindest-pH; bei $29^{\circ} \mathrm{C}$ beträgt er $5,0-5,4$, bei $16^{\circ} \mathrm{C} 5,4$, bei $8^{\circ} \mathrm{C} 5,7-5,9$ und bei $5^{\circ} \mathrm{C} 6,2$ (Emodi und Lechожісн 1969). Die Ergebnisse sind von Stamm zu Stamm verschieden. Bei Laborversuchen hatte der Salzgehalt keinen Einfluss auf den für das Wachstum erforderlichen Mindest-pH (Review: Rremann u.a. 1972). Bild 5 zeigt das Wachstum von $C$. botulinum in Abhängigkeit vom $\mathrm{pH}$ und vom Salzgehalt. Der Nitritgehalt blieb unberücksichtigt.

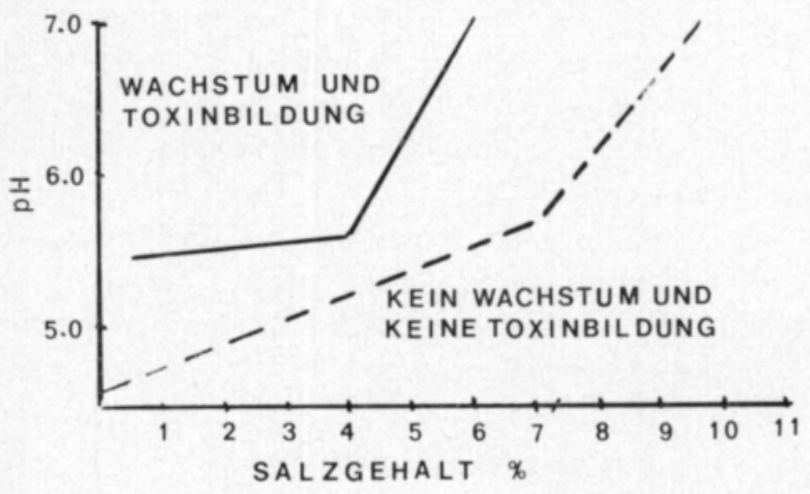

Bild 5. pH-Wert-Salz-Kombinationen, bei denen Wachstum und Toxinbildung (oberhlb der Kurven) bzw. kein Wachstum und keine Toxinbildung (unterhalb der Kurven) stattfand. Clostridium botulinum Typ A und B (—) sowie Typ E (-...-) (RIEMANN u.a 1972). 
Bei einem Salzgehalt der Wasserphasen von 6,3-9,0\% wurde Toxinbildung beobachtet (Review: RiEmann u.a. 1972). Mit steigendem Salzgehalt, sinkendem $\mathrm{pH}$, steigendem Nitritgehalt und abnehmender Temperatur wird das Wachstum von $C$. botulinum gehemmt; in der Rohwurst ist die Gesamtwirkung dieser Faktoren so beträchtlich, dass eine Vermehrung von $C$. botulinum im Bereich des Unwahrscheinlichen liegt (Review: Riemann u.a. 1972, Roberts und Ingram 1977). Aus diesem Grunde wurde die durch Rohwurst gegebene Botulismus-Gefahr bisher auch nur sehr wenig untersucht. Christiansen u.a. (1975) haben hingegen eine sehr ausgedehnte Untersuchung über die Toxinbildungsfähigkeit von $C$. botulinum unter verschiedenartigen Bedingungen durchgeführt. Als Versuchswurst diente Summer Sausage, die bei $32^{\circ} \mathrm{C}$ mit oder ohne Starterkulturen ${ }^{1}$ ) fermentiert wurde. Die Fermentation wurde durch Erhitzen auf $58,3^{\circ} \mathrm{C}$ abgebrochen, jedoch können aus den Ergebnissen Schlüsse gezogen werden, die auch hinsichtlich anderer Rohwursttypen Gültigkeit haben dürften. Die Toxinbildung wurde durch Lagerung der Würste bei $27^{\circ} \mathrm{C}$ verfolgt.

Die Bedeutung des pH-Wertes trat in sämtlichen Stadien des Versuchs sehr deutlich in Erscheinung. Durch Zugabe von Glukose wurde die Möglichkeit für eine $\mathrm{pH}$-Abnahme geschaffen, und bei Zusatz von Starterkultur sank der $\mathrm{pH}$ rasch auf einen ausreichend niedrigen Wert. Nitrat verhinderte die Toxinbildung, sofern es rechtzeitig vor dem $\mathrm{pH}$-Abfall zu Nitrit reduziert wurde. Bei Wurst mit Glukosezusatz genügten 50 ppm $\mathrm{NaNO}_{2}$ um die Toxinbildung zu unterbinden, bei glukosefreier Wurst waren hierzu 150 ppm erforderlich. Zahlenmässig blieb $C$. botulinum während der gesamten Versuchsdauer (112 Tage) und bei sämtlichen erprobten Zusatzstoff-Kombinationen praktisch konstant, so dass also nicht die Bakterienzahl sondern die Toxinbildung der ausschlaggebende Faktor ist (Christiansen u.a. 1975).

Mit Toxinbildung in kontaminierter Wurst ist also dann zu rechnen, wenn weder Nitrit noch Glukose noch Starterkultur noch GdL zugesetzt wird eine in der Praxis undenkbare Situation. Von einer nennenswerten BotulismusGefahr kann bei Rohwurst deshalb nicht die Rede sein.

\section{Clostridium perfringens}

Zyss u.a. (1967) konstatierten, dass Clostridium perfringens den RohwurstHerstellungsprozess u.U. überstand, jedoch innerhalb der ersten drei Tage zahlenmässig stark zurückging. Die Absterbequote nahm mit steigender Temperatur zu. Durch Erhöhung des Salz- und Nitritgehalts wurde zwar das Wachstum dieses Organismus gehemmt, kam aber in Laborversuchen auch bei $8 \% \mathrm{NaCl}$ und $200 \mathrm{ppm} \mathrm{NaNO}_{2}$ noch nicht völlig zum Erliegen. Andere Arbeiten über das Wachstum von $C$. perfringens unter Rohwurst-Verhältnissen liegen kaum vor, und aus den mit rohem Fleisch oder erhitzten Produkten durchgeführten Versuchen können keine Schlüsse bezüglich Rohwurst gezogen werden. Auf Rohwurst zurückzuführende $C$. perfringens-Nahrungsmittelvergiftungen werden nur selten festgestellt. RoBERTs und INGRAM (1977) haben ausgeführt, dass zwar verschiedene Klostridien in nicht wärmebehandeltem

1) Lactobacillus plantarum und Pediococcus cerevisiae (Lactacel MC). 
Schinkenspeck unter gewissen Verhältnissen gediehen, dass aber $C$. perfringens in keinem einzigen Falle Wachstum zeigte.

\section{Bacillus cereus}

Nach Raevuori (Review 1976) wurde Bacillus cereus im Rahmen mehrerer verschiedener Forschungsarbeiten in $2-77 \%$ der insgesamt untersuchten Lebensmittelproben und in $\mathbf{2 6 - 5 8} \%$ der untersuchten Fleisch- und Fleischwarenproben festgestellt. In finnischer Rohwurst ist B. cereus nur äusserst selten und auch dann nur in geringer Zahl anzutreffen (РонJA 1976, mündliche Information). Als Mindestwachstumsbedingungen fordert dieser Organismus $\mathrm{pH} 4,5,10^{\circ} \mathrm{C}$ und $\mathrm{a}_{\mathrm{w}} 0,95$. ÖSTLUND und REGNER (1968) konstatierten, dass $B$. cereus sich in der Rohwurst gut vermehren kann. In ihrer Untersuchung ging der Gehalt an B. cereus jedoch sehr schnell wieder zurück. Bei Abweichung mehrerer Faktoren vom Optimum und Anwesenheit von Nitrit und eventuell Erythorbat ist sein Wachstum weitgehend gehemmt. РонJA und NinnvaAra (1957) haben auch festgestellt, dass Micrococcus $M_{53}$ eine antagonistische Wirkung auf die den Familien Achromobacteriaceae, Enterobacteriaceae und Bacillaceae angehörenden Arten ausübt. $B$. cereus wurde auch schwach gehemmt. $B$. cereus-Nahrungsmittelvergiftung infolge Rohwurstverzehrs ist deshalb höchst unwahrscheinlich, und bisher sind auch keine derartigen Fälle bekannt (Review: RAEVUORI 1976).

\section{4 Nitrosamine in der Rohwurst}

Auch die Rohwurst hat man in die Nitrosamin-Forschung einbezogen. Mit Ausnahme des Summer-Sausage-Typs wurde Rohwurst bei der Herstellung nicht erhitzt. Wärmebehandlung, insbesondere Braten, hat ein Ansteigen der Nitrosaminbildung zur Folge. Allerdings ist der niedrige $\mathrm{pH}$ der Rohwurst gerade der Bildung stark karzinogener Nitrosamine förderlich. Auch bei Zimmertemperatur und darunter können sich Nitrosamine bilden (Review: Crosby und SAWYER 1976).

In Tabelle 2 sind die von verschiedenen Forschern in einigen Rohwurstsorten festgestellten Nitrosaminmengen zusammengestellt. Die Angaben wurden unabhängig von der Art des jeweiligen Nitrosamins in ppb gemacht. Zur Vermittlung eines eindeutigen Bildes wäre eine umfangreiche Tabelle erforderlich, wobei die durch die Analysensysteme bedingten Unterschiede trotzdem bestehenbleiben würden. Die Analysenverfahren sind noch mit beträchtlichen Unsicherheitsfaktoren behaftet, wodurch die generelle Erfassung des NitrosaminProblems sehr erschwert wird (Proc. ISNMP 2. 1977).

Die Geschwindigkeit der Umsetzung zwischen Nitrit und Aminen ist vom $\mathrm{pH}$ abhängig. Es handelt sich dabei um folgende Reaktionen (Gleichungen 5-8):

$$
\begin{aligned}
& 2 \mathrm{HNO}_{2} \leftrightarrows \mathrm{N}_{2} \mathrm{O}_{3}+\mathrm{H}_{2} \mathrm{O} \\
& \mathrm{R}_{2} \mathrm{NH}+\mathrm{N}_{2} \mathrm{O}_{3} \rightleftarrows \mathrm{R}_{2} \mathrm{~N} \cdot \mathrm{NO}+\mathrm{HNO}_{2} \\
& \text { Geschwindigkeit }=\mathrm{k}_{1}\left[\mathrm{R}_{2} \mathrm{NH}\right]\left[\mathrm{HNO}_{2}\right]^{2} \\
& \text { Geschwindigkeit }=\mathrm{k}_{2}[\text { Amin }][\text { Nitrit }]^{2}
\end{aligned}
$$


Tabelle 2. Auftreten von Nitrosaminen in der Rohwurst.

\begin{tabular}{|c|c|c|c|}
\hline Wursttyp & Nitrosamin (ppb) & Analysenverfahren & Forscher \\
\hline Pepperoni $\left.(\mathbf{1})^{\mathbf{1}}\right)$ & 3 & GC & Fazio et al. 1971 \\
\hline Salami & $1-2$ & , & b \\
\hline Thüringer (2) & 2 & , & - \\
\hline Salami $\quad(6)$ & $\left.\left.5^{1}\right): \mathrm{NB}^{2}\right), 1: 1-4$ & $\mathrm{GC}+\mathrm{MS}$ & Grosby et al. 1972 \\
\hline Salami $\left.\quad()^{3}\right)$ & $2: 20-80$ & $\mathrm{GC}+(\mathrm{MS})$ & Sen 1972 \\
\hline Dry sausage ()$\left.^{3}\right)$ & $3: 10-20$ & , & 3 \\
\hline Salami $\quad(12)$ & $7: N B, 5: 3-5$ & GC & Panalaks et al. 1973 \\
\hline Salami (4) & NB & $\mathrm{GC}+(\mathrm{MS})$ & Panalaks et al. 1974 \\
\hline Mettwurst (21) & 12: NB, 9:2-112 & $\mathrm{GC}+(\mathrm{MS})$ & Panalaks et al. 1974 \\
\hline
\end{tabular}

$\begin{array}{lll}\mathrm{NB}=\text { negativer Befund } & \text { 1) } & \text { Anzahl der Proben } \\ \mathrm{GC}=\text { Gaschromatographie } & \text { 2) } & \text { Nitrosamingehalt } \\ \text { MS = Massenspektrometrie } & \text { 3) } & \text { Nicht angeben }\end{array}$

Die Geschwindigkeitskonstante $\mathrm{k}_{1}$ in Gleichung (7) ist vom pH-Wert unabhängig; schreibt man jedoch die Gleichung (7) in der Form (8), so hat der $\mathrm{pH}$-Wert Einfluss auf den Betrag der Geschwindigkeitskonstante $\mathrm{k}_{2}$. Das Amin reagiert bei dieser Umsetzung nur in freier Form. Der pH-Einfluss im $\mathrm{pH}$-Bereich $\mathbf{9 - 5}$ ist in der Form definiert, dass bei Rückgang des $\mathrm{pH}$ Wertes das nichtionisierte Amin mengenmässig auf ein Zehntel sinkt und die salpetrige Säure um das Zehnfache zunimmt. Da in Gleichung (7) die Konzentration der salpetrigen Säure im Quadrat erscheint, ergibt sich als Nettoeffekt ein Anstieg der Reaktionsgeschwindigkeitskonstante auf das Zehnfache. Im $\mathrm{pH}$-Bereich nahe dem $\mathrm{pK}$ der salpetrigen Säuren $(\mathrm{pH}=3,4)$ liegt praktisch schon das gesamte Nitrit als salpetrige Säure vor; der Einfluss des $\mathrm{pH}-$ Wertes auf die Reaktionsgeschwindigkeitskonstante basiert im wesentlichen nur auf der mengenmässigen Abnahme von $\mathrm{R}_{2} \mathrm{NH}$ (Review: MirvisH 1975, Crosby und SAwyer 1976). Die Amine werden um so leichter nitrosiert je weniger basisch sie sind. Die Bildung von Nitrosaminen kann auch enzymatisch erfolgen, wobei sich dann die Kinetik von Fall zu Fall ändert; die Reaktionsgeschwindigkeit ist hierbei jedoch im allgemeinen direkt proportional zur Nitritkonzentration (Review: MrRvish 1975). Desgleichen werden die langkettigen und aromatischen Amine leichter nitrosiert als die hydrophilen - und stärker karzinogenen - Amine. N-Nitrosoverbindungen können sich noch im Verdauungsapparat aus freiem Nitrit und Aminen bilden, wobei dann auch die im Magen stattfindende bakterielle Nitratreduktion (SANDER und SEIF 1969) sowie die im Speichel erfolgende Reduktion von Nitrat in Nitrit (Tannenbaum u.a. 1974) mit ins Spiel kommen. Die Nitrosamine sind dabei keinesfalls die einzigen alkylierenden Nitrit-Umsetzungsprodukte, sondern Nitrit vermag auch mit zahlreichen anderen Verbindungen zu reagieren. Es wäre wohl richtiger, von karzinogenen N-Nitrosoverbindungen allgemein zu sprechen, aber die Forschung war bisher hauptsächlich auf die Nitrosamine konzentriert (Proc. ISNMP 2. 1977). Die mit diesem Sachkomplex verbundenen chemischen, analytischen und toxikologischen Betrachtungen gehen allerdings schon über den Rahmen dieser Arbeit hinaus. 
Unter Berücksichtigung der obigen Ausführungen besteht also wirklich aller Grund, den Nitritzusatz der Rohwurst zu senken, da der $\mathrm{pH}$-Wert nach der Fermentation bzw. dem chemischen Säuern abgenommen hat und die Wurst sekundäre Amine enthält.

Kueper und Trelease (1974) haben eine Zusammenfassung über eine grossangelegte Untersuchung betreffend den Einsatz von Nitrit und Nitrat mit oder ohne Starterkulturen ${ }^{1}$ ) bei der Rohwurstfabrikation veröffentlicht. In dieser Untersuchung wurden bei keiner einzigen Versuchswurst des ThüringerTyps Nitrosamine festgestellt. Die Versuchsbedingungen waren folgende:

1) $0-150 \mathrm{ppm} \mathrm{NaNO}_{2}$

2) $0-1500 \mathrm{ppm} \mathrm{KNO}_{3}$

3) Fermentationstemperaturen: $18^{\circ} \mathrm{C}$ und $40^{\circ} \mathrm{C}$

4) Lagertemperaturen: $-18^{\circ} \mathrm{C}, 15^{\circ} \mathrm{C}$ und $34^{\circ} \mathrm{C}$

5) Lagerzeit: $0-8$ Wochen

6) In Scheiben geschnitten, gebraten

Nagata und Mirna (1974) haben eine gründliche Untersuchung über den Nitrosamingehalt in der Bundesrepublik Deutschland hergestellter Fleischwaren angestellt. Sie ermittelten, dass Salami bei Einsatz von Nitrat einen etwas geringeren Nitrosamingehalt aufwies als beim Arbeiten mit einer NitratNitrit-Salz-Mischung. Der Unterschied war jedoch unbedeutend. Durch Zusatz von Mikrokokken-Starterkultur hingegen konnte der Nitrosamingehalt deutlich gesenkt werden (Tabelle 3). Der Einsatz von Askorbinverbindungen bei der Rohwurstherstellung führte jedoch zu widersprüchlichen Ergebnissen: Die Wirkung war von der Konzentration des Nitrits und der Askorbinverbindung abhängig und hatte für die einzelnen Nitrosamine und in den einzelnen Versuchen unterschiedliche Richtung. In den meisten Fällen führten die Askorbinverbindungen (100-1000 ppm) allerdings zu einem Anstieg des Nitrosamingehalts, und nach Nagata und Mirna würde erst eine Konzentration $>1500$ ppm eine senkende Wirkung haben. Die Wirkung des GdL war ebenso indifferent. Die Nitrosopyrrolidin-Menge stieg bei Zusatz von GdL an, während die übrigen untersuchten Nitrosamine (Tabelle 3) mengenmässig zurückgingen. Allerdings arbeiteten die beiden Forscher nur mit 1/10 des bei Rohwurst üblichen GdL-Zusatzes, jedoch mit normaler Nitritmenge.

Nagata und Mirna konstatieren, dass durch Senkung des Nitritgehaltes und Einsatz anderer Zusatzstoffe keine völlig nitrosaminfreien Fleischwaren erzielt werden, empfehlen aber trotzdem das Arbeiten mit verringerten Nitritund Nitratzusätzen. Gleichzeitig weisen sie darauf hin, dass es vorteilhaft ist, ein Nitrit-Nitrat-Gemisch (2:1) zuzusetzen, da im Fleisch ja auf jeden Fall ein Teil des Nitrits in Nitrat umgewandelt und zu Beginn durch die Anwesenheit von Nitrat das Gleichgewicht in Richtung Nitrit verschoben wird.

Palumbo u.a. (1974) untersuchten im industriellen und im halbtechnischen Massstab ohne oder mit Starterkulturen ${ }^{2}$ ) hergestellte Lebanon-bologna-Würste auf sechs verschiedene Nitrosamine. Keine einzige Probe ergab positiven

1) Lactobacillus plantarum und Pediococcus cerevisiae.

2) Lactobacillus plantarum und Pediococcus cerevisiae (Lactacel MC). 
Tabelle 3. Einfluss der Lagerzeit und des Zusatzes von Nitrat und Baktofermenten bzw. von Nitrit-Nitrat-Gemischen auf den Gehalt an Nitrosaminen in Teewurst $(2.6 \% \mathrm{NaCl})$ (NAGATA und Mirna 1974).

\begin{tabular}{|c|c|c|c|c|c|c|c|}
\hline $\begin{array}{c}\text { Lagerzeit } \\
\text { (Tage) }\end{array}$ & Zusatz von & $\begin{array}{r}\mathrm{ppm} \\
\mathrm{NaNO}_{2} \\
\end{array}$ & $\begin{array}{l}\text { Rest - } \\
\mathrm{KNO}_{3}\end{array}$ & DMNA & $\begin{array}{r}\mathrm{p}_{1} \\
\mathrm{DA} \mathrm{NA}\end{array}$ & NPIP & P NPYR \\
\hline \multirow{4}{*}{14} & $\begin{array}{l}600 \mathrm{ppm} \mathrm{KNO}_{3} \\
\text { Baktoferment } \\
65 \text { ppm NaNO}\end{array}$ & 72 & 101 & $<2$ & $<2$ & $<2$ & 5 \\
\hline & $\begin{array}{l}50 \mathrm{ppm} \mathrm{KNO} \mathrm{KNO}_{3} \\
600 \mathrm{ppm} \mathrm{KNO}\end{array}$ & 14 & 10 & 3 & $<2$ & $<2$ & 15 \\
\hline & $\begin{array}{l}\text { Baktoferment } \\
65 \text { ppm } \mathrm{NaNO}_{2}\end{array}$ & 30 & 94 & $<2$ & $<2$ & $<2$ & $<2$ \\
\hline & $50 \mathrm{ppm} \mathrm{KNO}_{3}$ & 8 & 11 & 17 & $<2$ & $<2$ & 22 \\
\hline
\end{tabular}

Befund, was nach Ansicht der Forscher auf den verschwindend geringen Gehalt des Fleisches an sekundären Aminen zurückzuführen war, wodurch die Nitrosaminbildung verhindert wurde.

Verschiedene Bakterien fördern die Nitrosaminbildung, und zwar durch Senkung des $\mathrm{pH}$-Werts und vorläufig noch ungeklärte biologische Aktivierung der Nitrosamin-Synthese (SANDER 1968, SANDER und Schweisberg 1972 , Collins-Thompson u.a. 1972). Auch im Speichel erfolgt eine Umwandlung von Nitrat in Nitrit (TAnnenbaum u.a. 1974), wodurch die Nitrosamin-Synthese im Magen bei niedrigem $\mathrm{pH}$ ermöglicht wird (SANDER u.a. 1968, LANE u.a. 1974). Auch diese Faktoren sind bei der Einschätzung des durch Rohwurst gegebenen Gesamtrisikos in Rechnung zu stellen (NAGATA und Mirna 1974).

Obgleich erwiesenermassen gewisse Bakterien die Nitrosamin-Synthese fördern, haben PÁumbo u.a. (1974) in ihrer Untersuchung auch in den Proben keine Nitrosamine festgestellt, die unter Einsatz von Starterkulturen hergestellt worden waren. Möhler und Hallenmeyer (1974) haben keine im Zusammenhang mit der von Bacillus cereus oder von Mikrokokken bewirkten Nitratreduktion auftretende bakterielle Nitrosaminbildung beobachtet. Freilich sind nicht alle Bakterien der Nitrosamin-Synthese förderlich (CoLLINs-THompson u.a. 1972), und durch Einsatz von Starterkulturen lässt sich der Nitrosamingehalt sogar senken (Tabelle 3, Nagata und Mirna 1974).

Das Vorkommen von N-Nitrosoverbindungen ist eine äusserst schwierig zu klärende Angelegenheit. Infolge der komplizierten Analytik haben, wie aus obigem Text hervorgeht, die einzelnen Forscher deutlich voneinander abweichende Ergebnisse erhalten, wobei allerdings nicht vergessen werden darf, dass sich ihre Untersuchungen auch hinsichtlich der Zusatzstoffmengen, der Fleischwarenart, der Verfahrenstechnik, der Rohstoffe und der mikrobiologischen Faktoren unterschieden. Trotzdem kann auch unter Berücksichtigung all dieser Umstände konstatiert werden, dass die Rohwurst bezüglich $\mathrm{N}$-Nitrosoverbindungen etwa der gleichen Gefahr wie die übrigen Fleischwaren ausgesetzt ist. Ein wie grosses Risiko diese Verbindungen bei Fleischwaren allgemein genommen darstellen, harrt noch der Klärung. 


\section{Eigene Untersuchungen}

\section{1 Zweck der Untersuchungen}

Mit der vorliegenden Untersuchung wurde bezweckt, die Nitrit- und NitratMindestkonzentrationen zu ermitteln, die ausreichen, um den heutigen hygienischen und technischen Anforderungen entsprechende Rohwurst herzustellen. Ausgehend von dieser Zielsetzung wurde der Einfluss von Starterkulturen, verschiedenen Zusatzstoffen und unterschiedlichen Verfahrenstechniken auf die Restnitrit- und -nitratmengen sowie auf die Eigenschaften der Rohwurst bei verschieden grossen Nitrit- und/oder Nitratzusätzen untersucht.

\section{2 Versuchsanordnungen}

Die Untersuchung gliederte sich in drei Teile:

1) Präliminarversuche, in denen der Einfluss verschiedener Faktoren auf den Nitrit- und Nitratgehalt sowie auf die Eigenschaften der Rohwurst geklärt wurde (Versuchsreihen I-XVI).

2) Vergleichende Versuche, in denen die Wirkung auf Grund von Teil 1) gewählter verminderter Nitrit- und Nitratzusätze an der Wirkung der heute in der Industrie allgemein üblichen Nitrit- und Nitratzusätze verglichen wurde (Versuchsreihen VI-V6).

3) Salmonellenversuche, in denen das Wachstum von Salmonellen bei den im Teil 2) verwendeten verschiedenen Nitrit- und Nitratgehalten verglichen wurde (Versuchsreihen S1-S2).

Der Zweck der Präliminarversuche, die in ihnen verwendeten Zusatzmittelmengen sowie Starterkulturen betreffende Angaben sind in Tabelle 4 zusammengestellt. In die Spalte "Bemerkungen" sind eventuelle Abweichungen vom Grundherstellungsverfahren eingetragen (siehe Abschnitt 3. 3. 1). Sämtliche Präliminarversuche wurden komplett zweimal durchgeführt, so dass sich in diesem Teil der Untersuchung insgesamt 168 Versuchsposten ergaben (Tabelle 4).

Die in den vergleichenden und in den Salmonellenversuchen verwendeten Zusatzmittelmengen sowie Starterkulturen betreffende Angaben sind in Tabelle 5 zusammengestellt.

Statt Wurstposten $\mathrm{x}$ wird im Text die Bezeichnung Wurst $\mathrm{x}$ verwendet.

Die Würste wurden im Institut für Fleischtechnologie der Universität Helsinki hergestellt. Die Herstellung der Wurstmassen für die Salmonellenversuche erfolgte im Institut für Fleischtechnologie, die Zugabe der Salmonellen und das Reifen der Würste in der Versuchsfabrik des Instituts für Lebensmittelhygiene der Veterinärmedizinischen Hochschule. Die mit den Salmonellenversuchen verbundenen Bestimmungen wurden beim Staatlichen Veterinärmedizinischen Institut in Auftrag gegeben. 
Tabelle 4. Versuchsanordnungen der Präliminarversuche, Versuchsreihen I-XV1.

\begin{tabular}{|c|c|c|c|c|c|c|c|c|c|}
\hline Versuch & $\begin{array}{l}\text { Zweck des } \\
\text { Versuchs }\end{array}$ & $\begin{array}{l}\text { Wurst- } \\
\text { posten }\end{array}$ & $\begin{array}{c}\mathrm{NaNO}_{2} \\
\text { ppm }\end{array}$ & $\begin{array}{c}\mathrm{KNO}_{3} \\
\mathrm{ppm}\end{array}$ & $\underset{\%}{\mathrm{GdL}}$ & $\begin{array}{c}\text { Mikro- } \\
\text { kokken } \\
\text { Keime/g }\end{array}$ & $\begin{array}{c}\text { Lakto- } \\
\text { bazillen } \\
\text { Keime/g }\end{array}$ & $\begin{array}{c}\text { Askorb.- } \\
\text { Verb. } \\
\%\end{array}$ & Bemerkung \\
\hline
\end{tabular}

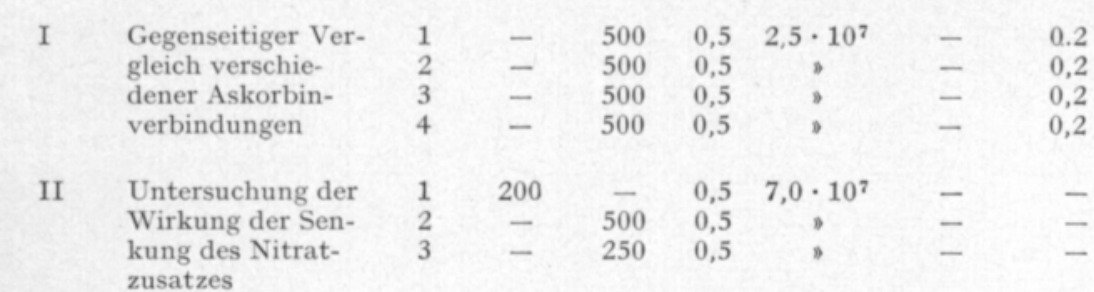

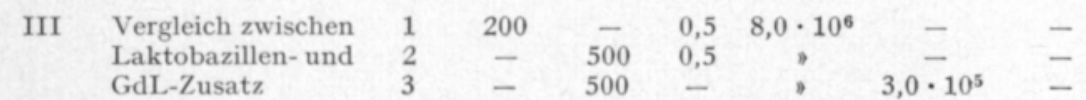

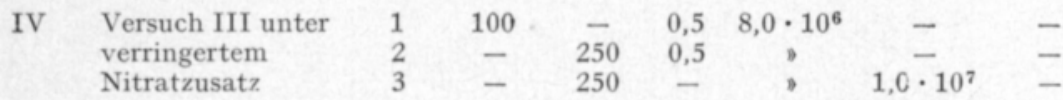

V Versuch IV bei noch niedrigerem

Nitratzusatz, Untersuchung der

Wirkung von

Erythorbat-Zusatz

VI Untersuchung der Wirkung von ausserordentlich geringem Nitrat- und Nitritzusatz bei gleichzeitigem Arbeiten mit Erythorbat

VII Untersuchung des Einflusses der Nitratkonzentration

$\begin{array}{lrl}- & \overline{62,5} & - \\ - & 125 & - \\ - & 250 & - \\ - & 500 & - \\ - & 1000 & -\end{array}$

$1,2 \cdot 10^{\circ}$

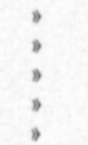

Einflusses der tration

IX

Untersuchung der Wirkung von Erythorbat bei extrem niedrigem und bei üblichem Nitritzusatz

X Untersuchung der Wirkung von Erythorbat bei extrem niedrigem und bei üblichem Nitratzusatz \begin{tabular}{rrr}
\hline & - & - \\
50 & - & - \\
100 & - & - \\
200 & - & - \\
400 & - & - \\
800 & - & -
\end{tabular}

$\begin{array}{ccc}2,0 \cdot 10^{6} & 1,0 \cdot 10^{7} & - \\ : & \vdots & - \\ : & \vdots & - \\ : & - & -\end{array}$

$\begin{array}{ccc}\overline{-} & - & - \\ \overline{25} & - & - \\ 25 & - & - \\ 200 & - & - \\ 200 & - & - \\ - & - & - \\ - & - & - \\ - & 50 & - \\ - & 50 & - \\ - & 250 & -\end{array}$
Askorbinsäure

Na-Erythorbat

Na-Askorbat

Askorbinsäure: Na-Erythorbat $1: 1$
Na-Erythorbat

$\mathrm{M}_{\mathrm{NaNO}_{2}} / \mathrm{M}_{\mathrm{KNO}_{3}}=34 / 50$

Na-Erythorbat

Na-Erythorbat $\begin{array}{cccc}1,0 \cdot 10^{7} & 5,0 \cdot 10^{6} & - & \\ & \vdots & 0,05 & \text { Na-Erythorbat } \\ & : & 0,05 & \text { Na-Erythorbat }\end{array}$

$\begin{array}{cccc}8,0 \cdot 10^{7} & 1,3 \cdot 10^{7} & - & \\ \vdots & \vdots & 0,05 & \text { Na-Erythorbat } \\ & \vdots & -\overline{05} & \text { Na-Erythorbat }\end{array}$ 
\begin{tabular}{lllllll}
\hline \multirow{2}{*}{ Versuch } & $\begin{array}{l}\text { Zweck des } \\
\text { Versuchs }\end{array}$ & $\begin{array}{l}\text { Wurst- } \\
\text { posten }\end{array} \mathrm{NaNO}_{2} \mathrm{KNO}_{3}$ & $\underset{\text { ppm }}{\mathrm{GdL}}$ & $\begin{array}{c}\text { Mikro- } \\
\text { kokken }\end{array}$ & $\begin{array}{c}\text { Lakto- } \\
\text { bazillen }\end{array}$ & $\begin{array}{c}\text { Askorb. } \\
\text { Verb. }\end{array}$
\end{tabular} Keime/g Keime/g \%

XI Untersuchung des Einflusses der Starterkultur-

Konzentration

Einflusses der

Starterkultur-

Behandlungsweise

Untersuchung des Temperatureinflusses

XV Einfluss der FleischLagerungsdauer

Untersuchung des Nitrit-NitratVerhältnisses

$\begin{array}{ccccccc}1 & - & - & - & - & - & - \\ 2 & 100 & - & - & - & - & - \\ 3 & - & 150 & - & - & - & - \\ 4 & 100 & - & - & 1,5 \cdot 10^{7} & 4,3 \cdot 10^{6} & - \\ 5 & - & 150 & - & 1,5 \cdot 10^{7} & 4,3 \cdot 10^{6} & - \\ 6 & 100 & - & - & 1,3 \cdot 10^{8} & 4,3 \cdot 10^{7} & - \\ 7 & - & 150 & - & 1,3 \cdot 10^{8} & 4,3 \cdot 10^{7} & -\end{array}$

- 250

- 250

- 250

- 250

- 250

250

$$
\begin{aligned}
& - \\
& - \\
& - \\
& -
\end{aligned}
$$

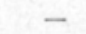

- $3,6 \cdot 10^{6}$

$2,4 \cdot 10^{6}$

$7,2 \cdot 10^{6}$

- $3,6 \cdot 10^{6}$

$2,4 \cdot 10^{6}$

$2,2 \cdot 10^{6}$
$-\quad 2$

-
-
$\overline{-}$
$1,2 \cdot 10^{7}$
$1,2 \cdot 10^{7}$
$2,8 \cdot 10^{7}$

-
-
-
$1,2 \cdot 10^{7}$
$1,2 \cdot 10^{7}$
$2,8 \cdot 10^{7}$

250

$\overline{171}$

-
-
-
-
-
-
-

$3,7 \cdot 10^{7}$

$1,0 \cdot 10^{7}$

,

$\overline{171}$

$\overline{171}$

171

171

250

- 250

$$
-
$$

$$
250
$$$$
-
$$$$
250
$$

$\begin{array}{rc}- & - \\ 0 & 250 \\ 25 & 213 \\ 50 & 177 \\ 100 & 104 \\ 171 & -\end{array}$

$\bar{z}$
$\bar{z}$

$2,5 \cdot 10^{7}$

$6,0 \cdot 10^{6}$

:

$$
\begin{aligned}
& - \\
& - \\
& - \\
& - \\
& -
\end{aligned}
$$$$
\begin{gathered}
- \\
- \\
- \\
- \\
- \\
-
\end{gathered} \quad\left\{\begin{array}{c}
2 \mathrm{Tage} / 26^{\circ} \rightarrow 2 / 24^{\circ} \rightarrow 2 / 22^{\circ} \\
1 \text { Tag } / 20^{\circ} \rightarrow / 15^{\circ} \\
2 \text { Tage } / 24^{\circ} \rightarrow 2 / 22^{\circ} \rightarrow 2 / 20^{\circ} \\
1 \text { Tag } / 18^{\circ} \rightarrow / 15^{\circ} \\
2 \text { Tage } / 22^{\circ} \rightarrow 2 / 20^{\circ} \rightarrow 3 / 18^{\circ} \\
\rightarrow / 15^{\circ} \\
2 \text { Tage } / 20^{\circ} \rightarrow 5 / 18^{\circ} \\
\rightarrow \\
7 \text { Tage } / 15^{\circ} \rightarrow / 18^{\circ} \rightarrow / 15^{\circ} \\
7
\end{array}\right.
$$

XVI Einfluss des Glukosegehalts

$\begin{array}{lcccccc}- & 150 & - & 1,2 \cdot 10^{7} & 1,4 \cdot 10^{7} & - \\ - & 150 & - & & & - \\ - & 150 & - & & & - \\ - & 150 & - & & & - \\ - & 150 & - & : & & -\end{array}$

$\begin{array}{lll}- & 0,0 \% & \text { Glukose } \\ - & 0,2 \% & \\ - & 0,4 \% & \\ - & 0,6 \% & \\ - & 0,8 \% & \end{array}$

Tabelle 5. Die variierenden Zusatzmittelmengen und Starterkulturen in den vergleichenden Versuchen (Versuchsreihen V1 - V6) und in den Salmonellenversuchen (Versuchsreihen S1-S2).

\begin{tabular}{cccccccc}
\hline $\begin{array}{c}\text { Wurst- } \\
\text { posten }\end{array}$ & $\begin{array}{c}\mathrm{NaNO}_{2} \\
\text { ppm }\end{array}$ & $\begin{array}{c}\mathrm{KNO}_{3} \\
\text { ppm }\end{array}$ & $\begin{array}{c}\mathrm{GdL} \\
\%\end{array}$ & $\begin{array}{c}\text { Glukose } \\
\%\end{array}$ & $\begin{array}{c}\text { Na-Erythorb. Mikrokokken } \\
\%\end{array}$ & $\begin{array}{c}\text { Laktobazillen } \\
\text { Keime/g }\end{array}$ & \begin{tabular}{c} 
Keime/g \\
\hline
\end{tabular} \\
\hline & 100 & - & 0,6 & 0,3 & 0,05 & - & - \\
2 & 200 & - & 0,6 & 0,3 & 0,05 & - & - \\
3 & - & 150 & - & 0,5 & 0,05 & $1,0 \cdot 10^{7}$ & $1,0 \cdot 10^{7}$ \\
4 & - & 300 & - & 0,5 & 0,05 & $1,0 \cdot 10^{7}$ & $1,0 \cdot 10^{7}$ \\
5 & 50 & 75 & & 0,5 & 0,05 & $1,0 \cdot 10^{7}$ & $1,0 \cdot 10^{7}$ \\
\hline
\end{tabular}




\section{3 Material und Verfahren}

\subsubsection{Rohstoffe und Herstellung der Versuchswürste}

Im folgenden werden die Grundrezeptur und das Herstellungsverfahren der Würste beschrieben, die dann im Rahmen der Versuche auf die in den Tabellen 4 und 5 dargelegte Weise modifiziert wurden. Die Grundrezeptur der Versuchswürste geht aus Tabelle 6 hervcr.

Tabelle 6. Grundrezeptur der Versuchswürste.

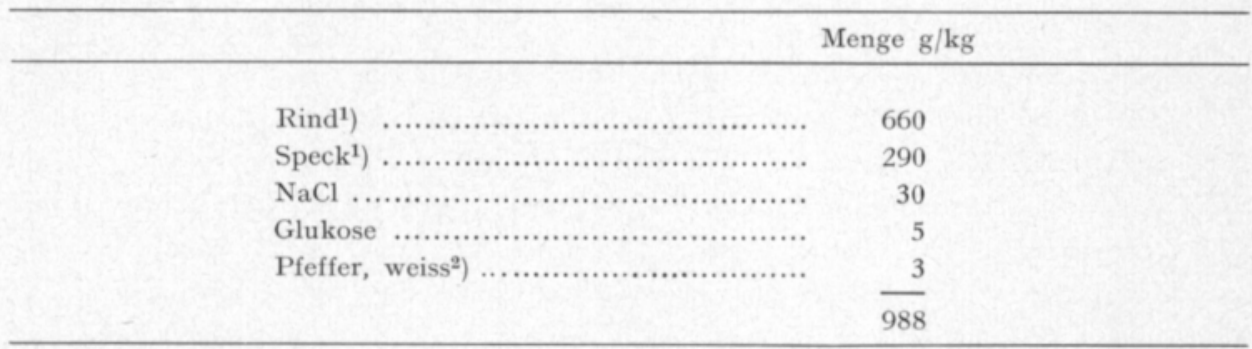

1) siehe Text

$\left.{ }^{2}\right)$ Firma Wiberg, Salzburg, Österreich

Als Rindfleisch wurde entsehntes Kuhfleisch mit einem Fettgehalt $<15 \%$ verarbeitet; als Speck diente fester Rückenspeck vom Schwein. Die Fleischsorten waren in der Versuchsfabrik des Instituts für Fleischtechnologie der Universität Helsinki zerlegt und eingefroren worden.

Je nach Versuchsanordnung wurde die in Tabelle 6 zusammengestellte Grundmenge mit Starterkulturen, GdL oder Wasser auf $1000 \mathrm{~g}$ aufgefüllt. Nitrit, Nitrat und Askorbinverbindungen wurden bei der Berechnung der Brätzusammensetzung mengenmässig nicht berücksichtigt. In den Versuchsreihen I-VI betrug der Salzgehalt $35 \mathrm{~g} / \mathrm{kg}$; da dies — verglichen am allgemeinüblichen Wert - eine grosse Menge ist, wurden in den übrigen Versuchen nur $30 \mathrm{~g} / \mathrm{kg}$ zugesetzt. In Verbindung mit Nitrit wurden die Askorbinverbindungen separat eingewogen, so dass sie erst beim Kuttern mit ersterem zu reagiercn vermochten.

Zur Herstellung der Wurstmasse wurde das Rindfleisch zwanzig Stunden vor dem Kuttern aus einem Raum von $-18^{\circ} \mathrm{C}$ in einen Raum von $+2^{\circ} \mathrm{C}$ gebracht. Am Herstellungstag wurden das Fleisch und der Speck mit einer Bandsäge zerkleinert. Vor dem Kuttern des ersten Postens wurde der Kutter (Seydelmann Rasant K-40, Hersteller Fa. Seydelmann, Stuttgart, Bundesrepublik Deutschland) mit einem Salz-Eis-Gemisch gekühlt.

Das Fleisch wurde zunächst drei Umdrehungen bei niedriger Drehzahl gekuttert; dabei wurden die Zusatzmittel und Starterkulturen zugesetzt. Während der folgenden zwei Umdrehungen erfolgte die Speckzugabe, und zum Abschluss wurde das Brät noch fünf Umdrehungen im Schnellgang gekuttert. Bei Beendigung des Kutterns hatte das Brät eine Temperatur zwischen $-1^{\circ}$ und $+1^{\circ} \mathrm{C}$. Das Brät wurde zu etwa $600 \mathrm{~g}$ schweren Würsten, 
pro Posten 6-8 Würste, in Naturin-Darm, Ø $68 \mathrm{~mm}$, (Hersteller AB Schaub \& Co., Stockholm, Schweden) gefüllt. Nach dem Waschen wurden die Würste in die Reifekammer (Hessteller Autotherm, Waxweiler, Bundesrepublik Deutschland) gebracht, wo sie zwecks Stabilisierens und Abtrocknens der Oberfläche 6-8 Stdn. ohne Heizung und Befeuchtung belassen wurden (HEChelmann 1973, mündliche Information, RöDEl 1973). Danach wurde die Reifekammer auf eine Temperatur von $20^{\circ} \mathrm{C}$ und eine Feuchte von $96 \% \mathrm{rF}$ eingestellt. Nach zwei Tagen wurde die Feuchte auf $94 \% \mathrm{rF}$ gesenkt und mit dem Räuchern begonnen. Im Alter von sieben Tagen kamen die Würste ins Nachreifelager, das eine Temperatur von $15^{\circ} \mathrm{C}$ und eine Feuchte von $83 \% \mathrm{rF}$ aufwies.

In den vergleichenden Versuchen und Salmonellenversuchen betrug die Temperatur in den ersten beiden Tagen $22^{\circ} \mathrm{C}$; danach estsprach das Temperaturprogramm dem obigen. Die höheren Temperaturen sind besser im Zusammenhang mit GdL geeignet, jedoch werden neuerdings bei Einsatz von Starterkulturen auch höhere Temperaturen gefahren (CORETTI 1975).

Dieser Umstand liess sich bei der Auswertung der Ergebnisse lediglich in der Form berücksichtigen, dass V $₫$ rgleiche der Einflüsse zu untersuchender Faktoren im wesentlichen nur innerhalb der einzelnen Versuchsreihe angestellt wurden.

In den Versuchsreihen I-XII kamen selbstgezüchtete Starterkulturen zum Einsatz; die Stämme waren die gleichen wie in den handelsüblichen Starterkıltur-Präparaten (Duploferment 66, Hersteller Rudolf Müller \& Co., Giessen, Bundesrepublik Deutschland). Die Starterkultur bestand aus Micrococcus $s p$. (NirnivaAra 1955) und Lactobacillus plantarum im Verhältnis $1: 1$. Die zugesetzte Bakterienzahl betrug $10^{7} \mathrm{Keime/g}$ (Nurmi $1966 \mathrm{a}$ ). Die Züchtung der Mikrokokken begann durch Beimpfung von $50 \mathrm{ml}$ Nährlösung (Merck, Darmstadt, Bundesrepublik Deutschland, Nr. 7882) mit einer Kolonie des betreffenden Organismus und 20stündiges Bebrüten der Lösung bei $30^{\circ} \mathrm{C}$. Danach wurde diese Lösung in eine grössere Menge (1-2 l) der gleichen Nährlösung gegeben und 48 Stunden inkubiert. Nach dem Zentrifugieren und Waschen wurde die Suspension eingefroren.

Die Züchtung der Laktobazillen erfolgte in analoger Weise unteı Verwendung von SL-Brühe (Merck Nr. 5413 ohne Agar-Agar). In den übrigen Versuchsreihen fanden vom Duploferment-Hersteller gelieferte gefriergetrocknete Mikrokokken und Laktobazillen Verwendung.

Beim GdL und den Askorbinverbindungen handelte es sich um technische Qualitäten (Hersteller: Fujisawa Pharmaceutical Co., Ltd. Japan).

\subsection{Chemische Verfahren}

Vorbehandlung der Probe: Etwa $300 \mathrm{~g}$ Brät oder Wurst wurden zweimal durch den Wolf (Hersteller: Koneteollisuus Oy, Helsinki Finnland) gedreht. Bei der Stickoxidmyoglobin-Bestimmung erfolgte das Zerkleinern in einem verdunkelten Raum.

Wassergehalt: Etwa $3 \mathrm{~g}$ Probe wurden in einen tarierten Aluminiumtiegel eingewogen und anderthalb Stunden auf $135^{\circ} \mathrm{C}$ gehalten. Aus der Gewichtsabnahme wurde der Wassergehalt berechnet. 
Fettgehalt:Die Bestimmung des Fettgehaltes erfolgre nach dem Verfahren von Gerber unter Verwendung eines 5-g-Butyrometers.

Salzgehalt: Die Bestimmung des Salzgehalts erfolgte durch $\mathrm{AgNO}_{3}$-Titration unter Verwendung von $\mathrm{K}_{2} \mathrm{Cr}_{2} \mathrm{O}_{7}$-Lösung als Ind'kator.

Nitrit- und Nitratgehalt: Die Bestimmung des Nitıit- und Nitratgehalts erfolgte nach dem von STOyA (1969) modifizierten Verfahren von GRAU und Mirna (1957). In den vergleichenden Versuchen wurden der Probe jeweils 2,5 ml 5prozentige $\mathrm{HgCl}_{2}$-Lösung zugesetzt um eine durch die Askorbinsäure bewirkte Abnahme der Nitritmenge während der Analyse zu verhindern (MIRNA und SchÜTZ 1970).

Von jeder Wurst wurden zwei Proben entnommen; in der FarbreaktionsPhase wurden für jede Lösung zwei Parallelbestimmungen durchgeführt. Jede Einzelbestimmung erfolgte unter Einbeziehung des Nitritstandards und des Nitratstandards, an Hand deren zum einen der während der Reduktion eingetretene Nitritschwund, zum anderen die Intensität der Nitratreduktion berechnet wurden. Auf der Basis der von den Standardlösungen gelieferten Korrekturfaktoren wurde dann der Nitrat- und Nitrif gehalt der Probe berechnet. Als Spektralphotometer diente ein Hitachi 191 Digital (Hersteller: Hitachi Ltd., Tokio, Japan). In den Versuchsreihen I-VI wurde lediglich der Nitritgehalt, in den übrigen Versuchsreihen der Nitrit- und Nitratgehalt bestimmt.

Stickoxidmyoglobin und -myochromogen: Die Stickoxidmyoglobin- und -myochromogen-Konzentration wurde als Summenwert nach der von Mirna und Schutz (1972) aus dem Verfahren von Hornsey (1956) entwickelten Methode bestimmt. Zur Homogenisierung der Proben diente ein UltraTurrax-Gerät (Hersteller: Janke et Kunkel, Staufen i. Br., Bundesrepublik Deutschland). Nach der Azetonextraktion wurde die Probe erst durch acht Filterpapiere (Whatman Nr. 4) und dann noch einmal durch sechs Filterpapiere (Schleicher et Schüll, Nr. 1575) hindurch filtriert. Die Extinktion der Probelösung wurde mit einem Spektralphotometer Hitachi 191 gemessen; das Ergebnis gibt die Summenkonzentration von Stickoxidmyoglobin und Stickoxidmyochromogen an. Eine solche Bestimmung erfolgte in den Präliminarversuchsreihen IV-VII und in den vergleichenden Versuchsreihen V1-V6.

\section{3.3 Mikrobiologische Verfahren}

Die verwendeten Nährböden sowie die Inkubationstemperaturen und -zeiten gehen aus Tabelle 7 hervor.

Als Probe wurden $10 \mathrm{~g}$ Brät bzw. Wurst in $90 \mathrm{ml}$ physiologische Kochsalzlösung gegeben und im Ultra-Turrax-Homogenisator eine Minute lang homogenisiert. Von jeder Probe wurden zwei Parallelbestimmungen durchgeführt. Beim Salmonellenversuch hatte die Probe ein Volumen von $5 \mathrm{~g} / 45 \mathrm{ml}$; an ihr wurde nur einè Bestimmung durchgeführt. Im SL-Nährboden (Rogosa-Agar) gedeihen auch gewisse Organismen der Gattungen Pediococcus, Leuconostoc und Streptococcus (Kitchell und Shaw 1975). Der Chapman-Nährboden dient eigentlich zur Feststellung pathogener Staphylokokken, jedoch wachsen auf ihm auch nichtpathogene Staphylokokken und Mikrokokken (Niskanei und Nurmi 1976). Die auf Plattenkulturen gediehenen Bakterien wurden jedoch nicht näher identifiziert. 
Tabelle 7. Nährböden sowie Inkubationstemperaturen und -zeiten.

\begin{tabular}{llll}
\hline Mikrobengruppe & Nährboden & \multicolumn{1}{c}{ Hersteller } & $\begin{array}{c}\text { Inkubations- } \\
\text { temperatur und -zeit }\end{array}$ \\
\hline Gesamtbakterienzahl & Plate-count-Agar & $\begin{array}{l}\text { Merck no. 5463 } \\
\text { (Merck, Darmstadt) }\end{array}$ & $30^{\circ} \mathrm{C} / 4$ Tage \\
Laktobazillen & Rogosa (SL) & Merck no. 5413 & $30^{\circ} \mathrm{C} / 4$ Tage \\
Mikrokokken & Chapman Agar & Difco no. 0297 & $30^{\circ} \mathrm{C} / 2$ Tage \\
& Staphylococcus & & \\
Fäkale Strepto- & medium no. 110 & Orion Oy, & $37^{\circ} \mathrm{C} / 1$ Tag \\
kokken & Slanetz- und & Helsinki & \\
Kolibakterien ${ }^{1}$ ) & Bartley-Agar & Difco no. 0012-01 & $37^{\circ} \mathrm{C} / 1$ Tag \\
Hefen und & VRB & Orion Oy, & $25^{\circ} \mathrm{C} / 4$ Tage \\
Schimmelpilze & Sabouraud- & Helsinki & $35^{\circ} \mathrm{C} / 1$ Tag \\
Salmonellen $\left.{ }^{2}\right)$ & Maltose-Agar & Orion Oy, & \\
\hline
\end{tabular}

\footnotetext{
1) nur in den vergleichenden Versuchen

2) nur in den Salmonellenversuchen
}

\subsubsection{Physikalische Verfahren}

$p H$-Wert: Der $\mathrm{pH}-$ Wert wurde unmittelbar an der Wurst mit einem $\mathrm{pH}$ Messgerät Findip 555A (Hersteller: Prolab Oy, Helsinki, Finnland) mit Kombinationselektrode gemessen.

$a_{\mathrm{w}}$-Wert: Zur Bestimmung des $\mathrm{a}_{\mathrm{w}}$-Wertes diente ein $\mathrm{a}_{\mathrm{w}}$-Messgerät Sina AMT (Hersteller: Sina AG, Zürich, Schweiz). An jeder Probe wurden zwei Parallelbestimmungen durchgeführt. Die vom Gerät angezeigten Ergebnisse wurden für beide Fühler getrennt an Hand der von gesättigter $\mathrm{KNO}_{3}$-Lösung gelieferten Ablesung (theoretischer Wert 0.920) korrigiert.

Konsistenz: Die Konsistenz wurde mit einem Gerät Instron Universal Testing Machine TM-M (Hersteller: Instron Ltd., High Wycombe, England) gemessen. Der Wurst wu1 de hierzu ein $6 \mathrm{~cm}$ langes Stück entnommen und mit einem Kolben, Ø $60 \mathrm{~mm}$, senkrecht zu seiner Längsachse um $1 \mathrm{~cm}$ zusammengepresst. Dieser Pressvorgang wurde dreimal durchgeführt; der Konsistenzwert $(\mathrm{kg})$ wurde als Mittelwert aus diesen Einzelmessungen berechnet.

Gezvichtsverlust: Von jedem Wurstposten wurden zwei Würste auf $1 \mathrm{~g}$ genau gewogen und an den Stichtagen auf ihr Gewicht kontrolliert.

Photos: An den Analysentagen wurden von den Würsten mit einer Canon Canoflex (Hersteller: Canon Camera Co., Japan) unter Verwendung von Kinefilm Kodak Ektachrome-x 19 DIN und zwei Elektronenblitzgeräten Farbaufnahmen gemacht.

\subsubsection{Organoleptische Methoden}

Oberflächenfarbe, Schnittflächenfarbe, Konsistenz, Geruch und Geschmack der Würste der vergleichenden Versuchsreihen wurden nach dem in Tabelle 8 dargelegten Punktbewertungssystem beurteilt. Für den Zeitpunkt 14 Tage nach Versuchsbeginn wurden ferner gewogene Gesamtpunktwerte berechnet, 
wobei der maximale Punktwert der Farbe 6, der des Geschmacks 4, der der Konsistenz und des Geruchs jedoch nur 2 betrug.

Die Farbbeständigkeit wurde an Hand einer Rangordnungsprüfung durch Vergleich von ein, zwei und drei Stunden alten, unter Lichteinwirkung gelagerten Schnittflächen mit frischer Schnittfläche geschätzt.

An der Beurteilung nahmen jeweils 6-9 Personen teil.

Tabelle 8. Punktsystem der organoleptischen Beurteilung.

\begin{tabular}{|c|c|c|c|}
\hline $\begin{array}{l}\text { Oberflächen- und } \\
\text { Schnittflächenfarbe }{ }^{1} \text { ) }\end{array}$ & Konsistenz & Geruch & Geschmack \\
\hline $\begin{array}{l}\text { 6: dunkelrot, } \\
\text { hell }\end{array}$ & 2: fest & $\begin{array}{l}\text { 2: } \text { frisch, } \\
\text { aromatisch }\end{array}$ & $\begin{array}{l}\text { 4: aromatisch, } \\
\text { frisch }\end{array}$ \\
\hline $\begin{array}{l}\text { 0: grau, } \\
\text { farblos }\end{array}$ & $\begin{array}{l}0: \text { roh, } \\
\text { weich }\end{array}$ & $\begin{array}{l}\text { 0: Geruchs- } \\
\text { fehler, } \\
\text { roh }\end{array}$ & $\begin{array}{l}\text { 0: Geschmacks- } \\
\text { fehler, } \\
\text { roh }\end{array}$ \\
\hline
\end{tabular}

1) Fehler: ungleichmässige, graue oder braune Farbe

\section{3. 6 Verarbeitung der Ergebnisse}

Auf der Basis der Mittelwerte der in den vergleichenden Versuchsreihen für die einzelnen Wurstposten erhaltenen Ergebnisse wurde für die Zeitpunkte drei und 14 Tage je eine Varianzanalyse durchgeführt. Die Gesamtmittelwerte von sechs Versuchen wurden nach der Methode von Tukey miteinander verglichen. In den Tabellen sind die Signifikanz des Unterschiєds (S.d.U.) sowie der kleinste signifikante Unterschied (k.s.U.) angegeben. Die Resultate der Rangordnungsprüfung wurden an Hand der Tabellen von Kramer (KAHAN u.a. 1973) getestet.

Insbesondere was die organoleptischen Beurteilungen betrifft, ist also zu bemerken, dass die Varianzanalyse auf der Grundlage von Mittelwerten duıchgeführt wurde, wobei der einzelne Tabellenwert 6-9 Beurteilungs-Ergebnisse und der Gesamtmittelwert somit 36-54 Einzelergebnisse repräsentierte, was eine Sicherung der Signifikanz der Ergebnisse bedeutet.

\section{4 Ergebnisse der Präliminarversuche}

Durch die vorbereitenden Versuchsreihen sollten zum einen die Nitritund Nitratkonzentrationen für die dann folgenden vergleichenden und Salmonellenversuchsreihen ermittelt werden, zum anderen sollte aber auch der Einfluss verschiedener technologischer Verfahrensweisen nicht nur auf die Restnitrit- und -nitratmengen, sondern auch auf die Eigenschaften der Rohwurst im allgemeinen klargestellt werden. Da alle Versuche doppelt ausgeführt und im Rahmen jedes Einzelversuchs mehrere der im Abschnitt 3. 3. aufgeführten Bestimmungen durchgeführt wurden, fiel Ergebnismaterial im Umfang von etwa 500 Tabellen an. Da es wenig sinnvoll erschien, diese umfangreichen 
Unterlagen hier in ihrer Gesamtheit wiederzugeben, enthält die vorliegende Arbeit nur die wichtigsten Ergebnisse, dargeboten entweder in graphischer, tabellarischer oder verbaler Form.

\section{4.1 Nitrit- und Nitratzusatz}

Der Einfluss des Nitritzusatzes wurde in der Vers u chs reihe VI I I, der Einfluss des Nitratzusatzes in der Versuchsreihe VII untersucht. Auch bei den Versuchsreihen II-VI, IX, X und XIV lagen innerhalb der gleichen Versuchsreihe verschiedene Nitrit- und Nitratzusätze vor. In den übrigen Versuchen lagen die Nitrit- und Nitratzusätze zwar unter dem üblichen Wert, eine eigentliche Untersuchung ihrer Auswirkungen unterblieb hier jedoch.

Nach den Ergebnissen der Versuchsreihe VIII hatte der Nitritzusatz sehr starken Einfluss auf die Bakterienflora der Würste. Da letzteren Starterkulturen zugesetzt worden waren, folgte die Gesamtbakterienzahl (Bild 6) weitgehend den quantitativen Schwankungen der Laktobazillen und Mikrokokken.

Hinsichtlich der Laktobazillen gliederten sich die Würste in drei Gruppen (Bild 7). Bei den Würsten 1,2 und 3 stieg die Laktobazillenzahl rasch und auf

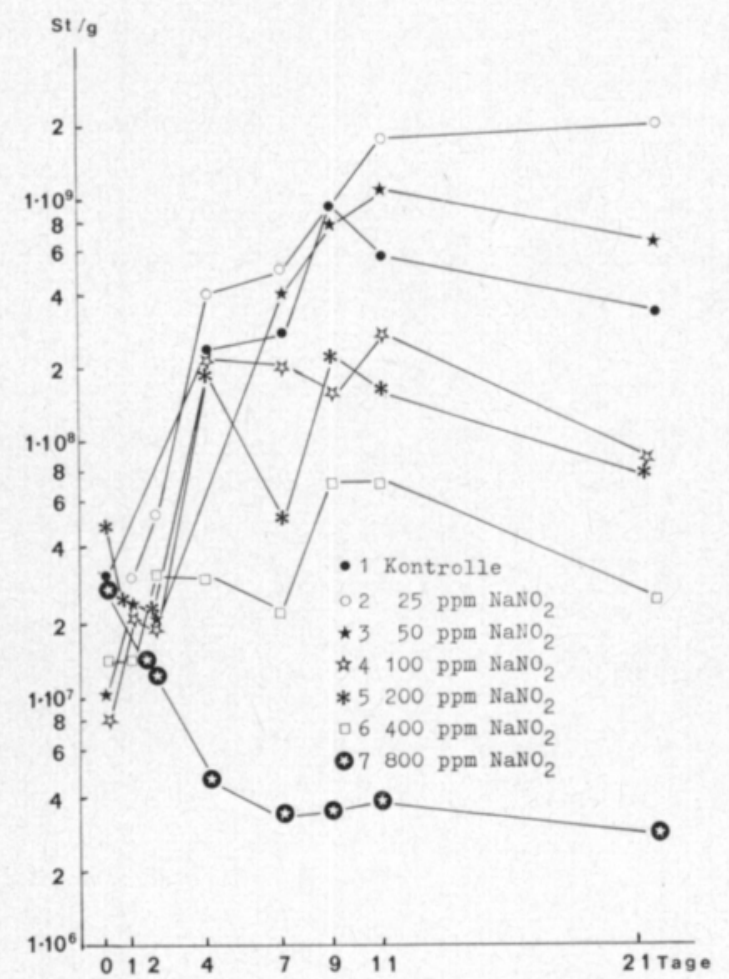

Bild 6. Einfluss der zugesetzten Nitritmenge auf die Gesamtbakterienzahl. Versuchsreihe VIII B.

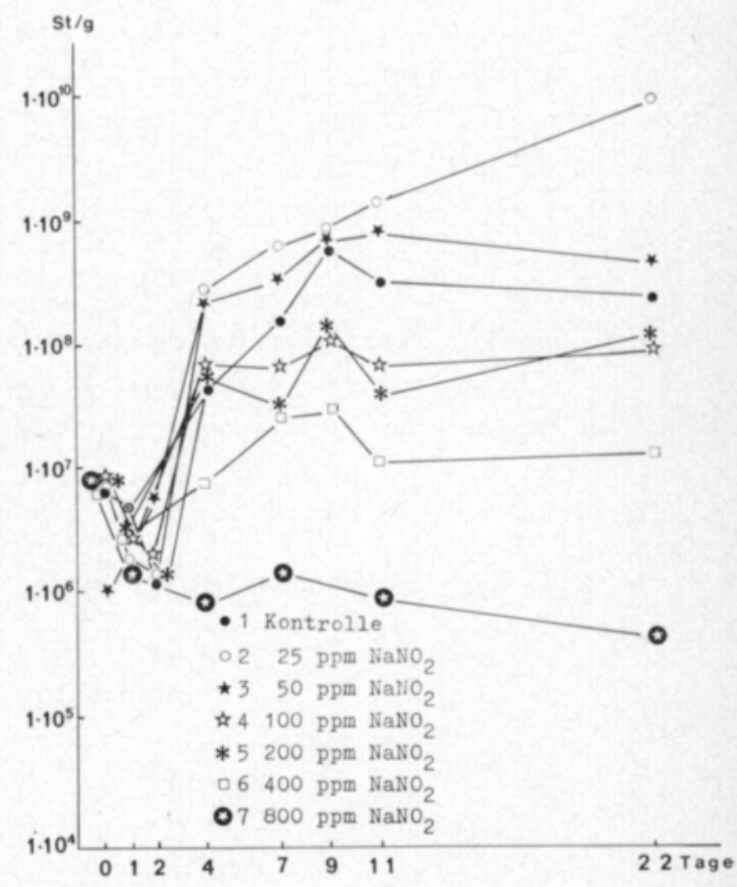

Bild 7. Einfluss der zugesetzten Nitritmenge auf die Laktobazillenzahl. Versuchsreihe VIII B. 
einen hohen Wert an, wenngleich bei der Wurst 3 gegenüber der Wurst 2 eine geringfügige Hemmwirkung zu beobachten war.

Bei den Würsten 4-6 stieg die Laktobazillenzahl anfangs an, blieb aber unter den Werten der vorangehenden Gruppe. Danach kam das Wachstum zum Erliegen. Der höchste Nitritzusatz (Wurst 7) bewirkte bereits eine recht starke Abnahme der Laktobazillenzahl.

Bei den Mikrokokken (Bild 8) kam es zu keiner eigentlichen Vermehrungsperiode; ihre zahlenmässige Abnahme war je nach Nitritzusatz verschieden. Das konsequenteste sichtbare Ergebnis nach Bild $\mathbf{8}$ war, dass bei dem höchsten Nitritzusatz am meisten Mikrokokken vernichtet wurden. Die Resultate der übrigen Würste waren, was den Nitritzusatz betrifft, derart inkonsequent, dass es sich bei den zahlenmässigen Änderungen der Mikrokokken offensichtlich um indirekte, vorwiegend über die Laktobazillen wirksam gewordene $\mathrm{pH}$ Wert-Einflüsse handelte. Bei den Würsten $\mathbf{3 - 5}$ sank die Mikrokokkenzahl in der gleichen Reihenfolge wie der $\mathrm{pH}$-Wert (Bild 10), während bei den Würsten 1, 2 und 6 die Mikrokokkenzahl eine eher indifferente Resultierende aus den Wirkungen von $\mathrm{pH}-$ Wert und Nitritzusatz darstellte.

Bild 8. Einfluss der zugesetzten Nitritmenge auf die Mikrokokkenzahl. Versuchsreihe VIII B.

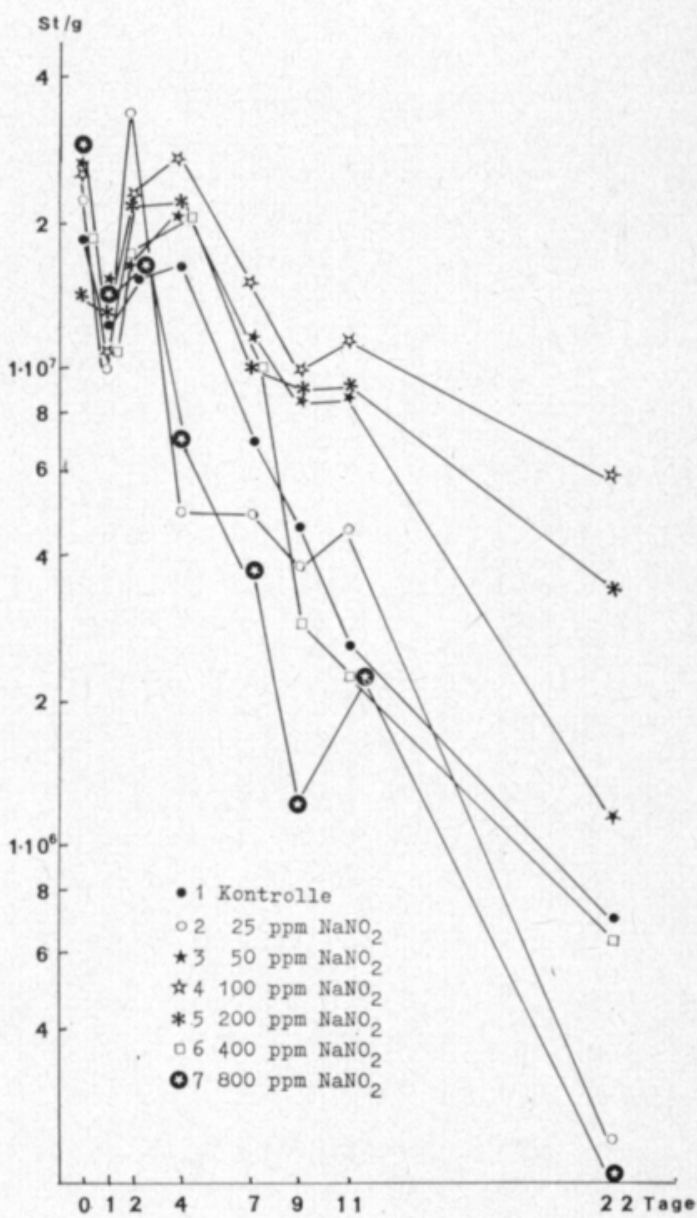


Die fäkalen Streptokokken erreichten in den Würsten 1 und 2 zahlenmässig einen ziemlich hohen Wert $\left(10^{3}-10^{4}\right.$ Keime/g). Im Versuch B hatte auch die Wurst 3 die ganze Zeit über einen hohen Gehalt an fäkalen Streptokokken (etwa $10^{3} \mathrm{Keime} / \mathrm{g}$ ). Bei den übrigen Würsten bewegte sich der entsprechende Wert um etwa $100 \mathrm{Keime/g.} \mathrm{Den} \mathrm{Auszählungsergebnissen} \mathrm{zufolge} \mathrm{wurde} \mathrm{das}$ Wachstum der Hefen durch Nitrit - freilich nur leicht - gehemmt. Allerdings war in beiden Versuchsreihen der Hefegehalt zu Beginn so gering, dass eine eventuelle Wirkung des Nitrits nicht zum Tragen kommen konnte.

Der Nitritgehalt (Bild 9) ging bei allen Nitritzusätzen rasch zurück. Bei den Würsten 6 und 7 blieb ein ziemlich hoher Restnitritgehalt bestehen. Der Korrelationskoeffizient zwischen zugesetztem und Restnitrit betrug $\mathrm{r}=+0,992$.

Der Nitratgehalt erreichte vor allem bei der Wurst 7, aber auch bei der Wurst 6 (Tabelle 9) einen recht hohen Wert. Das entstandene Nitrat wurde dann kaum noch zu Nitrit reduziert.

Der deutlichste Einfluss des Nitritzusatzes zeigte sich bei der Entwicklung des pH-Werts. Der End-pH folgte grob gesehen der Reihenfolge der Nitrit-

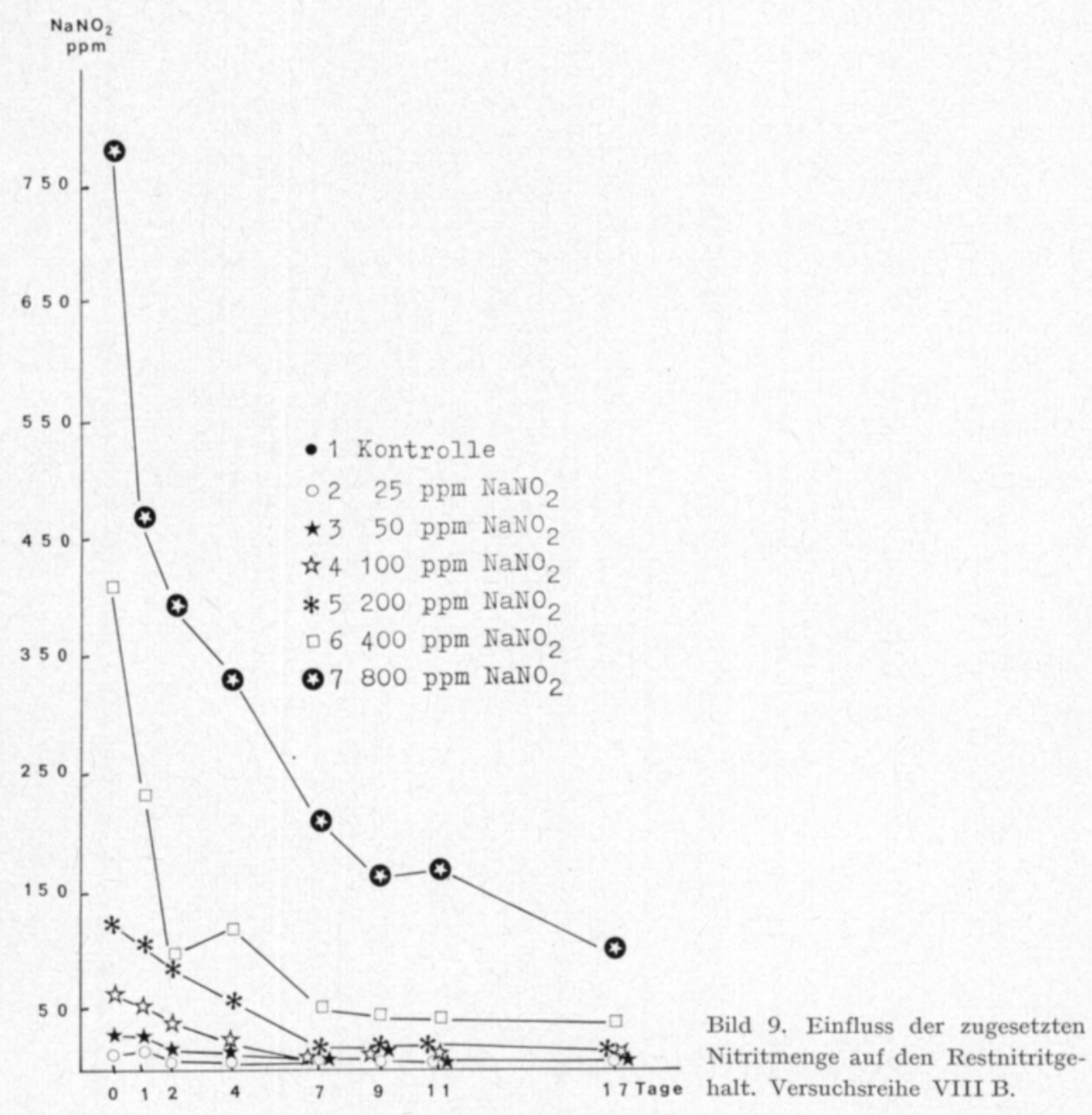


Tabelle 9. Nitratgehalte der Würste der Versuchsreihe VIII B (ppm).

\begin{tabular}{|c|c|c|c|c|c|c|}
\hline \multirow[b]{2}{*}{ Zeit (Tage) } & \multirow[b]{2}{*}{2} & \multicolumn{4}{|c|}{ Wurstposten ${ }^{2}$ ) } & \multirow[b]{2}{*}{7} \\
\hline & & 3 & 4 & 5 & 6 & \\
\hline 0 & 0,0 & 0,0 & 10,4 & - & 79,2 & 116,3 \\
\hline 1 & 0,0 & 13,4 & 41,3 & 87,8 & 197,3 & 268,9 \\
\hline 9 & 17,7 & 28,9 & - & 39,9 & 94,3 & 224,5 \\
\hline 22 & 13,7 & 13,1 & 14,5 & 24,0 & 92,9 & 230,6 \\
\hline
\end{tabular}

1) Siehe Tabelle 4

zusätze (Bild 10). Bei den Würsten 1 und 2 lag der End-pH sehr niedrig, bei den Würsten 3-6 etwa im normalen Bereich und bei der Wurst $7 \mathrm{zu}$ hoch. Die Elektrode des pH-Messgeräts war beim Versuch B an den ersten beiden Stichtagen defekt; aus diesem Grunde fehlen in Bild 10 diese Ergebnisse. Versuch A lieferte gleichartige Ergebnisse wie Versuch B, jedoch waren die Unterschiede bei ersterem geringer. Am Ende des Versuchs wurden die niedrigsten $\mathrm{pH}$-Werte bei den Würsten $1(4,70)$ und $2(4,75)$, die höchsten $\mathrm{pH}$ Werte bei den Würsten $6(4,92)$ und $7(5,00)$ festgestellt.

Die Würste $\mathbf{2 - 5}$ hatten bereits am zweiten Tag eine leuchtendrote Farbe. Bei den Würsten 6 und 7 war die Farbe trüber, ausserdem wiesen sie

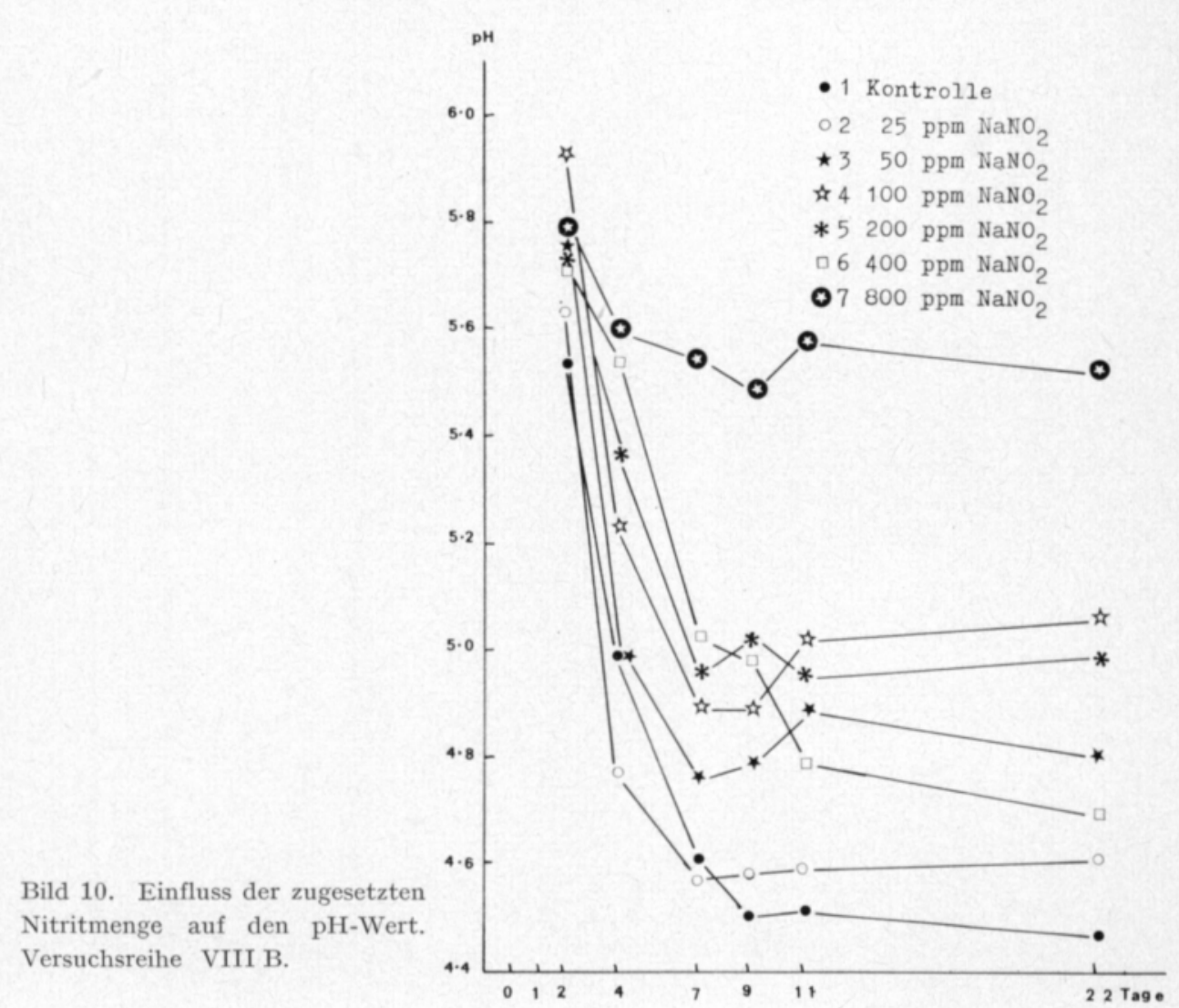


eine dicke graue Oberflächenschicht auf. Zum Versuchsende hin erfolgte auch in dieser Oberflächenschicht eine Umrötung, die Kernfarbe blieb jedoch matt. Bei Zusatz von Nitrit war diese graue Oberflächenschicht in sämtlichen Versuchsreihen dieser Untersuchung zu beobachten. Die übrigen Würste hatten eine einwandfreie Farbe.

Der Einfluss des Nitratzusatzes wurde in der Versuchsreihe VII untersucht. Hinsichtlich der Gesamtbakterienzahl unterschieden sich die Würste 1 und 2 deutlich von den übrigen Würsten. Neun Tage nach Versuchsbeginn setzte ein steiler Anstieg der Bakterienzahl ein (Bild 11). Bei den Würsten 5 und 6 ist eine die Gesamtbakterienzahl senkende Wirkung des Nitrats zu erkennen. In SL-Nährboden (Bild 12) wurden deutlichere systematische Unterschiede als in PC-Nährboden (Bild 11) beobachtet. Zwischen dem ersten und zweiten Tag fand in den Würsten 1 und 2 eine rege Bakterienvermehrung statt. Ein Vergleich der Bilder 11 und 12 zeigt, dass nicht alle Laktobazillen in PC-Agar gediehen, denn in SLNährboden entwickelten sich, vor allem was die Würste 1 und 2 betrifft, mehr Bakterien als in PC-Agar.

Die Vermehrung der Laktobazillen in den Würsten 1 und 2 lief parallel zu schnellem pH-Wert-Abfall (Bild 16). Bei Versuchsende hatten die Würste

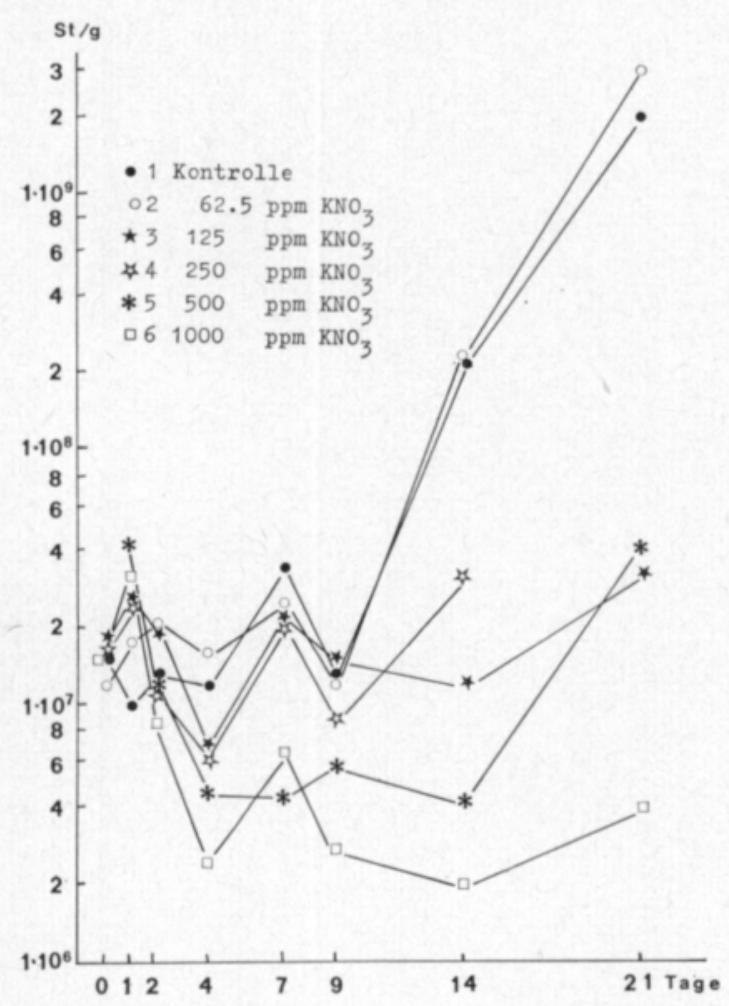

Bild 11. Einfluss der zugesetzten Nitratmenge auf die Gesamtbakterienzahl. Versuchsreihe VII B.

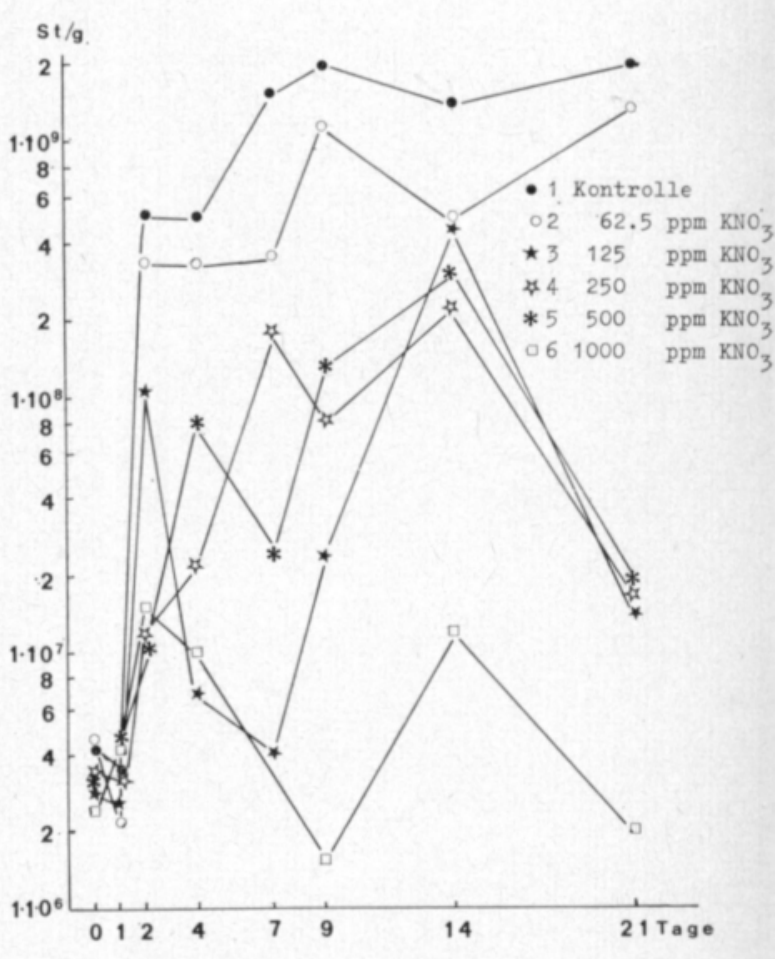

Bild 12. Einfluss der zugesetzten Nitratmenge auf die Laktobazillenzahl. Versuchsreihe VII B. 
1 und 2 einen etwa hundertmal höheren Laktobazillengehalt als die übrigen Würste; bei den Würsten 3-5 waren die Differenzen gering und schienen auf Zufälligkeit zu beruhen. Die Wurst 6 hatte hingegen einen niedrigen Bakteriengehalt. Die gewählte Nitratkonzentration hatte somit auf das LaktobazillenWachstum eine klare inhibitorische Wirkung, die besonders ausgeprägt beim Arbeiten mit ungewöhnlich geringen (keine Hemmwirkung) und ungewöhnlich hohen (deutliche Hemmwirkung) Nitratzusätzen zum Ausdruck kam.

Im Anfangsstadium des Versuchs kam es zu einer sehr raschen Nitritbildung; in den Würsten 4-6 stieg der Nitritgehalt auf über $100 \mathrm{ppm}$ (Bild 13), in der Wurst 6 blieb er während der gesamten Versuchsdauer auf einem hohen Niveau, und bei der Wurst 5 sank er auf die Hälfte seines Maximums.

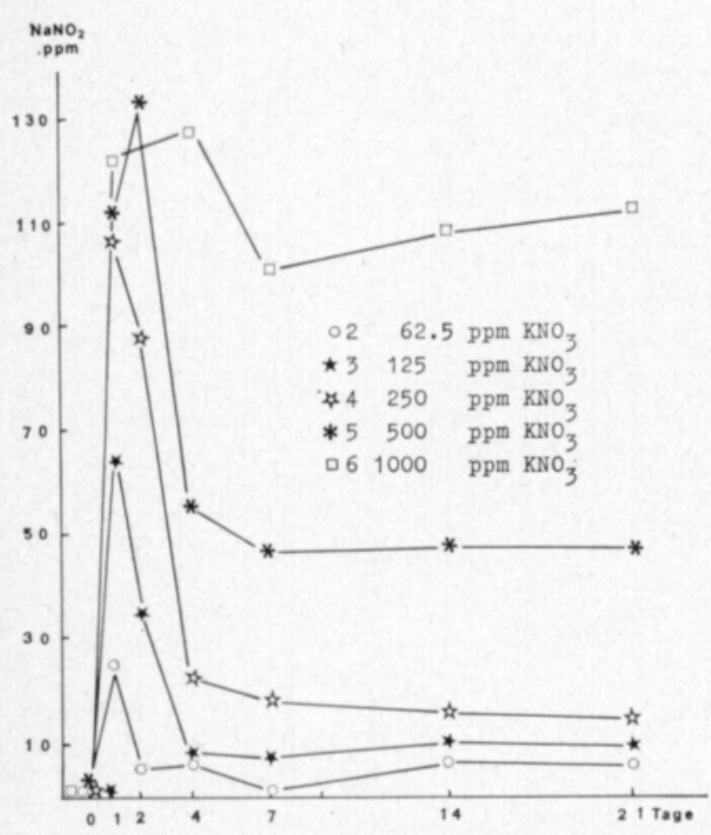

Bild 13. Einfluss der zugesetzten Nitratmenge auf den Restnitritgehalt. Versuchsreihe VII B.

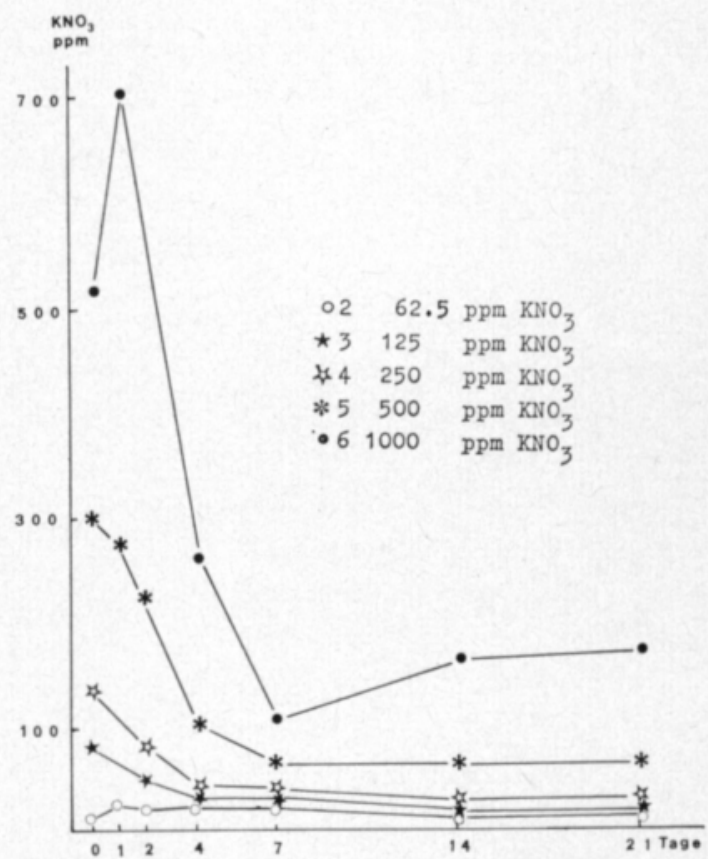

Bild 14. Einfluss der zugesetzten Nitratmenge auf den Restnitratgehalt. Versuchsreihe VII B.

Bei den übrigen Würsten sank der Nitritgehalt innerhalb der ersten zwei Tage auf ein Niveau von etwa $10 \mathrm{ppm}$ und blieb dann bis Versuchsende nahezu unverändert. Der Korrelationskoeffizient zwischen zugesetzter Nitratmenge und Restnitritmenge hatte einen Betrag von $\mathrm{r}=+0,991$.

In den ersten zwei bis drei Tagen war eine rasche Nitratreduktion zu verzeichnen, danach ging die Reduktionsgeschwindigkeit zurück (Bild 14). Die Restnitratmenge war direkt proportional zur zugesetzten Nitratmenge; der Korrelationskoeffizient betrug $\mathrm{r}=+0,986$.

Der Nitritschwund verlief nach einem schnellen Beginn sehr langsam, und zwar unabhängig vom $\mathrm{pH}$-Endwert. Nach dem vierten Tag nahm die 


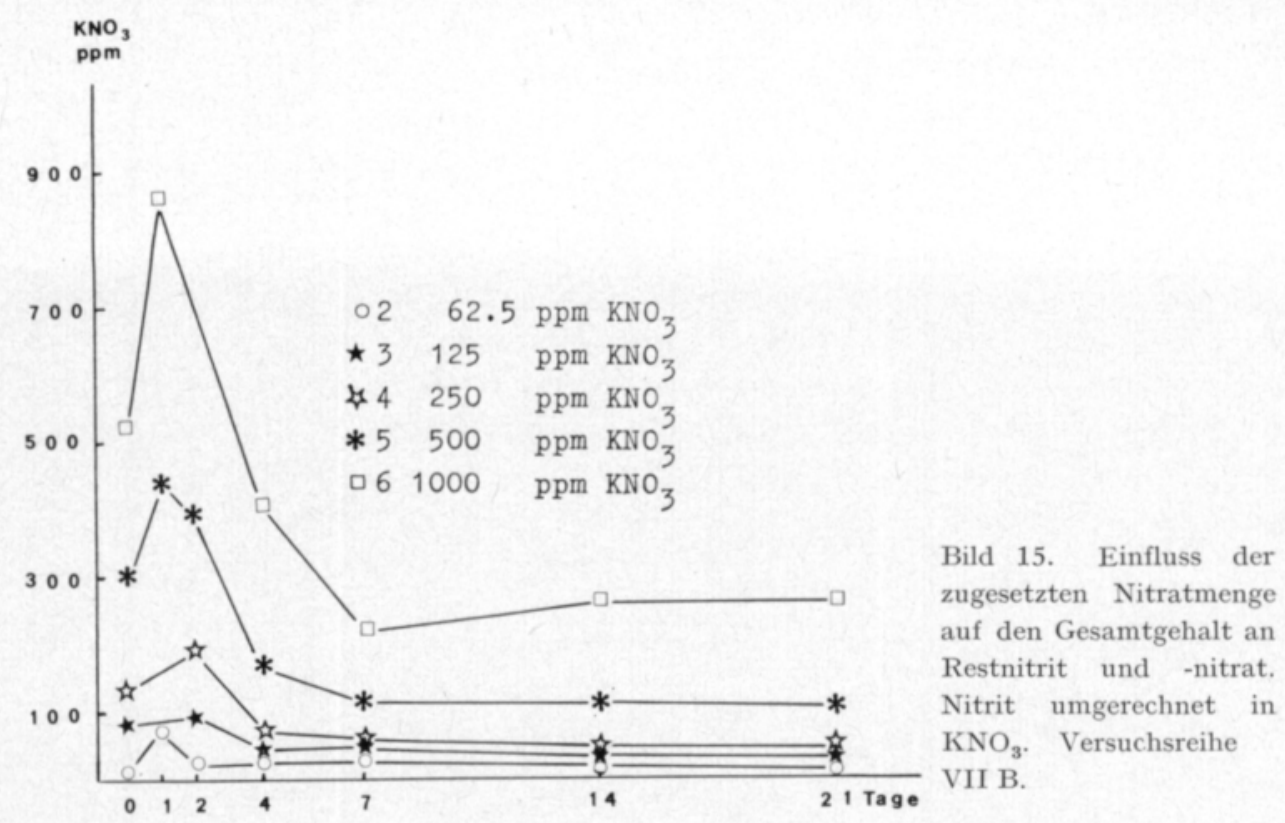

Gesamtmenge an Nitrit und Nitrat, als Nitrat gerechnet, nur in der Wurst 6 deutlich ab (Bild 15).

Im Versuch A erfolgte der $\mathrm{pH}$-Abfall bei den Würsten $3-6$ in gleicher Weise; wesentliche Unterschiede wurden nicht festgestellt (Bild 16). Bei der Wurst 1 sank der $\mathrm{pH}$ sehr rasch und auf einen niedrigen Wert, während er bei der Wurst 2 anfangs nur etwas schneller als bei den Würsten 3-6, dafür aber auch zwischen dem vierten und siebten Tag weiter abnahm und einen sehr niedrigen Endwert erreichte.

Im Versuch B zeigte die Wurst 1 die grösste $\mathrm{pH}-\mathrm{Abnahmegeschwindigkeit.}$ Bei der Wurst 2 verlief der pH-Abfall etwas rascher als bei den Würsten 3-6. Bei den $\mathrm{pH}$-Endwerten traten keine so grossen Differenzen wie im Versuch A auf, und die pH-Werte befanden sich zu Versuchsende in keiner Richtung in der den zugesetzten Nitratmengen entsprechenden Reihenfolge. Die pH-Endwerte lagen im Versuch B zwischen 4,85 und 5,04.

Eine Nitratzugabe von wenigstens $125 \mathrm{ppm}$ reichte somit aus, die $\mathrm{pH}$ Abfallgeschwindigkeit und den End-pH im normalen Bereiche zu halten, wobei das Ergebnis allerdings offensichtlich auch stark von der Eigenbakterienflora des verarbeiteten Fleisches mit beeinflusst wurde.

Bei den Würsten der Versuchsreihe VII wurde auch der NO-Myoglobingehalt (NOMb) bestimmt (Bild 17). Die Wurst 2 wies vor allem zu Versuchsende die niedrigste NOMb-Konzentration auf, jedoch war der Unterschied gegenüber den restlichen Würsten, die alle gleichgrossen NOMb-Gehalt hatten, gering. Die Wurst 2 hatte auch organoleptisch beurteilt eine hellere Farbe als die restlichen Würste.

Die Versuchsreihen II-VI hatten, was den Nitrit- und Nitratzusatz anbelangt, präliminaren Charakter und werden deshalb an dieser Stelle nicht näher behandelt. Das gleiche gilt für die Versuchsreihen IX und X, die hauptsächlich der Untersuchung des Askorbinsäure-Einflusses dienten. 


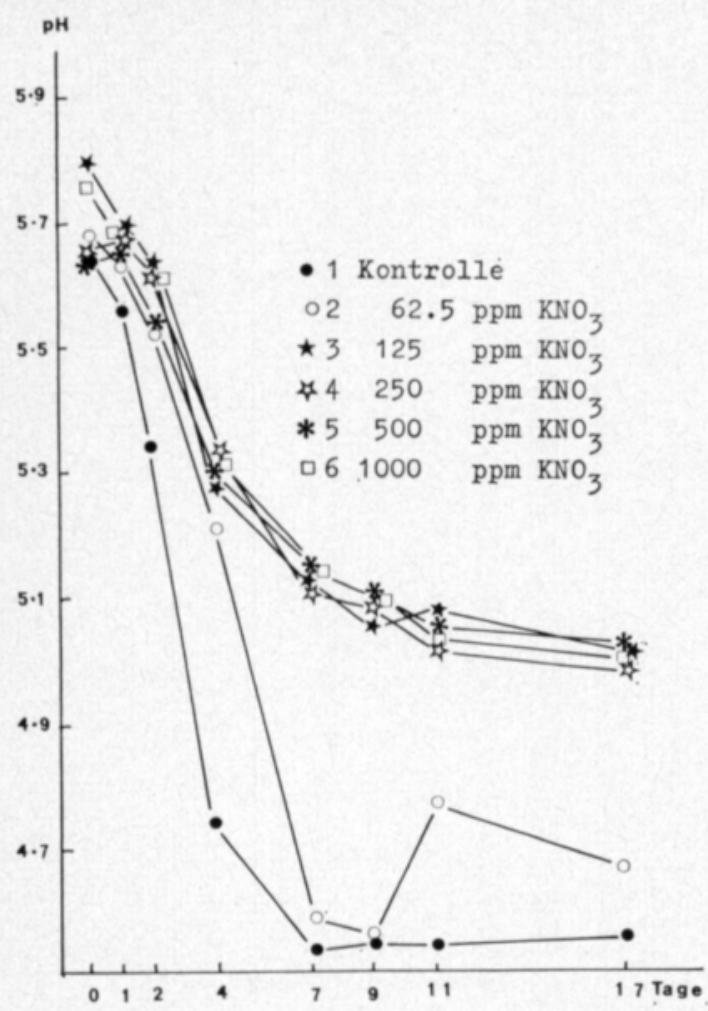

Bild 16. Einfluss derzugesetzten Nitratmenge auf den pH-Wert. Versuchsreihe VII A.

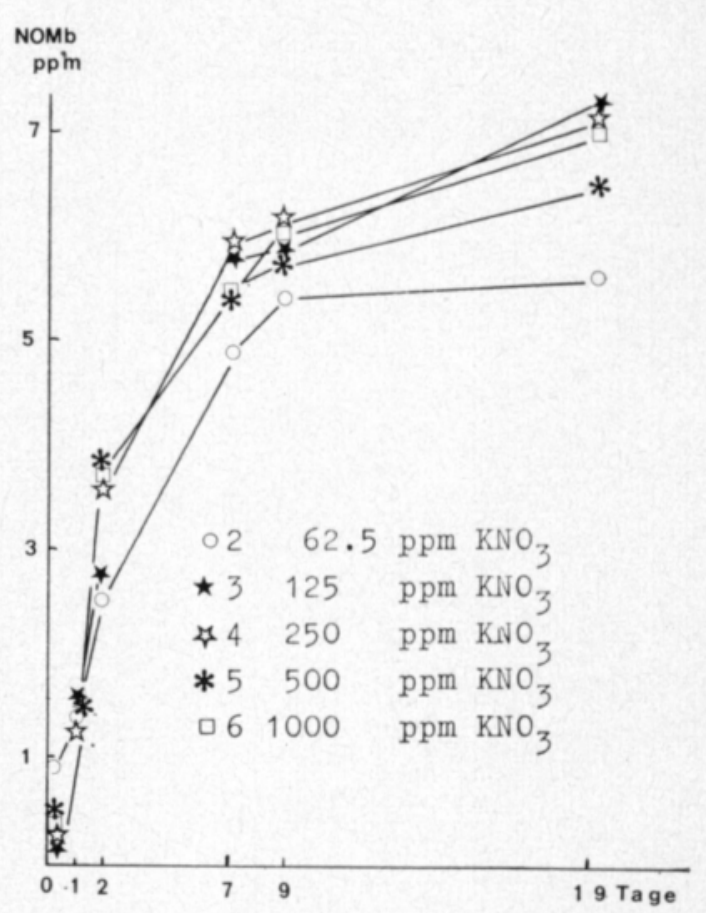

Bild 17. Einfluss der zugesetzten Nitratmenge auf den Stickoxidmyoglobin-Gehalt. Versuchsreihe VII $B$.

In der Versuchsreihe XIV wurde das Mengenverhältnis Nitrit/Nitrat so variiert, dass die Gesamtmenge, gerechnet als Nitrit-Äquivalent, konstant b li e b. Um eventuelle Differenzen deutlich sichtbar zu machen, wurde mit einer verhältnismässig grossen Nitrit-/Nitrat-Menge gearbeitet.

Der Versuch zeigte in augenfälliger Weise, dass bei Zusatz von Nitrat und Nitrit lediglich während der ersten vier Tage Unterschiede auftraten (Tabelle 10). Diese Analogie war also bei allen drei Faktoren (Nitrit, Nitrat und NitritNitrat-Gesamtmenge) zu beobachten. Am Herstellungstag entsprachen die Unterschiede ganz den Zusätzen; im Laufe der ersten 24 Stunden ging der Nitritgehalt der Würste 3-6 zurück, aber schon am zweiten Tag kam es offensichtlich infolge der Tätigkeit nitratreduzierender Bakterien - zu einem Stillstand der Nitritabnahme oder zu einem Anstieg der Nitritmenge. Die Nitratmenge stieg im Laufe des ersten Tages in den nitrithaltigen Würsten auch noch nach der ersten Analyse, was ein Anzeichen dafür ist, dass die Nitritoxydation nicht sofort vollständig erfolgte. Hinsichtlich der Nitrit-NitratGesamtmenge zeigten die einzelnen Würste keine Unterschiede.

Der pH-Wert lag bei der Kontrollwurst eindeutig niedriger als bei den übrigen Würsten. Es war eine allerdings sehr schwache Tendenz zu erkennen, 
Tabelle 10. Nitrit- und Nitratgehalte beim Arbeiten mit in unterschiedlichem Verhältnis zusammengesetzten Nitrit-Nitrat-Mischungen. Versuchsreihe XIV.

\begin{tabular}{|c|c|c|c|c|c|}
\hline \multicolumn{6}{|c|}{ Wurstposten ${ }^{1}$ ) } \\
\hline Zeit (Tage) & 2 & 3 & 4 & 5 & 6 \\
\hline \multicolumn{6}{|c|}{$\mathrm{NaNO}_{2}(\mathrm{ppm})$} \\
\hline 0 & 0,1 & 10,9 & 30,6 & 71,3 & 117,3 \\
\hline 1 & 7,0 & 19,3 & 24,0 & 57,6 & 94,1 \\
\hline 2 & 81,1 & 82,3 & 65,4 & 76,0 & 84,0 \\
\hline 4 & 60,1 & 60,1 & 49,4 & 57,0 & 50,8 \\
\hline 7 & 17,9 & 19,8 & 17,1 & 17,1 & 20,9 \\
\hline 9 & 21,1 & 20,7 & 18,3 & 19,3 & 17,6 \\
\hline 11 & 20,0 & 18,4 & 18,4 & 18,0 & 17,8 \\
\hline 22 & 15,4 & 16,1 & 14,2 & 13,7 & 16,3 \\
\hline \multicolumn{6}{|c|}{$\mathrm{KNO}_{3}(\mathrm{ppm})$} \\
\hline 0 & 214,7 & 188,7 & 172,8 & 124,6 & 71,6 \\
\hline 1 & 200,8 & 181,3 & 202,0 & 149,5 & 83,3 \\
\hline 2 & 60,3 & 83,2 & 97,3 & 81,3 & 66,5 \\
\hline 4 & 30,6 & 28,1 & 29,6 & 43,7 & 33,5 \\
\hline 7 & 35,2 & 33,4 & 32,8 & 28,9 & 39,1 \\
\hline 9 & 31,1 & 27,5 & 26,7 & 34,0 & 33,4 \\
\hline 11 & 24,5 & 24,9 & 25,6 & 26,9 & 25,6 \\
\hline 22 & 22,3 & 21,1 & 20,2 & 21,8 & 20,6 \\
\hline
\end{tabular}

1) Siehe Tabelle 4

dass bei Würsten, die zu Beginn weniger Nitrit enthielten, der pH-Wert schneller sank als bei Würsten, die zu Beginn mehr Nitrit enthielten. Die Unterschiede waren bescheidener Grössenordnung, so dass sie keinen ausschlaggebenden Einfluss hatten.

Nach Ablauf von 24 Stunden hatten mit Ausnahme der Kontrollwurst sämtliche Würste eine graue Oberfläche und einen roten Kern. Die Würste 5 und 6 zeigten an der Oberfläche deutlich einen grauen Ring. Am vierten Tag waren sämtliche Würste mit Ausnahme der Kontrollwurst durchgehend rot gefärbt. Bezüglich der Schnittflächenfarbe zeigten die, Würste keine grossen Unterschiede. Lediglich die Wurst 5 hatte in beiden Versuchen eine signifikant bessere Schnittflächenfarbe (Rangordnungsprüfung) als die übrigen Würste. Die Würste 4 und 6 zeigten gleichgute Schnittflächenfarbe, wohingegen die Oberflächenfarbe bei der Wurst 4 deutlich besser als bei den Würsten 5 und 6 war.

Hinsichtlich des Bakteriengehaltes wiesen die Würste der Versuchsreihe XIV im allgemeinen nur geringfügige quantitative Unterschiede auf; bei den höheren Nitritgehalten wurde die Vermehrung der Laktobazillen und damit auch die $\mathrm{pH}$-Abnahme etwas verzögert. Was die Farbgüte betrifft, erwiesen sich die Konzentrationen 50 ppm $\mathrm{NaNO}_{2}$ und 177 ppm $\mathrm{KNO}_{3}$ (Wurst 4) als günstigste Werte. 


\section{4.2 Starterkulturen und GdL}

Der Einfluss der Starterkulturen wurde in den Versuchsreihen III-V, XI und XII untersucht.

In den Versuchsreihen II - V w urden bei verminderten Nitratkonzentrationen Vergleiche zwischen Starterkulturen und GdL angestellt.

Allerdings ist zu berücksichtigen, dass sämtliche Würste MikrokokkenStarterkultur enthielten, was einen Ausgleich der Ergebnisse bewirkte. Anderenfalls hätte die Verwendung von Nitrat zusammen mit GdL ihrer Grundlage entbehrt (Nurmi 1966 b).

In Tabelle 11 sind für die Würste der Versuchsreihen III-V die Nitritkonzentration, der $\mathrm{pH}$ und die Konsistenz aus der letzten Bestimmung zusammengestellt. Wie aus den Tabellenwerten hervorgeht, bewirkte die zugesetzte GdL-Menge $(0,5 \%)$ keinen besonders starken $\mathrm{pH}$-Abfall, wohl aber die zugesetzten Laktobazillen.

Tabelle 11. Restnitritmenge, pH-Wert und Konsistenz der Würste der Versuchsreihen III-V am letzten Bestimmungstag.

\begin{tabular}{|c|c|c|c|c|c|}
\hline & Wurstposten ${ }^{1}$ ) & & Restnitrit (ppm) & pH-Wert & Konsistenz (kg) \\
\hline 200 & ppm $\mathrm{NaNO}_{2}+\mathrm{GdL}$ & ………......... & 18,5 & 5,06 & 5,4 \\
\hline 500 & - $\mathrm{KNO}_{3}+\mathrm{GdL}$ & …................ & 28,5 & 5,11 & 4,2 \\
\hline 500 & , $\mathrm{KNO}_{3}+\mathrm{Lb}$ & ……........... & 30,4 & 4,80 & 8,0 \\
\hline 100 & $\mathrm{ppm} \mathrm{NaNO}_{2}+\mathrm{GdL}$ & .................. & 10,5 & 5,13 & 4,7 \\
\hline 250 & $\mathrm{KNO}_{3}+\mathrm{GdL}$ & ..................... & 12,4 & 5,19 & 4,7 \\
\hline 250 & $\mathrm{KNO}_{3}+\mathrm{Lb}$ & ................... & 8,3 & 4,63 & 9,6 \\
\hline 50 & $\mathrm{ppm} \mathrm{NaNO}_{2}+\mathrm{GdL}$ & …............... & 7,6 & 4,98 & 4,4 \\
\hline 100 & - $\mathrm{KNO}_{3}+\mathrm{GdL}$ & .................. & 9,1 & 5,11 & 4,9 \\
\hline 100 & $\mathrm{KNO}_{3}+\mathrm{Lb}$ & ................. & 5,9 & 4,52 & 8,8 \\
\hline
\end{tabular}

1) Siehe Tabelle 4

Die mit GdL hergestellten Würste hatten bei den niedrigeren Nitratkonzentrationen einen höheren Restnitritgehalt; diesen grossen relativen Unterschieden dürfte jedoch keine praktische Bedeutung zukommen. Als wichtigster Einflussfaktor bezüglich der Restnitritmenge ist die zugesetzte Nitrit- und Nitratmenge zu nennen. Der Korrelationskoeffizient zwischen zugesetztem Nitrit und Restnitrit betrug bei den Nitrit-GdL-Würsten $r=+0,997$, zwischen zugesetztem Nitrat und Restnitrit bei den Nitrat-GdL-Würsten $r=+0,976$ und bei den Nitrat-Lb-Würsten $\mathrm{r}=+0,960$.

Die Würste wiesen ausnahmslos eine rote Farbe auf, wobei jedoch zwischen den GdL- und Lb-Würsten gewisse Unterschiede bestanden. Die GdL-Würste waren von Anfang an tiefrot mit einem Stich ins Bläuliche. Bei den Lb-Würsten vollzog sich die Umrötung langsamer, und ihre Schnittflächen hatten eine bräunliche Tönung. Letztere war um so stärker je mehr Nitrat zugesetzt worden war, verlor sich aber innerhalb von drei bis sieben Tagen. Bei sehr tiefem Absinken des $\mathrm{pH}$-Wertes, wie dies bei den Lb-Würsten mitunter der 
Fall war, verlor die Farbe an Intensität, blieb aber rot. Der oben umrissene Umrötungsablauf war sehr typisch für diese Würste und wiederholte sich in sämtlichen Versuchsreihen der Untersuchung. Der Umstand, dass die GdLWürste tiefrote Farbe mit einem Stich ins Bläuliche, die Lb-Würste dagegen ein deutlich helleres Rot aufwiesen, erschwerte namentlich die organoleptische Beurteilung der Umrötung in den einzelnen Stadien. In den Präliminarversuchen wurde denn auch ohne Hinzuziehung einer Beurteiler-Gruppe lediglich konstatiert, ob die Würste eine rote Schnitt- und Oberfläche hatten oder nicht. Allerdings wurde die Umrötung durch Farbaufnahmen dokumentiert.

In der Versuchsreihe XI wurde der Einfluss der Starterkultur-Konzentration a uf die Nitrit- und Nitratmengen und auf die Eigenschaften der Rohwurst untersucht. Der Umstand, dass dabei unbeabsichtigt mit ungewöhnlich hohen Bakteriengehalten (Tabelle 5) gearbeitet wurde, beeinträchtigt den praktischen Wert der Ergebnisse.

Bei den Nitrit-Würsten war die Starterkultur-Konzentration im Hinblick auf den Nitritgehalt ohne Belang. Dagegen zeigte sich bei den Nitrat-Würsten, dass die mit grösserem Starterkultur-Zusatz hergestellte Wurst ein höheres Nitritgehalts-Maximum und dieses schneller als Würste mit geringerem Starterkultur-Zusatz oder die Kontrollwurst erreichte (Bild 18).

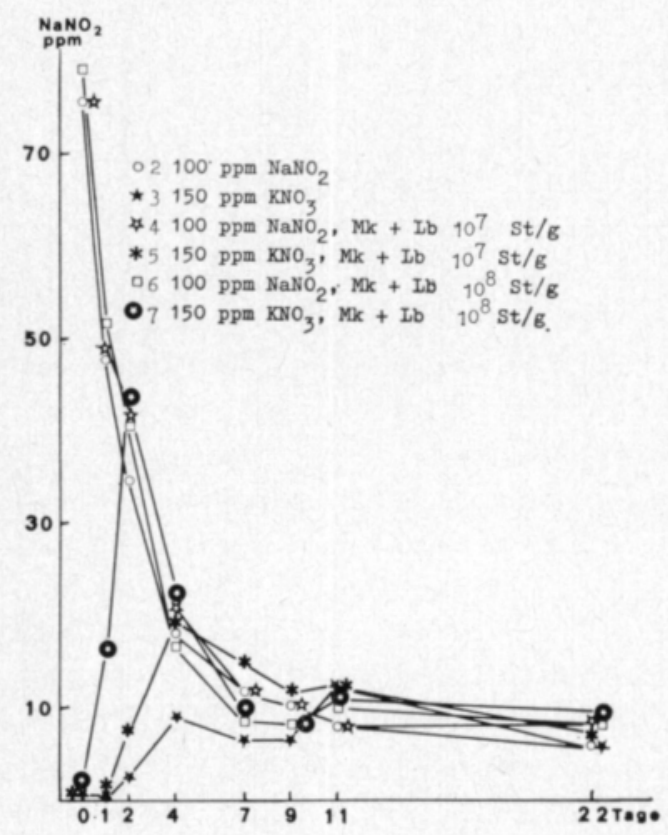

Bild 18. Einfluss der Starterkultur-Konzentration auf den Restnitritgehalt bei Einsatz von Nitrit oder Nitrat. Versuchsreihe XI.

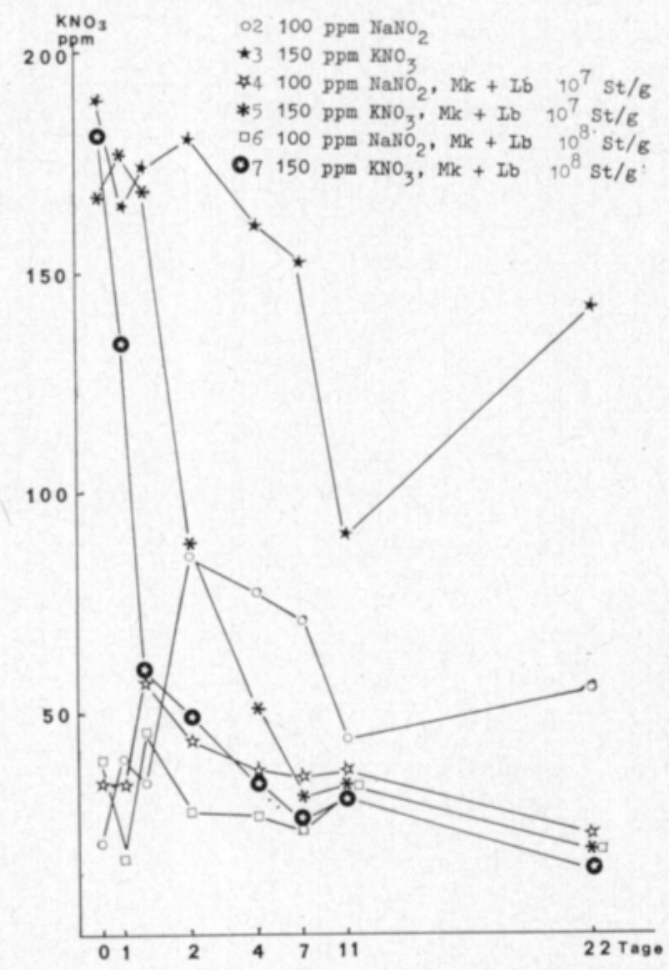

Bild 19. Einfluss der StarterkulturKonzentration auf den Restnitratgehalt bei Einsatz von Nitrit oder Nitrat. Versuchsreihe XII. 
Die Nitratmenge nahm bei den Nitrat-Würsten in der gleichen Reihenfolge $a b$, in der die zugesetzte Starterkultur-Menge stieg. Die Reduktion verlief am schnellsten in der Wurst 7, beinahe ebenso schnell in der Wurst 5 und am langsamsten in der Wurst 3 ; letztere hatte als einzige einen abweichenden Restnitratgehalt. Bei den Nitrit-Würsten hatte die Wurst 2 zu Versuchsende einen beträchtlich höheren Nitratgehalt als die Würste 4 und 6 (Bild 19).

Die Summe aus Nitrit- und Nitratgehalt lag bei der Wurst 3 in beiden Versuchen zu Versuchsende am höchsten; an zweiter Stelle lag im Versuch B die Wurst 2. Zwischen den Würsten 4-7 bestanden am Ende des Versuchs in dieser Beziehung keine wesentlichen Unterschiede.

Der pH-Abfall erfolgte am schnellsten bei den Würsten mit der höheren Starterkultur-Konzentration, deutlich langsamer hingegen bei den Würsten mit der geringeren Konzentration. Gleichzeitig wiederholte sich die Erscheinung: Je schneller der $\mathrm{pH}$-Abfall, desto niedriger der End-pH. Bei den NitratWürsten sank der $\mathrm{pH}$-Wert etwas schneller und tiefer als bei den Nitrit-Würsten. In beiden Versuchen hatte die ohne Starterkultur-Zusatz hergestellte NitritWurst einen höheren End-pH als die Kontrollwurst.

Nach Ablauf von 24 Stunden hatten die Nitrit-Würste einen roten Kern, jedoch ausnahmslos eine graue Oberfläche. Nach vier Tagen hatten sämtliche Würste eine rote Farbe, die mit zunehmender Starterkultur-Konzentration an Tiefe gewann, was insbesondere in einer Verbesserung der Oberflächenfarbe zum Ausdruck kam. Die Nitrat-Würste entwickelten die bessere Oberflächenfarbe. Nach elf Tagen hatten sich die Farbunterschiede ausgeglichen. Nach 22 Tagen hatten sämtliche Würste etwa gleiche Farbe; deutliche Unterschiede zwischen Nitrit- und Nitrat-Würsten und zwischen Würsten mit verschiedener Starterkultur-Konzentration waren nicht mehr festzustellen.

In der Versuchsreihe XII wurden Vergleiche zwischen den Einflüssen von vegetativen, tiefgekühlten und lyophilisierten Starterkulturen auf die Restnitrit- und -nitratmengen sowie auf die Eigenschaften der Rohwurst angestellt. Damit sollte in erster Linie geklärt werden, ob Tiefkühlen oder insbesondere Lyophilisieren die Aktivität der Starterkulturen, verglichen an der Vegetativform, beeinträchtigt.

Erschwert wurde der Vergleich in diesem Versuch dadurch, dass die tiefgekühlten und die gefriergetrockneten Starterkulturen zum einen und die Vegetativzellen zum anderen von zwei verschiedenen Züchtungs-Chargen stammten. Diese Verfahrensweise war insofern notwendig als man anderenfalls eine Úberalterung der Vegetativzellen hätte in Kauf nehmen müssen. Die Bakterienmengen der miteinander zu vergleichenden Würste waren deshalb nicht genau gleichgross (Tabelle 4). Die einen Tag alte Vegetativkultur wurde einschliesslich ihres Nährbodens direkt dem Brät zugesetzt.

Die einzelnen Behandlungsmethoden hatten keine grossen Differenzen im Nitritgehalt zur Folge (Bild 20). Allerdings wies die unter Zusatz von lyophilisierter Starterkultur hergestellte Wurst 2 den niedrigsten Nitritgehalt auf. Ein sehr augenfälliger Unterschied bestand hingegen am ersten Tag zwischen den Laktobazillen-Mikrokokken-Würsten $(5-7)$ und den Mikrokokken-Würsten $(2-4)$. Die Mikrokokken-Würste hatten nach drei Tagen 
einen beträchtlich höheren Nitritgehalt, nach sieben Tagen hatten sich jedoch dann die Unterschiede weitgehend ausgeglichen. Allerdings lagen die Nitritmengen bis zum Ende des Versuchs bei den Mikrokokken-Würsten höher als bei den Laktobazillen-Mikrokokken-Würsten. Der Grund für diesen Unterschied ist wahrscheinlich im $\mathrm{pH}$-Wert zu suchen, der bei den drei Tage alten Laktobazillen-Mikrokokken-Würsten im Versuch A im Mittel 0,7, im Versuch B (4 Tage) 0,3 Einheiten niedriger als bei den Mikrokokken-Würsten lag.

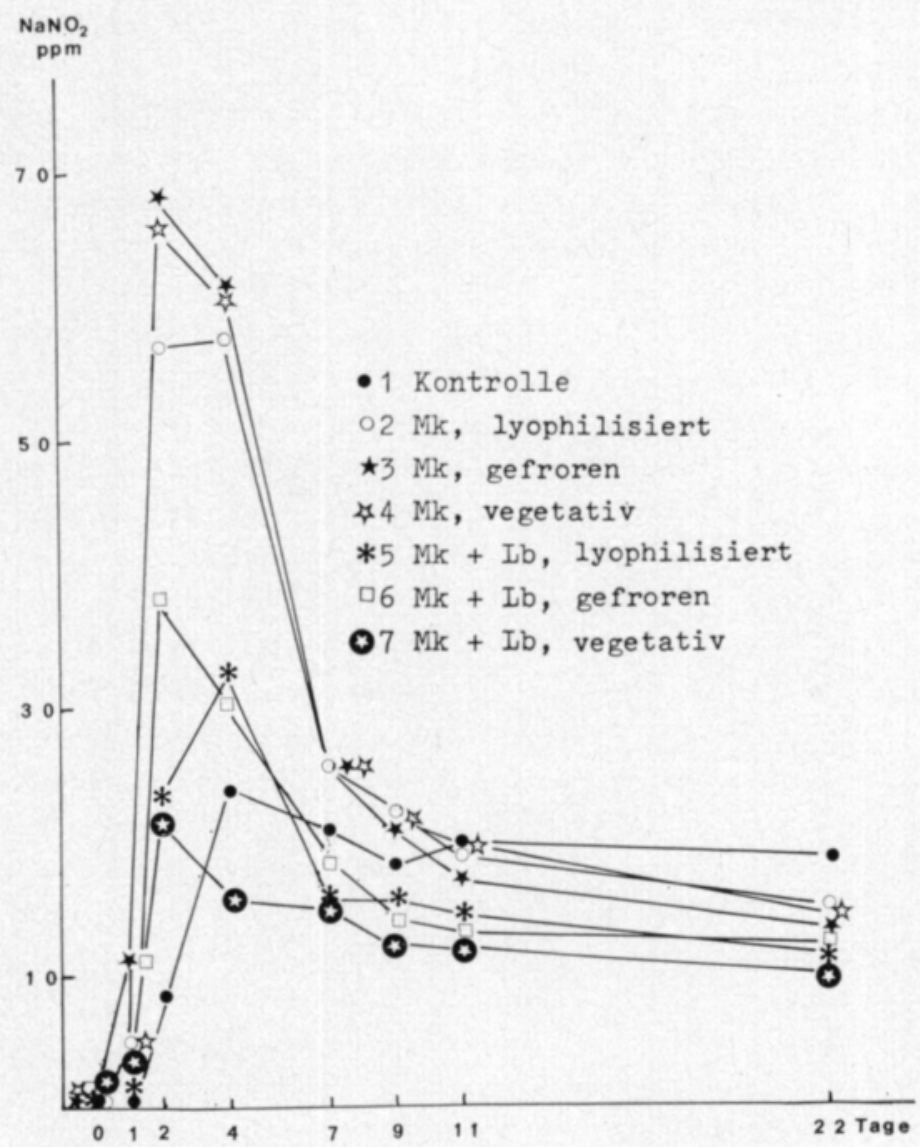

Bild 20. Einfluss von vegetativen, tiefgefrorenen und lyophilisierten Starterkulturen auf den Restnitritgehalt. Versuchsreihe XII.

Die Nitratgehaltsdifferenzen waren nicht bedeutend. Die Kontrollwurst hingegen enthielt vom vierten Tag an eindeutig mehr Nitrat.

Die besagten Differenzen waren auch aus den Nitrit-Nitrat-Gesamtmengenkurven ersichtlich. Die ausschliesslich mit Mikrokokken-Zusatz hergestellten Würste ergaben höhere Gesamtmengen als die Laktobazillen-Würste. Bei den Mikrokokken-Würsten wurde bei der Wurst 2 (gefriergetrocknete Starterkultur) eine höhere Gesamtmenge als bei den übrigen Würsten festgestellt.

Die pH-Wert-Änderungen schienen in diesem Versuch von der zugesetzten Bakterienmenge abhängig zu sein (Tabelle 4), wodurch die Interpretation der Ergebnisse erschwert wurde. Auf jeden Fall bewirkten die in Vegetativform zugesetzten Laktobazillen den schnellsten $\mathrm{pH}$-Abfall, wenngleich dabei zu berücksichtigen ist, dass es sich dabei um die mengenmässig stärkste Beimp- 
fung handelte. Die pH-Wert-Entwicklung der Mikrokokken-Würste entsprach der der Kontrollwurst.

Am dritten Tag wies die Kontrollwurst eine graue Oberflächenfarbe auf; die Schnittfläche zeigte eine breite rote Zone, der Kern war jedoch wieder grau. Zwischen den Würsten 2-4 (Mikrokokken-Würste) wurden in dieser Beziehung keine Unterschiede festgestellt; das gleiche trifft für die Würste 5-7 (Mikrokokken-Laktobazillen-Würste) zu. Die Mikrokokken-Würste zeigten deutlich einen Stich ins Bräunliche, und die sowohl Mikrokokken als auch Laktobazillen enthaltenden Würste hatten eine ansprechende rote Farbe. Zwischen dem siebten und elften Tag kam es zu einem Ausgleich der Farbtondifferenzen, jedoch hatten die Würste 5-7, verglichen an den Würsten 2-4, eine hellere, ja sogar zu helle Färbung. Am 22. Tag waren auch diese Unterschiede verschwunden; lediglich die Kontrollwurst hatte eine dunklere Farbe als die übrigen Würste.

\section{4.3 Askorbinverbindungen}

Der Einfluss von Askorbinverbindungen wurde in den Versuchsreihen I, $\mathrm{V}, \mathrm{VI}, \mathrm{IX}$ und $\mathrm{X}$ untersucht.

In der Versuchsreihe I wurden Vergleiche über den Einfluss verschiedener Askorbinverbindungen a uf die Rohwursteigenschaften angestellt. Bei diesen Verbindungen handelte es sich um Askorbinsäure, Natriumerythorbat, Natriumaskorbat und um eine Askorbinsäure-Natriumerythorbat-Mischung 1:1. Um eventuelle Unterschiede deutlich sichtbar zu machen wurden die Askorbinverbindungen in hohen Konzentrationen verwendet. Ausserdem wurden den Würsten Nitrat, GdL und Mikrokokken-Starterkultur zugesetzt. Dem GdL und der hohen Askorbinverbindungs-Konzentration war es offensichtlich zuzuschreiben, dass die Würste, was ihre mikrobiologischen, chemischen, physikalischen und auch ihre organoleptisch zu beurteilenden Eigenschaften betraf, keine wesentlichen Unterschiede aufwiesen. Sämtliche Würste zeigten eine gute Umrötung.

In den Versuchsreihen V und VI wurde der Einfluss der Askorbinsäure bei niedriger Nitrit- und Nitratkonzentration und gleichzeitiger Verwendung von Starterkulturen untersucht. In Tabelle 12 sind die Nitritgehalte der Würste nach drei Tagen und am Ende des Versuchs zusammengestellt.

Nach Tabelle 12 ergaben sich, wurde Askorbinsäure zugesetzt, kleinere Nitritgehalte als im gegenteiligen Fall. In dieser Phase der Untersuchung erfolgten die Bestimmungen ohne Einsatz von Quecksilberchlorid, welches die nitritzerstörende Wirkung der Askorbinsäure aufhebt, was sich durchaus in Form kleinerer Nitritgehaltswerte bei den askorbinsäurehaltigen Würsten niedergeschlagen haben könnte. Allerdings kommt diesen Unterschieden bei so geringen Restnitritmengen keine praktische Bedeutung zu.

Die Farbe der GdL-Würste war - sowohl bei als auch ohne Nitritzusatz bläulichrot. Die Laktobazillen-Würste wiederum wiesen eine bräunliche Tönung 
Tabelle 12. Restnitritmengen beim Arbeiten ohne bzw. mit Askorbinsäure.

\begin{tabular}{|c|c|c|c|}
\hline \multirow{2}{*}{$\begin{array}{l}\text { Wurstreihe: } \\
\text { Wurstposten }{ }^{3} \text { ) }\end{array}$} & \multicolumn{2}{|c|}{$\mathrm{NaNO}_{2}(\mathrm{ppm})$} & \multirow[t]{2}{*}{ Zugesetzte Mengen } \\
\hline & 3 Tage & 17 Tage & \\
\hline$(\mathrm{V}: 3)$ & $\left.9,5^{1}\right)$ & 5,9 & $100 \mathrm{ppm} \mathrm{KNO}_{3}$ \\
\hline$(\mathrm{V}: 4)$ & $\left.0,4^{1}\right)$ & 5,0 & $\left.\quad+\mathrm{AS}^{2}\right)$ \\
\hline (VI: 1$)$ & 2,2 & 5,3 & $34 \mathrm{ppm} \mathrm{NaNO}$ \\
\hline (VI: 2 ) & 0,3 & 2,2 & $+\mathrm{AS}$ \\
\hline (VI: 3 ) & 2,6 & 5,1 & $50 \mathrm{ppm} \mathrm{KNO}_{3}$ \\
\hline (VI: 4) & 0,4 & 2,6 & $+\mathrm{AS}$ \\
\hline
\end{tabular}

\footnotetext{
1) 4 Tage

2) $\mathrm{AS}=0.2 \%$ Askorbinsäure

3) Siehe Tabelle 4
}

auf, die bei den askorbinsäurehaltigen Würsten stärker ausgeprägt war. Aufs Versuchsende zu stellte sich ein Ausgleich der Farbunterschiede ein, und die bräunliche Tönung der Laktobazillen-Würste verschwand völlig. Im übrigen entsprachen die Umrötungsergebnisse etwa denen der Versuchsreihen IX und X.

In den Versuchsreihen IX und X wurde der Einfluss einer Askorbinverbindung bei verminderten und bei normalen Nitrit- und Nitratzusätzen unter$\mathrm{sucht}$. Als Askorbinverbindung diente Natriumerythorbat in der in der Industrie üblichen Konzentration $(0,05 \%)$.

In der Versuchsreihe IX bewirkte die Erhöhung des Nitritzusatzes von 25 ppm auf 200 ppm einen Rückgang der Gesamtbakterienzahl um durchschnittlich 1 logarithmische Einheit. Das Erythorbat bewirkte eine Abnahme der Gesamtbakterienzahl um durchschnittlich 0,3 logarithmische Einheiten. Für Laktobazillen lauteten die entsprechenden Werte 1,1 bzw. 0,4 logarithmische Einheiten.

Die Erhöhung der Nitritkonzentration hatte kaum Auswirkungen auf die Mikrokokken, wohl aber auf die fäkalen Streptokokken, die bei der höheren Nitritkonzentration zahlenmässig schwächer vertreten waren. Bei Zusatz von Starterkulturen nahm in der Kontrollwurst die Zahl der fäkalen Streptokokken um ein geringes ab; Erythorbat zeigte hingegen keine diesbezügliche Wirkung. Bezüglich der Hefemenge schien keinem der verwendeten Zusatzstoffe grosse Bedeutung zuzukommen. Lediglich die starterkulturfreie Kontrollwurst hatte einen etwas höheren Hefegehalt.

Der Nitritgehalt ging in sämtlichen Fällen sehr rasch zurück (Bild 21). Die erythorbathaltigen Würste enthielten eindeutig weniger Nitrit, was jedoch teilweise auf während der Analyse aufgetretene Verluste zurückzuführen sein dürfte. Der Nitratgehalt lag anfangs sehr hoch (Bild 22), in den erythorbathaltigen Würsten teilweise sogar höher als in der entsprechenden erythorbatfreien Wurst. Die Nitrit-Nitrat-Gesamtmenge blieb bei der niedrigeren Nitritkonzentration die ganze Zeit über nahezu konstant, wohingegen bei den Würsten mit der höheren Nitritkonzentration ein steiler Abfall zu beobachten war. Bei pH 5,0 zeigte der Nitritschwund eine Knickstelle. 


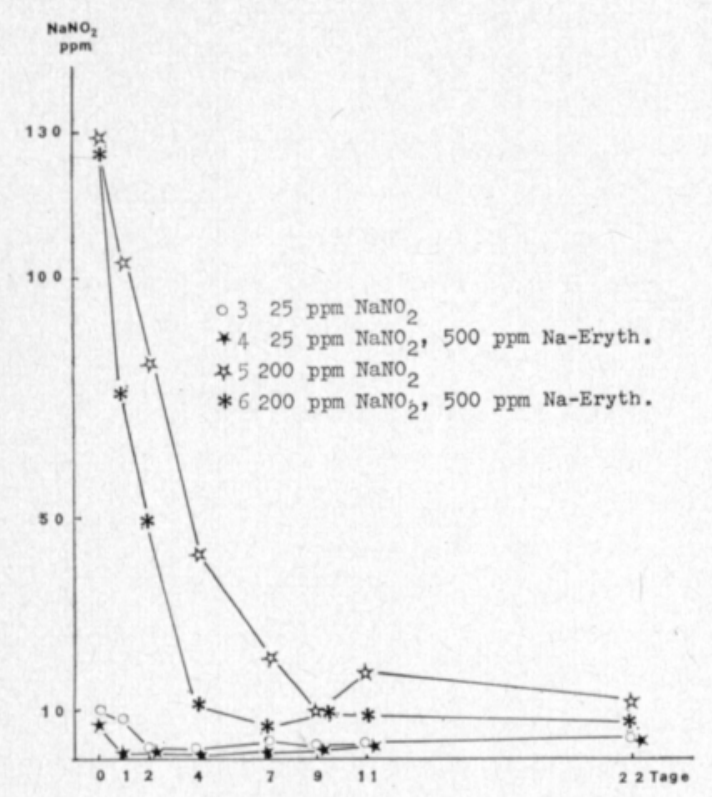

Bild 21. Einfluss des Erythorbats auf den Restnitritgehalt bei Zusatz von 25 bzw. 200 ppm $\mathrm{NaNO}_{2}$. Versuchsreihe IX.

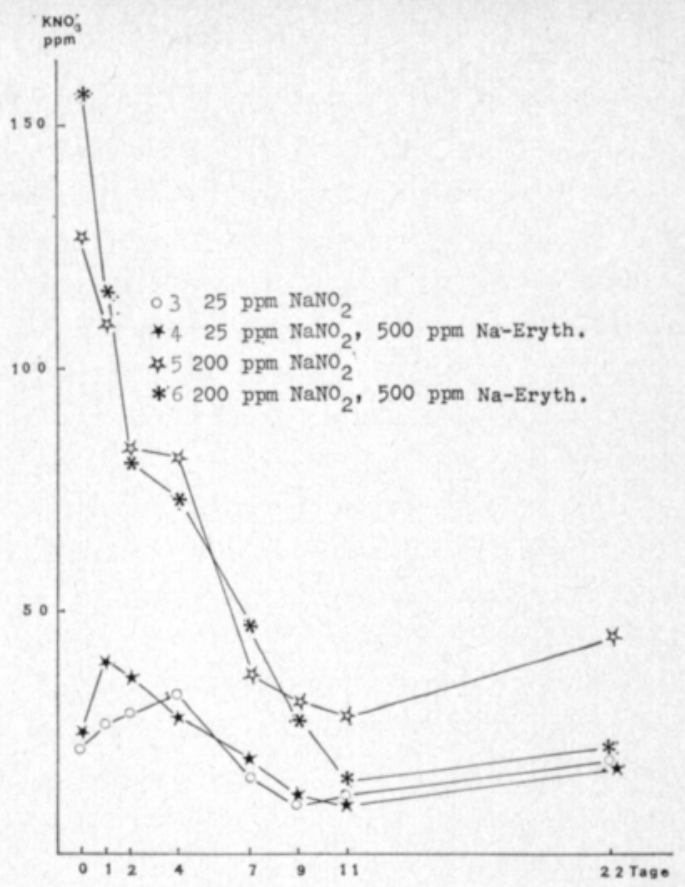

Bild 22. Einfluss des Erythorbats auf den Restnitratgehalt bei Zusatz von 25 bzw. 200 ppm $\mathrm{NaNO}_{2}$. Versuchsreihe IX.

Die pH-Entwicklung zeigte bei den einzelnen Nitritkonzentrationen einen unterschiedlichen Verlauf. Zwischen der nitritfreien Wurst 2 und den Würsten mit der niedrigeren Nitritkonzentration bestand kein Unterschied. In der starterkulturfreien Wurst nahm der $\mathrm{pH}$ langsam ab und blieb auf einem höheren Endwert als bei den übrigen Würsten. Die Erhöhung des Nitritzusatzes von $25 \mathrm{ppm}$ auf $200 \mathrm{ppm}$ hatte eine Zunahme des End-pH um 0,1$0,3 \mathrm{pH}$-Einheiten zur Folge.

Bei den erythorbathaltigen Würsten stellte sich bereits nach einem Tag eine rote Oberflächen- und Schnittflächenfarbe ein, während bei den erythorbatfreien Würsten hierzu zwei bis vier Tage erforderlich waren, ohne dass die gleiche Farbintensität erreicht wurde. Bei der niedrigeren Nitritkonzentration hatte die erythorbatfreie Wurst 3 eine mangelhafte Oberflächenfarbe. Nach elf Tagen wiesen alle nitrithaltigen Würste schlechte Oberflächenfarbe, aber rote Schnittflächenfarbe auf. Die ohne Erythorbatzusatz hergestellten Würste hatten vor allem was ihre Oberfläche betrifft eine etwas hellere Farbe.

Nach Ablauf von 22 Tagen hatte die Kontrollwurst braungraue Schnittflächenfarbe, während die übrigen Würste von satter Farbe waren. Die ohne Nitritzusatz hergestellte Starterkultur-Wurst hatte einen Stich ins Grünliche.

Etwa gleiche Ergebnisse wurden auch beim Arbeiten mit Nitrat (Versuchsreihe $\mathrm{X}$ ) erzielt; lediglich die Nitrit- und Nitratgehaltswerte weichen von den entsprechenden Resultaten der Versuchsreihe IX ab.

Der Nitritgehalt bewegte sich einen Tag lang sehr nahe bei null, jedoch kam es dann zwischen dem ersten und zweiten Tag zu einer zügigen Nitrat- 
reduktion. Nach zwei bis drei Tagen hatten die Würste 5 und 6 einen Nitritgehalt von 50-60 ppm, der jedoch dann zurückging. Bei den mit dem geringeren Nitratzusatz hergestellten Würsten blieb der Nitritgehalt die ganze Zeit über sehr niedrig. Erythorbat senkte den Nitritgehalt. Beim Nitratgehalt setzte nach dem ersten Tag eine sehr steile Abnahme ein, und die Reduktion erfolgte zum überwiegenden Teil zwischen dem ersten und vierten Tag. Bei den Würsten mit dem niedrigeren Nitratzusatz traten nur geringe Nitratgehaltsänderungen auf. Das Erythorbat hatte keinen Einfluss auf den Nitratgehalt.

Die Würste 4 und 6 hatten bereits nach einem Tag eine rote Oberfläche und auch eine durchweg rote Schnittflächenfarbe. Nach zwei Tagen waren alle nitrathaltigen Würste rot, jedoch zeigten die Würste 3 und 5 eine rotbraure Oberflächenfärbung. Nach vier Tagen hatten die Farben eine gewisse Ausgleichung und Dunklung erfahren. Die Hellfarbigkeit begann allmählich zu verschwinden, und nach elf Tagen waren die Farbunterschiede ausgeglichen, und die Würste hatten nun die Farbe ausgereifter Ware. Nach 22 Tagen war die Situation die gleiche. Die Wurstfarbe zeichnete sich durch ausserordentlich gute Licht- und Sauerstoffbeständigkeit aus. Das beste Resultat repräsentierte in dieser Beziehung die Wurst 6, gefolgt von der Wurst 5; die Würste 3 und 4 waren untereirander gleichwertig.

Die Kontıllwürste zeigten in frisch geschnittenem Zustand eine rote, wenn auch im Kern etwas grünliche Färbung, die jedoch dann unter der Luftund Lichteinwirkung in Graugrün umschlug.

\section{4.4 Zucker}

In der Versuchsreihe XVI wurde der Einfluss der Glukosekonzentration a uf dic Restnitritmenge und auf die Eigenschaften - in erster Linie auf den pH - der Rohwurst untersucht.

Bezüglich des pH-Wertes unterschieden sich die Würste deutlich voreinander (Bild 23). Die Wurst 1 hatte einen um etwa 0,2 Einhe.ten höheren $\mathrm{pH}$ als die Wurst 2, und diese wiederum einen um 0,2 Einheiten höheren $\mathrm{pH}$ als die Wurst 3. 0,1 Prozentpunkte Glukose bewirkten somit eire pH-Senkung um 0,1 Einheiten. Eine Ausnahme von dieser Regel machten die Würste 4 und 5.

Die Nitrit- und Nitratergebnisse waren unerwartet: Obgleich die $\mathrm{pH}$ Werte stark variierten, wiesen die Würste mit niedrigerem $\mathrm{pH}$ durchaus nicht weniger Nitrit bzw. Nitrat auf als die Würste, deren $\mathrm{pH}$ langsamer sank und auf einem höheren Wert stehenblieb. Die Farbe korrelierte mit dem pH-Wert in der Form, dass die Wurst 3, die den niedrigsten $\mathrm{pH}$ aufwies, die farbmässig beste war.

\subsubsection{Temperatur}

Die Versuchsreihe XII diente dem Zweck, den Einfluss der Temperatur im Anfangstadium der Herstellung auf die Eigenschaften von Rohwürsten 


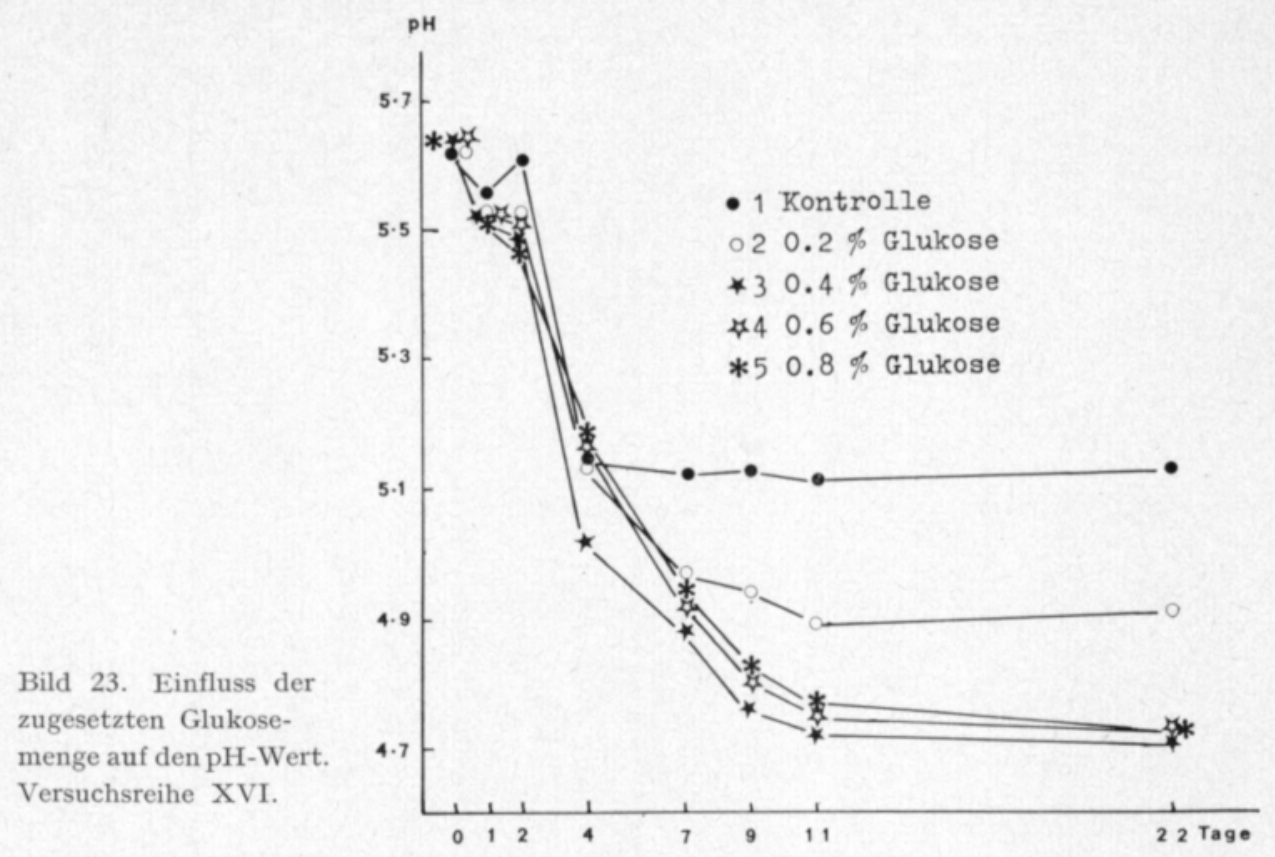

zu untersuchen, die entweder einen Nitrit-oder einen Nitratzusatz erhalten hat en.

Drei Tage vor Versuchsbeginn wurde das Flcisch in gefrorenem Zustand in einem grossen Kutter in Würfel von etwa $0,5-1 \mathrm{~cm}^{3}$ zerkleinert und sorgfältig gemischt. Von der so erhaltenen Masse wurde jeweils 16 Stunden vor der Herstellung einer Charge die dafür erforderliche Menge zum Auftauen ins Kühlhaus gebracht. Der Speck wurde stets dem gleichen Posten und direkt dem Gefrierhaus entnommen.

In Abständen von jeweils zwei Tagen wurden insgesamt fünfmal je zwei Wurstposten (in beiden 6 Würste) auf genau gleiche Weise hergestellt. Jedesmal wurde dem Wurstposten mit ungerader Ordnungszahl Nitrat, dem Wurstposten mit gerader Ordnungszahl Nitrit zugesetzt. Die Würste wurden zur gleichen Zeit in der gleichen Reifekammer gehalten; jedesmal wenn eine neue Wurstpartie in die Kammer gebracht wurde, wurde die Temperatur um $2^{\circ} \mathrm{C}$ gesenkt. Die Feuchte betrug ständig $95 \% \mathrm{rF}$. Am Morgen des zweiten Tages wurden die Würste leicht geräuchert, und zwar in der Form, dass alle Würste gleichoft Rauch erhielten. Die Temperaturprogramme sind in Tabelle 13 zusammengestellt.

Aus den Gesamtbakterienzahlen geht ziemlich deutlich hervor, dass am Ende des Versuchs die bei niedrigeren Temperaturen hergestellten Würste einen höheren Bakterienbesatz als die unter höheren Temperaturen hergestellten Würste aufwiesen (Bild 24). Allerdings gab es gewisse geringfügige Ausnahmen von diesem Trend, der sowohl bei den Nitrit- als auch bei den Nitrat-Würsten zu beobachten war.

Eine ähnliche Tendenz wurde bezüglich der Laktobazillenzahl festgestellt, und die Lag-Phase war bei den n'edrigeren Temperaturen länger (Bild 25). 
Tabelle 13. Die im Temperaturversuch (Versuchsreihe XIII) gefahrenen Temperatur- und Feuchtigkeitsprogramme (Nitrat-Würste mit ungerader, Nitrit-Würste mit gerader Ordnungszahl). Temperatur: ${ }^{\circ} \mathrm{C}$, Feuchtigkeit: $\%$ rF.

\begin{tabular}{|c|c|c|c|c|c|}
\hline $\begin{array}{l}\text { Wurst- } \\
\text { posten }^{1} \text { ) }\end{array}$ & $0-2$ & $2-4$ & $\begin{array}{c}\text { Zeit (Tage) } \\
4-6\end{array}$ & $6-7$ & $7-14$ \\
\hline 1,2 & $26^{\circ} / 95 \%$ rF & $24^{\circ} / 95 \%$ rF & $22^{\circ} / 95 \% \mathrm{rF}$ & $20^{\circ} / 95 \% \mathrm{rF}$ & $15^{\circ} / 80 \% \mathrm{rF}$ \\
\hline 3,5 & $24^{\circ} \%$ & $22^{\circ} \%$ & $20^{\circ} \%$ & $18^{\circ} \%$ & , \\
\hline 5,6 & $22^{\circ} \%$ & $20^{\circ} \%$ & $18^{\circ} /$ & $18^{\circ} \%$ & * \\
\hline 7,8 & $20^{\circ} \%$ & $18^{\circ} /$ & $18^{\circ} \%$ & $18^{\circ} \%$ & , \\
\hline 9,10 & $18^{\circ} \%$ & $18^{\circ} \%$ & $18^{\circ} \%$ & $18^{\circ} /$ & , \\
\hline
\end{tabular}

1) Siehe Tabelle 4

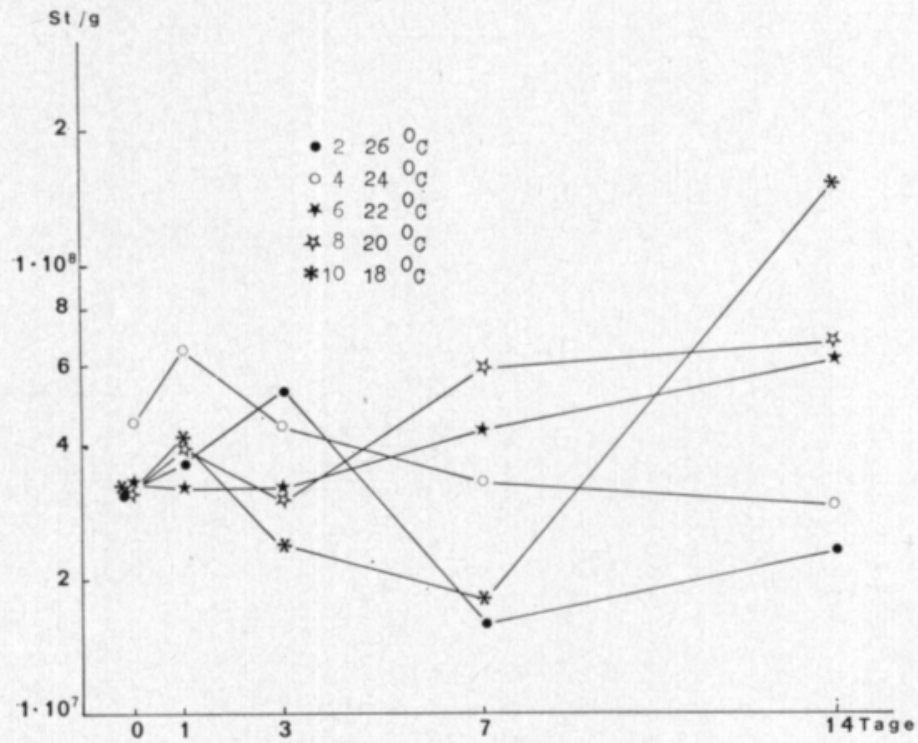

Bild 24. Einfluss der Reifetemperatur auf die Gesamtbakterienzahl. Nitrit-Würste, Versuchsreihe XIII.

Bei den Mikrokokkenzahlen traten auch hier keine wesentlichen Unterschiede auf, und auch die Nitrit- und Nitrat-Würste unterschieden sich nicht voneinander,

Da die Nitrit- und Nitrat-Resultate des Versuchs A in ihrer Entwicklung ziemliche Folgerichtigkeitsmängel erkennen lassen, stützen sich die folgenden Betrachtungen im wesentlichen auf den Versuch B. Bei den Nitritgehalten der Nitrat-Würste ist der Einfluss der Temperatur sehr deutlich zu erkennen (Bild 26). Die höchste Konzentration wurde bei der Wurst 1, die zweithöchste bei der Wurst 3 beobachtet. Die Wurst 5 erreichte ihr Maximum wahrscheinlich am zweiten Tag und lag zu diesem Zeitpunkt möglicherweise zwischen den Würsten 3 und 7; diese Annahme wird auch durch die am dritten Tag festgestellte Ergebnisreihenfolge gestützt.

Die flache Nitritgehaltskurve der Wurst 9 erreicht ihr Maximum am siebten Tage. Was die Nitrit-Würste betrifft, standen die Ergebnisse am dritten Tag in der Form in einer der Temperatur entsprechenden Reihenfolge, dass die 
Bild 25. Einfluss der Reifetemperaturauf die Laktobazillenzahl. Nitrit-Würste, Versuchsreihe XIII.
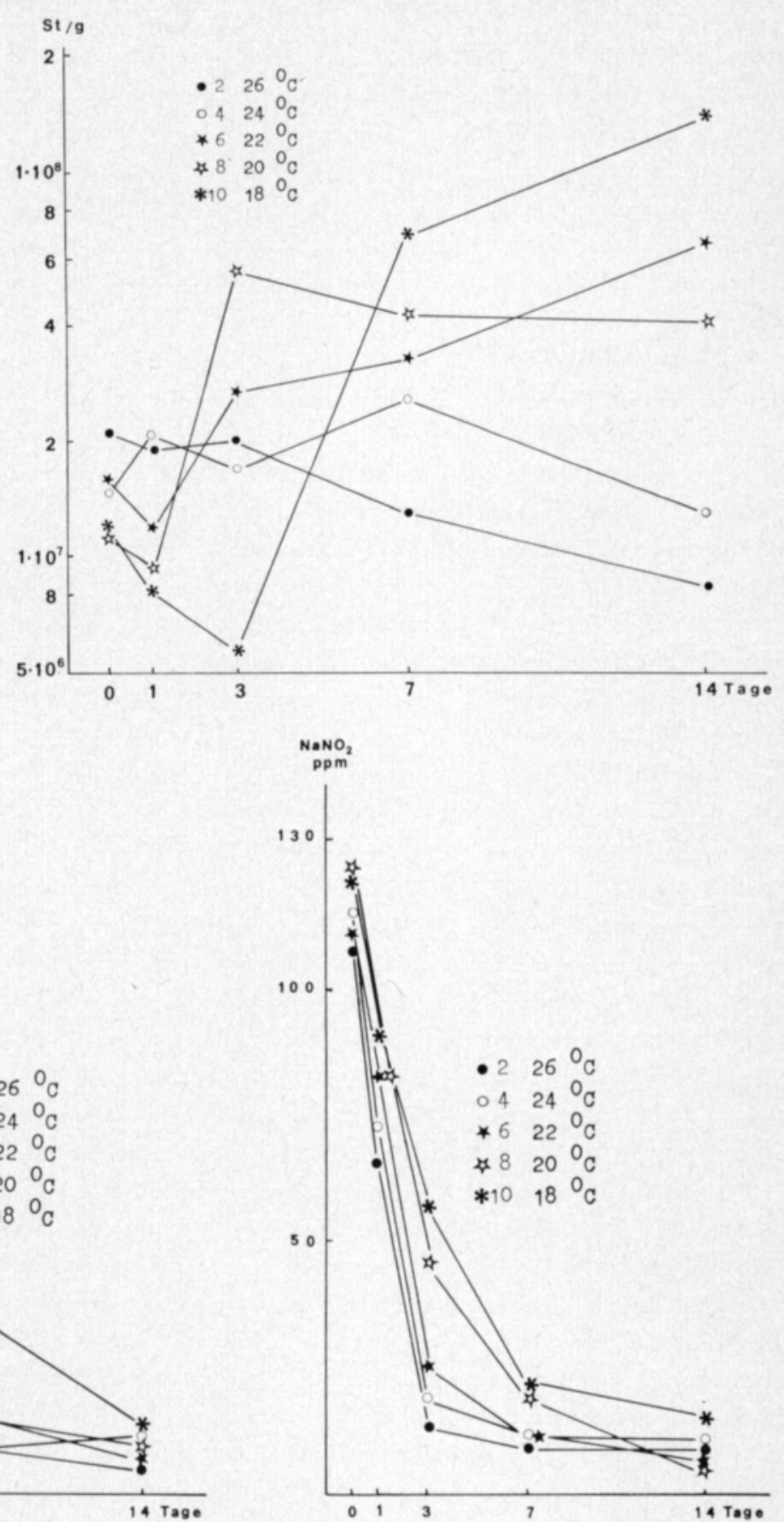

Bild 27. Einfluss der Reifetemperatur auf den Restnitritgehalt. Nitrit-Würste, Versuchsreihe XIII B.
Bild 26. Einfluss der Reifetemperatur auf den Restnitritgehalt. Nitrat-Würste,

Versuchsreihe XIII B.
- $126{ }^{0}$

$0324{ }^{\circ} \mathrm{C}$

* $522{ }^{\circ} \mathrm{C}$

\& $720{ }^{\circ}$

* $9180 \mathrm{C}$

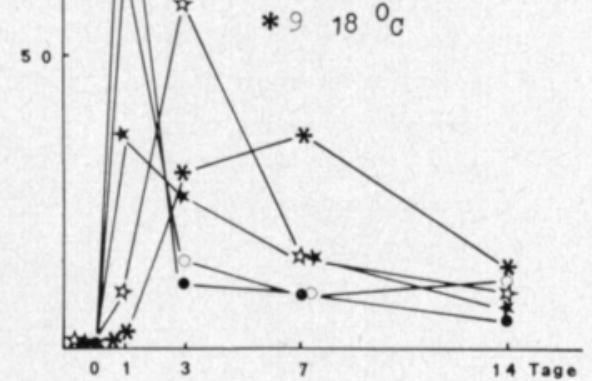

Wurst 2 den geringsten, die Wurst 10 den höchsten Nitritgehalt aufwies (Bild 27). Der höchste Restnitritgehalt wurde bei den Würsten 8 und 10 beobachtet, die Unterschiede zu und zwischen den übrigen Würsten waren jedoch so klein, 
dass sie ohne Bedeutung blieben. Bei den Nitrat-Würsten folgte der Nitratgehalt konsequent den Nitritgehaltsänderungen (Bild 28).

In den Nitrit-Würsten bildete sich bei den höheren Temperaturen weniger Nitrat; der maximale Nitratgehalt wurde bei $20^{\circ} \mathrm{C}$ erzielt. Die Nitrat-NitritGesamtmengen sowohl der Nitrit- als auch der Nitrat-Würste befanden sich nach drei bis sieben Tagen angenähert in einer dem Temperaturprogramm entsprechenden Reihenfolge; am Ende der Versuche wurden nur noch geringe und damit bedeutungslose Unterschiede festgestellt.

Bezüglich des pH-Werts schien die Temperatur die Würste in zwei Gruppen zu unterteilen: in die bei $26^{\circ}-22^{\circ} \mathrm{C}$ und die bei $20^{\circ}-18^{\circ} \mathrm{C}$ gereiften Würste (Bild 29 und 30).

Am schnellsten und auf den tiefsten Wert sank der $\mathrm{pH}$ bei den Würsten 1 und 2. Die pH-Wert-Kurven der Würste 3-6 kreuzten sich; wesentliche Unterschiede wurden in keiner Phase festgestellt. Bei den Würsten 7-10 ergaben sich etwas grössere, jedoch inkonsequente Differenzen: Im Versuch A war die Reihenfolge umgekehrt wie im Versuch B. In gewissem Grade unerwar tet - wenn auch übereinstimmend mit den mikrobiologischen Eıgebnissen - war, dass sich zwischen den Nitrit- und Nitrat-Würsten, die jeweils die gleiche Temperaturbehandlung erfahren hatten, keine Unterschiede zeigten.

Am ersten Tag waren bei der Versuchsreihe A zwischen den Würsten deutliche Farbunterschiede zu erkennen. Die Würste 1 und 2 waren sowohl an der Oberfläche als auch im Kern rot, die Würste 3 und 4 an der Oberfläche grau, im Kern rot, die Würste 5 und 6 an der Oberfläche grau, im Kern etwas weniger intensiv rot, die Würste 7 und 8 an der Oberfläche grau, im Kern graurot und die Würste 9 und 10 durchweg grau. Am dritten Tag hatten die Würste 1-6 eine intensive rote Oberflächen- und Schnittflächenfarbe, die Würste 7-10 eine graue Oberfläche und einen roten Kern. Nach sieben und 14 Tagen waren die Unterschiede verschwunden; lediglich bei den Würsten 7-10 zeigte sich am 14. Tag ein ganz leichter Grauton in der Oberflächenfarbe. Der Versuch B verlief etwas "langsamer»; am ersten Tag zeigten die Würste 3 und 4 an der Oberfläche, die Würste 5-10 an der Oberfläche und im Kern eine graurote oder graue Färbung. Am dritten Tag kam es zum Ausgleich der Farbdifferenzen. Auch am Ende des Versuchs wurden keine Unterschiede mehr festgestellt.

Der Vergleich zwischen Nitrit und Nitrat ergab in gewissem Grade widersprüchliche Resultate. Die bei den höheren Temperaturen unter Zusatz von Nitrit hergestellten Würste zeigten am ersten und dritten Tag vielleicht eine etwas bessere Schnittflächenfarbe, während die Nitrat-Würste durchweg eine bessere und hellere Oberflächenfarbe hatten. Am Ende des Versuchs wurde bei den Nitrat-Würsten eine geringfügig intensivere dunkelrote Färbung festgestellt. Die verschiedenen Temperaturen führten in dieser Hinsicht zu keinen Unterschieden.

Bei den höheren Temperaturen wurden also eine raschere Umrötung und ein beschleunigter Rückgang der Nitrit- und Nitratkonzentrationen und ein rascher Abfall des $\mathrm{pH}-$ Werts erzielt.

Zwischen Nitrit und Nitrat bestanden hingegen keine wesentlichen Unterschiede, höchstens dass mit Nitrat eine um eine Idee bessere Farbe erzielt wurde. 


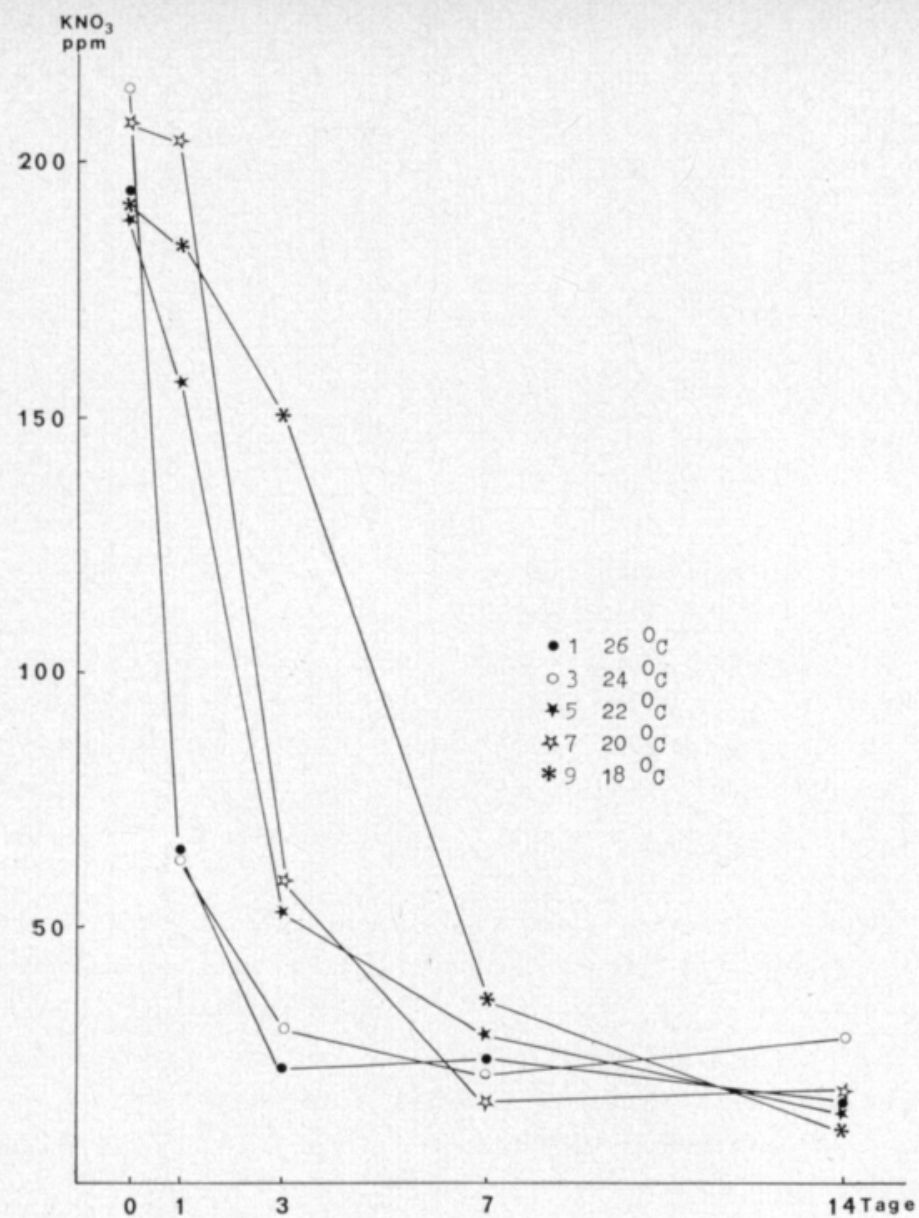

Bild 28. Einfluss der Reifetemperatur auf den Restnitratgehalt. Nitrat-Würste, Versuchsreihe XIII B.

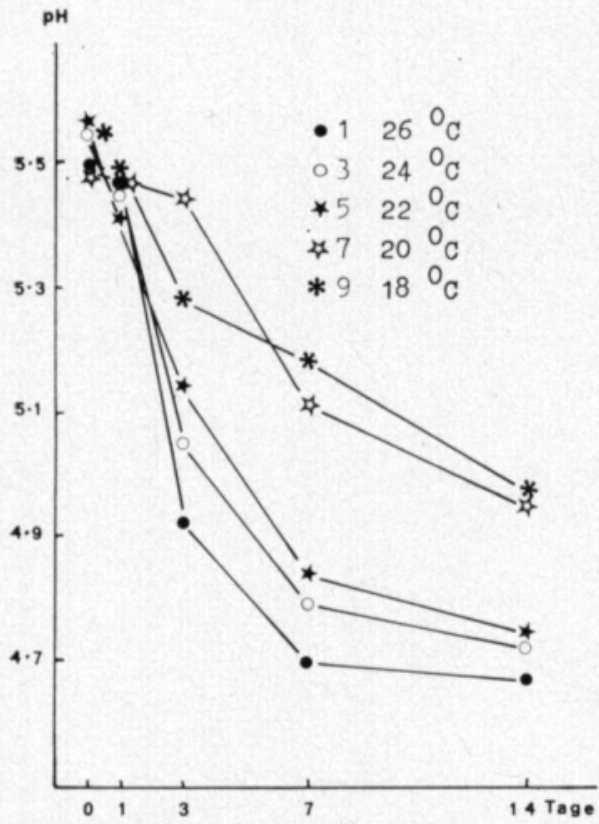

Bild 29. Einfluss der Reifetemperatur auf den pH-Wert. Nitrat-Würste, Versuchsreihe XIII.

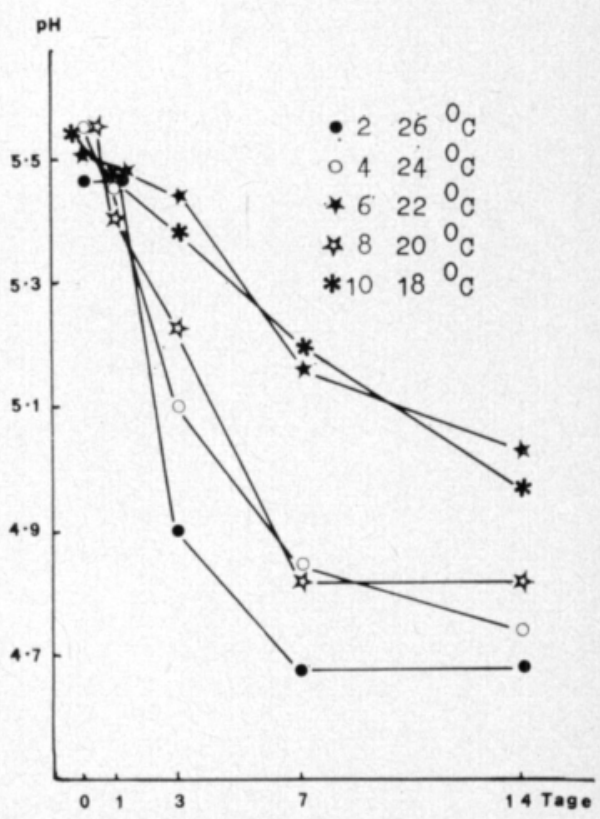

Bild 30. Einfluss der Reifetemperatur auf den pH-Wert. Nitrit-Würste, Versuchsreihe XIII. 
Der Einfluss der Temperatur kam in dieser Versuchsreihe recht deutlich zum Ausdruck, wenn er auch, der Praxis folgend, durch stufenweises Senken der Temperatur bis auf $18^{\circ} \mathrm{C}$ geschwächt worden war.

\section{4.6 Alter des Fleisches}

Mit der Versuchsreihe XV wurde bezweckt, den Einfluss des Fleischalters a uf die Nitratkonzentration und a uf die Eigenschaften der Rohwurst klarzustellen.

Für den Versuch lieferte der Schlachthof einen zwei Tage alten Schlachtkörper, von dem ein Vorderviertel abgetrennt und in einen Kühlraum von $2{ }^{\circ} \mathrm{C}$ und $95-100 \%$ rF gebracht wurde. Dem Viertel wurde sofort nach Eintreffen ein Fleischposten entnommen, nach einer Woche ein zweiter und nach abermals einer Woche ein dritter. Diese Fleischposten wurden jeweils sofort eingefroren und am Wurstherstellungstag alle gleichzeitig auf normale Weise aufgetaut. Der Versuch wurde zweimal - jeweils mit einem anderen Schlachtkörper - durchgeführt.

Die Gesamtbakterienzahlen lieferten kein vernünftiges Bild, denn obgleich die Wurst 3 in beiden Versuchen zu Beginn die höchste Bakterienzahl aufwies, verschwanden diese Unterschiede im Laufe des Versuchs. $\mathrm{Zu}$ Beginn wurden beim Versuch B zehnmal so viel Laktobazillen wie beim Versuch A festgestellt, und auch diese Unterschiede verschwanden im Laufe des Versuchs.

Zwischen den einzelnen Würsten bestanden zum Schluss Unterschiede in der Form, dass die Wurst 3 die meisten und die Wurst 1 die wenigsten Laktobazillen enthielt. Bei der Mikrokokkenzahl wurden keine in irgendeiner Weise regelmässigen Unterschiede festgestellt. Auch auf die Zahl der fäkalen Streptokokken schien das Fleischalter keinen Einfluss zu haben, wohl aber auf die Anzahl der Hefezellen, von denen zu Versuchsbeginn die Wurst 2 mehr als zehnmal so viel wie die Wurst 1, und die Wurst 3 etwa drei- bis fünfmal so viel wie die Wurst 2 enthielt. Mit Fortschreiten des Versuchs vermehrten sich die Hefen in den Würsten nicht, jedoch hatten die Würste 2 und 3 noch nach vier bis neun Tagen einen ziemlich hohen Gehalt an Hefezellen. Bei Versuchsende wurden nur noch ganz unbedeutende Differenzen festgestellt.

Weder die Nitritgehalts- noch die Nitratgehalts- noch die Nitrit-NitratGesamtmengenunterschiede zwischen den einzelnen Würsten zeigten irgendwelche Regelgebundenheit. Die Ergebnisse von Versuch A und Versuch B wichen in so hohem Grade voneinander ab, dass eine Beziehung zwischen Fleischalter und den Restmengen an Nitrit und Nitrat nicht festzustellen war.

Auch bei den $\mathrm{pH}$-Werten zeigten sich keine klaren Unterschiede. Lediglich im Versuch B hatte die Wurst 3 anfangs einen sehr hohen $\mathrm{pH}$, der jedoch bis zum neunten Tag abgenommen hatte.

Am ersten Tag hatte die Wurst 3 eine deutliche rötliche Färbung, die übrigen Würste hingegen nicht. Am zweiten Tag hatte die Wurst 3 eine kräftigere rote Oberflächenfarbe als die anderen (ohne Ring), wohingegen die Würste 1 und 2 im Kern ein intensiveres Rot zeigten. Nach vier Tagen war die Wurst 
1 sowohl innen als aussen kräftiger rot gefärbt als die Würste 2 und 3 , von denen wiederum die erstere röter als die letztere war.

\section{4.7 pH-Wert}

Die Bedeutung des pH-Wertes wurde in vorliegender Arbeit bisher bereits mehrmals gestreift. In diesem Abschnitt wird das betreffende Material noch einmal zusammenfassend dargestellt, denn nach Auffassung des Verfassers ist der $\mathrm{pH}$-Wert die wichtigste Einflussgrösse im Hinblick auf die Eigenschaften der Rohwurst; eine ganz andere Sache ist natürlich, welche Faktoren wiederum auf den $\mathrm{pH}$ einwirken.

Aus dem an Hand der Versuchsreihe VIII gezeichneten Diagramm (Bild 31) geht hervor, dass eine Erhöhung der Nitritkonzentration über den Wert 100 ppm hinaus die pH-Entwicklung nicht mehr wesentlich beeinflusst. Bei Konzentrationen unter $100 \mathrm{ppm}$ wiederum tendiert der $\mathrm{pH}$ zu zu starkem Abfall. Für Nitrat beträgt die entsprechende kritische Konzentration $\mathbf{1 2 5}$ ppm (Bild 32).

Der pH-Wert sinkt um so schneller und tiefer, je höhere Temperaturen gefahren werden (Bild 33 und 34). Hierbei ist zu berücksichtigen, dass bei der Versuchsreihe XIII die im Diagramm angegebene Temperatur nur zwei Tage gehalten wurde (s. Tabelle 13), so dass der Einfluss der Temperatur nicht so deutlich zum Ausdruck kommen konnte wie im Falle gleichbleibender Temperatur.

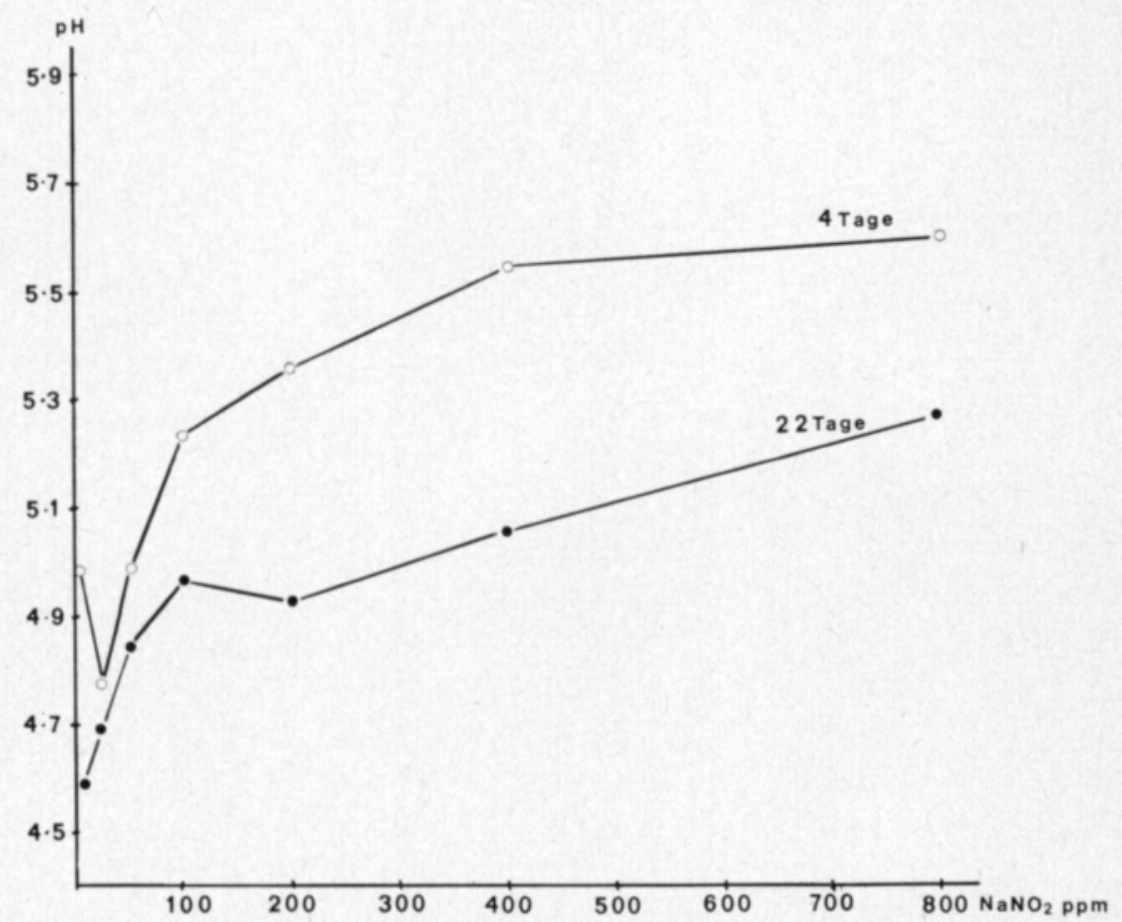

Bild 31. Einfluss der zugesetzten Nitritmenge auf den pH-Wert. Versuchsreihe VIII B. 


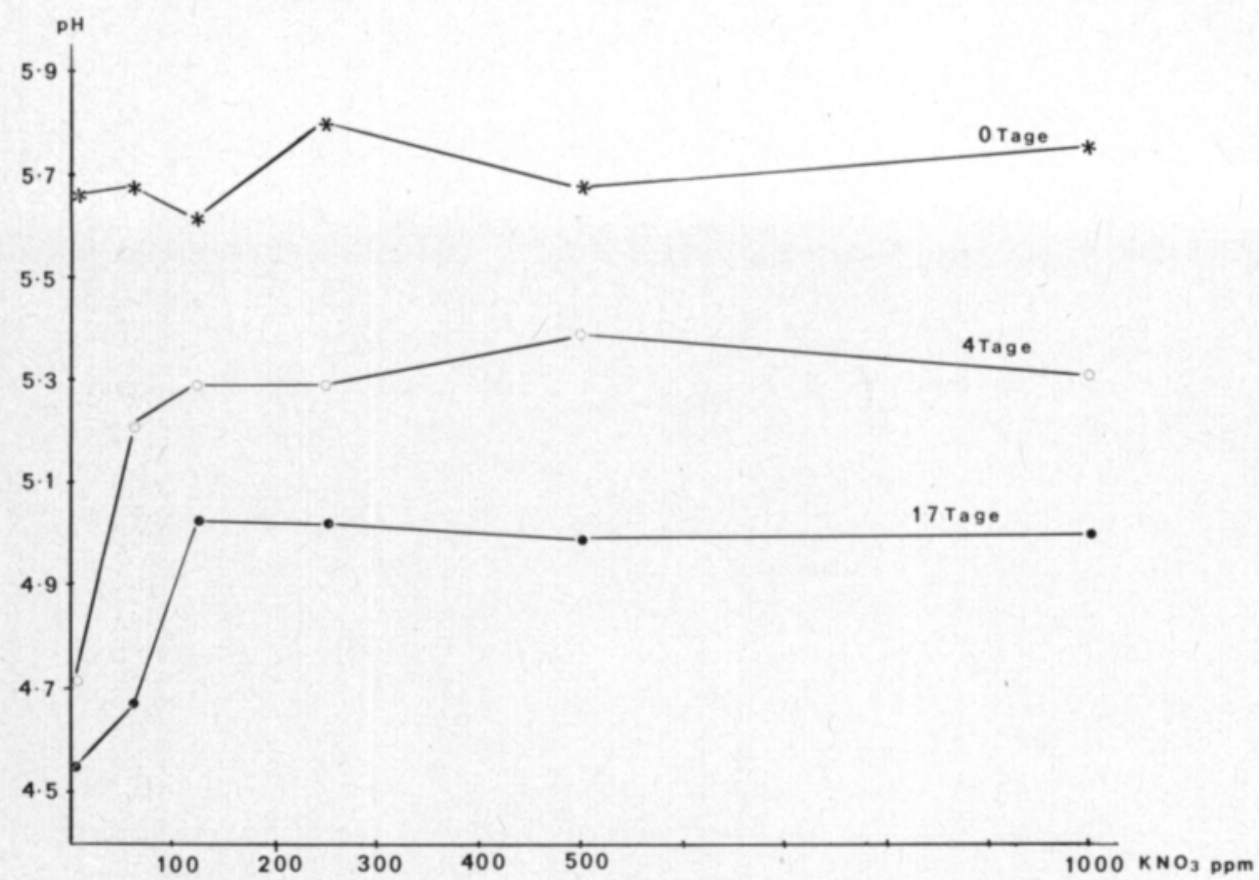

Bild 32. Einfluss der zugesetzten Nitratmenge auf den pH-Wert. Versuchsreihe VII.
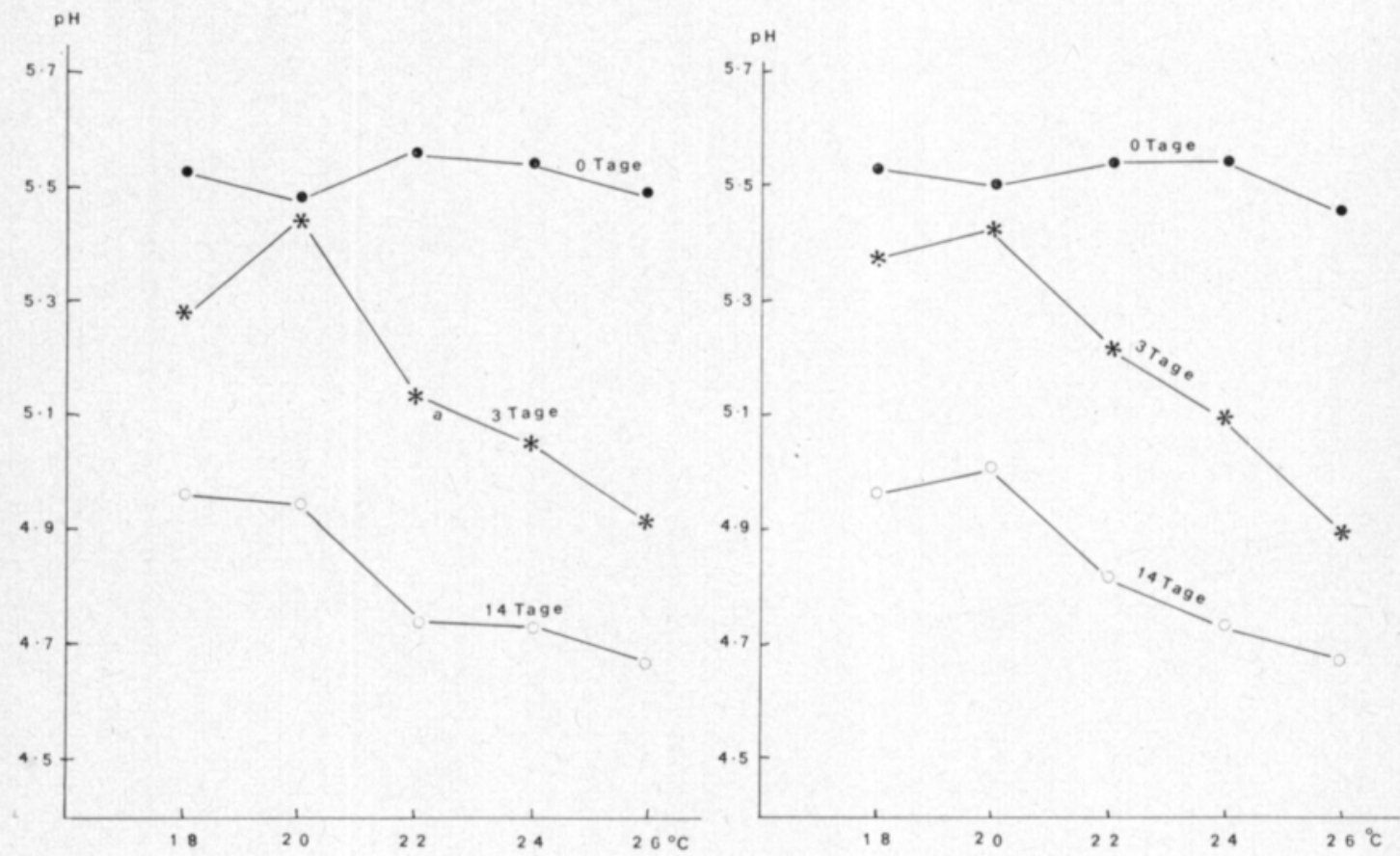

Bild 33. Einfluss der Reifetemperatur Bild 34. Einfluss der Reifetemperatur auf den auf den pH-Wert. Nitrit-Würste, Versuchsreihe XIII. pH-Wert. Nitrat-Würste, Versuchsreihe XIII. 
Bild 35. Einfluss der zugesetzten Glukosemenge auf den pH-Wert. Versuchsreihe XVI.

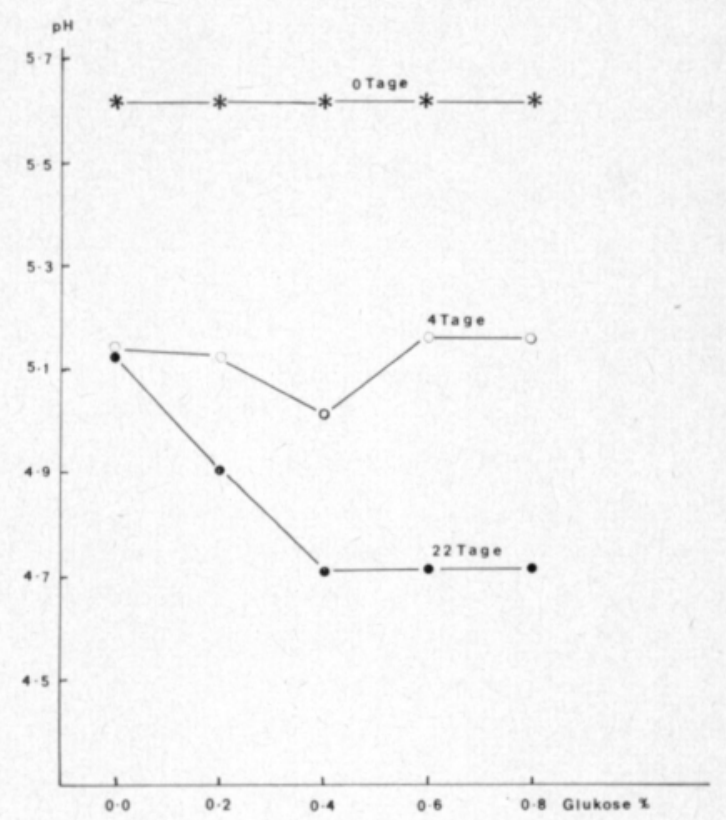

Im Versuch B lag der pH-Wert bei $20^{\circ} \mathrm{C}$ deutlich höher als bei $18^{\circ} \mathrm{C}$, was offensichtlich auf einen Materialfehler zurückzuführen war. Dadurch erhielt die Kurve der Mittelwerte eine unlogische Richtungsänderung nach oben.

Eine Erhöhung der Glukosekonzentration um 0,1\% bewirkte eine Senkung des End-pH um 0,1 Einheiten - dies jedoch nur bis zu einem Glukosegehalt von $0,4 \%$; danach war mit steigendem Glukosegehalt kein weiterer pHAbfall zu verzeichnen (Bild 35). Betrachtet man die am vierten Tag ermittelten Werte, so scheinen die höheren Glukosegehalte $(0,6$ und $0,8 \%)$ sogar eine Verzögerung des $\mathrm{pH}$-Abfalls bewirkt zu haben.

Bei den GdL-haltigen Würsten nahm der $\mathrm{pH}$ sehr schnell ab, blieb jedoch oberhalb des Wertes 5,0. Der End-pH wurde bereits nach vier Tagen erreicht; der Nitrit- bzw. Nitratgehalt war in dieser Beziehung ohne Bedeutung. Bei den entsprechenden Laktobazillen-Würsten setzte erst nach drei Tagen ein steiler $\mathrm{pH}$-Wert-Abfall ein, und ihr End-pH lag um 0,6-0,3 Einheiten niedriger als bei den entsprechenden GdL-Würsten. Eine Erhöhung der Nitratkonzentration von $100 \mathrm{ppm}$ auf $500 \mathrm{ppm}$ hatte einen Anstieg des End-pH um 0,3 Einheiten zur Folge (Tabelle 11).

\section{5 Ergebnisse der vergleichenden Versuche}

Auf Grund der Versuchsreihen VIII und VII wurde festgestellt, dass mit einem Nitritzusatz von $100 \mathrm{ppm}$ bzw. einem Nitratzusatz von $125 \mathrm{ppm}$ ein technologisch akzeptables Produkt erzielt wird. Die Resultate der Versuchsreihen V und VI zeigten, dass diese Feststellung auch beim Arbeiten mit GdL zutrifft. Nach den Ergebnissen der Versuchsreihe XIV ist es auch von Vorteil, eine Nitrit-Nitrat-Mischung zu verwenden. 
In den vergleichenden Versuchen wurden ausschliesslich die nitrithaltigen Würste unter Zusatz von GdL hergestellt. Die Nitritzugabe betrug $100 \mathrm{bzw}$. $200 \mathrm{ppm} \mathrm{NaNO}_{2}$. Die Herstellung der nitrathaltigen Würste erfolgte unter Einsatz von Mikrokokken-Laktobazillen-Starterkulturen. Der Nitratzusatz betrug 150 bzw. $300 \mathrm{ppm} \mathrm{KNO}_{3}$, was, umgerechnet in Nitrit, etwa den Nitritkonzentrationen der o.g. GdL-Würste entsprach. Weiter wurde Wurst mit $50 \mathrm{ppm} \mathrm{NaNO}_{2}$ und $75 \mathrm{ppm} \mathrm{KNO}_{3}$ entsprechend einer Nitritkonzentration von $100 \mathrm{ppm}$ hergestellt. Die Wahl der Zusatzmittelmengen wurde zum einen durch die Ergebnisse der Präliminarversuche, zum anderen durch entsprechende Angaben in der Fachliteratur (Leistner u.a. 1973 a und b, Wirth 1973, SKJELKVÅLE u.a. 1974) mitbestimmt. Auf Grund der Literaturauswertung und der Versuchsreihe XI wurde festgestellt, dass Voraussetzung für die Senkung des Nitrit- oder Nitratzusatzes die Verwendung von GdL oder Starterkulturen ist. Darum wurde in die vergleichenden Versuche keine Versuchsreihe ohne obengenannte Zusätze aufgenommen.

In der vorliegenden Arbeit wurde kein eigentlicher Vergleich zwischen Starterkultur- und GdL-Einsatz angestrebt; das Hauptgewicht lag vielmehr auf der Untersuchung der Auswirkungen einer Verringerung des Nitrit- bzw. Nitratzusatzes.

\subsubsection{Chemische Zusammensetzung}

Um eine gleichmässige Zusammensetzung der Versuchswürste zu gewährleisten wurden sie auf Wasser-, Fett- und Salzgehalt analysiert. Die Mittelwerte sämtlicher Proben sind in Tabelle 14 zusammengestellt; wie daraus ersichtlich ist, waren die Proben bezüglich ihrer chemischen Grundzusammensetzung, was die Mittelwerte betrifft, weitgehend homogen, wohingegen zwischen den Einzelwürsten mitunter ziemlich grosse Unterschiede auftraten, die jedoch nach Auffassung des Verfassers keinen signifikanten Einfluss auf die übrigen Ergebnisse hatten.

\section{5.2 Nitrit- und Nitratgehalt}

Der Nitritgehalt ist der zentrale Faktor dieser Studie. In Tabelle 15 und Bild 36 ist die Entwicklung des Nitritgehalts bei den Würsten der vergleichenden Versuchsreihen im Laufe des Herstellungsprozesses dargestellt. Bei den GdL-Würsten war schon am ersten Tag ein rascher Rückgang des Nitritgehalts zu beobachten. Die Wurst 1 mit der niedrigeren Konzentration enthielt am dritten und siebten Tag knapp halb so viel Nitrit wie die Wurst 2 mit der höheren Konzentration, am 14. Tag aber schon $2 / 3$ der in der Wurst 2 enthaltenen Menge.

Bei den Starterkultur-Würsten lag der Nitritgehalt nach Ablauf des ersten Tages sehr niedrig; dies traf auch für die mit einem Nitritzusatz von $50 \mathrm{ppm}$ hergestellte Wurst $5 \mathrm{zu}$. Nach drei Tagen erreichte die Nitritkonzentration ihr Maximum und begann dann langsam zu sinken.

Am dritten Tag hatte die Wurst 1 den signifikant kleinsten, die Wurst 4 den signifikant grössten Nitritgehalt. Ausserdem enthielt die Wurst $\mathbf{5}$ signifikant weniger Nitrit als die Wurst 2. Nach 14 Tagen hatte die Wurst 2 die 
Tabelle 14. Wasser-, Fett- und Salzgehalt der Würste der vergleichenden Versuchsreihen V1-V6, (\%).

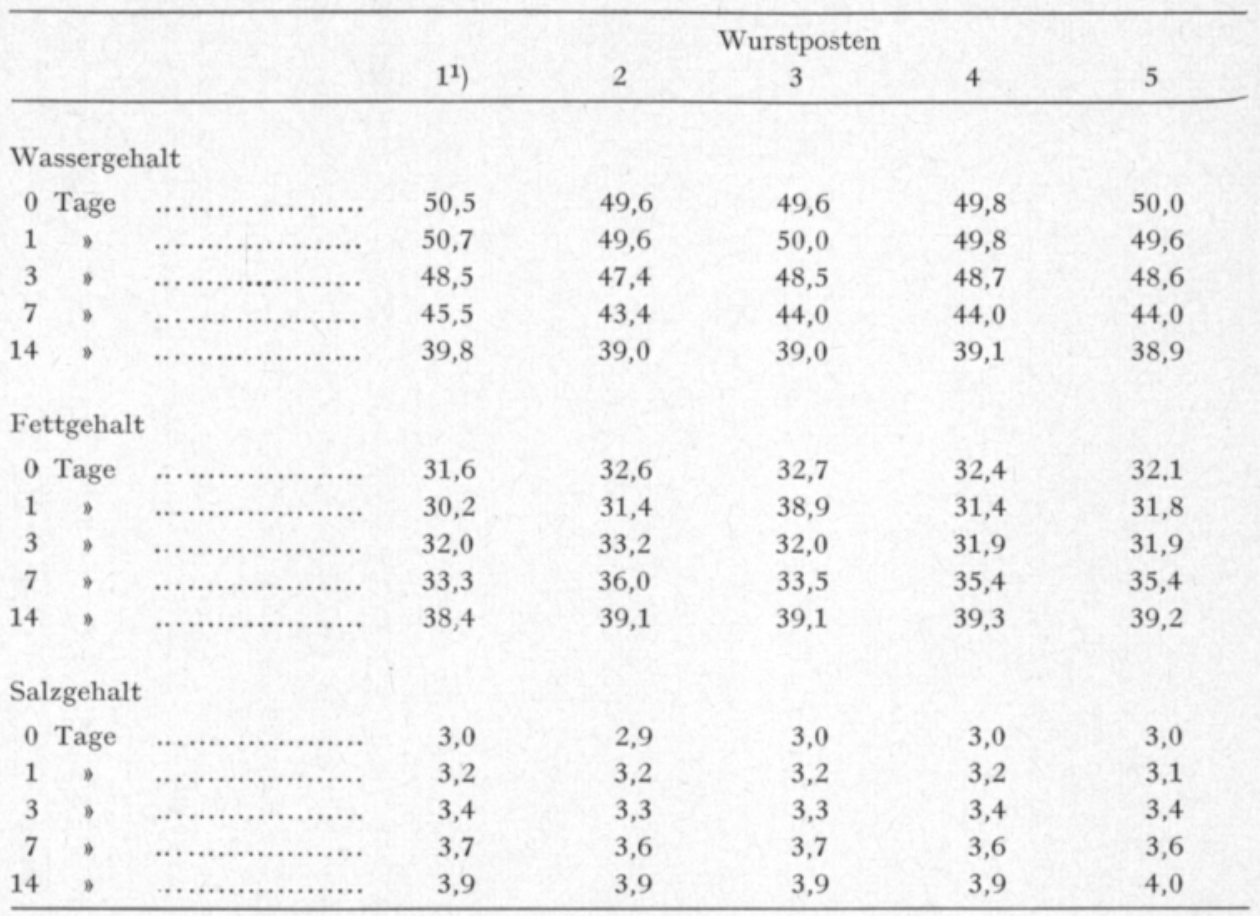

1) Siehe Tabelle 5

Tabelle 15. Nitritgehalte der Würste der vergleichenden Versuchsreihen V1-V6, (ppm).

\begin{tabular}{|c|c|c|c|c|c|c|c|}
\hline \multirow[b]{2}{*}{$\begin{array}{l}\text { Zeit } \\
\text { (Tage) }\end{array}$} & \multicolumn{5}{|c|}{ Wurstposten ${ }^{1}$ ) } & \multirow[b]{2}{*}{ S.d.U. } & \multirow{2}{*}{$\begin{array}{l}\text { k.s.U. } \\
\text { (S.d.U. = } \\
0.05)\end{array}$} \\
\hline & 1 & 2 & 3 & 4 & 5 & & \\
\hline \multirow{2}{*}{$\begin{array}{ll}0 & \overline{\mathrm{x}} \\
& \mathrm{s}\end{array}$} & 78,8 & 127,5 & 1,5 & 1,2 & 37,8 & & \\
\hline & 5,6 & 13,0 & 0,6 & 0,4 & 6,5 & & \\
\hline \multirow[t]{2}{*}{1} & 14,1 & 35,8 & 5,0 & 7,5 & 87 & & \\
\hline & 2,4 & 2,5 & 5,9 & 12,1 & 5,1 & & \\
\hline \multirow[t]{2}{*}{3} & 10,0 & 21,1 & 17,1 & 28,7 & 17,6 & 0,001 & 5,42 \\
\hline & 1,5 & 1,9 & 5,0 & 6,3 & 4,5 & & \\
\hline \multirow[t]{2}{*}{7} & 9,0 & 14,4 & 10,1 & 13,9 & 10,3 & & \\
\hline & 1,7 & 1,0 & 1,5 & 9,0 & 1,1 & & \\
\hline \multirow[t]{2}{*}{14} & 10,2 & 15,2 & 9,8 & 12,9 & 10,7 & 0,001 & 1,74 \\
\hline & 1,5 & 0,6 & 1,1 & 1,8 & 1,5 & & \\
\hline
\end{tabular}

1) Siehe Tabelle 5

signifikant stärkste Nitritkonzentration und unterschied sich somit in signifikanter Weise von der mit entsprechend grossem Nitratzusatz hergestellten Wurst 4. Die mit dem geringeren Nitrit- bzw. Nitratzusatz hergestellten Würste unterschieden sich nicht signifikant voneinander, wohl aber von den 


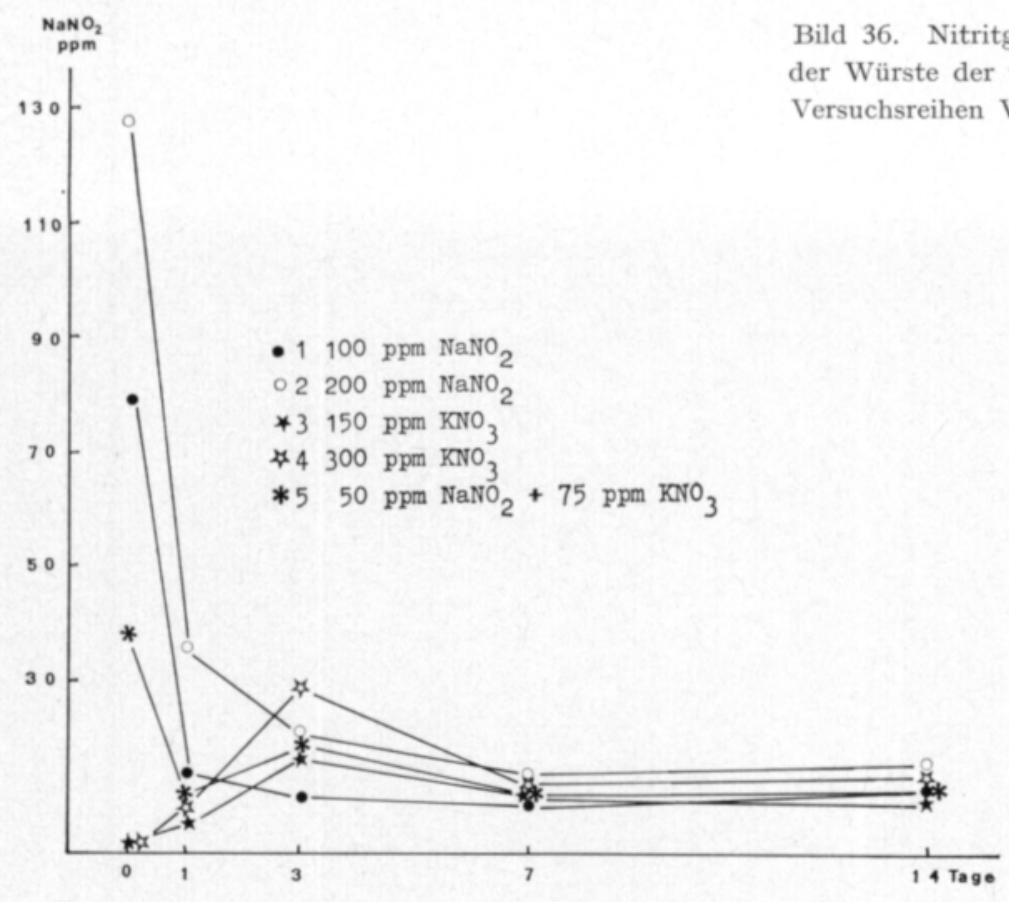

Würsten 2 und 4. Eine praktische Bedeutung kam diesen Differenzen jedoch nicht zu.

Bei der Einschätzung des gesundheitlichen Risikos in seiner Gesamtheit ist auch die Nitratmenge in Rechnung zu stellen. Die Entwicklung des Nitratgehalts im Verlauf des Herstellungsprozesses ist aus Tabelle 16 und Bild 37 ersichtlich. Der Nitratgehalt der GdL-Würste 1 und 2 war anfangs gering, stieg aber in den ersten drei Tagen kräftig an und blieb bis zum Versuchsende auf einem hohen Wert. Die Verringerung der Nitritmenge hatte keinen signi-

Tabelle 16. Nitratgehalte der Würste der vergleichenden Versuchsreihen V1-V6, (ppm).

\begin{tabular}{|c|c|c|c|c|c|c|c|c|}
\hline \multirow{2}{*}{\multicolumn{2}{|c|}{$\begin{array}{l}\text { Zeit } \\
\text { (Tage) }\end{array}$}} & \multicolumn{6}{|c|}{ Wurstposten $^{\mathbf{1}}$ ) } & \multirow{2}{*}{$\begin{array}{l}\text { k.s.U. } \\
\text { (S.d.U. = } \\
0.05)\end{array}$} \\
\hline & & 1 & 2 & 3 & 4 & 5 & S.d.U. & \\
\hline \multirow[t]{2}{*}{0} & $\overline{\mathbf{x}}$ & 16,6 & 46,4 & 137,3 & 255,3 & 78,3 & & \\
\hline & s & 8,6 & 28,0 & 25,6 & 59,8 & 13,8 & & \\
\hline \multirow{2}{*}{\multicolumn{2}{|c|}{1}} & 69,8 & 46,4 & 139,1 & 262,0 & 115,8 & & \\
\hline & & 19,8 & 14,5 & 27,0 & 40,5 & 18,0 & & \\
\hline \multirow{2}{*}{\multicolumn{2}{|c|}{3}} & 92,1 & 71,8 & 40,9 & 111,7 & 61,4 & 0.001 & 25,6 \\
\hline & & 22,1 & 22,6 & 18,0 & 29,4 & 9,4 & & \\
\hline \multirow{2}{*}{\multicolumn{2}{|c|}{7}} & 84,2 & 87,3 & 20,1 & 54,0 & 21,6 & & \\
\hline & & 15,8 & 32,8 & 2,9 & 15,4 & 6,2 & & \\
\hline \multirow{2}{*}{\multicolumn{2}{|c|}{14}} & 99,4 & 99,7 & 20,9 & 28,4 & 21,3 & 0,001 & 16,8 \\
\hline & & 20,6 & 19,4 & 4,2 & 7,1 & 5,5 & & \\
\hline
\end{tabular}

1) Siehe Tabelle 5 


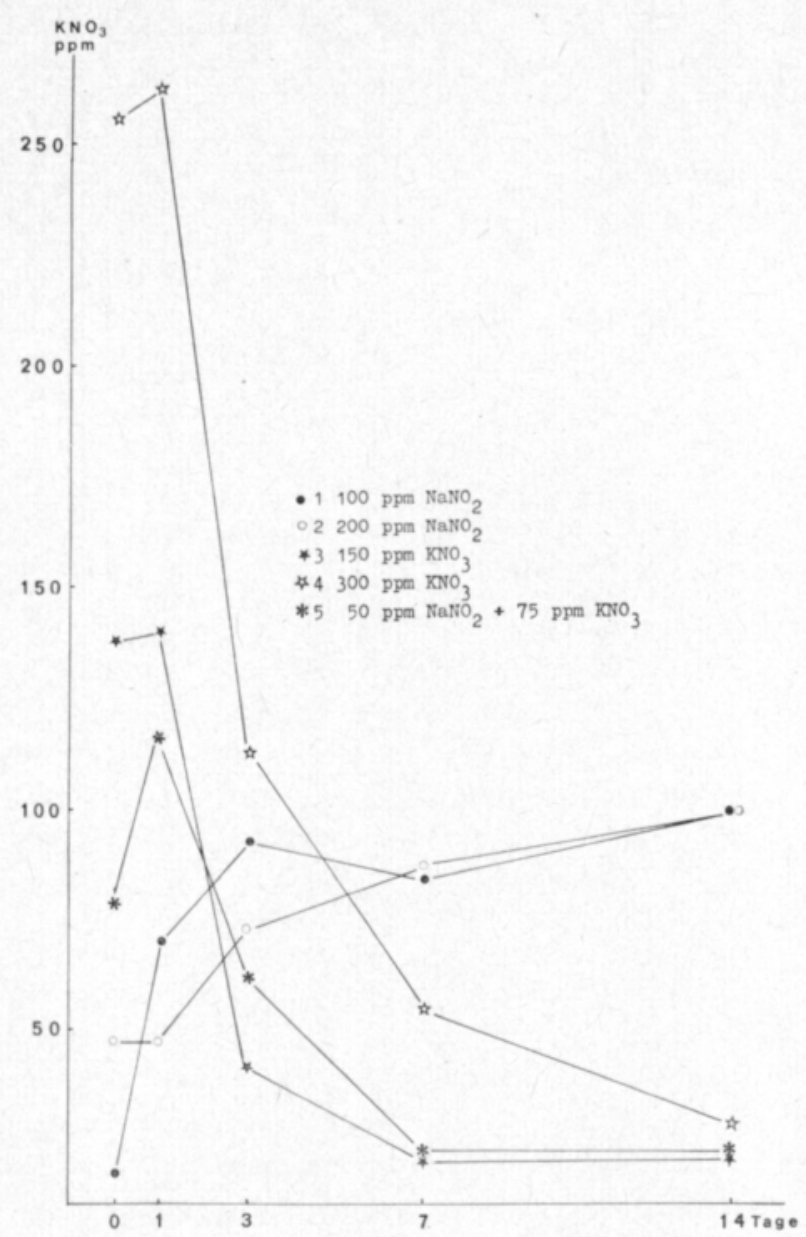

Bild 37. NitratgehaltsMittelwerte der Würste der vergleichenden Versuchsreihen V1-V6.

fikanten Einfluss auf den Restnitratgehalt. Die Starterkultur-Würste hatten anfangs einen hohen Nitratgehalt, der jedoch nach drei Tagen etwa auf das Niveau der GdL-Würste abgesunken war. Bei der mit Nitrit-Nitrat-Zusatz hergestellten Wurst 5 stieg der Nitratgehalt zunächst an, und nach drei Tagen wies diese Wurst mehr Nitrat als die Wurst 3 auf. Nach 14 Tagen hatten dei Starterkultur-Würste etwa ein Viertel des Nitratgehalts der GdL-Würste, Signifikante Unterschiede waren in keiner der beiden Gruppen festzustellen jedoch enthielt die mit dem höheren Nitratzusatz hergestellte Wurst 4 mehr Nitrat als die mit dem geringeren Zusatz hergestellten Würste 3 und $\mathbf{5}$.

Da die Würste einen höheren Nitrat- als Nitritgehalt hatten, war ersterer bestimmend in bezug auf die Unterschiede des Gesamtnitritäquivalentgehalts (Tabelle 17), was auch in den zum Nitratgehalt analogen Ergebnissen zum Ausdruck kommt. In Tabelle 17 ist die in Nitritäquivalente umgerechnete Nitrit-Nitrat-Gesamtmenge in Prozent der zugesetzten Menge angegeben; ausserdem sind die Werte - abweichend von den vorangehenden Tabellen - dem Gewichtsverlust $\mathrm{entsprechend} \mathrm{korrigiert,} \mathrm{so} \mathrm{dass} \mathrm{ersichtlich} \mathrm{ist,} \mathrm{wieviel}$ 
Tabelle 17. Nitrit-Nitrat-Gesamtmengen-Mittelwerte der Würste der vergleichenden Versuchsreihen V1-V6, gerechnet als $\mathrm{NaNO}_{2}-\mathrm{A}$ quivalente und ausgedrückt in Prozent der zugesetzten Menge unter Berücksichtigung des Gewichtsverlustes der Würste.

\begin{tabular}{cccccc}
\hline $\begin{array}{c}\text { Zeit } \\
\text { (Tage) }\end{array}$ & 1 & 2 & 3 & 4 & 5 \\
\hline 0 & 90,2 & 79,7 & 93,1 & 85,7 & 93,9 \\
1 & 58,6 & 33,2 & 95,8 & 88,7 & 85,3 \\
3 & 68,4 & 32,8 & 40,9 & 47,8 & 53,1 \\
7 & 59,9 & 32,5 & 20,4 & 21,7 & 21,6 \\
14 & 63,3 & 33,3 & 18,7 & 12,4 & 19,9 \\
\hline
\end{tabular}

1) Siehe Tabelle 5

des zugesetzten Nitrits bzw. Nitrats an den einzelnen Stichtagen noch vorhanden war.

Wie aus Tabelle 17 hervorgeht, nahm bei den Starterkultur-Würsten die Nitrit-Nitrat-Gesamtmenge während des Herstellungsprozesses ständig ab, während bei den GdL-Würsten der Rückgang bereits nach einem Tag zum Stillstand kam. Der relative Rückgang war um so stärker, je mehr Nitrit bzw. Nitrat zu Beginn zugesetzt worden war.

\subsubsection{Stickoxidmyoglobin-Gehalt}

In der vorliegenden Arbeit wurde die an Hämin gebundene Stickoxidmenge (NO) bestimmt und als Stickoxidmyoglobin (NOMb) angegeben, unabhängig davon ob das Stickoxid eventuell an undenaturiertes Globin (Stickoxidmyoglobin) oder an denaturiertes Globin (Stickoxidmyochromogen) gebunden war (Tabelle 18).

Unter Berücksichtigung des Gewichtsverlustes (Tabelle 26) kann konstatiert werden, dass die NOMb-Menge nach dem dritten Tag nicht mehr wesentlich zunahm. Bei den mit den niedrigeren Nitrit- und/oder Nitratzusätzen hergestellten Würsten stieg der NOMb-Gehalt, wobei in den Starterkultur-Würsten eine stärkere Zunahme als bei den GdL-Würsten zu beobachten war. Bei den Würsten mit dem höheren Nitrit- oder Nitratzusatz ging die NOMb-Menge sogar etwas zurück.

Nach drei Tagen wiesen die Würste keine NOMb-Gehaltsunterschiede auf, aber nach 14 Tagen wurde dann bei der Wurst 1 ein signifikant geringerer NOMb-Gehalt als bei den Würsten 3, 4 und 5 festgestellt.

\subsubsection{Mikrobiologische Ergebnisse}

Die Gesamtbakterienzahlen sind in Tabelle 19 zusammengestellt. Bei den GdL-Würsten stieg die Gesamtbakterienzahl nach dem dritten Tag um etwa 1,5 logarithmische Einheiten an. Nach 14 Tagen wies die Wurst 2 einen etwas geringeren Bakterienbesatz als die Wurst 1 auf. Bei der Gesamtbakterienzahl der Starterkultur-Würste zeigten sich keine wesentlichen Veränderungen; allerdings hatte die Wurst 4 am Ende des Versuchs den nied- 
Tabelle 18. Stickoxidmyoglobin-Menge (NOMb, mg/g). Würste der vergleichenden Versuchsreihen V1-V6.

\begin{tabular}{|c|c|c|c|c|c|c|c|}
\hline \multirow{2}{*}{$\begin{array}{c}\text { Zeit } \\
\text { (Tage) }\end{array}$} & \multicolumn{5}{|c|}{ Wurstposten $^{2}$ ) } & \multirow[t]{2}{*}{ S.d.U. } & \multirow{2}{*}{$\begin{array}{c}\text { k.s.U. } \\
\text { (S.d.U. }= \\
\quad 0.05)\end{array}$} \\
\hline & 1 & 2 & 3 & 4 & 5 & & \\
\hline$\overline{\mathbf{x}}$ & 4.65 & 5.25 & 4.70 & 5.42 & 5.01 & $>0,05$ & - \\
\hline s & 0,76 & 0,94 & 0,99 & 1,67 & 1,18 & & \\
\hline \multirow[t]{2}{*}{14} & 5,63 & 6,05 & 6,32 & 6,25 & 6,47 & 0,05 & 0,62 \\
\hline & 0,48 & 0,45 & 0,50 & 0,63 & 0,71 & & \\
\hline $\begin{array}{l}\text { Zusatz zum } \\
\text { 3-Tage-Wert'1) }\end{array}$ & $5,1 \%$ & $-0,7 \%$ & $15,8 \%$ & $-1,6 \%$ & $11,4 \%$ & & \\
\hline
\end{tabular}

1) Gewichtsverlust berücksichtigt

2) Siehe Tabelle 5

Tabelle 19. Gesamtbakterienzahl $(\log N$, Keime/g) in den vergleichenden Versuchsreihen V1 - V6

\begin{tabular}{|c|c|c|c|c|c|}
\hline $\begin{array}{c}\text { Zeit } \\
\text { (Tage) }\end{array}$ & 1 & 2 & $\begin{array}{c}\text { Wurstposten }{ }^{1} \text { ) } \\
3\end{array}$ & 4 & 5 \\
\hline 0 & 5,68 & 5,69 & 7,31 & 7,35 & 7,34 \\
\hline 1 & 5,91 & 5,12 & 7,37 & 7,31 & 7,29 \\
\hline 3 & 5,75 & 5,59 & 7,54 & 7,52 & 7,52 \\
\hline 7 & 7,19 & 6,97 & 7,49 & 7,36 & 7,67 \\
\hline 14 & 7,26 & 7,02 & 7,28 & 7,03 & 7,45 \\
\hline
\end{tabular}

1) Siehe Tabelle 5

rigsten Bakteriengehalt. Die grösste Nitrit- bzw. Nitratzugabe senkte somit die Gesamtbakterienzahl um durchschnittlich 0,3 logarithmische Einheiten. Allerdings ist hierzu festzustellen, dass - nach Tabelle 20 - die Wurst 4 nach 14 Tagen einen Laktobazillengehalt von $\log \mathrm{N}=7,35$ aufwies, wohingegen nach Tabelle 19 die Gesamtbakterienzahl $\log \mathrm{N}=7,03$ betrug, so dass nicht alle der in SL-Nährboden gediehenen Bakterien auch in PC-Nährboden gediehen waren.

Tabelle 20. Laktobazillenzahl $(\log \mathrm{N}, \mathrm{Keime} / \mathrm{g})$ in den vergleichenden Versuchsreihen V1-V6.

\begin{tabular}{cccccc}
\hline $\begin{array}{c}\text { Zeit } \\
\text { (Tage) }\end{array}$ & 1 & 2 & \multicolumn{5}{c}{ Wurstposten $^{1}$ ) } & \\
\hline & & & 3 & 4 & \\
& 2,46 & 2,06 & 7,31 & 7,28 & 7,25 \\
1 & 2,90 & 2,62 & 7,25 & 7,22 & 7,20 \\
3 & 5,72 & 5,36 & 7,58 & 7,61 & 7,43 \\
7 & 6,80 & 6,54 & 7,49 & 7,20 & 7,39 \\
14 & 7,01 & 6,85 & 7,27 & 7,35 & 7,61 \\
\hline
\end{tabular}

1) Siehe Tabelle 5 
Bei den GdL-Würsten fand zwischen dem zweiten und siebten Tag ein steiler Anstieg der Laktobazillenzahl statt. Der Unterschied zwischen den Würsten 1 und 2 war jedoch gering. Bei den Starterkultur-Würsten blieb die Laktobazillenmenge während der gesamten Versuchsdauer etwa auf gleichem Niveau, und auch der Quantität des Nitratzusatzes schien in diesem Falle keine Bedeutung zuzukommen.

Tabelle 21. Mikrokokkenzahl ( $\log \mathrm{N}, \mathrm{Keime} / \mathrm{g})$ in den vergleichenden Versuchsreihen V1-V6.

\begin{tabular}{cccccc}
\hline $\begin{array}{c}\text { Zeit } \\
\text { (Tage) }\end{array}$ & 1 & 2 & \multicolumn{5}{c}{ Wurstposten $^{1}$ ) } & 5 \\
\hline 0 & 4,27 & 3,83 & 7,26 & 7,14 & 7,07 \\
1 & 4,12 & 3,90 & 7,23 & 7,21 & 7,12 \\
3 & 4,22 & 3,99 & 6,94 & 7,00 & 7,03 \\
7 & 4,13 & 3,91 & 6,67 & 6,79 & 6,69 \\
14 & 4,12 & 3,62 & 6,74 & 6,73 & 6,81 \\
\hline
\end{tabular}

1) Siehe Tabelle 5

Die Mikrokokkenzahl (Tabelle 21) der GdL-Würste nahm während der gesamten Versuchsdauer etwas ab. Die stärker nitrithaltige Wurst 2 enthielt von Anfang an weniger Mikrokokken, aber zwischen den Würsten 1 und 2 bestand kein wesentlicher Unterschied, es sei denn, dass eine unmittelbare Wirkung des Nitrits auf einen Teil der Mikrokokken gleich am Herstellungstag vorlag. Bei den Starterkultur-Würsten wurde auch ein leichter Rückgang der Mikrokokkenzahl beobachtet, Unterschiede zwischen den Würsten waren jedoch nicht festzustellen.

Die Anzahl der fäkalen Streptokokken, Kolibakterien und Hefezellen lag bei den Würsten der vergleichenden Versuchsreihen so nahe bei der Feststellbarkeitsgrenze (100 Keime/g), dass eine Auswertung des Ergebnismaterials nicht möglich war. Infolge der zahlreichen leeren Plattén konnten keine Mittelwerte berechnet werden. Ganz allgemein sei festgestellt, dass die o.g. Mikroben in den Versuchsreihen keinerlei Problem darstellten, denn sie vermehrten sich während der Dauer der Versuche kaum.

In einigen wenigen Fällen enthielten die Starterkultur-Würste die oben angeführten Mikroben anfangs in etwas grösseren Mengen als die GdL-Würste, jedoch waren diese nach sieben und 14 Tagen nahezu ausnahmslos auf einen Wert unter $100 \mathrm{Keime} / \mathrm{g}$ gesunken.

\subsection{5 pH-Wert, $a_{\mathrm{w}}$-Wert, Konsistenz und Gewichtsverlust}

In Tabelle 22 und Bild 38 ist die pH-Entwicklung bei den Würsten der vergleichenden Versuchsreihen dargestellt. Bei den GdL-Würsten nahm der $\mathrm{pH}$ bereits während des Kutterns ab und lag somit schon am Herstellungstag niedriger als bei den Starterkultur-Würsten. Nach einem Tag hatten die GdL-Würste schon nahezu ihren End-pH erreicht, während der $\mathrm{pH}-$ Wert der Starterkultur-Würste gegenüber dem Anfangswert kaum Änderungen erfahren 
Tabelle 22. pH-Entwicklung bei den Würsten der vergleichenden Versuchsreihen V1-V6.

\begin{tabular}{|c|c|c|c|c|c|c|c|c|}
\hline \multirow{2}{*}{\multicolumn{2}{|c|}{$\begin{array}{c}\text { Zeit } \\
\text { (Tage) }\end{array}$}} & \multicolumn{5}{|c|}{ Wurstposten $^{\mathbf{1}}$ ) } & \multirow[t]{2}{*}{ S.d.U. } & \multirow{2}{*}{$\begin{array}{c}\text { k.s.U. } \\
\text { (S.d.U. }= \\
0.05)\end{array}$} \\
\hline & & 1 & 2 & 3 & 4 & 5 & & \\
\hline \multirow[t]{2}{*}{0} & $\overline{\mathbf{x}}$ & 5,36 & 5,40 & 5,61 & 5,61 & 5,61 & & \\
\hline & s & 0,17 & 0,15 & 0,08 & 0,07 & 0,09 & & \\
\hline \multirow[t]{2}{*}{1} & & 5,07 & 5,06 & 5,47 & 5,50 & 5,49 & & \\
\hline & & 0,04 & 0,04 & 0,06 & 0,07 & 0,05 & & \\
\hline \multirow[t]{2}{*}{3} & & 5,07 & 5,10 & 5,12 & 5,10 & 5,20 & $>0,05$ & - \\
\hline & & 0,05 & 0,04 & 0,12 & 0,08 & 0,13 & & \\
\hline \multirow[t]{2}{*}{7} & & 5,00 & 5,04 & 4,83 & 4,83 & 4,87 & & \\
\hline & & 0,09 & 0,09 & 0,10 & 0,08 & 0,06 & & \\
\hline \multirow[t]{2}{*}{14} & & 4,94 & 5,01 & 4,72 & 4,76 & 4,78 & 0,01 & 0,20 \\
\hline & & 0,14 & 0,10 & 0,12 & 0,07 & 0,09 & & \\
\hline
\end{tabular}

1) Siehe Tabelle 5

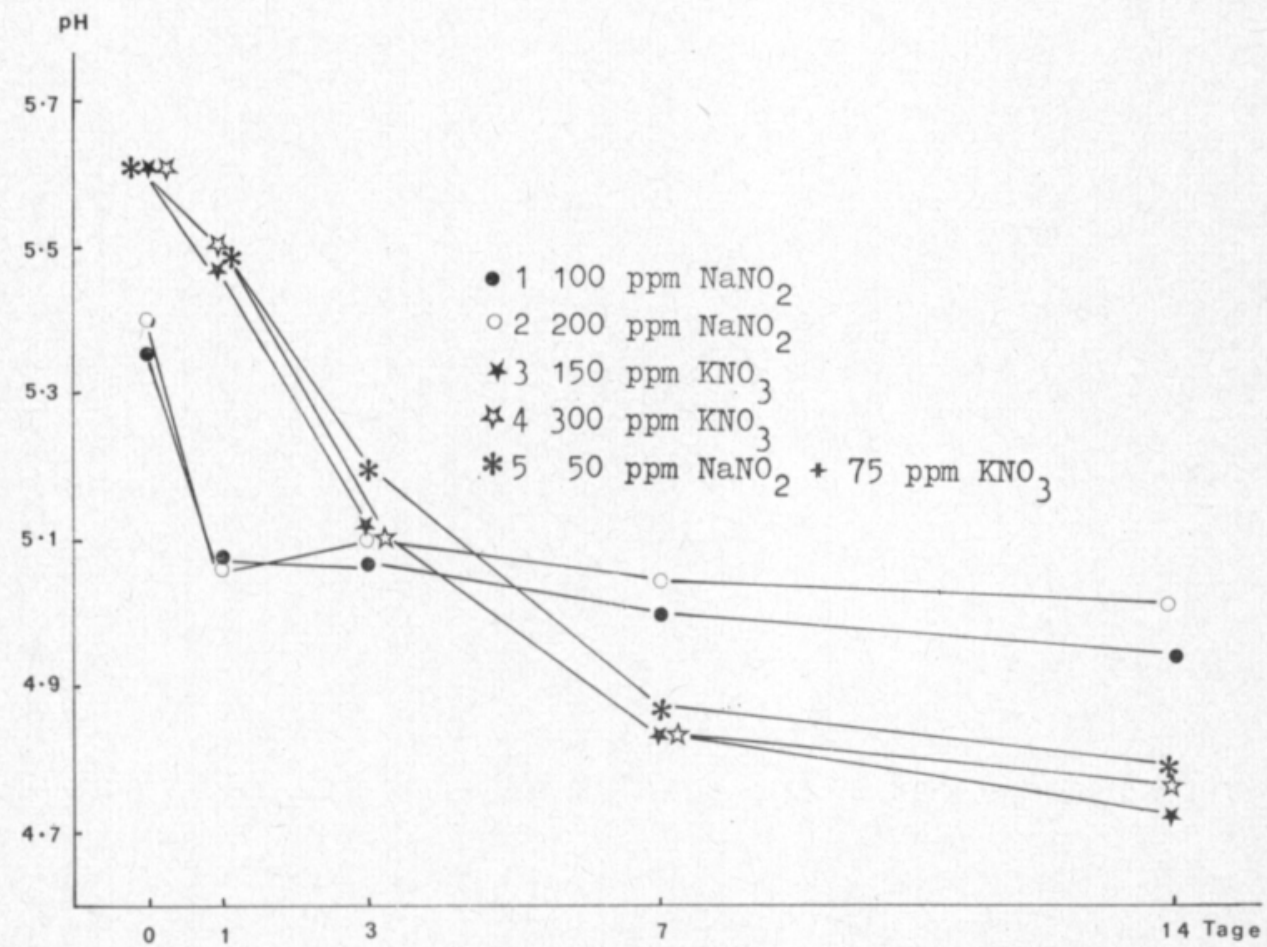

Bild 38. Mittelwerte der pH-Werte der Würste aus den vergleichenden Versuchsreihen V1 - V6.

hatte. Nach drei Tagen bewegte sich der pH der Starterkultur-Würste bereits so nahe beim $\mathrm{pH}-$ Wert der GdL-Würste, dass zwischen ersteren und letzteren keine statistisch signifikanten Unterschiede mehr bestanden.

Nach 14 Tagen betrug der $\mathrm{pH}$ der Starterkultur-Würste 4,7-4,8, der der GdL-Würste etwa 5,0. Die mit dem höheren Nitrit- bzw. Nitratzusatz her- 
gestellten Würste zeigten einen etwas höheren $\mathrm{pH}$ als die entsprechenden Würste mit dem niedrigeren Nitrit- bzw. Nitratzusatz. Bei den StarterkulturWürsten wies allerdings die Wurst 5 den höchsten $\mathrm{pH}$-Wert auf. Die Wurst 2 hatte einen signifikant höheren $\mathrm{pH}$ als die Starterkultur-Würste, die Wurst 1 einen signifikant höheren $\mathrm{pH}$ als die Wurst 3. Hierbei ist jedoch zu berücksichtigen, dass die Änderungen des pH-Werts wegen dessen logarithmischen Charakters in keinem direkten Verhältnis zur Säurekonzentration stehen. Auch das Pufferungsvermögen des Fleisches stellt in diesem Bereich eine beachtenswerte Variable dar. Dazu kommt, dass der Einfluss der Wasserstoffionenkonzentration auf die technologischen, mikrobiologischen und organoleptischen Eigenschaften der Rohwurst in diesem $\mathrm{pH}$-Bereich wohl kaum ein linearer ist. Rein statistische Betrachtungen des $\mathrm{pH}$-Werts bieten deshalb nur sehr ungenügende Anhaltspunkte bei der Beurteilung dessen Auswirkungen. Hinsichtlich der $a_{W}$-Werte wurden zwischen den Würsten keine statistisch signifikanten Unterschiede festgestellt (Tabelle 23).

Tabelle 23. $a_{w}$-Werte der Würste der vergleichenden Versuchsreihen V1-V6.

\begin{tabular}{|c|c|c|c|c|c|c|c|c|}
\hline & $\begin{array}{l}\text { Zeit } \\
\text { (Tage) }\end{array}$ & 1 & 2 & $\begin{array}{l}\text { urstpost } \\
3\end{array}$ & 4 & 5 & S.d.U. & $\begin{array}{l}\text { k.s.U. } \\
\text { (S.d.U. = } \\
0.05)\end{array}$ \\
\hline \multirow[t]{2}{*}{0} & $\bar{x}$ & 0,952 & 0,951 & 0,954 & 0,955 & 0,953 & & \\
\hline & s & 2 & 1 & 2 & 2 & 2 & & \\
\hline \multirow[t]{2}{*}{1} & & 0,949 & 0,949 & 0,950 & 0,951 & 0,951 & & \\
\hline & & 4 & 3 & 3 & 3 & 3 & & \\
\hline \multirow[t]{2}{*}{3} & & 0,944 & 0,943 & 0,944 & 0,945 & 0,944 & $>0,05$ & - \\
\hline & & 2 & 3 & 2 & 2 & 3 & & \\
\hline \multirow[t]{2}{*}{7} & & 0,931 & 0,932 & 0,933 & 0,933 & 0,934 & & \\
\hline & & 3 & 2 & 2 & 1 & 1 & & \\
\hline \multirow[t]{2}{*}{14} & & 0,916 & 0,914 & 0,913 & 0,917 & 0,914 & $>0,05$ & - \\
\hline & & 4 & 4 & 5 & 3 & 4 & & \\
\hline
\end{tabular}

1) Siehe Tabelle 5

Die Konsistenzwerte (Tabelle 24) folgten konsequent der pH-Wert-Entwicklung. Die zwischen $\mathrm{pH}$ und Konsistenzwert festgestellten Korrelationskoeffizienten gehen aus Tabelle 25 hervor. Am dritten Tag hatten die GdL-Würste eine festere Konsistenz als die Starterkultur-Würste (Tabelle 24). Die Würste 1 und 2 waren signifikant fester als die Wurst 5. Am 14. Tag hatten sämtliche Starterkultur-Würste eine signifikant bessere Konsistenz als die GdL-Würste. In beiden Gruppen war die Festigkeitsrangfolge umgekehrt proportional zur pH-Reihenfolge.

Bezüglich der Festigkeit wurden am dritten Tag, organoleptisch beurteilt, keine signifikanten Unterschiede festgestellt (Tabelle 24). Nach sieben Tagen unterschieden sich die Starterkultur-Würste signifikant von den GdL-Wüısten, jedoch kam dem Nitrit- bzw. Nitratzusatz in dieser Hinsicht keine Bedeutung zu. Nach vierzehn Tagen wurde die Konsistenz der Starterkultur-Würste 
Tabelle 24. Konsistenzwerte (kg) und organoleptisch beurteilte Konsistenzpunktzahlen (maxim. 2) der Würste der vergleichenden Versuchsreihen V1-V6.

\begin{tabular}{|c|c|c|c|c|c|c|c|}
\hline \multirow{2}{*}{$\begin{array}{c}\text { Zeit } \\
\text { (Tage) }\end{array}$} & \multirow[b]{2}{*}{1} & \multicolumn{3}{|c|}{ Wurstposten $^{1}$ ) } & \multirow[b]{2}{*}{5} & \multirow{2}{*}{ S.d.U. } & \multirow{2}{*}{$\begin{array}{r}\text { k.s.U. } \\
\text { (S.d.U. }= \\
0.05)\end{array}$} \\
\hline & & 2 & 3 & 4 & & & \\
\hline \multicolumn{8}{|l|}{ Gemessen } \\
\hline \multirow{2}{*}{1} & 2,3 & 2,3 & 1,4 & 1,3 & 1,4 & & \\
\hline & 0,2 & 0,2 & 0,2 & 0,2 & 0,2 & & \\
\hline \multirow[t]{2}{*}{3} & 2,6 & 2,4 & 2,2 & 2,2 & 2,0 & 0,01 & 0,4 \\
\hline & 0,3 & 0,3 & 0,2 & 0,3 & 0,2 & & \\
\hline \multirow[t]{2}{*}{7} & 3,1 & 2,9 & 4,0 & 4,0 & 3,8 & & \\
\hline & 0,4 & 0,5 & 0,9 & 0,6 & 0,5 & & \\
\hline \multirow[t]{2}{*}{14} & 4,6 & 4,8 & 6,8 & 6,5 & 6,3 & 0,001 & 1,2 \\
\hline & 0,6 & 0,6 & 1,1 & 0,9 & 0,9 & & \\
\hline \multirow{2}{*}{\multicolumn{8}{|c|}{$\begin{array}{l}\text { Organoleptisch } \\
\text { beurteilt }\end{array}$}} \\
\hline & & & & & & & \\
\hline \multirow[t]{2}{*}{3} & 0,9 & 0,8 & 0,9 & 1,0 & 0,9 & $>0,05$ & - \\
\hline & 0,2 & 0,2 & 0,3 & 0,5 & 0,4 & & \\
\hline \multirow[t]{2}{*}{7} & 0,8 & 0,8 & 1,4 & 1,6 & 1,3 & 0,001 & 0,45 \\
\hline & 0,2 & 0,2 & 0,5 & 0,2 & 0,6 & & \\
\hline \multirow[t]{2}{*}{14} & 1,1 & 1,4 & 1,9 & 1,8 & 1,8 & 0,001 & 0,43 \\
\hline & 0,3 & 0,3 & 0,1 & 0,3 & 0,2 & & \\
\hline
\end{tabular}

1) Siehe Tabelle 5

punktmässig höher bewertet als die der GdL-Würste. Die Konsistenz-Punktzahl der Wurst 1 lag signifikant niedriger als die entsprechenden Werte der Starterkultur-Würste. Der Nitrit- bzw. Nitratzusatz war ohne Belang. Allerdings erreichte die Wurst 2 eine etwas höhere Punktzahl als die Wurst 1, obwohl auf Grund der $\mathrm{pH}$-Werte eigentlich das umgekehrte Ergebnis zu erwarten gewesen wäre (Tabelle 21).

Nach Tabelle 25 bestand am dritten Tag zwischen dem Konsistenzwert und der organoleptischen Beurteilung keine Korrelation, wohingegen am siebten Tag bereits ein sehr hoher Korrelationskoeffizient, $r=+0,963$, vorlag.

Tabelle 25. Korrelationskoeffizienten (r) zwischen pH-Wert und Konsistenzwert sowie zwischen gemessener und organoleptisch bestimmter Konsistenzpunktzahl.

\begin{tabular}{lrr}
\hline & $\begin{array}{c}\text { Zeit } \\
\text { (Tage) }\end{array}$ & \multicolumn{1}{c}{ r } \\
\hline pH-Wert - Konsistenzwert (gemessen) & 1 & $-0,999$ \\
& 3 & $-0,906$ \\
Konsistenzwert (gemessen) - & 7 & $-1,000$ \\
Konsistenz (organoleptisch beurteilt) & 14 & $-0,964$ \\
& 3 & $-0,312$ \\
& 7 & $+0,963$ \\
\hline
\end{tabular}


Beim Gewichtsverlust (Tabelle 26) wurden zwischen den einzelnen Würsten keine statistisch signifikanten Unterschiede festgestellt; allerdings wiesen die Starterkultur-Würste einen geringfügig höheren Gewichtsverlust als die GdLWürste auf. Möglicherweise war die Versuchsdauer (14 Tage) für das Entstehen wesentlicher Differenzen zu kurz.

Tabelle 26. Gewichtsverlust bei den Würsten der Versuchsreihen V1-V6 (\%).

\begin{tabular}{rrrrrrrr}
\hline $\begin{array}{c}\text { Zeit } \\
\text { (Tage) }\end{array}$ & 1 & 2 & $\begin{array}{c}\text { Wurstposten }{ }^{1} \text { ) } \\
3\end{array}$ & 4 & 5 & S.d.U. & $\begin{array}{r}\text { k.s.U. } \\
\text { (S.d.U. }= \\
0.05)\end{array}$ \\
\hline 1 & 1,7 & 1,9 & 2,2 & 2,5 & 1,7 & & \\
3 & 6,3 & 6,4 & 6,8 & 6,7 & 6,7 & $<0,05$ & - \\
7 & 12,2 & 12,4 & 12,3 & 12,8 & 12,9 & & - \\
14 & 19,5 & 20,2 & 20,7 & 21,3 & 20,4 & $<0,05$ & - \\
\hline
\end{tabular}

1) Siehe Tabelle 5

\subsection{Farbe}

Sämtliche Würste wiesen bereits im Alter von einem Tag rote Schnittflächenfarbe auf. Die GdL-Würste hatten einen dünnen grauen Ring; die Wurst 1 einen etwas dickeren als die Wurst 2 (Foto 1). Am dritten Tag war bei den Starterkultur-Würsten eine Intensivierung vor allem der Oberflächenfärbung eingetreten, wohingegen die GdL-Würste an der Oberfläche noch leicht grau waren (Foto 2). Am siebten Tag hatten sämtliche Würste eine rote Schnittflächenfarbe, an der Oberfläche waren die GdL-Würste jedoch nach wie vor grau; das gleiche Bild bot sich auch am 14. Tag (Foto 3 und 4). Am 22. Tag zeigte das Schnittbild der GdL-Würste, verglichen an dem der Starterkultur-Würste, einen Stich ins Bläuliche. Die GdL-Würste hatten eine etwas schlechtere Farbbeständigkeit als die Starterkultur-Würste; ihre Farbe schlug ziemlich schnell in Graubraun um (Foto 5).

Organoleptisch beurteilt war die Schnittflächenfarbe der Wurst 1 am dritten Tag signifikant schwächer als die der Würste 2 und 4 ; die Wurst 5 wiederum hatte eine schlechtere Schnittflächenfarbe als die Wurst 4 (Tabelle 27). Die Oberflächenfarbe der Würste 1 und 2 war signifikant schlechter als die der Würste $3-\mathbf{5}$, ausserdem hatte die Wurst 1 gegenüber der Wurst 2 eine signifikant schwächere Oberflächenfarbe. Am siebten Tag war die Situation bezüglich der Oberfläche die gleiche mit dem Unterschied, dass die Wurst 1 und die Wurst 2 nicht mehr signifikant verschieden waren. Am siebten Tag zeigten sämtliche Starterkultur-Würste eine signifikant bessere Schnittflächenfarbe als die GdL-Würste. Am 14. Tag hatte die Wurst 1 eine signifikant schlechtere Oberflächenfarbe als die übrigen Würste; die Wurst 2 hatte eine schwächere Farbe als die Wurst 4. Bei der Schnittflächenfarbe wurden keine statistisch signifikanten Unterschiede festgestellt.

Bei der Beurteilung der Schnittflächenfarbe der 22 Tage alten Würste durch Rangordnungsprüfung wurde die frische Schnittfläche der Wurst 1 farb- 
Tabelle 27. Oberflächenfarbe (maxim. 6), Schnittflächenfarbe (maxim. 6) und Punkte der organoleptischen Beurteilung der Würste der vergleichenden Versuchsreihen V1-V6.

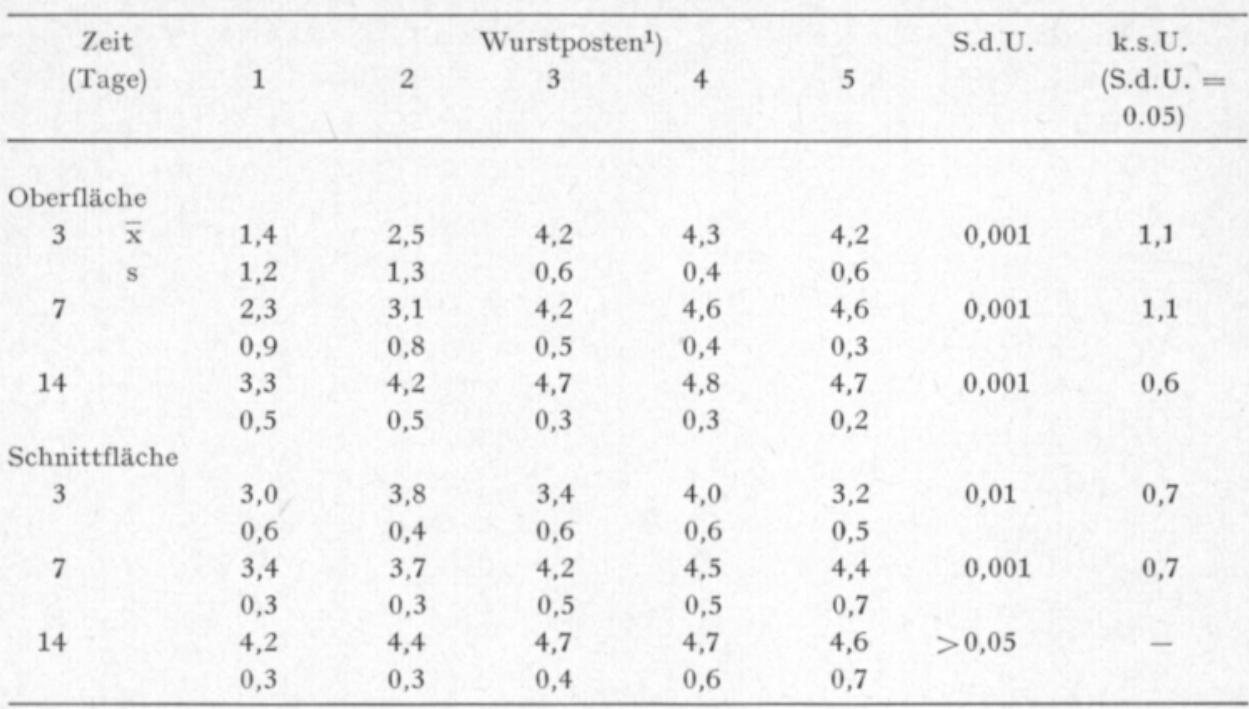

1) Siehe Tabelle 5

mässig für signifikant schwächer, die der Wurst 5 für signifikant besser als die Schnittflächen der anderen Würste befunden. Die Farbe der drei Stunden alten Schnittfläche war bei den Starterkultur-Würsten stark signifikant besser als bei den GdL-Würsten. Hingegen hatten die Nitrit- und Nitratkonzentrationen in dieser Beziehung keinen statistisch signifikanten Einfluss, wenn auch die Rangordnungssummen der mit dem höheren Zusatz hergestellten Würste höher lagen als die der mit dem entsprechenden niedrigeren Zusatz hergestellten Würste (Tabelle 28). Vergleiche zwischen den frischen und den drei Stunden alten Schnittflächen ergaben, dass die Starterkultur-Würste gegenüber den GdL-Würsten eine stark signifikant bessere Farbbeständigkeit aufwiesen.

Tabelle 28. Schnittflächen-Farbbeständigkeit der Würste der vergleichenden Versuchsreihe V6 (Rangordnungssumme $\left.{ }^{1}\right)$ ).

\begin{tabular}{|c|c|c|c|c|c|}
\hline \multirow{2}{*}{ Zeit } & \multicolumn{5}{|c|}{ Wurstposten ${ }^{2}$ ) } \\
\hline & 1 & 2 & 3 & 4 & 5 \\
\hline Frische Schnittfläche ........................... & $69 *$ & 55 & 58 & 50 & $37 *$ \\
\hline 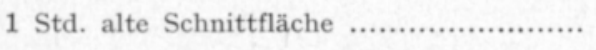 & $\overline{73}^{* *}$ & 66 & 47 & 41 & 46 \\
\hline 3 Stdn. alte Schnittfläche...$\ldots \ldots \ldots \ldots \ldots \ldots \ldots \ldots \ldots \ldots$ & $\overline{71}^{* *}$ & $80^{* *}$ & $39 *$ & $35^{* *}$ & $39 *$ \\
\hline Farbbeständigkeit frisch -3 Stdn. ........... & $\overline{82} * *$ & $\overline{75 * *}$ & $38 * *$ & $35^{* *}$ & $37 * *$ \\
\hline
\end{tabular}

1) Beurteiler $\mathrm{N}=18$ Personen

* Unterschied signifikant, S.d.U. $=0.05 ; * *$ S.d.U. $=0.01 ; \quad$ signifikant schlechter

$\left.{ }^{2}\right)$ Siehe Tabelle 5 


\subsubsection{Geruchs-, Geschmacks- und Gesamtpunkte}

Bezüglich des Geruchs der Würste wurden an keinem der Stichtage signifikante Unterschiede festgestellt. Nach 14 Tagen hatte die Wurst 5 den besten Geschmack, gefolgt von den Würsten 3 und 4 (Tabelle 29). Geschmacklich am schwächsten war die Wurst 1 , die sich in dieser Beziehung signifikant von den Würsten 4 und 5 unterschied. Die mit dem höheren Nitrit- bzw. Nitratzusatz hergestellten Würste erhielten bei der Beurteilung höhere Geschmackspunkte; der Unterschied war jedoch gering und statistisch nicht signifikant. Allerdings erzielte die unter Zusatz einer Nitrit-Nitrat-Mischung hergestellte Wurst 5 die höchste Bewertung, obwohl ihr Nitrit-Nitrat-Zusatz dem niedrigeren Zusatzmittelniveau entsprach (Würste 1 und 3).

Sämtliche Starterkultur-Würste erzielten signifikant höhere Gesamtpunkte als die Wurst 1 , die Würste 3 und 4 wiederum signifikant bessere Gesamtpunkte als die Wurst 2. Bei den GdL-Würsten bewirkte eine Verdopplung des Nitritzusatzes eine Erhöhung der Gesamtpunkte, bei den Starterkultur-Würsten hingegen nur unbedeutende Veränderungen.

Tabelle 29. Bei der organoleptischen Beurteilung für die Würste der vergleichenden Versuchsreihen V1-V6 erteilte Geruchs- (maxim. 2), Geschmacks- (maxim. 4) und Gesamtpunkte (maxim. 20).

\begin{tabular}{|c|c|c|c|c|c|c|c|}
\hline & & & urstp & & & & \\
\hline $\begin{array}{l}\text { Zeit } \\
\text { (Tage) }\end{array}$ & 1 & 2 & 3 & 4 & 5 & S.d.U. & $\begin{array}{l}\text { k.s.U. } \\
\text { (S.d.U. }=0.05)\end{array}$ \\
\hline Geruch & & & & & & & \\
\hline $3 \bar{x}$ & 1,0 & 1,0 & 0,9 & 1,1 & 1,0 & & \\
\hline s & 0,3 & 0,2 & 0,5 & 0,4 & 0,4 & $>0,05$ & - \\
\hline 7 & 1,0 & 1,1 & 1,4 & 1,4 & 1,4 & & \\
\hline & 0,3 & 0,3 & 0,4 & 0,4 & 0,3 & $>0,05$ & - \\
\hline 14 & 1,5 & 1,5 & 1,6 & 1,6 & 1,7 & & \\
\hline & 0,1 & 0,1 & 0,2 & 0,2 & 0,2 & $>0,05$ & - \\
\hline Geschmack & & & & & & & \\
\hline 14 & 2,2 & 2,4 & 2,7 & 2,9 & 3,0 & $>0,05$ & - \\
\hline & 0,5 & 0,2 & 0,4 & 0,6 & 0,3 & & \\
\hline Gesamtpunl & & & & & & & \\
\hline 14 & 10,8 & 12,3 & 13,9 & 14,1 & 14,1 & 0,001 & 1,75 \\
\hline & 1,1 & 0,6 & 0,9 & 0,3 & 1,4 & & \\
\hline
\end{tabular}

1) Siehe Tabelle 5

\subsubsection{Die zwischen Nitrit- und Nitratmenge sowie verschiedenen Eigenschaften der Rohwurst festgestellten Korrelationskoeffizienten}

Der Einfluss der zugesetzten Nitrit- und Nitratmenge bzw. deren Restmenge auf die Eigenschaften der Rohwurst wurde durch Berechnung der betreffenden Korrelationskoeffizienten zu klären versucht (Tabelle 30). Der Nitratgehalt wurde hierzu zunächst in Nitritgehalt umgerechnet. 
Nach Tabelle 30 bestand am dritten und am 14. Tag zwischen der zugesetzten Nitrit-/Nitratmenge und der Restnitritmenge eine starke Korrelation. Zwischen der zugesetzten Nitrit- bzw. Nitratmenge und der NOMb-Menge wurde am dritten Tag eine starke, am 14. Tag jedoch nur eine schwache Korrelation festgestellt. Das gleiche galt auch für die Ergebnisse der organoleptischen Beurteilung sowohl bezüglich des zugesetzten Nitrits als auch des Restnitrits. Diese Ergebnisse zeigten, dass die Nitritkonzentration am ersten Tag nach der Herstellung von Wichtigkeit im Hinblick auf Geschwindigkeit und Quantität der Umrötung ist, dass ihr aber später, nachdem offensichtlich eine Umwandlung des Farbstoffs in die Chromogenform erfolgt ist, keine so grosse Bedeutung mehr zukommt.

Zwischen der zugesetzten Nitritmenge sowie dem Geschmack und der Gesamtpunktzahl bestand keine Korrelation. Die Folgerung daraus ist, dass ein erhöhter Nitrit- bzw. Nitratzusatz zwar zu Beginn des Herstellungsprozesses eine Verbesserung der Rohwursteigenschaften bewirkt, beim fertigen Produkt jedoch keine diesbezüglichen Vorteile mehr festzustellen sind.

Tabelle 30. In den vergleichenden Versuchsreihen V1-V6 zwischen der zugesetzten Nitritund Nitratmenge, der Restmenge und verschiedenen Rohwursteigenschaften festgestellte Korrelationskoeffizienten.

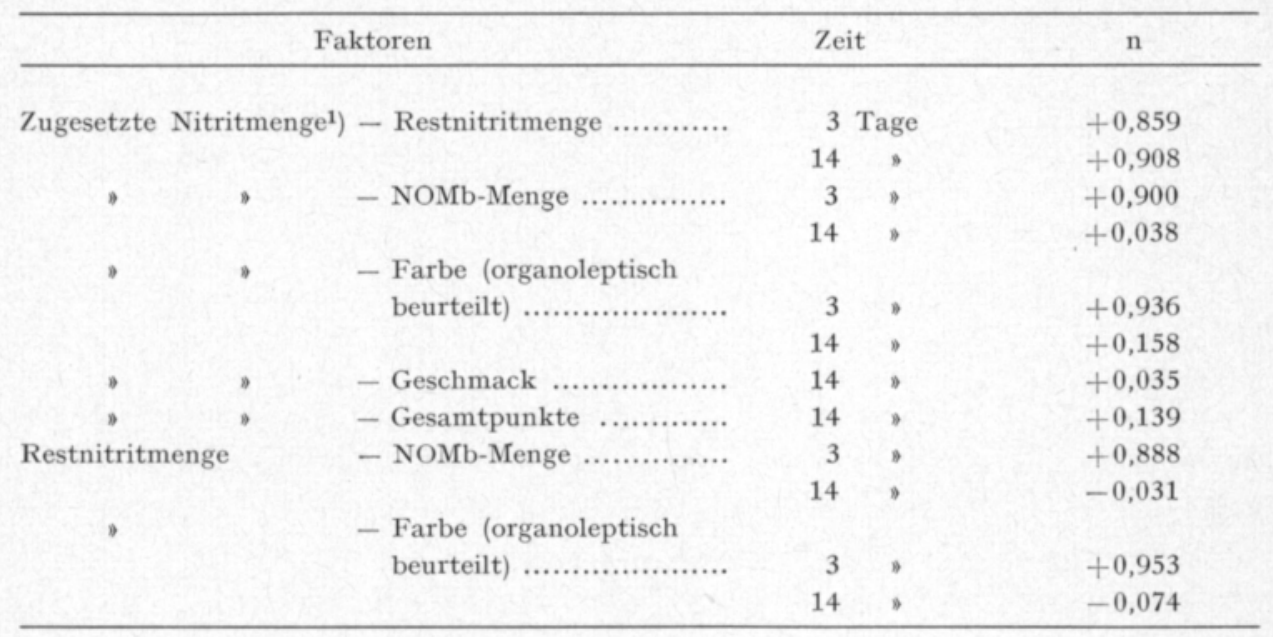

1) einschl. in $\mathrm{NaNO}_{2}$ umgerechneten Nitrats

\section{6 Einfluss von Nitrit und Nitrat auf das Wachstum von Salmonellen}

Die Salmonellen-Versuchsreihe wurde zweifach ausgeführt. Beim ersten Mal betrug der Salmonellenzusatz $0,3-5,0 \cdot 10^{3} \mathrm{Keime} / \mathrm{g}$, beim zweiten Mal $5,4-7,5 \cdot 10^{4} \mathrm{Keime} / \mathrm{g}$. Allerdings ist die Möglichkeit nicht auszuschliessen, dass beim ersten Mal das GdL bereits vor der ersten Bestimmung eine Senkung der Salmonellenzahl bewirkte, denn die GdL-Würste hatten unmittelbar nach der Herstellung nur etwa 1/10 des Salmonellengehalts der Starterkultur-Würste.

Beim zweiten Mal wurde allerdings keine solche Differenz mehr festgestellt (Tabelle 31). In Bild 39 sind die Ergebnisse, die auf der Platte unter der Fest- 
Tabelle 31. Salmonella senftenberg-Anzahl (Keime/g) und pH-Wert der Rohwurst bei Zugabe verschieden grosser Nitrit- und Nitratmengen. Versuchsreihen S1-S2.

\begin{tabular}{|c|c|c|c|c|c|}
\hline Zeit & & & arstposten $\left.^{1}\right)$ & & \\
\hline (Tage) & 1 & 2 & 3 & 4 & 5 \\
\hline Salmonell & azahl & & & & \\
\hline $\left.\begin{array}{ll}0 & \mathrm{~S} 1^{1}\end{array}\right)$ & $0,5 \cdot 10^{3}$ & $0,3 \cdot 10^{3}$ & $4,0 \cdot 10^{3}$ & $5,0 \cdot 10^{3}$ & $1,2 \cdot 10^{3}$ \\
\hline S2 & $6,0 \cdot 10^{4}$ & $6,3 \cdot 10^{4}$ & $5,4 \cdot 10^{4}$ & $7,5 \cdot 10^{4}$ & $7,0 \cdot 10^{4}$ \\
\hline 3 & $<100(r+)^{3}$ & $<100(\mathrm{r}+)$ & $0,6 \cdot 10^{3}$ & $1,9 \cdot 10^{3}$ & $6,4 \cdot 10^{3}$ \\
\hline & $8,8 \cdot 10^{4}$ & V & $2,8 \cdot 10^{4}$ & $2,1 \cdot 10^{4}$ & $8,1 \cdot 10^{3}$ \\
\hline 7 & $<100(\mathrm{r}+)$ & $<100(\mathrm{r}+)$ & $0,4 \cdot 10^{3}$ & $1,6 \cdot 10^{3}$ & $1,0 \cdot 10^{3}$ \\
\hline & $2,8 \cdot 10^{3}$ & $1,9 \cdot 10^{3}$ & $7,0 \cdot 10^{3}$ & $1,0 \cdot 10^{4}$ & $1,0 \cdot 10^{4}$ \\
\hline 14 & $<100(\mathrm{r}+)$ & $<100(\mathrm{r}+)$ & $0,1 \cdot 10^{3}$ & $0,4 \cdot 10^{3}$ & $0,1 \cdot 10^{3}$ \\
\hline & $2,5 \cdot 10^{3}$ & $<100(\mathrm{r}+)$ & $5,0 \cdot 10^{3}$ & $4,0 \cdot 10^{3}$ & $4,3 \cdot 10^{3}$ \\
\hline 21 & $<100(\mathrm{r}-)$ & $<100(\mathrm{r}-)$ & $0,1 \cdot 10^{3}$ & $0,2 \cdot 10^{3}$ & $0,1 \cdot 10^{3}$ \\
\hline & $1,2 \cdot 10^{3}$ & $<100(\mathrm{r}+)$ & $2,2 \cdot 10^{3}$ & $1,9 \cdot 10^{3}$ & $4,1 \cdot 10^{3}$ \\
\hline pH-Wert & & & & & \\
\hline 0 & 5,45 & 5,57 & 5,95 & 6,05 & 5,90 \\
\hline & 5,20 & 5,25 & 5,60 & 5,75 & 5,75 \\
\hline 3 & 5,20 & 5,30 & 5,60 & 5,70 & 5,75 \\
\hline & 5,20 & 5,25 & 5,25 & 5,60 & 5,60 \\
\hline 7 & 5,40 & 5,45 & 5,40 & 5,50 & 5,30 \\
\hline & 5,20 & 5,25 & 5,20 & 5,20 & 5,20 \\
\hline 14 & 5,10 & 4,90 & 5,10 & 4,95 & 4,95 \\
\hline & 4,85 & 4,80 & 5,00 & 5,25 & 5,00 \\
\hline 21 & 4,90 & 4,80 & 4,80 & 4,95 & 4,85 \\
\hline & 4,85 & 4,80 & 5,00 & 5,15 & 5,00 \\
\hline
\end{tabular}

1) Siehe Tabelle 5

2) Versuchsreihe

3) Anreicherung, $\mathrm{r}+=$ Wachstum

$\mathrm{r}-=$ Kein Wachstum

stellbarkeitsgrenze (100 Keime/g) lagen, in der Anreicherung aber positiv waren, unter dem Logarithmenwert 1 (=10 Keime/g) eingetragen. In Bild 39 sind die Ergebnisse für beide Versuche als Mittelwerte wiedergegeben.

Bei den GdL-Würsten mit dem kleineren Salmonellenzusatz sank die Salmonellenzahl innerhalb drei Tagen auf unter $100 \mathrm{Keime} / \mathrm{g}$, jedoch war die Anreicherung von sieben Tagen alten Würsten immer noch positiv. Nach 14 Tagen ergaben auch die Anreicherungen beider GdL-Würste einen negativen Befund. Bei den Starterkultur-Würsten ging die Salmonellenzahl langsamer zurück und blieb nach 21 Tagen auf einem Niveau von etwa 100 Keimen/g. Im Hinblick auf die Salmonellenzahl war auch von untergeordneter Bedeutung, mit welcher Nitratkonzentration und ob mit einer Nitrit-Nitrat-Mischung gearbeitet wurde.

Beim Versuch mit dem grösseren Salmonellenzusatz unterschied sich lediglich die Wurst 2, die 200 ppm $\mathrm{NaNO}_{2}$ und $0,6 \%$ GdL enthielt, von den übrigen Würsten; ihre Salmonellenzahl sank unter die Feststellbarkeitsgrenze, in der Anreicherung ergab sie jedoch am 21. Tag noch einen positiven Befund. Bei der entsprechenden Wurst mit der niedrigeren Nitritkonzentration sowie 


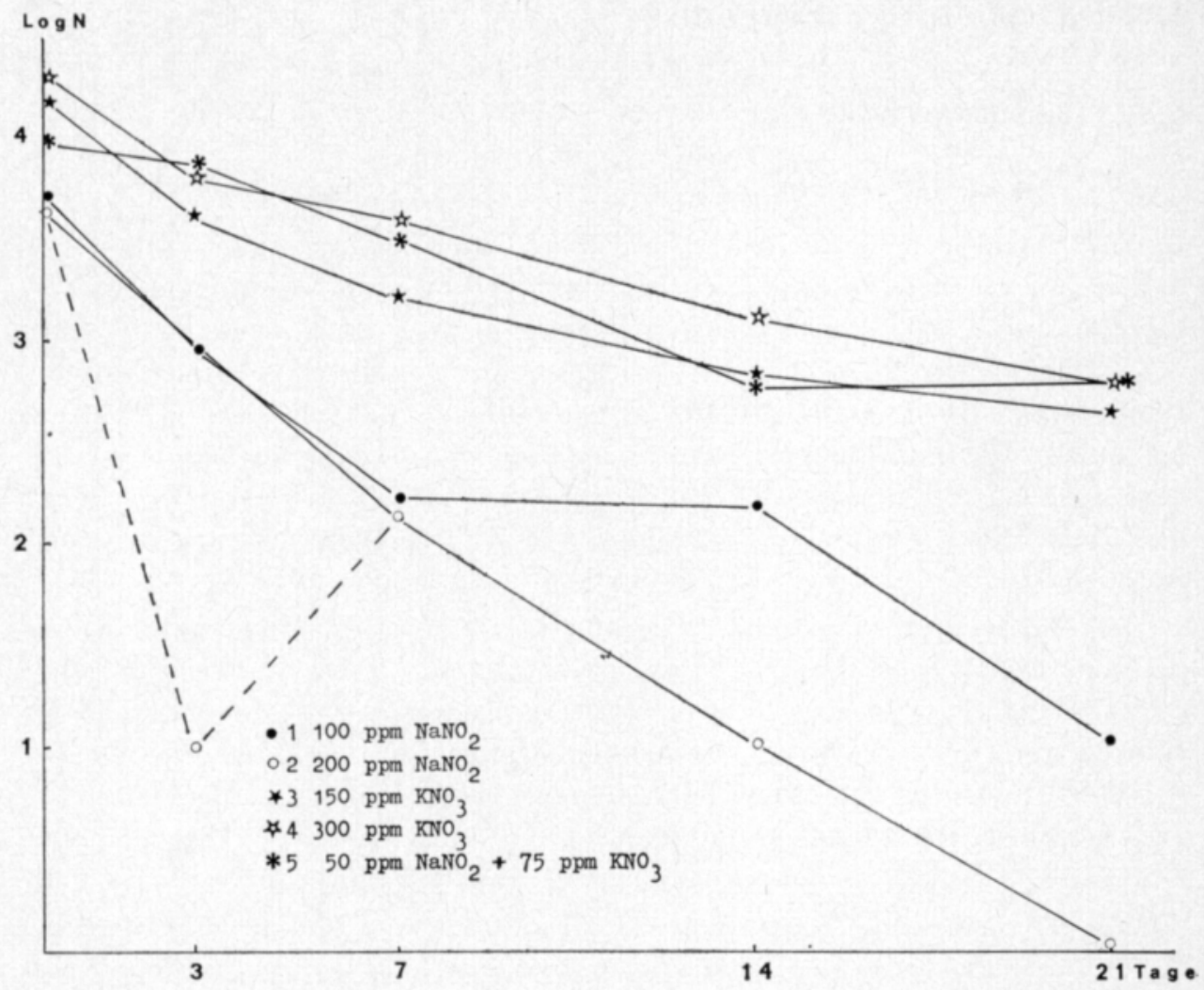

Bild 39. Anzahl an Salmonella senftenberg in den Salmonellenversuchsreihen S1 und S2; Mittelwerte.

bei sämtlichen Starterkultur-Würsten wurden ständig ziemlich hohe Salmonellenzahlen beobachtet. Zwischen den Würsten 1 und $3-5$ bestanden keine wesentlichen Unterschiede, jedoch hatte die GdL-Wurst vom siebten Tag an einen geringfügig niedrigeren Salmonellengehalt als die StarterkulturWürste.

In Tabelle 31 sind auch die im Zusammenhang mit den Salmonellenversuchen gemessenen $\mathrm{pH}$-Werte eingetragen. Anders als in dem Abschnitt 3. 5. 5 hatten die Starterkultur-Würste hier keine niedrigeren $\mathrm{pH}$-Werte als die GdL-Würste. Die Starterkultur-Würste hatten im ersten Versuch um 0,05-0,15, im zweiten Versuch um 0,15-0,35 Einheiten höhere pH-Werte als die GdL-Würste. 


\section{Diskussion der Ergebnisse}

\section{1 Präliminarversuche}

\section{Nitrit- und Nitratzusatz}

Die Versuche VII $-\mathrm{X}$ der vorliegenden Untersuchung ergaben, dass sich bereits mit einem sehr geringen Nitrit- bzw. Nitratzusatz $-25 \mathrm{ppm} \mathrm{NaNO}_{2}$ bzw. 50 ppm $\mathrm{KNO}_{3}-$ eine beständige Farbe erzielen lässt. Diese Feststellung wird durch frühere einschlägige Arbeiten untermauert (Fox und THOMsoN 1963, Mirna 1973, Wirth 1973, Kueper und Trelease 1974, Dethmers u.a. 1975). Die Umrötung ist hierbei jedoch nicht der kritische Faktor, sondern dem Arbeiten mit so niedrigen Konzentrationen steht der Umstand im Wege, dass dann die Rohwurst-Mikrobenflora eine Ảnderung erfährt und zu einem zu hohen gesundheitlichen Risiko wird (LEISTNER u.a. 1973 b).

Der Nitrit- und Nitratgehalt beeinflusst in erster Linie die Laktobazillentätigkeit (Bild 7 und 12), was sich wiederum auf den pH-Wert auswirkt (REUTER 1972). Bild 31 zeigt den im Versuch VIII ermittelten Einfluss des Nitritgehalts, Bild 32 den im Versuch VII ermittelten Einfluss des Nitratgehalts auf den $\mathrm{pH}-$ Wert. Wie aus den beiden Abbildungen hervorgeht, neigt der $\mathrm{pH}$ unterhalb der Konzentrationswerte $100 \mathrm{ppm} \mathrm{NaNO}$ und 125 ppm $\mathrm{KNO}_{3}$ zu raschem und tieferem Abfall als bei höheren Nitrit- und Nitratzusätzen.

Nach Nurmi und Turunen (1970) bewirkte eine Erhöhung des Nitritzusatzes von null auf $40 \mathrm{bzw}$. $200 \mathrm{ppm}$ nur eine verhältnismässig unbedeutende Verzögerung des Laktobazillen-Wachstums; andererseits hatte aber ein Nitritzusatz von $8 \mathrm{ppm}$ eine deutliche Zunahme des Wachstums zur Folge. Bei Erhöhung des Nitritzusatzes von 200 auf 1000 ppm wurde das Wachstum verlangsamt, und die definitive Laktobazillenzahl blieb deutlich auf einem niedrigeren Niveau. Bei einem Zusatz von 5000 ppm fand keine Vermehrung mehr statt. -Das Wachstum der heterofermentativen Laktobazillen wurde vom Nitrit stärker beeinflusst, denn die durch die 8 ppm bewirkte Wachstumszunahme und die durch die höheren Konzentrationen bewirkte Hemmung waren bei ihnen ausgeprägter als bei den homofermentativen Laktobazillen. Das Wachstum der Mikrokokken wurde durch 1000 bzw. 5000 ppm gehemmt, wohingegen die übrigen Konzentrationen das Wachstum nur geringfügig beeinflussten; ein Zusatz von 8 ppm führte zu vermehrtem Wachstum. Da die Säurebildung in Rohwurst homofermentativ (REUTER 1972) zu erfolgen hat, kann man davon ausgehen, dass zur Verhinderung von Geschmacksfehlern eine ausreichende Menge Nitrit (über $40 \mathrm{ppm}$ ) erforderlich ist. WIRTH (1973) hat denn auch beobachtet, dass Geschmacksfehler beim Arbeiten mit $30 \mathrm{ppm}$, nicht mehr aber beim Arbeiten mit 70 ppm $\mathrm{NaNO}_{2}$ auftraten.

Auch bezüglich der $\mathrm{pH}$-Abfallgeschwindigkeit zeigten die Würste sehr deutliche Unterschiede; diese waren am vierten Tag am ausgeprägtesten. Subjektiv betrachtet verlief die $\mathrm{pH}$-Abnahme bei den Würsten 1 und 2 (Bild 10) zu rasch, denn sie führte $\mathrm{zu}$ zu niedrigen pH-Werten (NIINIVAara und Ронја 1957), bei den Würsten 5-7 hingegen zu langsam, so dass sich die Würste, was die pathogenen Bakterien betrifft, ziemlich lange im Risikobereich 
befanden. Allerdings enthielten diese letztgenannten Würste reichliche Mengen Nitrit, wodurch die gesundheitliche Gefahr verringert wurde.

Bei Berücksichtigung aller Einzelversuche ist festzustellen, dass die $\mathrm{pH}$ Unterschiede zwischen den extrem kleinen und den üblichen Nitrit/Nitratzusätzen um so grösser werden, je stärker die Tendenz zu tiefem Absinken des $\mathrm{pH}$-Werts ist. Die Verwendung von Nitrit oder Nitrat ist besonders wichtig um Extremfälle auszuschliessen.

Nach Ninnivaara und PoHJa (1957) hat finnische Rohwurst normalerweise einen $\mathrm{pH}$ von 5,3; nach PoHJA und KnutaR (1975) variieren die pH-Mittelwerte je nach Sorte normalerweise zwischen 4,8 und 5,0 , die $\mathrm{pH}$-Werte von Einzelwürsten zwischen 4,5 und 5,3. In den Untersuchungen von NuRmi (1966 a und b) liegen die pH-Werte höher als von Pohja und Knutar angegeben, entsprechen aber den von Niinivaara und Pohja publizierten Werten. Die pH-Wert-Ergebnisse der vorliegenden Arbeit stimmen mit den Resultaten von Pohja und Knutar überein, was darauf hinweist, dass die durchschnittlichen $\mathrm{pH}-$ Werte in den vergangenen zehn Jahren geringfügig zurückgegangen sind. Der Grund für diese Erscheinung könnte in dem zunehmenden Einsatz von GdL und Starterkulturen sowie in der Erhöhung des Zuckerzusatzes zu suchen sein. Der pH-Wert der GdL-Würste stimmte im grossen und ganzen mit den Resultaten anderer entsprechender Untersuchungen überein (NURMI 1966 b, REuter 1972, SKJELKVÅle u.a. 1974).

Desgleichen entsprachen die Auswirkungen des pH-Werts im allgemeinen früheren einschlägigen Beobachtungen (NinnivaARA 1955, Nurmi 1966 a und b, Review: CoRetti 1975).

Der pH-Abfall erfolgte bei einem Nitritgehalt von 100 bzw. 200 ppm genügend schnell und kam auf dem richtigen Niveau zum Stillstand. pHWert-Betrachtungen können freilich im wesentlichen nur bezüglich der zwischen den Wurstposten der gleichen Versuchsreihe bestehenden Unterschiede angestellt werden, denn die $\mathrm{pH}$-Wert-Entwicklung verlief bei den einzelnen Versuchsreihen so unterschiedlich, dass eine auf quantitativ bescheidene Unterlagen gestützte Verallgemeinerung oft nicht möglich ist.

Aus den vorgenannten Gründen werden deshalb auf Grund der vorliegenden Untersuchung für den Nitritzusatz $100 \mathrm{ppm}$, für den Nitratzusatz $150 \mathrm{ppm}$ empfohlen. Diese Werte stehen im Einklang mit den entsprechenden Empfehlungen von WIRTH (1974).

Die zwischen Nitrit bzw. Nitrat und Restnitrit festgestellten Korrelationskoeffizienten $\mathrm{r}=+0,992\left(\mathrm{NaNO}_{2}\right)$ bzw. $\mathrm{r}=+0,991\left(\mathrm{KNO}_{3}\right)$ zeigten, dass die zugesetzte Nitritmenge entscheidend ist wenn es darum geht, den Restnitritgehalt zu senken. Diese Erkenntnis geht auch aus anderen entsprechenden Untersuchungen hervor (Wirth 1973, Gerhard und Haller 1973, Mirna 1973, Dethmers u.a. 1975, Nestorov u.a. 1975).

Die Abnahme der Nitritkonzentration erfolgte bei den hier durchgeführten Versuchen nicht die ganze Zeit über logarithmisch in der von NoRDIN (1969) dargelegten Weise. In Bild 40 ist die Nitritkonzentration in ein halblogarithmisches Koordinatensystem eingetragen; wie ersichtlich, nahm die Nitritmenge bei den höheren Nitritkonzentrationen relativ langsamer ab als bei den niedrigeren Konzentrationen. Dies dürfte darin begründet sein, dass die 


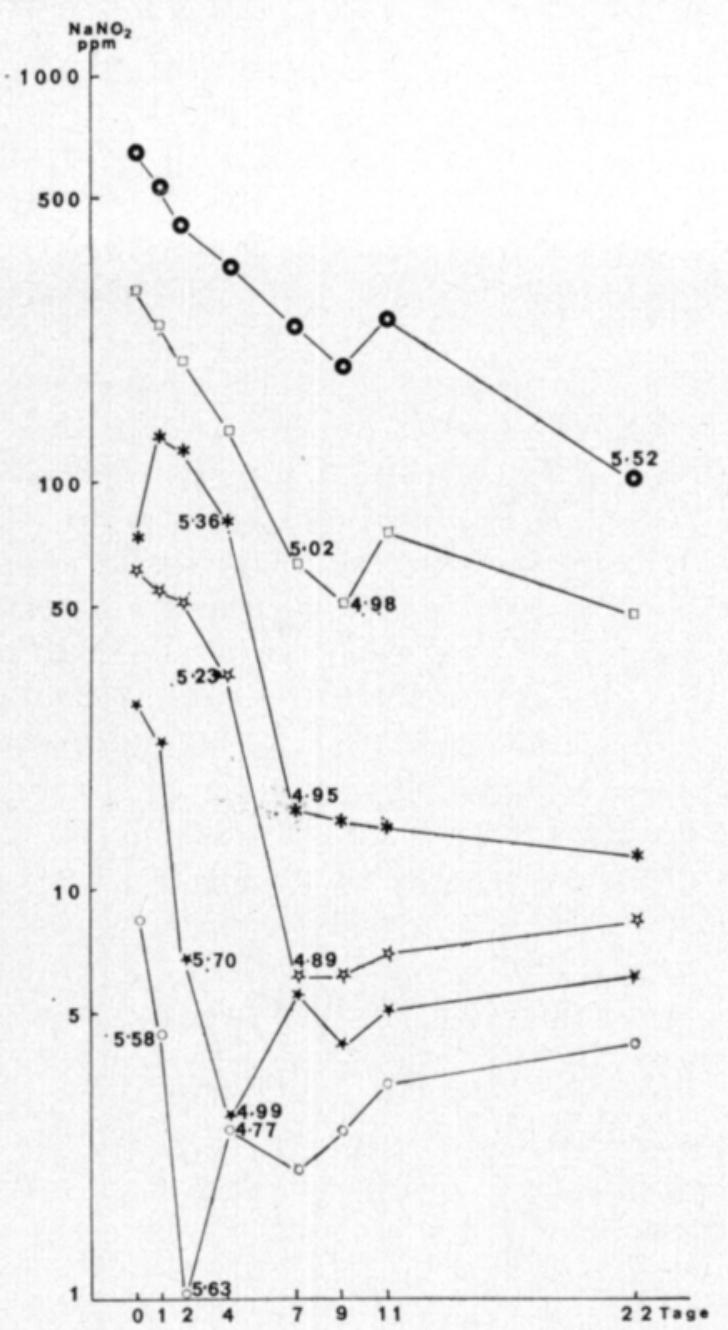

Bild 40. Nitritabnahme beim Arbeiten mit verschieden grossen $\mathrm{Ni}$ tritzusätzen. An den Knickstellen die zum gleichen Zeitpunkt festgestellten pH-Werte. Versuchsreihe VIII B.

Kapazität der im Fleisch enthaltenen, den Nitritverlust beeinflussenden Faktoren begrenzt ist. Der $\mathrm{pH}$ sank schneller in den Würsten, die weniger Nitrit enthielten. Dies wiederum bewirkte einen Nitritabbau, der jedoch in der Regel etwa beim Passieren von $\mathrm{pH} \mathrm{5,0}$ zum Stillstand kam. Auch in anderen Versuchsreihen (z. B. vergleichende Versuchsreihen, Bild 36 und 38) bildete die Nitritgehaltskurve bei etwa $\mathrm{pH}$ 5,0 einen Knick, eine Erscheinung, die auch Pfeil und Liepe (1974) bemerkt haben.

Die Ursache des aus Bild 40 ersichtlichen Einflusses des pH-Werts auf den Rückgang des Nitritgehalts blieb ungeklärt. Das Aussetzen der Nitritabnahme kann etwa durch Hemmung der nitritabbauenden Faktoren oder dadurch bedingt sein, dass das Nitrit mit sinkendem $\mathrm{pH}$ so fest an die Proteine gebunden wird (Mrrna 1971), dass kein Abbau mehr erfolgt. Infolge des niedrigen $\mathrm{pH}-$ Werts werden dann auch keine nennenswerten Nitratmengen mehr reduziert (siehe hierzu folgenden Abschnitt). Der Zeit kam in dieser Beziehung keine entscheidende Bedeutung zu, denn der Tag, an dem die Knickstelle erreicht wurde, war von Wurst zu Wurst verschieden. 
Die Kurve der Nitratreduktion zeigte im $\mathrm{pH}$-Bereich von 5,2-5,3 einen Knick (Bild 41); hinter diesem verlief die Reduktion dann nur langsam. Die Knickstelle lag um ein geringes unter dem von NIINIvAARA (1955) angegebenen pH-Wert 5,4. Die Differenz kann zum einen auf Unterschiede in der pHMessung, zum anderen aber auch darauf zurückzuführen sein, dass in der vor-

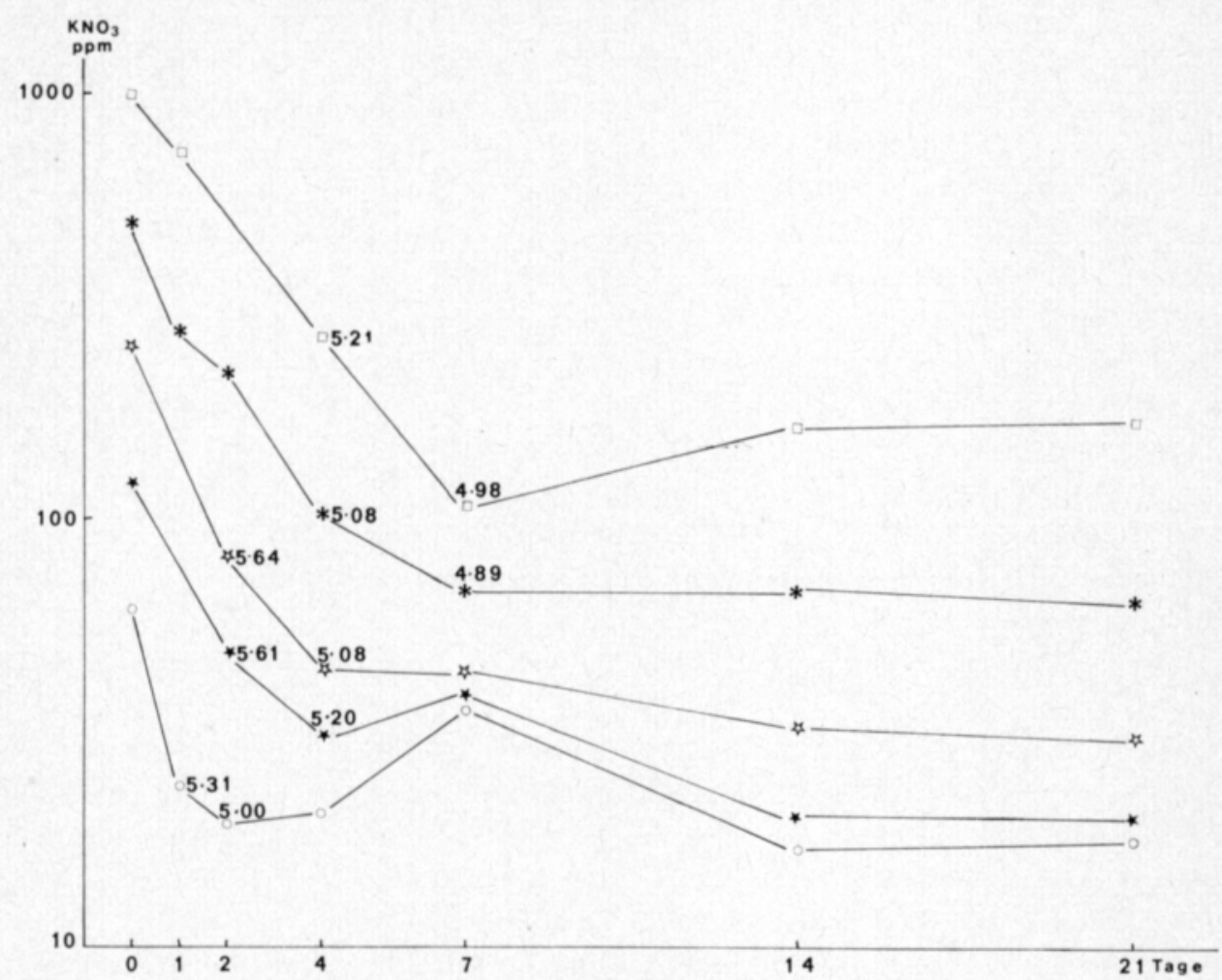

Bild 41. Nitratabnahme beim Arbeiten mit verschieden grossen Nitratzusätzen. An den Knickstellen die zum gleichen Zeitpunkt festgestellten -pH-Werte. Versuchsreihe VIII B.

liegenden Arbeit der Knickpunkt zwischen zwei aufeinanderfolgenden Stichtagen interpoliert wurde. Bei entsprechender Verfahrensweise liefern auch die Resultate der von Niinivaara durchgeführten Bouillon- und Wurstversuche einen etwas niedrigeren Wert. Die Nitratreduktase der Mikrokokken hingegen wäre selbst bei noch niedrigeren $\mathrm{pH}-$ Werten weiterhin in hohem Grade wirksam (Puolanne u.a. 1977), so dass die Gründe für das Abklingen der Reduktion an anderer Stelle zu suchen sind.

Nach heutiger Erkenntnis muss der Nitrit- bzw. Nitratzusatz, wenn schon nicht ganz auf ihn verzichtet werden kann, so gering wie möglich gehalten werden. Dabei ist namentlich die zugesetzte Nitrit- und Nitratmenge von Bedeutung, denn wir wissen heute noch nicht genau, in welche Verbindungen das Nitrit bzw. Nitrat in den Fleischwaren umgewandelt wird. Die auf Grund der Analyse festgestellte Menge an freiem oder gebundenem Nitrit vermittelt somit nur ein partielles Bild des durch Synthese von N-Nitrosoverbindungen 
in den Fleischwaren oder in vivo gegebenen Risikos. Man kann also zumindest annehmen, dass auch der Teil des Nitrits bzw. Nitrats, der in der Analyse als sverschwunden" ausgewiesen wird, später bei der Bildung von N-Nitrosoverbindungen ein Gefahrenmoment darstellt (Proc. ISNMP 2. 1977).

St arterkulturen und GdL

Mit Starterkulturen wurden niedrigere Restnitrit- und -nitratmengen als ohne erzielt (Bild 18); zu dieser Feststellung sind auch NinnvaARA (1955), Pfeil und Liepe (1973b), Nagata und Mirna (1974) sowie Kotter u.a. (1976) gelangt. GdL-Zusatz lieferte einen niedrigeren Restnitritgehalt, wohingegen der Nitratgehalt höher als bei den entsprechenden Starterkultur-Würsten lag. Analoge Ergebnisse wurden auch von SAIr (1963), Meester (1965), Wirth (1973) und Kotter u.a. (1976) publiziert. Der pH-Wert der GdL-Würste sinkt schneller als der der Starterkultur-Würste. PfeIL und LIEPE (1974) konstatierten, dass der $\mathrm{pH}$-Wert nicht zu schnell sinken darf, weil dann die Tätigkeit der nitrit- und nitratreduzierenden Bakterien langsamer wird, und die Restnitrit- und -nitratgehalte höher bleiben. Bei Verwendung von Starterkulturen kann der Nitrit- oder Nitratzusatz verringert werden, weil mit optimaler $\mathrm{pH}$-Abfallgeschwindigkeit die Zusatzstoffe vollkommener verwertet werden können.

Nach NoRdin (1969) erfährt der Nitritschwund mit abnehmendem pH eine Beschleunigung. Bild 40 lässt die gleiche Folgerung $\mathrm{zu}$, denn mit abnehmendem Nitritzusatz stieg die $\mathrm{pH}$-Abnahmegeschwindigkeit und damit gleichzeitig die relative Nitrit-Abbaugeschwindigkeit. Diese Erscheinung war bis herunter zu $\mathrm{pH} \mathrm{5,0} \mathrm{zu} \mathrm{beobachten.} \mathrm{In} \mathrm{der} \mathrm{Versuchsreihe} \mathrm{XII} \mathrm{hatten} \mathrm{die} \mathrm{mit}$ Mikrokokken-Laktobazillen-Starterkulturen hergestellten Würste bei Versuchsende einen geringeren Nitritgehalt als die ausschliesslich mit MikrokokkenStarterkultur hergestellten Würste, was namentlich darauf zurückgeführt werden kann, dass die Mikrokokken-Laktobazillen-Starterkulturen enthaltenden Würste zum Schluss den niedrigeren pH-Wert aufwiesen. Eine analoge Erscheinung hat auch PETÄJÄ (1977) festgestellt.

Die Starterkultur-Konzentration zeigte in der Versuchsreihe XI nur verhältnismässig bescheidene Auswirkungen auf die Nitritmenge (Bild 18), wohingegen die Nitratmenge mit steigender Starterkultur-Konzentration deutlich abnahm (Bild 19). Die Vorbehandlungsart der Starterkulturen blieb hingegen in der Versuchsreihe XII ohne Einfluss; die Unterschiede wurden hauptsächlich zwischen den Mikrokokken- und Mikrokokken-Laktobazillen-Würsten sichtbar. Wesentliche Differenzen wurden dabei beim Nitrit- (Bild 20) und Nitratgehalt, beim $\mathrm{pH}$-Wert und bei der Farbe festgestellt. Es besteht jedoch Grund zu der Annahme, dass durch Arbeiten mit aktivem Material (flying start) der $\mathrm{pH}$ etwas schneller gesenkt und die Restnitritmenge verringert werden kann. NURMi (1966 a) hat ferner konstatiert, dass bei Zusatz von Vegetativzellen der $\mathrm{pH}$-Wert etwas schneller sinkt als beim Arbeiten mit lyophilisierten Zellen. 
Durch Zusetzen von Askorbinverbindungen wird die Restnitritmenge gesenkt und die Umrötung gefördert (Möhler 1959, Mirna 1970, Wirth 1973, Kotter u.a. 1976), die Restnitratmenge jedoch erhöht (WIRTH 1973). Die vorliegende Untersuchung lieferte gleichartige Ergebnisse (Bild 21 und 22), wobei allerdings eine Beschleunigung der Umrötung zu Beginn des Herstellungsprozesses erfolgte. Mit Erythorbat wurde eine geringfügige Abnahme der Bakterienzahl erzielt - eine Erscheinung, die auch im Zusammenhang mit Brühwurst beobachtet worden ist (Nurmi u.a. 1975, RAEvuori 1975).

\section{Z u c k e r}

TÄNDLER (1963) empfiehlt auf Grund einer umfangreichen FachliteraturAuswertung für den Zuckergehalt eine Obergrenze von 0,3\%. Die Ausgangsbasis für diesen Richtwert war allerdings das Arbeiten mit grossen Nitratzusätzen ohne Starterkultur und zu einer Zeit, da Farbfehler gang und gäbe waren. CoRETti und TÄNDLER (1965) stellten fest, dass Zucker bis zu einem Gehalt von $0,6 \%$ die Eigenschaften der Wurst verbesserte, dass es aber bei noch stärkeren Zusätzen u.U. zu einem zu tiefen Absinken des pH-Werts kam. In der vorliegenden Untersuchung wurde durch Glukosekonzentrationen über $0,4 \%$ kein weiterer $\mathrm{pH}$-Abfall erzielt (Bild 35), d.h. es bestand also keine völlige Úbereinstimmung mit dem von CORETTI (1975) dargelegten Resultat. Die Versuchsreihe lieferte insofern ungewöhnliche Ergebnisse, als einerseits die ohne Glukosezusatz hergestellten Würste einen so niedrigen $\mathrm{pH}$ wie $\mathbf{5 , 1}$ erreichten, andererseits aber der $\mathrm{pH}$-Wert der $0,4-0,8 \%$ Glukose enthaltenden Würste auf etwa 4,7 stehenblieb. In einzelnen Fällen wurde im Rahmen dieser Arbeit mit 0,5\% Glukosezusatz ein $\mathrm{pH}$ von 4,4 erreicht. In der betreffenden Versuchsreihe war mit ziemlich hohem Nitritzusatz (150 ppm) gearbeitet worden, wodurch eventuell - auch wenn genügend Glukose vorhanden war - ein sehr tiefes Absinken des $\mathrm{pH}$-Werts verhindert wurde.

Die $\mathrm{pH}$-Werte variierten zwar in dieser Versuchsreihe sehr stark, jedoch enthielten alle Würste die gleichen Restnitrit- und -nitratmengen. Auch erreichten sämtliche Würste ungefähr zur gleichen Zeit einen $\mathrm{pH}$ von etwa 5,0 (Bild 29). Der pH-Wert der Wurst mit 0,4\% Glukosegehalt sank, verglichen an den übrigen Würsten, etwas rascher; ausserdem hatte sie einen etwas höheren Nitritgehalt. Andererseits fiel der $\mathrm{pH}$ der zuckerfreien Wurst nicht bis auf 5,0, ihr Nitritgehalt entsprach aber durchaus dem der anderen Würste.

T e m perat ur

Der Temperatureinfluss auf den Nitrit- und Nitratgehalt der Rohwurst ist noch weitgehend ungeklärt. Hingegen ist bekannt, dass Temperaturerhöhung auf $26^{\circ} \mathrm{C}$ im Zusammenhang mit Nitrat die Farbfehler-Gefahr erhöht, während sie beim Arbeiten mit Nitrit nicht im gleichen Masse gegeben scheint. Bei $18^{\circ} \mathrm{C}$ wird eine bessere Farbe erzielt, jedoch nimmt die Umrötung mehr Zeit in Anspruch (CORETTI und TÄNDLER 1965). In der vorliegenden Arbeit wurden 
keine temperaturbedingten Unterschiede zwischen Nitrit- und Nitratwirkung festgestellt, was auf den Zusatz von Starterkulturen zurückzuführen sein könnte, auf den CoRETTI und TäNDLER (1965) in ihrer Untersuchung verzichteten. Beim hier zur Diskussion stehenden Versuch entsprachen die Nitritgehaltsänderungen der Nitrat-Würste den anderweitig dargelegten Resultaten (Puolanne u.a. 1977). Bei der höchsten gefahrenen Temperatur wurde das - gleichzeitig auch grösste - Maximum am schnellsten erreicht; mit abnehmender Temperatur verlor das Maximum an Höhe und wurde mit Verzögerung erreicht. Auch hier könnte der Umstand, dass bei höherer Temperatur pH 5,0 früher erreicht wurde als bei niedrigeren Temperaturen, der Grund dafür sein, dass sich der Restnitritgehalt sämtlicher Würste auf das gleiche Niveau einspielte (Bild 25-27).

Auf den $\mathrm{pH}$ wirkte sich die Temperatur in der aus Bild 29 und 30 hervorgehenden Weise aus. Die pH-Wert-Entwicklung entsprach, was die NitritWürste betrifft, den Ergebnissen von CORETTI und TÄNDLER (1965). Darüber hinaus wurden aber - anders als in der Untersuchung von Coretti - auch bei Zusatz von Nitrat analoge Resultate erzielt, und zwar ohne dass sich bei höheren Temperaturen irgendwelche schädlichen Wirkungen zeigten.

\section{Alter des Fleisches}

Der in mikrobiologischer Hinsicht bedeutsamste Unterschied war der hohe Hefezellengehalt der älteren Fleischpartien. In bezug auf Nitrat, Nitrit und den $\mathrm{pH}$ wurden keine Unterschiede von Bedeutung festgestellt. Die beste Umrötung wurde mit dem frischesten Fleisch erzielt.

Durch Reifung des Fleisches wurden somit keinerlei Vorteile erzielt, wohl aber ergaben sich daraus Nachteile. Ein Geschmackstest, der vielleicht zu einem anderen Resultat geführt hätte, wurde nicht durchgeführt.

Zur Feststellung des Nitrosamin-Risikos wäre ein Analysieren der Amine erforderlich gewesen (VANDEKERKCHOEve und DEMEYer 1976).

\section{2 Vergleichende Versuche}

In diesem Zusammenhang wird auf eine Wiederholung der im Abschnitt 3.5. gemachten Feststellungen verzichtet; die Gegenüberstellung erfolgt hier vielmehr durch Vergleich der Würste beider Gruppen (d.h. also GdL-Würste und Starterkultur-Würste) innerhalb der Gruppe sowie von Gruppe zu Gruppe.

Bei den GdL-Würsten bewirkte eine Senkung des Nitritzusatzes von 200 auf $100 \mathrm{ppm}$ eine Restnitritabnahme von etwa 15 auf etwa $10 \mathrm{ppm}$ (Tabelle 15). Bei den Starterkultur-Würsten hatte die Verringerung des Nitratzusatzes von 300 auf $150 \mathrm{ppm}$ einen Rückgang der Restnitritmenge von etwa 13 auf etwa $10 \mathrm{ppm}$ zur Folge. Der Nitratgehalt lag bei den GdL-Würsten allerdings sehr hoch - am 14. Tag ca. $100 \mathrm{ppm}$ - und wurde auch durch Verringerung der Nitritmenge nicht beeinflusst (Tabelle 16). Aus Tabelle 17 geht hervor, dass die wesentlichen Änderungen des Nitrit- und Nitratgehalts bereits innerhalb des ersten Tages erfolgten, wobei auch der $\mathrm{pH}$ seinen Endwert erreichte (Tabelle 22 und Bild 38). 
In den Starterkultur-Würsten hielt der Nitratrückgang bis zum siebten Tag an. Durch Verringerung des Nitratzusatzes wurde eine deutliche Abnahme des Restnitratgehalts erzielt. Besonders augenfällig war der Unterschied zwischen GdL- und Starterkultur-Würsten nach Bild 37 und Tabelle 16, aus denen hervorgeht, dass die GdL-Würste eindeutig mehr Nitrat und damit einen beträchtlich höheren Gesamtrestgehalt an Nitrit und Nitrat aufwiesen (Tabelle 17).

Bei den Stickoxidmyoglobin-Mengen wurden am dritten Tag keine signifikanten Unterschiede festgestellt; am 14. Tag enthielten die StarterkulturWürste jedoch signifikant mehr Stickoxidmyoglobin als die nitritärmere GdLWurst (Tabelle 18). Die Unterschiede zwischen den GdL-Würsten zeigten den gleichen Trend wie in den Untersuchungen von GERHARD und HALLER (1973), Mirna (1973) sowie Kotter u.a. (1976), in denen jedoch keine Vergleiche der hier beschriebenen Art zwischen GdL- und Starterkultur-Würsten angestellt worden waren. Nach Kotter u.a. (1976) lag bei Zusatz von Nitrit und einer Askorbinverbindung der relative Stickoxidmyoglobin-Anteil bei den GdL-Würsten höher als bei der Starterkultur-Wurst. Die vorliegende Untersuchung ergab freilich in Ubereinstimmung mit einer Arbeit von Nestorov u.a. (1975), dass eine Senkung des Nitrit- bzw. Nitratgehalts keinen signifikanten Einfluss auf die Stickoxidmyoglobin-Menge hat.

Die Bakterienzahl wurde durch Senkung des Nitrit- und Nitratzusatzes nicht beeinflusst (Tabelle 19-20). In den Präliminarversuchen wurde mit steigendem Nitrit- bzw. Nitratzusatz eine Verlangsamung oder sogar Hemmung des Laktobazillen-Wachstums festgestellt. Am deutlichsten war diese Erscheinung bei vom üblichen abweichendem Zusatz zu beobachten (Bild 7 und 12). Die beim Arbeiten mit allgemein üblichen Nitrit- bzw. Nitratzusätzen ermittelten Laktobazillen-Zahlen entsprechen etwa den Resultaten anderer Forscher (Nurmi $1966 \mathrm{a}$ und b, Reuter 1972, SkJelkvåle u.a. 1974). In den vergleichenden Versuchsreihen zeigten die Laktobazillen in den GdLWürsten eine normale Wachstumsphase (Nurmi 1966 b, REUTER 1972), während bei den Starterkulturen keine Vermehrung erfolgte. Eine Verringerung des Nitrit- bzw. Nitratzusatzes hatte jedoch keinen Einfluss auf das Wachstum der Laktobazillen; dies war auf Grund der Präliminarversuche bei diesen Zusatzmittelgehalten zu erwarten.

Nưrmi (1966 a) und Reuter (1972) konstatierten, dass die in Form von Starterkulturen zugesetzten Mikrokokken zahlenmässig zunächst zunahmen und dann langsam zurückgingen. In der vorliegenden Untersuchung (Bild 8, Tabelle 21), die Präliminarversuche inbegriffen, zeigten die Mikrokokken anfangs nur sehr schwaches oder überhaupt kein Wachstum und anschliessend einen steileren Abfall als von Nurmi (1966 a) und Reuter (1972) dargelegt. Die Quantität des Nitrit- bzw. Nitratzusatzes blieb ohne Auswirkungen auf die Mikrokokkenzahl (Tabelle 21).

Die fäkalen Streptokokken lagen während der gesamten Versuchsdauer zahlenmässig deutlich niedriger (im allgemeinen unter $1000 \mathrm{Keime} / \mathrm{g}$ ) als in der Untersuchung von Nurmi (1966 a) $\left(10^{5}-10^{6} \mathrm{Keime} / \mathrm{g}\right)$; allerdings operierte dieser im allgemeinen mit höheren $\mathrm{pH}$-Werten. Bei einer Charge mit sehr 
hohem Laktobazillengehalt stellte auch Nurmi nur einen Gehalt von etwa $10^{3}$ fäkalen Streptokokken pro Gramm fest.

Die Anzahl der Hefezellen blieb während der gesamten Versuchsdauer niedrig, und in den vergleichenden Versuchen wurde auch keine Beeinflussung derselben durch die Nitrit- und/oder Nitratmenge beobachtet. REUTER (1972) hat in seiner Untersuchung festgestellt, dass die Hefen sich in den GdL-Würsten sehr stark vermehren können, in der vorliegenden Arbeit aber blieb die Vermehrung der Hefen unbedeutend.

Nach SKJELKVÅle u.a. (1973) hat selbst ein völliges Weglassen des Nitrits keine Auswirkungen auf die Bakterienzahl, es sei denn, man arbeitet mit einer Nitrit-Starterkultur-Kombination. Die Starterkultur-Würste mit Nitrit enthielten weniger Laktobazillen als die Starterkultur-Würste ohne Nitrit. In den Präliminarversuchen wurden deutliche Unterschiede erst beim Arbeiten mit niedrigeren Nitrit- und Nitratzusätzen als in den vergleichenden Versuchen beobachtet. Die Ergebnisse der vorliegenden Arbeit decken sich am ehesten mit den Resultaten einer Untersuchung von Leistner u.a. (1973 b), in der sie konstatieren, dass eine Verringerung des Nitritzusatzes um $50 \%$ nicht unbedingt nachteilige Veränderungen der Gesamtbakterienzahl zur Folge hat, dass aber bei Weglassen des Nitrits die Gesamtbakterienzahl auf etwa $10^{9}$ Keime/g steigt.

Der $\mathrm{pH}$-Wert ist in diesem Zusammenhang die bedeutendste physikalische Grösse, und die übrigen Faktoren folgen seiner Entwicklung mehr oder weniger. Bei den GdL-Würsten sank der $\mathrm{pH}$ bereits innerhalb des ersten Tages nahezu auf seinen Endwert, und zwar in beiden Würsten mit der gleichen Geschwindigkeit. Die stärker nitrithaltige Wurst hatte allerdings nach dem ersten Tag einen etwas höheren $\mathrm{pH}$-Wert als die nitritärmere Wurst (Tabelle 22 und Bild 38).

Bei den Starterkultur-Würsten verlief die $\mathrm{pH}$-Abnahme langsamer als bei den GdL-Würsten, wobei die stärker nitrathaltige Wurst ständig einen etwas höheren $\mathrm{pH}$ als die übrigen beiden Starterkultur-Würste hatte; der Unterschied war jedoch unbedeutend. Die GdL-Würste hatten einen beträchtlich höheren End-pH als die Starterkultur-Würste. Die Temperatur wurde in den ersten beiden Tagen des Versuchs auf $22^{\circ} \mathrm{C}$ gehalten, was vielleicht einer der Gründe für den niedrigen End-pH der Starterkultur-Würste war.

Der Einfluss der Nitritkonzentration zeigte sich in der Farbe der GdLWürste. Die mit höherem Nitritzusatz hergestellte Wurst hatte ständig eine kräftigere Oberflächenfarbe als die Wurst mit dem niedrigeren Nitritzusatz; beim Schnittbild der fertigen Würste waren jedoch keine Farbunterschiede festzustellen. Offensichtlich bilden GdL und Askorbinsäure zusammen mit dem Luftsauerstoff ein Milieu, in dem bei niedrigem Nitritgehalt keine Umrötung stattfindet. Es ist ferner anzunehmen, dass an der Wurstoberfläche die gasförmigen Zerfallsprodukte des Nitrits rasch verdunsten. Nach BRooks (1938) nimmt mit sinkendem $\mathrm{pH}$ auch die Metmyoglobinbildung zu. Andererseits wird aber mit abnehmendem $\mathrm{pH}$ Stickoxid in verstärktem Masse gebunden (Mrrna 1971). Der pH-Wert sinkt bei GdL-Wurst rasch. Bei dieser Bindung an das Myoglobin handelt es sich um eine sehr schnelle Reaktion (BRooks 1938), die mit abnehmendem $\mathrm{pH}$ noch beschleunigt wird. 
Hinsichtlich Oberflächen- und Schnittflächenfarbe zeigten die Starterkultur-Würste erst vom 22. Tag an gegenseitige Unterschiede; von besagtem Tag an hatte die mit Nitrit-Nitrat-Zusatz hergestellte Wurst die beste Farbe.

Die Starterkultur-Würste waren, was die Farbbeständigkeit anbelangt, den GdL-Würsten überlegen, was auf den niedrigen $\mathrm{pH}$-Wert der ersteren zurückzuführen sein dürfte (NirnivaARa 1955). Der Nitrit- bzw. Nitratgehalt war in dieser Beziehung ohne Einfluss.

Diẹ Ergebnisse dieser Untersuchung finden ihre Bestätigung durch die von Wirth (1973) gemachte Beobachtung, dass 20-30 ppm Nitrit für eine gleichmässige Umrötung der Rohwurst ausreichen. Auch Dethmers u.a. (1975) haben beim Arbeiten mit 50 ppm oder mehr Nitrit keine Farbverbesserung festgestellt.

Bei gleichzeitiger Verwendung von Starterkulturen und Nitrat wies das Schnittbild der Wurst oft einen Stich ins Bräunliche auf. Diese Erscheinung war wahrscheinlich auf die Bildung von Metmyoglobinnitrit zurückzuführen (Fox und Thomson 1963), denn bei hohem $\mathrm{pH}$ kam es zur Bildung reichlicher Nitritmengen, die keine Verbindung mit den Proteinen eingingen. Mit abnehmendem $\mathrm{pH}$ verschwand diese bräunliche Färbung.

Die zugesetzte Nitrit- bzw. Nitratmenge beeinflusste in gewissem Grade die geschmacklichen Eigenschaften der Würste. Die mit dem höheren Nitrit- bzw. Nitratzusatz hergestellten Würste erzielten bei der Beurteilung etwas höhere Punktzahlen als die Würste mit dem geringeren Zusatz; die Unterschiede waren jedoch gering und statistisch nicht signifikant. Am besten schnitt in dieser Beziehung die Wurst mit Nitrit-Nitrat-Zusatz ab, obwohl die Gesamtmenge dieser beiden Stoffe dem niedrigeren Zusatz entsprach. Diese Kombination entspricht der in den USA verwendeten Nitrit-Nitrat-Mischung 1: 1, die im Verhältnis 1:99 mit Kochsalz vermengt ("Prague Powder») zugesetzt wird. Nagata und Mirna (1974) empfehlen eine aus 2/3 Nitrit und 1/3 Nitrat zusammengesetzte Mischung, da hierbei das Gleichgewicht zwischen Nitrit, Nitrat, Myoglobin und Metmyoglobin in der Form erzielt wird, dass sich weniger Nitrit über Myoglobin-Oxydation in Nitrat umwandelt.

Die Versuchsdauer, 14 Tage, war vielleicht für Geschmacksuntersuchungen im Zusammenhang mit dem Vergleich zwischen Starterkultur- und GdLWürsten doch zu kurz, jedoch bildeten derartige Untersuchungen auch nicht den Zweck der vorliegenden Arbeit.

\section{3 Salmonellenversuche}

Um festzustellen, in welchem Masse sich eine Senkung der Nitrit- bzw. Nitratkonzentration auf die Vegetationsmöglichkeiten von pathogenen Bakterien in der Rohwurst auswirkt, wurde das Wachstum von Salmonella senftenberg unter den bei den vergleichenden Versuchen angewandten Zusatzmittel-Alternativen untersucht. Bereits früher wurde in Finnland die Feststellung gemacht, dass Starterkulturen die Toxinbildung von Staphylococcus aureus in Rohwurst weitgehend hemmen (Niskanen und Nurmi 1976), und nach RaEvuori und GENIGEORGIS (1975) wird durch Senkung des pH-Werts unter den Neutralpunkt und Erhöhung der Kochsalzkonzentration das Wachstum von Bacillus 
cereus verhindert. Weiter verzögern Nitrit und Erythorbat das Wachstum von B. cereus (RAEvUoRI 1975), so dass B. cereus in der Rohwurst keinen wirklichen Risikofaktor darstellt. Die pathogenen Vertreter der Gattung Clostridium vermehren sich in Rohwurst nicht (NuRMi 1975, mündliche Information, Roberts und Ingram 1977). In Anbetracht dieser Tatsache konnte vorausgesetzt werden, dass sich durch Untersuchung des Salmonellen-Wachstums die bei Verringerung der Nitrit- und Nitratmengen durch pathogene Bakterien bedingte Risikozunahme im wesentlichen erfassen lässt.

Die Salmonellenversuche (Tabelle 31 und Bild 39) machten deutlich, dass eine Senkung der Nitrit- bzw. Nitratmenge auf die Hälfte des gegenwärtig üblichen Quantums kein erhöhtes gesundheitliches Risiko durch Salmonellen zur Folge hat.

Ausserdem lag der $\mathrm{pH}$ bei den GdL-Würsten niedriger, bei den Starterkultur-Würsten jedoch höher (Tabelle 31) als bei den entsprechenden Würsten der vergleichenden Versuche (Tabelle 22 und Bild 18). Da - abgesehen vom Kuttern - die Würste weder alle an der gleichen Stelle hergestellt noch analysiert worden waren, liess sich der Grund für diese Abweichung nicht feststellen. Vorausgesetzt, es handelt sich um einen tatsächlichen, nicht auf die Bestimmungsart zurückzuführenden Unterschied, so sind damit die Änderungen der Salmonellenzahl zu einem wesentlichen Teil erklärt, denn man hat festgestellt, dass die pH-Abnahme einen entscheidenden Einfluss auf die Vermehrung bzw. das Absterben der Salmonellen hat (SIRviö u.a. 1977).

An Hand der Salmonellenversuche konnte somit der Beweis erbracht werden, dass eine Senkung der Nitrit- bzw. Nitratmenge auf die Hälfte des gegenwärtig üblichen Wertes keine Erhöhung des durch Salmonellen bedingten gesundheitlichen Risikos bedeutet, denn der bestimmende Faktor im Hinblick auf die Hemmung des Salmonellen-Wachstums ist der pH-Wert. Diese Feststellung wird durch die Ergebnisse zahlreicher Untersuchungen über den Einfluss des pH-Wertes und der Starterkulturen solide untermauert (GoEPFert und Chung 1970, Leistner u.a. 1973 b, Smith u.a. 1975 a und b, GilLILAND und SPECK 1975, SIRVIÖ u.a. 1977).

\section{Zusammenfassung und Schlussfolgerungen}

In der vorliegenden Arbeit wurde untersucht, welche Auswirkungen eine Verringerung der Nitrit- und Nitratzusätze auf die Reifung und Eigenschaften der Rohwurst hat. Der Arbeit lag die Aufgabe zugrunde, Empfehlungen über die optimale Dosierung dieser Zusatzstoffe unter Berücksichtigung der Forderung auszuarbeiten, dass sich daraus kein durch pathogene Bakterien bedingtes erhöhtes gesundheitliches Risiko und keine technologischen Schwierigkeiten ergeben dürfen.

Die Untersuchung gliedert sich in drei Teile. In den Präliminarversuchen wurden der Einfluss des Nitrit- und/oder Nitratzusatzes auf die Eigenschaften der Rohwurst untersucht; auf Grund der Ergebnisse wurden dann die Herstellungsvorschriften gewählt. Die mit verringertem Nitrit- und/oder Nitratzusatz 
hergestellten Würste wurden in technologischer Hinsicht (vergleichende Versuche) und in bezug auf das Salmonellenwachstum (Salmonellenversuche) an Würsten mit den üblichen Zusätzen verglichen. Insgesamt umfasste die Untersuchung 208 Versuchswurstchargen.

Die Präliminarversuche umfassten 16 Doppelversuchsreihen und dienten dazu, die Auswirkungen verschiedener Nitrit- und Nitratzusätze auf die Eigenschaften der Rohwurst unter verschiedenen Verhältnissen klarzustellen. Als Starterkultur für die Würste wurden entweder Micrococcus sp. (Baktoferment 61) oder Lactobacillus plantarum + Micrococcus sp. (Duploferment 66) verwendet.

1. Das zugesetzte oder aus Nitrat reduzierte Nitrit hemmte deutlich das Wachstum der Laktobazillen, nicht aber das Wachstum der Mikrokokken. Eine Verringerung des Nitritzusatzes von $100 \mathrm{ppm}$ auf $50 \mathrm{ppm}$ und des Nitratzusatzes von $125 \mathrm{ppm}$ auf $62,5 \mathrm{ppm}$ oder darunter hatte ein zu kräftiges Bakterienwachstum und einen zu starken $\mathrm{pH}$-Abfall zur Folge. Die niedrigsten Konzentrationen, die in den Versuchen geprüft wurden - $25 \mathrm{ppm} \mathrm{NaNO}_{2}$ und $50 \mathrm{ppm} \mathrm{KNO}_{3}$ - ergaben noch Wurst von beständiger, gleichmässiger Farbe. Durch Zugabe von Askorbinverbindungen konnte die Umrötung beschleunigt werden.

2. Bei Senkung der Fermentationstemperatur von $26^{\circ} \mathrm{C}$ auf $18^{\circ} \mathrm{C}$ verlangsamte sich die Nitratreduktion. Gleichzeitig nahm die Maximalkonzentration des durch Reduktion aus Nitrat gebildeten Nitıits ab. Der End-pH blieb mit abnehmender Reifetemperatur höher. Zwischen bei gleicher Temperatur hergestellten Nitrit- und Nitrat-Würsten wurden keine Unterschiede beobachtet.

Die vergleichenden Versuchsreihen wurden sechsmal, die Salmonellen-Versuchsreihen zweimal durchgeführt.

3. Bei gleichzeitigem Zusatz von Glukono-delta-Lakton (GdL) hatte eine Verringerung des Nitritzusatzes von $200 \mathrm{ppm}$ auf $100 \mathrm{ppm}$ oder beim Arbeiten mit Starterkulturen (Duploferment 66) eine Verringerung des Nitratzusatzes von $300 \mathrm{ppm}$ auf $150 \mathrm{ppm}$ oder dessen Substitution durch $50 \mathrm{ppm} \mathrm{NaNO}_{2}$ und 75 ppm $\mathrm{KNO}_{3}$ keine statistisch signifikante Verschlechterung des Geruchs, des Geschmacks oder der Schnittflächenfarbe zur Folge; allerdings lieferte der niedrigere Nitritzusatz bei GdLWürsten eine schwächere Wurstoberflächenfarbe als höherer Zusatz.

4. Die Verringerung des Nitrit- oder Nitratzusatzes oder das Arbeiten mit einem Nitrit-Nitrat-Gemisch hatte keinen Einfluss auf die physikalischen und mikrobiologischen Eigenschaften der Würste oder auf das Wachstum von Salmonella senftenberg, die bei den GdL-Würsten zahlenmässig auf null oder nahezu null, bei den Starterkultur-Würsten auf ein Zehntel der ursprünglichen Zahl zurückging.

5. Der Restnitritgehalt bewegte sich bei den GdL- vnd den StarterkulturWürsten in der gleichen Grössenordnung (10-15 ppm $\left.\mathrm{NaNO}_{2}\right)$, wohingegen der Gehalt an Restnitrat der GdL-Würste drei- bis fünfmal (ungefähr $100 \mathrm{ppm} \mathrm{KNO}_{3}$ ) so hoch wie bei den Starterkultur-Würsten $\left(20-28\right.$ ppm $\left.\mathrm{KNO}_{3}\right)$ lag. 
6. Der gegenseitige Vergleich von GdL-Würsten und Starterkultur-Würsten ergab, dass der $\mathrm{pH}$ bei ersteren schneller abnahm, jedoch auf einem höheren Wert zum Stillstand kam.

Als Folge dieser Erscheinung hatten die Starterkultur-Würste die bessere Konsistenz und Farbbeständigkeit; ausserdem waren sie den GdL-Würsten geschmacklich überlegen.

7. In sämtlichen Versuchen kam in sehr augenfälliger Weise der Nitritschwund mit Erreichen von pH 5,0 zum Stillstand. Die Ursache dieser Erscheinung wurde diskutiert. Bei der Nitratreduktion lag der ${ }^{2}$ Wendepunkt im $\mathrm{pH}$-Bereich von $5,2-5,3$, trat aber nicht in so ausgeprägter Form in Erscheinung wie beim Nitritschwund.

Schlussfolgerungen :

Auf Grund der vorliegenden Untersuchung und der damit verbundenen Literaturauswertung wurde festgestellt, dass bei Zusatz von $100 \mathrm{ppm} \mathrm{NaNO}_{2}$ oder $150 \mathrm{ppm} \mathrm{KNO}_{3}$ oder einer Mischung aus 50 ppm $\mathrm{NaNO}_{2}$ und $75 \mathrm{ppm}$ $\mathrm{KNO}_{3}$ eine technologisch und gesundheitlich sichere Rohwurstherstellung gewährleistet ist.

Voraussetzung für die Verwendung der verringerten Nitrit- oder Nitratzusätze ist allerdings, dass dabei gleichzeitig GdL oder Starterkulturen zum Einsatz kommen. Dadurch wird ein genügend rascher $\mathrm{pH}-\mathrm{Abfall}$ in den gewünschten $\mathrm{pH}$-Bereich $(\mathrm{pH} \mathrm{5,3-4,8)}$ erzielt. 


\section{LITERATUR}

Акаsні, A. 1974 a. Examination of the effective strains for the curing process of meat products III. Nitrate and nitrite reduction of the strains isolated from pickles. From English summary. Japanese J. Dairy Sci. 23: A57-A64. (FST Abstr. 1 S 128, 1974).

- -1974 b. Examining of the effective strains for the curing process of the meat products. IV. On the estimation and determination of $\mathrm{NH}_{3}$ and $\mathrm{NO}$ produced from pickle by the isolated strains. Japanese J. Dairy Sci. 23: A159-A164.

Ando, N., Nagata, Y. \& Oкоуама, T. 1971. Effects of low molecular fraction of sarcoplasma from porcine skeletal muscle on the behaviour of nitrite and the formation of cooced cured meat color in rapid curing process. Proc. 17th Eur. Meet. Meat Res. Workers, C 8. Bristol.

Anon. 1974. Ref: Proc. ISNMP 1. 1974, p. 102.

Bacus, J. N. \& Deibel, R. H. 1972. Nitrite burn in fermented sausage. Appl. Microbiol. 24: 405-408.

Barber, L. E. \& Deibel, R. H. 1972. Effect of $\mathrm{pH}$ and oxygen tension on staphylococcal growth and enterotoxin formation in fermented sausage. Appl. Microbiol. 24: 891-898.

Bergey's Manual, 1974. Bergey's Manual of Determinative Bacteriology. 1246 p. 8 th Ed. The Williams \& Wilkins Co, Baltimore.

BInkert, E. F. \& Kolari, O. 1975. The history and use of nitrate and nitrite in the curing of meat. Fd. Cosmet. Toxicol. 13:655-661.

Bodwell, C. E. \& Mclain, P. E. 1971. Proteins. In Price, J. F. \& Schweigert, B. S. (ed.): The Science of Meat and Meat Products. 2nd Ed. W. H. Freeman Co., San Francisco, p. 93.

BraAthen, O. S. 1963. Experiences with frozen meat for dry sausage production especially with regard to the colour formation. Proc. 9th Eur. Meet. Meat Res. Workers. Budapest.

Brooks, J. 1937. The action of nitrite on haemoglobin in the absence of oxygen. Proc. of the Royal Soc. B, Vol. 123: $368-382$.

--1938 . Color of Meat. Fd. Research 3: 75-78.

Buchanan, R. L. \& Sohlberg, M. 1972. Interaction of sodium nitrite, oxygen and pH on growth of $S$, aureus. J. Fd Sci. 37: 81-85.

Cassens, R. G., Woolford, G., Lee, S. H. \& Goutefongea, R. 1977. Fate of nitrite. Proc. ISNMP 2. 1977.

Castellani, A. \& Niven, C. Jr. 1955. Factors affecting the bacteriostatic action of sodium nitrite. Appl. Microbiol. 3: 154-159.

Christiansen, L. N., Tompkin, R. B., Shaparis, A. B., Johnston, R. W. \& Kaotter, D. A. 1975. Effect of sodium nitrite and nitrate on Clostridium botulinum growth and toxin production in a summer style sausage. J. Fd Sci. 40: 488-495.

Chung, K. C. \& Goepfert, J. M. 1970. Growth of salmonella at low pH. J. Fd Sci. 35: 326328.

Collins-Thompson, D. L., Sen, N. P., Aris, B. \& Schwinghamer, L. 1972. Non-enzymic in vitro formation of nitrosamine by bacteria isolated from meat products. Can. J. Microbiol. 18: 1968-1971.

Coretri, K. 1975. Rohwurst und Rohwurstwaren. 1. Teil. Rohwurst. 1. Schnittfeste Rohwurst. Reifung und Trocknung der Rohwurst. Fleischwirtschaft 55: 296-306.

- - \& Tändler, K. 1965. Einfluss der Zuckerzugabe auf die Qualität von Rohwurst. Fleischwirtschaft 45: 1055-1058. 
Crosby, N. T., Foreman, J. K., Palframan, J. F. \& Sawyer, R. 1972. Estimation of steamvolatile $\mathrm{N}$-nitrosamines in foods at the $\mu \mathrm{g} / \mathrm{kg}$ level. Nature $238: 342-343$.

- - \& SAWYER, R. 1976. N-nitrosamines: A review of chemical and biological properties and their estimation in foodstuffs. In Chichester, C. O., Mrak, E. M. \& Steward, G. F. (ed.): Adv. Fd. Research, Vol. 22:1-71.

Daly, C., Lachance, M. la, Sandine, W. E. \& Elliker, P. R. 1973. Control of S. aureus in sausage by starter culture and chemical acidulation. J. Fd Sci. 38: 426-430.

Deibel, R. H. 1974. Technology of fermented, semi-dried and dried sausages. Proc. Meat Ind. Res. Conf. pp. 57-60. AMIF, Arlington, Virginia.

Dethmers, A. E., Rock, H., Fazio, T. \& Johnston, R. W. 1975. Effect of added sodium nitrite and sodium nitrate on sensory quality and nitrosamine formation in thüringer sausage. J. Fd Sci. 40: 491-495.

Dilova, N., Nestorov, N., Grozdanov, A. \& Dikova, G. 1976. Changes in the contents of sulfhydryl groups and protein-bound nitrites in raw dried sausages. Proc. 22nd Eur. Meet. Meat Res. Workers. G 1. 1976. Malmő.

EмоDi, A. S. \& Leсношісн, R. V. 1969. Low temperature growth of type E Clostridium botulinum spores. 1. Effects of sodium cloride, sodium nitrite and $\mathrm{pH}$. J. Fd Sci. 34: 78-81.

FAO/WHO 1974. Toxicological evaluation of some food additives including anticaking agents, antimicrobials, antioxidants, emulsifiers and thickening agents. WHO Fd Additives Ser. 5: 97-109 (Nitrite), 92-96 (Nitrate).

FAzIO, T., White, R. H. \& HowARD, J. W. 1971. Analysis of nitrite- and/or nitrate- processed meats for N-nitrosodimethylamine. J. AOAC 54: 1157-1159.

Fox, J. B., Jr. 1966. The chemistry of meat pigments. Fd Processing 14: 207-210.

- - \& Ackerman, S. A. 1968. Formation of nitric oxide myoglobin: Mechanisms of the reaction with various reductants. J. Fd Sci. 33:364-370.

- - \& Nicholas, R. A. 1974. Nitrite in meat. Effect of various compounds on loss of nitrite. J. Agric. Fd Chem. 22: 302-306.

- - \& Thомson, J. S. 1963. Formation of bovine nitrosylmyoglobin. Biochemistry 2: $465-$ 470 .

- - \& Tномsоn, J. S., 1964. The formation of green heme pigments from metmyoglobin by the action of nitrite. Biochemistry 3:1323-1328.

Genigeorgis, C., Martin, S., Franti, C. E. \& Riemann, H. 1971. Initiation of staphylococcal growth in laboratory media. Appl. Microbiol. 21: 934-939.

Gerhard, V. \& Haller, S. 1973. Nitrat- und Nitritgehaltsbestimmungen in GdL-haltigen Rohwürsten. Fleischwirtschaft 53: 548-554.

GiLliland, S. E. \& SPECK, M. L. 1972. Interactions of food starter cultures and food-borne pathogens: lactic streptococci versus staphylococci and salmonellae. J. Milk Fd Techn. 35: $307-310$.

Goepfert, J. M. \& ChUng, K. G. 1970. Behaviour of Salmonella during the manufacture and storage of a fermented sausage product. J. Milk Fd Technol. 33: 185-191.

Grau, R. \& Mirna, A. 1957. Z. Analyt. Chem. 158: 182. Ref. STOYA 1969.

Grever, A. B. \& Schuddenboom, L. J. 1958. Isolierung von bei Fleischwaren Grünärbung verursachenden Milchsäurebakterien. Fleischwirtschaft 10: 234-235.

GöTSE, V. 1969. Bestimmung von Myoglobin und Hämoglobin im Fleischextrakt von Schlachttieren. Fleischwirtschaft 49: 901-906.

HAmm, R. \& Schweiger, A. 1964. Über das Farbhaltungsvermögen von Rindfleisch. I. Einfluss von Kationen. Fleischwirtschaft 44: 773-778.

Hill, L. H., Webв, W. B., Moncol, N. D. \& Adams, A. T. 1973. Changes im residual nitrite in sausage and luncheon meat products during storage. J. Milk Fd Technol. 36: 515-519.

HıLl, W. M. 1973. Persönliche Mitteilung. Ref. TAtinı u. a. 1973.

Hornsey, H. C. 1956. The colour of cooced cured pork I. Estimation of the nitric oxide haem pigments. J. Sci. Fd Agric. 7: 534-540.

INGRAM, M. 1974. The microbial effects of nitrite. Proc. ISNMP 1. 1974, pp. 63-75.

Jedlicka, G. J., Wilcox, J. C., McCall, W. A. \& Gacula, M. C., Jr. 1975. Effects of varying levels of nitrate and nitrite on Staphylococcus aureus growth in pepperoni. Abstr. Ann. Meet. Amer. Soc. Microbiology, p. 200. 
Kahan, G., Cooper, D., Papavasiliou, A. \& Kramer, A. 1973. Expanded tables for determining significance of differences for ranked data. Fd Technol. 27, 5: 64-69.

Kitchell, A. G. \& Shaw, B. G. 1975. Lactic acid bacteria in fresh and cured meat. In CARR, J. G., Cutring, C. V. \& Whiting, G. C. (ed.): Lactic Acid Bacteria in Bevegares and Food. III 2: 209-220. AP, London \& New York.

Kolzumi, C. \& Brown, D. 1971. Formation of nitric oxide myoglobin by nicotin amide adenine dinucleotides and flavins. J. Fd Sci. 36: 1105-1109.

Kotter, L., Fischer, A. \& Schmidt, H. 1976. Zum Vorkommen von Nitrosaminen in Fleischerzeugnissen und Untersuchungen an schnittfesten Rohwürsten bei, unterschiedlichen Zusätzen. Fleischwirtschaft 56: 997-1007.

Kueper, T. V. \& Trelease, R. D. 1974. Variables affecting botulinum toxin development and nitrosamine formation in fermented sausages. Proc. Meat Ind. Res. Conf. 1974. pp. 6974. AMIF, Arlington, Virginia.

LABots, H. 1977. Effect of nitrite on the development of Staphylococcus aureus in fermented sausages. Proc. ISNMP 2. 1977.

LANE, R. P., RICE, R. H. \& BEILEy, M. E. 1974. Gas chromatographic-mass spectrometric determination of $\mathrm{N}$-nitrosodimethylamine formed in synthetic and human gastric juice. J. Agric. Fd Chem. 22: 1019-1023.

Lawrie, R. A. 1974. Meat Science. 2. Aufl. 419 p. Pergamon Press, Braunschweig.

Lawrowa, L. P., Solowjew, W. I., Kaljenowa, M. S., Buschkowa, L. A., Morosowa, L. I., Jeriemina, G. K., Stschjegolewa, O. P. \& RJabowa, T. I. 1967. Ùber die Möglichkeit der Herabsetzung des Gehaltes an freien Nitriten und Nitraten in den Wurstwaren. Ber. 13. Eur. Fleischforscherkongress. G 7. Rotterdam.

Lechowich, R. V., Evans, J. B. \& Nrven, C. F., Jr. 1956. Effect of curing ingredients and procedures on the survival and growth of staphylococci in and on cured meats. Appl. Microbiol. 4: $360-363$.

Leistner, L., Hechelmann, H., Bem, Z. \& Albertz, R. 1973 a. Untersuchungen zur Reduktion des Nitritzusatzes zu Fleischerzeugnissen. Fleischwirtschaft 53:1751-1754.

- - - Hechelmann, H. \& Uchida, K. 1973 b. Welche Konsequenzen hätte ein Verbot oder eine Reduzierung des Zusatzes von Nitrat oder Nitritpökelsalz zu Fleischerzeugnissen? Aus mikrobiologischer Sicht. Fleischwirtschaft 53: 371-378.

LiJinsky, W. \& EPSTEIN, S. S. 1970. Nitrosamines as environmental carcinogens. Nature 225: $21-23$.

Magee, P. N. \& Barnes, J. M. 1967. Carcinogenic nitrosocompounds. Adv. Cancer Res. 10: $163-246$.

McLean, R. A., Lilly, H. D. \& Alford, J. A. 1968. Effects of meat-curing salts and temperature on production of staphylococcal enterotoxin B. J. Bacteriol. 95: 1207-1211.

Marazza, V. \& Crespi, A. 1963. Observazioni sulla sopravvirenza di Salmonella cholerasuis in insaccati naturalmente inquinati. (Lebensdauer von Salmonella choleraesuis in natürlich infizierten Würsten.) Atti d. Soc. Ital. d. Sci. Vet. 17: 537-541.

Menster, J. 1965. The application of glucono-delta-lactone in meat products. Proc. 11. Eur. Meet. Meat Res. Workers, H-3. Beograd.

Mrrna, A. 1970. Uber die Umsetzung von Nitrit in Fleischwaren und dessen Verteilung in verschiedenen Fraktionen. Ber. 16. Eur. Fleischforscherkongress, C 16. Varna.

- 1971. Zusatzstoffe, Hilfsstoffe und Rückstände in Fleisch und Fleischwaren. Wien. tierärztl. Monatschrift 58: 396-402.

- -1973 . Welche Konsequenzen hätte ein Verbot oder eine Reduzierung des Zusatzes von Nitrat und Nitritpökelsalz zu Fleischerzeugnissen? Aus chemischer Sicht. Fleischwirtschaft 53: 357-360.

$-\rightarrow$ - \& Coretri, K. 1974. Uber den Verbleib von Nitrit in Fleischwaren. II. Untersuchungen über chemische und bakteriostatische Eigenschaften verschiedener Reaktionsprodukte des Nitrits. Fleischwirtschaft 54: 507-510.

- _ \& Hofmann, K. 1969. Über den Verbleib von Nitrit in Fleischwaren. I. Umsetzung von Nitrit mit Sulfhydryl-Verbindungen. Fleischwirtschaft 49: 1361-1366.

- - \& Sснӥтz, G. (1970). Zur Nitritbestimmung in Anwesenheit von Ascorbinsäure. Bundesanst. f. Fleischforsch., BRD, Jahresbericht s. G $83-84$. 
— - \& Schürz, G. 1972. Verfahren zur gleichzeitigen Bestimmung des Pökelfarbstoffes sowie von Nitrit und Nitrat in Fleischerzeugnissen. Fleischwirtschaft 52: 1337-1338.

Mirvish, S. S. 1975. Formation of N-nitrosocompounds: chemistry, kinetics, and in vivo occurrence. Toxicol. and Appl. Pharmacol. 31: 325-351.

MöHLer, K. 1958. Zur Bestimmung des Pökelfarbstoffes. Z. Lebensm.- Unters. u. -Forsch. 108: $20-28$,

— - 1959. Ascorbinsäure als Zusatz zu Fleischerzeugnissen II. - I. Chemische und technologische Probleme. Deutsche Lebensm.- Rundschau 55:3-6.

_, 1970. Bilanz der Bildung des Pökelfarbstoffs im Muskelfleisch. I. Mitt.: Oxydation von Muskelpigment und Nitrit. Z. Lebensm.- Unters. u. -Forsch. 142: 169-179.

- -1974 . Formation of curing pigments by chemical, biochemical or enzymatic reactions. Proc. ISNMP 1. 1974. pp. 13-19.

- - \& Hallermayer, E. 1974. Nitrosamine in Lebensmitteln. Beiträge zu Analytik, Abbau und Aufbau. I. Bestimmungsmethode für Modellversuche. Z. Lebensm.- Unters, u. -Forsch. 156: $206-210$.

Nagata, Y. \& Mirna, A. 1974. Einfluss der Verarbeitung auf die Bildung von Nitrosaminen in Fleischwaren. Fleischwirtschaft 54:1781-1789.

NCDC 1971 a. National Center for Disease Control. Staphylococcal gastroenteridis associated with salami. United States Morbidity Mortality 20: 253. Ref.: DALy et al. 1973.

- 1971 b. Staphylococcal gastroenteridis associated with salami. United States Morbidity Mortality 20: 264. Ref. Daly et al. 1973.

Nestorov, N., Dilova, N., Grozdanov, A. L., Djevisov, S. \& Kiseva, R. 1975. Formation and stability of nitrosopigments in raw-dried sausages depending on the composition of the salting mixtures. Proc. 21st Eur. Meet. Meat Res. Workers, pp. 203-205. Bern.

NinntvaARA, F. P. 1955. Uber den Einfluss von Bakterienreinkulturen auf die Reifung und Umrötung der Rohwurst. Acta Agr. Fenn. 84. 128 S. Helsinki.

— - \& Antila, P. 1972. Der Nährwert des Fleisches. Fleischforschung und Praxis 8. 181 S. Verlag der Rheinhessischen Druckwerkstätte, Alzys.

- - \& РонјА, M. S. 1957. Erfahrungen über die Herstellung von Rohwurst mittels einer Bakterienreinkultur. Fleischwirtschaft 9: 789-790.

Niskanen, A. \& Nurmi, E. 1976. Effects of starter culture on staphylococcal enterotoxin and thermonuclease production in dry sausage. Appl. Environm. Microbiol. 31: 11-20.

Nordin, H. R. 1969. The depletion of added sodium nitrite in ham. Can. Inst. Fd Technol. J. 2: $79-85$.

Nurmi, E. 1966 a. Effect of Bacterial Inoculations on Characteristics and Microbial Flora of Dry Sausage. Acta Agr. Fenn. 108. 77 p. Helsinki.

$-1966 \mathrm{~b}$. Studies on the acceleration of the ripening process of dry sausage. Proc. 12th Eur. Meet. Meat Res. Workers, E 8. Sandefjord.

- - , Raevuori, M. \& Hill, P. 1975. The effect of nitrite and certain other food additives on the quality of Finnish cooced sausage. Proc. 21st Eur. Meet. Meat Res. Workers. pp. 209-211. Bern.

- - \& TuRUnen, S. O. 1970. The effect of nitrite on the growth curve of lactobacilli and micrococci. Proc. 16th Eur. Meet. Meat Res. Workers, B 5. Varna.

Orsman, W. J. 1977 a. Chemical behaviour of nitrite in meat products. I. The stability of protein bound nitrite during storage. Proc. ISNMP 2. 1977.

- 1976 b. Chemical behaviour of nitrite in meat products. II. Effect of iron and EDTA on the stability of protein-bound nitrite. Proc. ISNMP 2. 1977.

- - \& Krol, B. 1972. Depletion of nitrite in heated meat products during storage. Proc. 18th Eur. Meet. Meat Res. Workers. Vol. II: 409-415. Guelph.

Ortmann, S. 1967. Ein Beitrag zur Überlebenszeit von Salmonellen in Rohwursten. Monatsheft. Vet. Med. 22: 422-424.

ÖSTLUND, K. \& REGNER, B. 1968. Undersökningar rörande mikrofloran i isterband. (Untersuchungen über die Mikroflora der schwedischen fermentierten Fleischwaren "Isterband».) Nord. Vet.-Med. 20: 527-542.

Palumbo, S. A., Smith, J. L., Centilore, K. M. \& Fiddler, W. 1974. Investigations on the possible occurrence of nitrosamines in lebanon bologna. J. Fd Sci. 39: 1257-1258. 
Panalaks, T., Iyengar, J. R. \& Sen, N. P. 1973. Nitrate, nitrite, and dimethylnitrosamine in cured meat products. J. AOAC 56: 621-625.

- - , Donaldson, B. A., Mrles, W. F. 1974. Further survey of cured meat products for volatile N-nitrosamines. J. AOAC 57; 806-812.

$\operatorname{PETÄJA}$, E. 1977. The effect of some gram negative bacteria on the ripening and quality of dry sausage. Doctoral thesis. Department of Meat Technology, University of Helsinki. (In preparation).

PfeIL, E. \& LIEPE, H.-U. 1973 a. Gewinnung von Fermenten aus Rohwurst-Reifungsbakterien? Fleischwirtschaft 53:221-222.

_- - \& LiePE, H.-U. 1973 b. Der Einfluss des Nitratreduktasesystems auf den Restnitritgehalt in Rohwurst und seine Abhängigkeit von ảusseren Bedingungen. Fleischwirtschaft 53: $1745-1748$.

- - \& Liepe, H.-U. 1974. Nitrat, Nitrit und Bakterien bei der Herstellung von Rohpökelwaren. Fleischwirtschaft 54: 1717-1718.

Рон ј A, M. S. 1960. Micrococci in Fermented Meat Products. Acta Agr. Fenn. 96.80 p. Helsinki.

$\rightarrow-\&$ KnUtar, U. 1975. Unpublizierte Information.

- - \& NinnvaARA, F. P. 1957. Über die Reifung der Rohwurst. III. Mitteilung. Über die antagonistische Wirkung eines Mikrokokkenstammes gegen die in Rohwurst vorkommenden Bakterienstämme. Z. Lebensm.- Unters. u. -Forsch 106: 298-301.

Proc. ISNMP 1. 1974. Proceedings of International Symposium on Nitrite in Meat Products. 268 p. Pudoc, Wageningen.

Proc. ISNMP 2. 1977. Proc. of the Second International Symposium on Nitrite in Meat Products, Zeist, Holland. (Im print).

Puolanne, E., Törmä, P. \& Djedjeva, G. 1977. Über das Nitratreduktionsvermögen der Stämme Micrococcus M III und Vibrio 21. Lebensm.- Wiss.-Techn. (Im Druck).

RAEVUORI, H. 1975. Effect of nitrite and erythorbate on growth of Bacillus cereus in cooced sausage and in laboratory media. Zbl. Bakt. Hyg., I. Abt. Orig. B 161: 280-287.

$\ldots-1976$. Studies of Bacillus cereus as a food-poisoning organismn with special reference to the effects of certain preservatives on growth of the bacterium. Doctoral thesis, The College of Veterinary Medicine, Helsinki, Finland. 79 p.

- - \& Genigeorgis, C. 1975. Effect of $\mathrm{pH}$ and sodium cloride on growth of Bacillus cereus in laboratory media and certain foods. Appl. Microbiol. 29: 68-73.

Regier, L. W. \& TAppel, A. L. 1956. Freeze-dried meat. III. Non-oxidative deterioration of freeze dried beef. Fd Res. 21: 630-639.

Reith, J. F. \& Szakńly, M. 1967 a. Formation and stability of nitric oxide myoglobin. I. Studies with model systems. J. Fd Sci. 32: 188-193.

- - \& Szakály, M. 1967 b. Formation and stability of nitric oxide myoglobin. II. Studies on meat. J. Fd Sci. 32: 194-196.

ReUter, G. 1972. Versuche zur Rohwurstreifung mit Laktobazillen- und Mikrokokken-Starterkulturen. Fleischwirtschaft: $465-473$.

Riemann, H., Lee, W. H. \& Genigeorgis, C. 1972. Control of Clostridium botulinum and Staphylococcus aureus in semi-preserved meat products. J. Milk Fd Technol. 35: 514523.

Roberts, T. A. \& Ingram, M. 1977. The significance of nitrite and nitrate in the control of Clostridium botulinum in cured meats. Proc. ISNMP 2. 1977.

RöDEL, W. 1973. Messung der Wasseraktivität unter Praxisbedingungen. Fleischwirtschaft 53: $27-31$.

- - , Ponert, H. \& Leistner, L. 1975. Einstufung von Fleischerzeugnissen in leicht verderbliche, verderbliche und lagerfähige Produkte. Ber. 21. Eur. Fleischforscherkongress, S. 77-78. Bern.

Rozier, J. 1971. Die Rolle der Katalase-Aktivität des Fleisches bei der Rohwurst-Fabrikation. Fleischwirtschaft 51: 1063-1066.

SAIR, L. 1963. A, B and C of meat curing. Nat. Provisioner 148: 18-21.

Sander, J. 1968. Nitrosaminsynthese durch Bakterien. Hoppe-Seyler's Z. physiolog. Chem. 349: 429-432. 
- - , Schweisberg, F. 1972. In vivo and in vitro experiments on the formation of $\mathrm{N}$-nitrosocompounds from amines or amides and nitrate or nitrite. Proc. IARC Scientific Publ 3: $97-103$.

- - , Schweisberg, F. \& Menz, H. P. 1968. Untersuchungen über die Entstehung cancerogener Nitrosamine im Magen. Hoppe Seyler's Z. physiol. Chem. 349: 1691-1697.

_ - \& SEIF, F. 1969. Bakterielle Reduktion von Nitrat im Magen des Menschen als Ursache einer Nitrosaminbildung. Arzneimittel-Forsch. 19: 1091-1093.

SCHEIBNER, G. 1969. Beobachtungen über das Verhalten von Salmonellen beim Räuchern von Rohwurst. Monatsheft Vet.-Med. 24:388-391.

Schormüller, J. \& Schilling, M. 1961. Über die Nitratreduktase einiger in der Rohwurst vorkommender Mikroorganismen. Die Nahrung 5: 18-40.

- $-\&$ Schillın'; M. 1963. Weitere Untersuchungen über die Nitratreduktase verschiedener an der Reifung von Rohwurst beteiligter Mikroorganismen. Z. Lebensm.- Unters, u. -Forsch. 118: $492-508$.

Scotr, W. J. 1957. Water relations of food spoilage microorganisms. Adv. Fd Res. 7: 83-127.

SEN, N. P. 1972. The evidence for the presence of dimethylnitrosamine in meat products. Fd Cosmet. Toxicol. 10: 219-223.

Sirviö, P., Nurmi, E., Puolanne, E. \& Ninnivaara, F. P. 1977. Der Einfluss von Starterkulturen und einigen Zusatzstoffen auf das Wachstum von Salmonella senftenberg. Fleischwirtschaft (Im Druck).

SkJelkvỉle, R., TJaberg, T. B. \& Walland, M. 1974. Comparison of salami sausage produced with and without addition of sodium nitrite and sodium nitrate. J. Fd Sci. 39: 520-524.

Smith, J. L., Huhtanen, C. N., Kissinger, J. C. \& Palumbo, S. A. 1975 a. Survival of Salmonellae during pepperoni manufacture. Appl. Microbiol. 30: 759-763.

- - , Palumbo, S. A., Kissinger, J. C. \& Huhtanen, C. N. 1975 b. Survival of Salmonella dublin and Salmonella typhimurium in Lebanon Bologna. J. Milk Fd. Techn. 38: 150 - 154.

Snyder, H. E. \& Ayres, J. C. 1961. The autoxidation of crystallized beef myoglobin. J. Fd Sci. 26: $469-474$.

StoyA, W. 1969. Vereinfachte Nitratbestimmung in Lebensmitteln, insbesondere in Fleischwaren. Deutsche Lebensm.- Rundschau 65: 114-148.

TAKacs, J. \& Srmonffy, Z. 1970. Das Salmonellaproblem bei Dauerwürsten. Fleischwirtschaft 50: 1200-1202.

Tannenbaum, S. R. 1976. Relative risk of nitrate and nitrite ingestion. Proc. Meat. Ind. Res. Conf. pp. 25-33. AMIF, Arlington.

- - , Sinskey, A. J., Weisman, M. \& Bishop, W. 1974. Nitrite in human saliva. Its possible relationship to nitrosamine formation. J. Nat. Cancer Inst. 53: 79-84.

TARLAGDIS, B. G. 1962. Interpretation of the spectra of meat pigments. II. Cured meats. The mechanism of colour fading. J. Sci. Fd Agric. 13: 485-491.

TARnay, I. 1975. Salmonellák és Staphylococcus aureus ismétlödö elöfordulása nyers füstöltkoldászmintákdan. [Salmonellen und Staphylococcus aureus werden oft in der Rohwurst gefunden.] Maguar Allatorvosok Lapja 30: 730-731.

TAtini, S. R., Lee, R. Y., McCall, W. A. \& Hill, W. M. 1976. Growth of Staphylococcus aureus and production of enterotoxins in pepperoni. J. Fd Sci. 41: 223-225.

TJaberg, T. B., Haugam, M. \& Nurmi, E. 1969. Studies on discoloration of Norwegian salami sausage. Proc. 15th Eur. Meet. Meat Res. Workers. B 5. Helsinki.

Troller, J. A. \& Stinson, J. V. 1975. Influence of water activity on growth and enterotoxin formation by $S$. aureus in food. J. Fd Sci. 40: 802-804.

TändLER, K. 1963. Die Verwendung von Zuckerstoffen bei der Rohwurstherstellung. Fleischwirtschaf $43: 804-811$.

VAndekerckhove, P. \& Demeyer, D. 1976. Amines in dry fermented sausage. Proc. 22nd Eur. Meet. Meat Res. Workers. G 2. Malmö.

Walsh, K. A. \& Rose, D. 1956. Factors affecting the oxidation of nitric oxide myoglobin. Agric. Fd Chem. 4: 352-355.

Walters, C. L., Burger, I. H., Jewell, G. G. \& Lewis, D. F. 1974. Mitochondrial enzyme pathways and their possible role during curing. Z. Lebensm.- Unters. u. -Forsch. 158: $193-203$. 
- - , TAYlor, A. McM., Casselden, R. J. \& RAY, N. 1968. Investigation of specific reducing systems in relation to meat curing. Res. Rep. 139, British Fd Manuf. 3nd. Res. Ass., Leatherhead. Ref. Möhler (1974).

WirTh, F. 1973. Welche Konsequenzen hätte ein Verbot oder eine Reduzierung des Zusatzes von Nitrat oder Nitritpökelsalz. Aus technologiscker Sicht. Fleischwirtschaft 53: $363-368$.

,--1974 . Technologie der Nitrat-/Nitritminderung bei Rohwurst. Kulmbacher Woche 1974. Kurzfassungen der Fachvorträge, 535-36.

Woolford, G., Cassens, R. G., Greaser, M. L. \& Sebranek, J. G. 1976. The fate of nitrite: reaction with protein. J. Fd Sci. 41: 585-588.

ZAtó́ıL, O. \& BRÜN, C. Sc. 1963. Zur Frage der Nitritwirkung in Fleischwaren. Fleischwirtschaft 43: 798-803.

Zyss, E. F., Perowa, P. W. \& LongrnowA, M. M. 1967. Zur Frage des Überlebens, der Entwicklung und Toxinbildung von $\mathrm{Cl}$. perfringens in Rohwurst während deren Herstellung und Lagerung. Ber. 13. Eur. Fleischforscherkongress. B 10. Rotterdam. 


\title{
Alennettujen nitriitti- ja nitraattilisäyksien vaikutus kestomakkaran ominai- suuksiin.
}

\author{
Eero Puolanne \\ Helsingin Yliopisto, lihateknologian laitos
}

Tässä työssä tutkittiin, mitä nitriitti- ja nitraattilisäyksen alentaminen vaikuttaa kestomakkaran kypsymiseen ja ominaisuuksiin. Tämän työn tarkoituksena oli laatia sellaiset käyttömääräsuositukset näille lisäaineille, ettei niin menetellen patogeenisten bakteerien aiheuttama terveydellinen riski kasva ja ettei esiinny teknologisia vaikeuksia.

Tutkimus jakautui kolmeen osaan. Alustavissa kokeissa tutkittiin nitriitti- ja/tai nitraattilisäyksien vaikutusta kestomakkaran ominaisuuksiin. Näiden tulosten perusteella valittiin valmistusohjeet tutkimuksen muihin osiin. Alennettujen nitriitti- ja/tai nitraattilisäyksien avulla valmistettuja makkaroita verrattiin yleisesti käytössä olevien lisäysten mukaisesti valmistettuihin makkaroihin teknologisesti (vertailukokeet) ja salmonellan kasvun suhteen(salmonellakokeet). Tutkimusta varten valmistettiin yhteensä 208 makkarakoe-erää.

Alustavissa kokeıssa oli 16 koesarjaa, ja kukin koesarja tehtiin kaksi kertaa. Alustavien kokeiden tarkoituksena oli tutkia eri nitriitti- ja nitraattilisäyksien vaikutuksia kestomakkaran ominaisuuksiin eri olosuhteissa. Makkaroissa käytettiin starterkulttuureina joko Micrococcus sp.:ta (Baktoferment 61) tai Lactobacillus plantarum + Micrococcus sp:ta (Duploferment 66).

1. Lisätty tai nitraatista pelkistynyt nitriitti esti selvästi laktobasillien mutta ei mikrokokkien kasvua. Nitriittilisäyksen alentaminen 100 ppm:stä 50 ppm:ään ja nitraattilisäyksen 125 ppm:stä 62,5 ppm:ään tai alemmaksi aiheutti liian voimakkaan bakteerikasvun ja $\mathrm{pH}$ arvon laskun (kuvat $6-8,10-12,16,31$ ja 32). Alhaisimmat kokeissa käytetyt lisäykset, 25 ppm $\mathrm{NaNO}_{2}$ ja 50 ppm $\mathrm{KNO}_{3}$, antoivat kuitenkin vielä makkaraan kestävän tasaisen värin. Askorbiiniyhdisteitä käyttämällä voitiin värinmuodostusta nopeuttaa.

2. Lämpötilan laskeminen $26^{\circ} \mathrm{C}$ :sta $18^{\circ} \mathrm{C}$ :een hidasti nitraatin pelkistymistä (kuva 26). Samalla jäi nitraatista pelkistynyt nitriitin maksimipitoisuus alhaisemmaksi. Loppu-pH-arvo jäi lämpötilan laskiessa korkeammaksi (kuvat 29, 30, 33 ja 34). Samassa lämpötilassa valmistettujen nitriitti- ja nitraattilisäysten avulla valmistettujen makkaroiden välillä ei ollut eroja missään tutkitussa lämpötilassa.

Vertailukokeet tehtiin kuusi kertaa ja salmonellakokeet kaksi kertaa.

3. Käytettäessä glukono-delta-laktonia (GdL) nitriittilisäyksen alentamisella 200 ppm:stä 100 ppm:ään tai starterkulttuureita käytettåessä nitraattilisäyksen alentamisella 300 ppm:stä 150 ppm:ään tai nitraatin korvaamisella 50 ppm:llä $\mathrm{NaNO}_{2}$ ja 75 ppm:llä $\mathrm{KNO}_{3}$ ei ollut tilastollisesti merkitsevää vaikutusta hajuun, makuun tai leikkauspinnan väriin (taulukot 27 ja 29). Kuitenkin alhaisemman nitrittiilisäyksen avulla valmistetussa makkarassa oli heikompi makkaran kuoren väri kuin korkeamman nitriittilisäyksen avulla valmistetussa makkarassa.

4. Nitriitti- tai nitraattilisäyksen pienentämisellä tai seoksen käytöllä ei ollut vaikutusta makkaroiden fysikaalisiin ja mikrobiologisiin ominaisuuksiin (kuva 38, taulukot 11-24) eikä Salmonella senftenbergin kasvuun, jonka lukumäärä laski GdL-makkaroissa nollaan tai lähelle nollaa ja starterkulttuurimakkaroissa kymmenenteen osaan alkuperäisestä määrästä (kuva 39, taulukko 31). 
5. GdL-makkaroiden ja starterkulttuurimakkaroiden jäännősnitriittipitoisuus oli samaa suuruusluokkaa $\left(10-15 \mathrm{ppm} \mathrm{NaNO}_{2}\right.$ ) (kuva 36, taul. 15), mutta sen sijaan jäännösnitraatin määrä oli GdL-makkaroissa kolmesta viiteen kertaa suurempi (noin $100 \mathrm{ppm} \mathrm{KNO}_{3}$ ) kuin starterkulttuurimakkaroissa $\left(20-28 \mathrm{ppm} \mathrm{KNO}_{3}\right.$, kuva 37 , taul. 16).

6. Vertailtaessa GdL-makkaroita ja starterkulttuurimakkaroita todettiin, että GdL-makkaroiden $\mathrm{pH}$-arvo laski nopeammin kuin starterkulttuurimakkaroiden, mutta loppu-pH-arvo oli kuitenkin korkeampi (taul. 22). Tämän vuoksi starterkulttuurimakkaroissa oli parempi rakenne (taul. 24) ja värin säilyvyys (taul. 28) ja lisäksi niissä oli parempi maku kuin GdLmakkaroissa (taul. 29).

7. Kaikissa kokeissa nitriitin määrän väheneminen lakkasi $\mathrm{pH}$-arvon saavutettua arvon 5,0 (kuva 40). Nitraatin pelkistymisessä tämä käännekohta oli pH-arvossa 5,2-5,3 (kuva 41), mutta se ei esiintynyt yhtä selvänä kuin nitriitin häviämisessä.

\section{Loppupäätelmät:}

Tämän tutkimuksen ja siihen liittyvän kirjallisuustutkimuksen perusteella todettiin, että teknologisesti ja terveydellisesti turvallisen kestomakkaran valmistus on mahdollista käyttämällä $100 \mathrm{ppm} \mathrm{NaNO}_{2}$ tai $150 \mathrm{ppm} \mathrm{KNO}_{3}$ tai seoksena $50 \mathrm{ppm} \mathrm{NaNO}_{2}$ ja $75 \mathrm{ppm} \mathrm{KNO}_{3}$. Edellytyksenä nitriitti- tai nitraattilisäyksen alentamiselle kuitenkin on, että samanaikaisesti käytetään GdL:ia ja/tai starterkulttuureita. Niitä käyttämällä varmistetaan tarpeeksi nopea $\mathrm{pH}$-arvon lasku toivotulle $\mathrm{pH}$-arvoalueelle $(\mathrm{pH} 5,3-4,8)$.

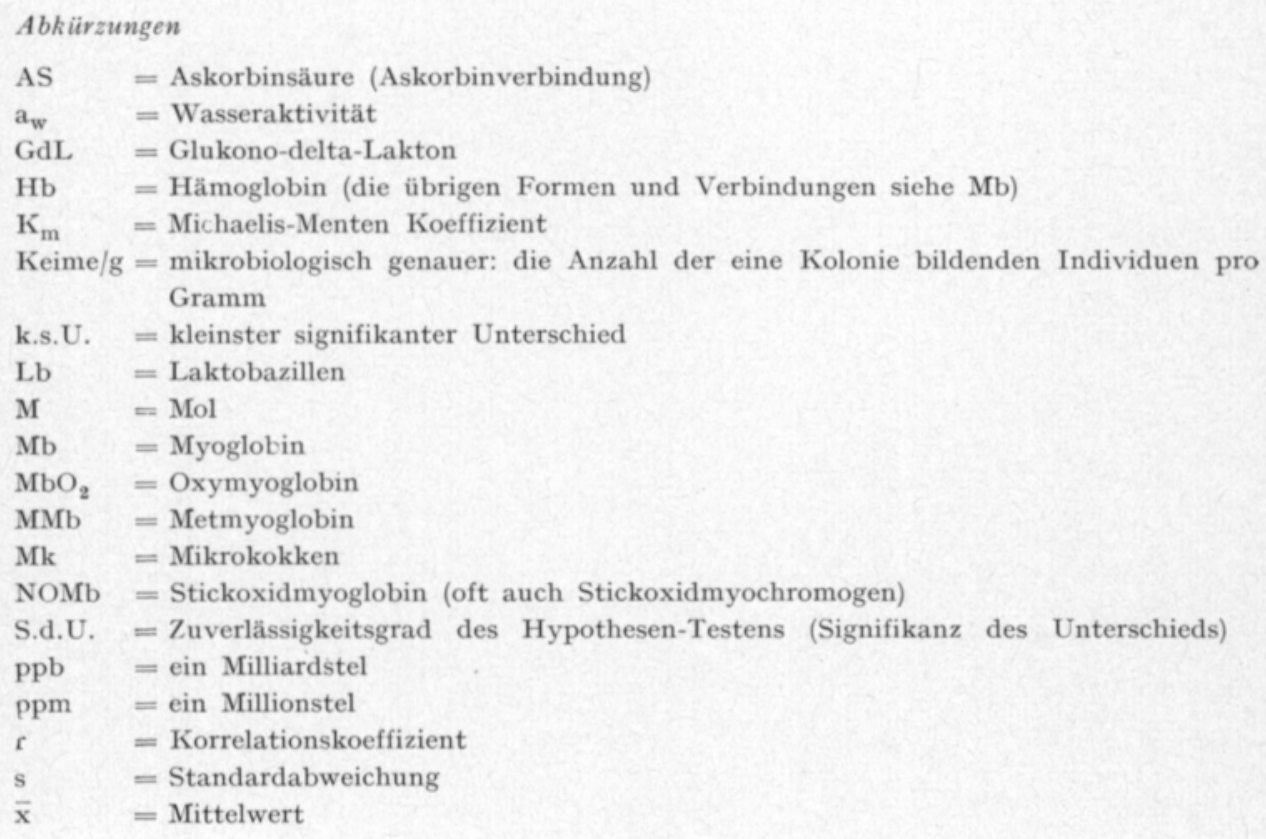



Foto 1.

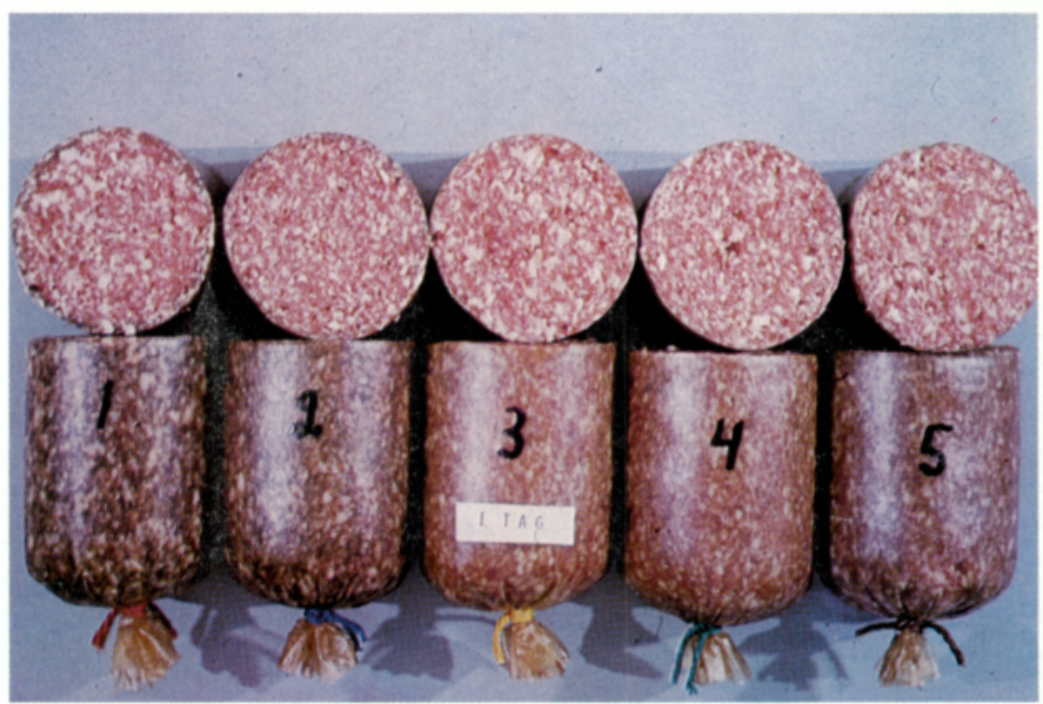

Foto 1. Die Farbe von einen Tag alten Würsten.

GdL-Würste: 1.100 ppm $\mathrm{NaNO}_{2}$

$$
\text { 2. } 200 \mathrm{ppm} \mathrm{NaNO}_{2}
$$

Starterkultur-Würste: $3.150 \mathrm{ppm} \mathrm{KNO}_{3}$

4. $300 \mathrm{ppm} \mathrm{KNO}_{3}$

5. $50 \mathrm{ppm} \mathrm{NaNO}_{2}$ und $75 \mathrm{ppm} \mathrm{KNO}_{3}$

Foto 2. Die Farbe von drei Tage alten Würsten.

Nummer: siehe Foto 1

Foto 3. Die Farbe von sieben Tage alten Würsten.

Nummer: siehe Foto 1.

Foto 4. Die Farbe von 14 Tage alten Würsten.

Nummer: siehe Foto 1.

Foto 5. Die Farbe von frischen, 1 Std. und 3 Stdn. alten Schnittflächen der 22 Tage alten Würste.

Nummer: Siehe Foto 1.

Foto 2 .

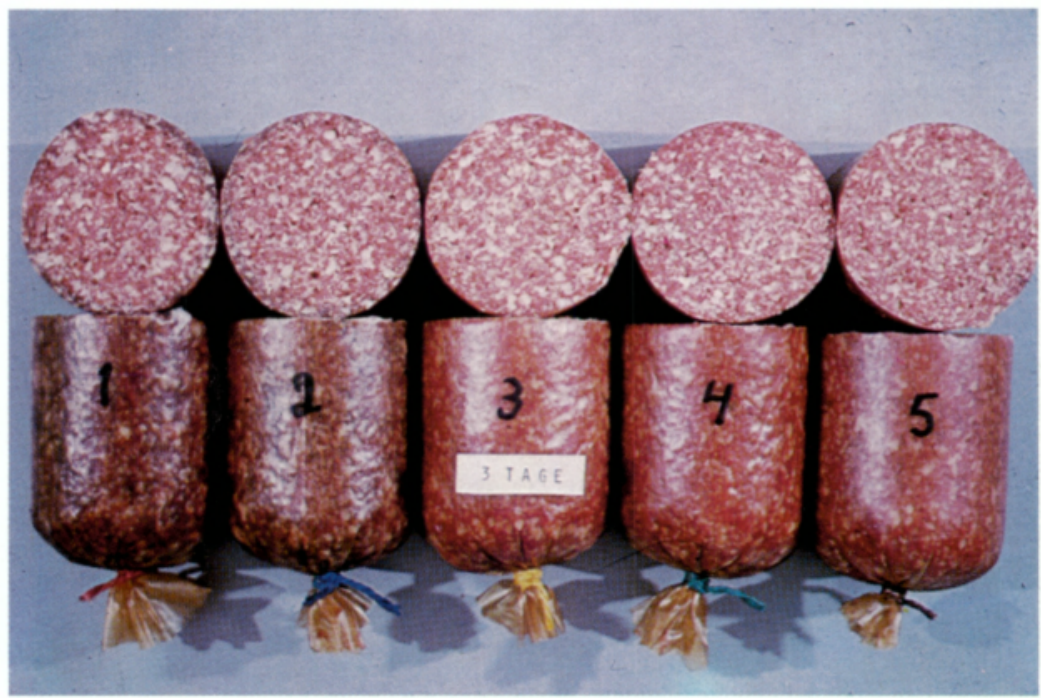




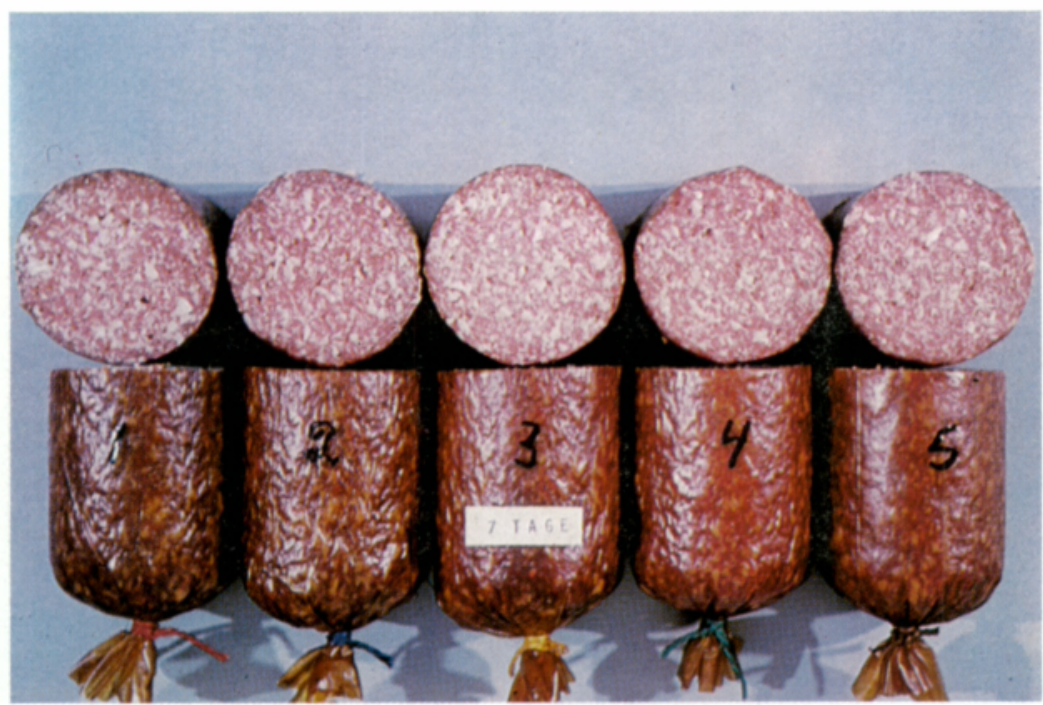

Foto 3.

Foto 4 .
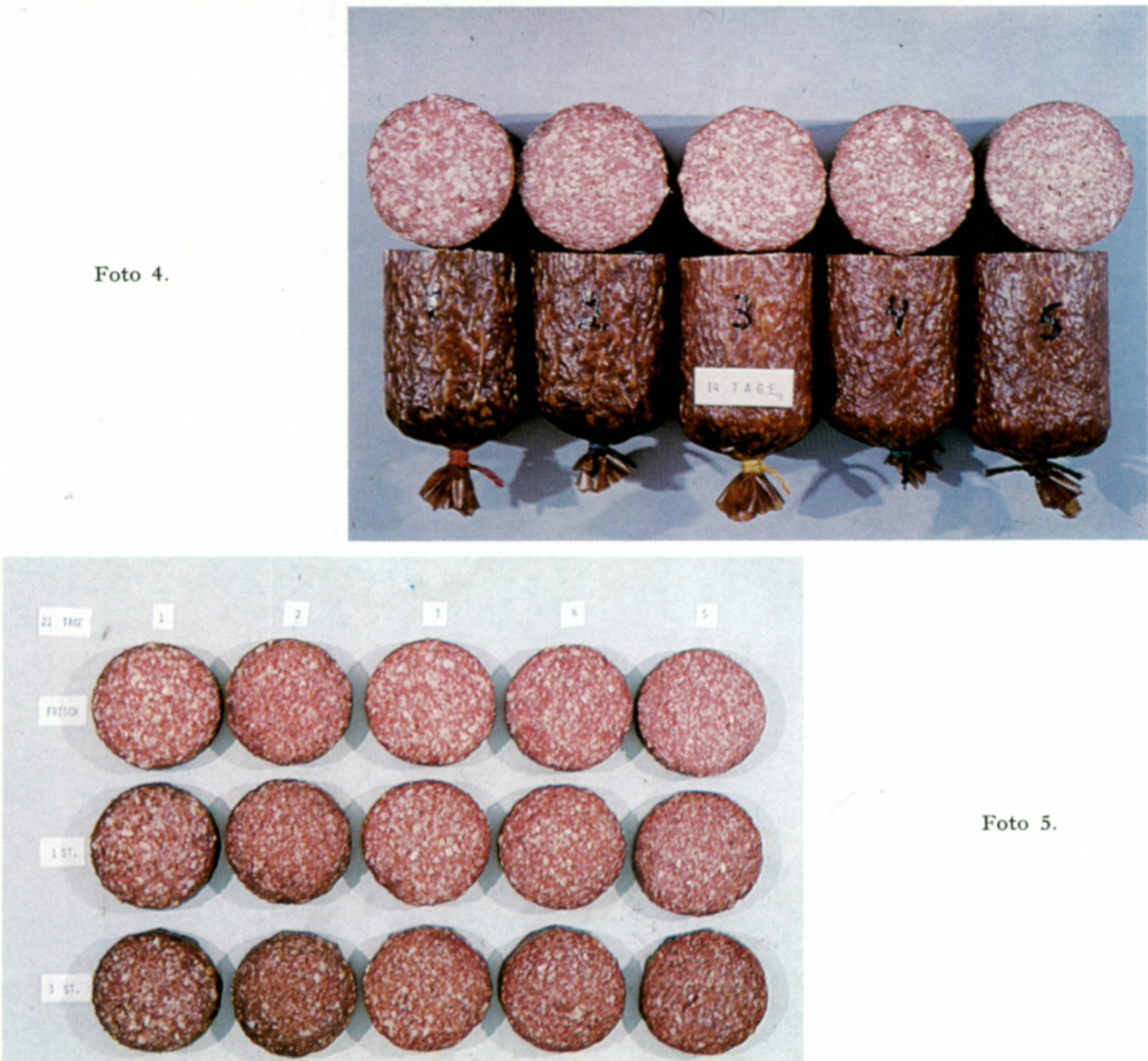

Foto 5. 